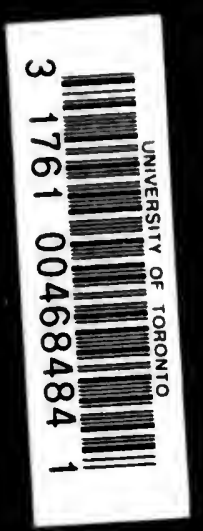




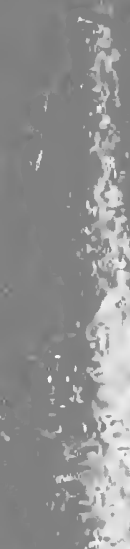

tas:

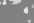

A

1.48
70

$-20 x$

$9 / 2$

is

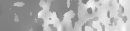

s)

$=4$. Wh

stris.
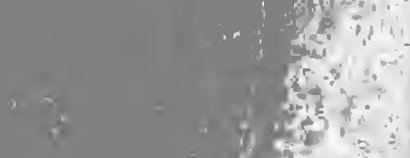

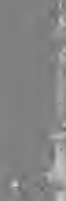

21
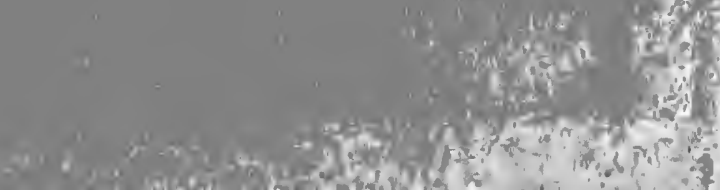


\section{THE COMBINATION OF OBSERVATIONS}




\section{CAMBRIDGE UNIVERSITY PRESS}

C. F. Clay, Manager

Ilondon: FETTER LANE, E.C.

ET̃inburgh : 100 , PRINCES STREET

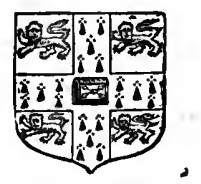

few gork: G. P. PUTNAM'S SONS

Bombag, Calcutta and fadras: MACMILLAN AND CO., LTD,

Toronto: J. M. DENT AND SONS, LTD. Tokno: THE MARUZEN-KABUSHIKI-KAISHA 
THE COMBINATION OF OBSERVATIONS

DAVID BRUNT, M.A. (Cantab.), B.Sc. (Wales)

Lecturer in Mathematics at the Monmouthshire Training College, Cacrleon, Mon.

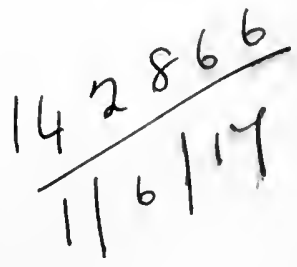

\section{Cambridge :}

at the University Press

I 917 
unknown quantity will be found in the first four Chapters. Many of the problems of the physicist involve one unknown only, and the first four Chapters contain all the theory that has to be considered in the discussion of such problems. The subject of Chapter VII, the adjustment of conditioned observations, has only been outlined very briefly. The fuller development of the subject, which forms the basis of the adjustment of triangulations, will be found in the works to which reference is made at the end of Chapter VII. The last four Chapters can only be regarded as mere introductions to the subjects discussed, but it was thought that their inclusion in a textbook on Least Squares would be an advantage.

I have to acknowledge my indebtedness to Mr F. J. M. Stratton, of Gonville and Caius College, Cambridge, to whose University lectures I owe most of my knowledge of the subjects discussed in this book, and upon whose notes I have drawn freely. I have also to thank Professor Eddington for many useful suggestions made while the book was in manuscript form, and for permission to extract from his lecture notes a number of interesting examples as well as some portions of the theoretical treatment.

I cannot express adequately my debt to the Cambridge University Press, for the extreme care shown in passing the book through the proof stage. Owing to my being abroad at the time, I was not able to devote as much time as was desirable to the reading of proofs, and but for the unfailing vigilance of the Press, many errors would have been allowed to pass into the text.

DAVID BRUNT.

Meteorological Section, R.E.

G. H. Q.

November 23, 1916. 


\title{
TABLE OF CON'TEN'TS
}

\author{
CHAPTER I \\ ERRORS OF OBSERVATIỌN
}

The nature of errors of observation

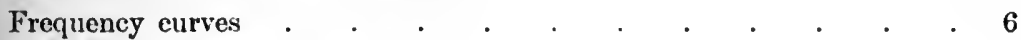

Accuracy of the arithmetic mean . . . . . . . . . . $\quad$. 9

\section{CHAP'TER II}

\section{THE LAW OF ERROR}

Hagen's proof of Gausss's error law . . . . . . . . . 11

Thomson and Tait's proof . . . . . . . . . . 14

Generalised form of Hagen's proof . . . . . . . . 15

Form of the error curve . . . . . . . . . . . 18

The arithmetic mean . . . . . . . . . . 21

Derivation of the normal law of errors from the principle of the arithmetic

mean . . . . . . . . . . . . . 22

Law of error of a linear function of two independent quantities. . 24

The median . . . . . . . . . . . . 27

Examples . . . . . . . . . . . . . . . 28

References. . . . . . . . . . . . . . . . . . 28

\section{CHAPTER III}

\section{THE CASE OF ONE UNKNOWN}

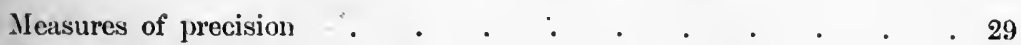

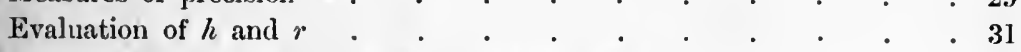

Comparison of a set of observations with theory. . . . . . 33

Evaluation of $\mu$. $\quad . \quad . \quad . \quad . \quad . \quad . \quad . \quad . \quad . \quad . \quad .33$

Probable error and mean square error of the arithmetic mean . . 36

Probable error of a linear function. . . . . . . . . 36

Probable error of the arithmetic mean . . . . . . . 37

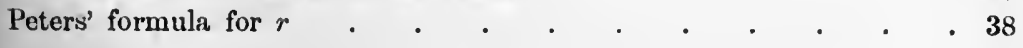


unknown quantity will be found in the first four Chapters. Many of the problems of the physicist involve one unknown only, and the first four Chapters contain all the theory that has to be considered in the discussion of such problems. The subject of Chapter VII, the adjustment of conditioned observations, has only been outlined very briefly. The fuller development of the subject, which forms the basis of the adjustment of triangulations, will be found in the works to which reference is made at the end of Chapter VII. The last four Chapters can only be regarded as mere introductions to the subjects discussed, but it was thought that their inclusion in a textbook on Least Squares would be an advantage.

I have to acknowledge my indebtedness to Mr F. J. M. Stratton, of Gonville and Caius College, Cambridge, to whose University lectures I owe most of my knowledge of the subjects discussed in this book, and upon whose notes I have drawn freely. I have also to thank Professor Eddington for many useful suggestions made while the book was in manuscript form, and for permission to extract from his lecture notes a number of interesting examples as well as some portions of the theoretical treatment.

I cannot express adequately my debt to the Cambridge University Press, for the extreme care shown in passing the book through the proof stage. Owing to my being abroad at the time, I was not able to devote as much time as was desirable to the reading of proofs, and but for the unfailing vigilance of the Press, many errors would have been allowed to pass into the text.

\section{DAVID BRUNT.}

Meteorological Section, R.E.

G. H. Q.

November 23, 1916. 


\title{
'TABLE OF CON'TEN'TS
}

\author{
CHAP'TER I \\ ERIRORS OF OBSERVATIỌN
}

The nature of errors of observation . . . . . . . . 1

Frequency curves . . . . . . . . . . . . . 6

Accuracy of the arithmetic mean . . . . . . . . . . 9

\section{CHAPTER II}

\section{THE LAW OF ERROR}

Hagen's proof of Gauss's error law . . . . . . . . . 11

Thomson and Tait's proof . . . . . . . . . . 14

Generalised form of Hagen's proof . . . . . . . . . 15

Form of the error curve. . . . . . . . . . . . 18

The arithmetic mean . . . . . . . . . . 21

Derivation of the normal law of errors from the principle of the arithmetic

mean . . . . . . . . . . . . . . 22

Law of error of a linear function of two independent quantities. . 24

The median . . . . . . . . . . . . 27

Examples . . . . . . . . . . . . . . . . . 28

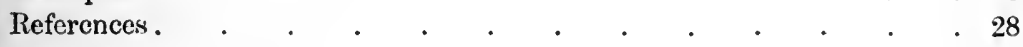

\section{CHAPTER III}

\section{THE CASE OF ONE UNKNOWN}

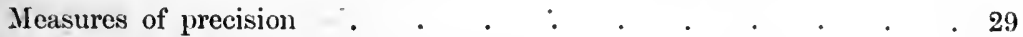

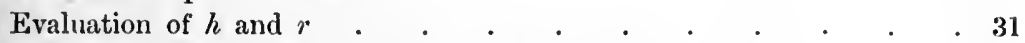

Comparison of a set of observations with theory . . . . . . 33

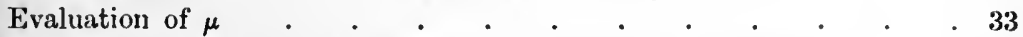

Probable error and mean square error of the arithmetic mean . $\quad 36$

Probable error of a linear function. . . . . . . . . . . 36

Probable error of the arithmetic mean . . . . . . . . 37

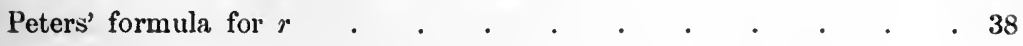


Examples of the aujustment of observations of one unknown $\quad$. $\quad 39$

Probable error of any function of a number of independent quantities . 48

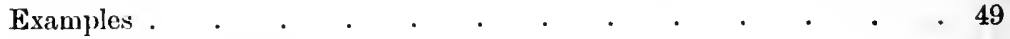

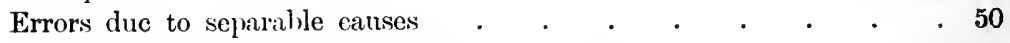

Probable error when a systematic error is present . . . . . 51

Correction of statistics for the effects of a known probable error of

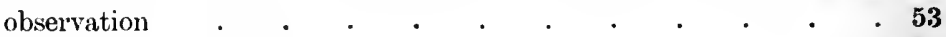

Precision of the probable error . . . . . . . . . . . 54

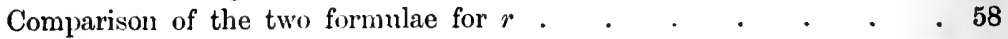

\section{CHAPTER IV}

\section{OBSERVATIONS OF DIFFEREN'T WEIGHT}

The weighting of observations . . . . . . . . . . . 60

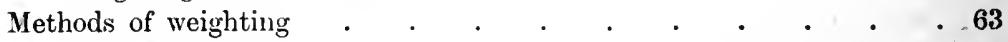

Alternative method of eraluating the precision of the weighted mean when weighting according to probable errors . . . . . ., 67

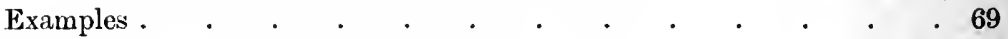

Miscellaneous Examples involving one unknown . . . . . 72

\section{CHAPTER V}

THE GENERAL PROBIEM OF THE ADJUSTMENT OF INDIRECT OBSERVATIONS INVOLVING SEVERAL UNKNOWN QUANTITIES

Statement of the problem . . . . . . . . . . 75

Formation of the normal equations . . . . . . . . 77

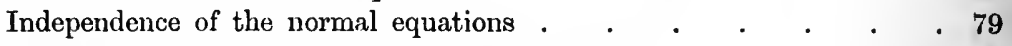

Checks on the formation of the normal equations . . . 80

Examples of the formation of normal equations . . . . . 82

Solution of the normal equations . $\quad . \quad$. $\quad . \quad . \quad . \quad . \quad$. 88

\section{CHAPTER VI}

EVALUATION OF THE MOST PROBABLE VALUES OF THE UNKNOWNS, THEIR WEIGHTS AND PROBABLE ERRORS

Gauss's method of substitution . . . . . . . . 90

Checks in computation. . . . . . . . . . . . 91

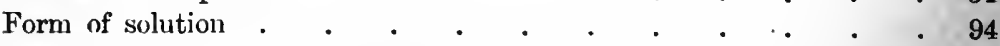

The Doolittle method of solution . . . . . . . . 96

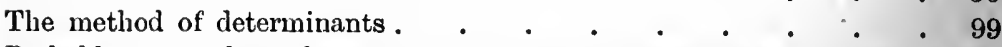

Probable error of an observational equation of unit weight.$=100$

Evaluation of probable errors of the unknowns . . . . . 103 
Evaluation of the weights of $x, y, z$, etc. . . . . . . 105

Examples. . . . . . . . . . . . . 107

Altemative method of finding the weights of the unknowns . . 108

Alternative proof of the rule for finding weights of the unknowns . 113

Alternative proof of the formula

$$
r=0.6745 \sqrt{\frac{[v v]}{n-m}} . \quad \cdot \quad \cdot \quad \cdot 115
$$

Probable error of any function of the unknowns. . . . . . 116

Normal place method in the formation of observational equations . 117

Testing the results of the least square solution for unusual errors, and

for systematic or constant errors. . . . . . . . . 118

Miscellaneous Examples. . . . . . . . . . . 120

\section{CHAPTER VII}

THE ADJUSTMENT OF CONDITIONED OBSERVATIONS

Statement of the problem . . . . . . . . . 124

Direct solution by substitution . . . . . . . . . . 125

Method of undeternined multipliers or correlates _ . . . 127

The precision of the unknowns . . . . . . . . 128

References . . . . . . . . . . . . . . . 128

\section{CHAPTER VIII}

THE REJECTION OF OBSERVATIONS • • . 129

\section{CHAPTER IX}

ALTERNATIVES TO THE NORMAL LAW OF ERRORS

Some statistical terms . . . . . . . . . . . . 133

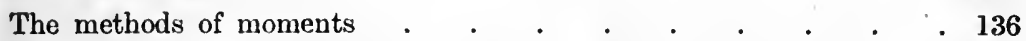

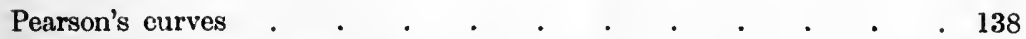

Integration of the differential equation . . . . . . . 141

Use of the series

$$
y=A_{0} \phi(x)+A_{3} \phi^{\prime \prime \prime}(x)+A_{4} \phi^{\mathrm{iv}}(x)+\text { etc. } \quad . \quad . \quad .145
$$

\section{CHAPTER $\mathrm{X}$}

\section{CORRELATION}

A simple example . . . . . . . . . . . . . . 148 The contingency table . . . . . . . . . . . 150 Adjustment of the line of regression by the method of least squares . 152 
Evaluation of $r$ for the material of figure 9 .

Calculation of $r$ from the contingency table.

The correlation ratio

Probable errors of $\sigma, r$, and $\eta$

Calculation of $\eta$ for the material of the table of page 151

The meaning of the correlation coefficient

\section{CHAPTER XI}

\section{HARMONIC ANALYSIS FROM THE STANDPOINT OF LEAST SQUARES}

To find the amplitude of a single periodic term of known period

General case involying more than one period . . . . . . . 173

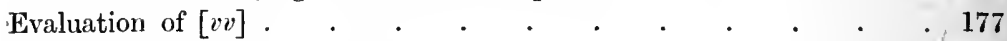

Case where $n$ terms are taken in the Fourier series . . . . 178

Fourier's Theorem . . . . . . . . . . . . . 179

Evaluation of the coefficients; $n$ a multiple of 4 . . . . 181

$" \quad " \quad n$ a multiple of 8 . . . . 185

Practical method of investigating periodicities . . . . . . 186

Examples . . . . . . . . . . . . . . 188

\section{CHAPTER XII}

\section{THE PERIODOGRAM}

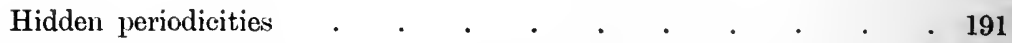

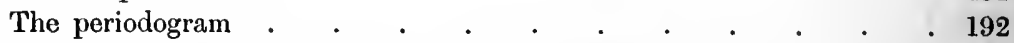

Form of the periodogram for one simple period . . . . . . 193

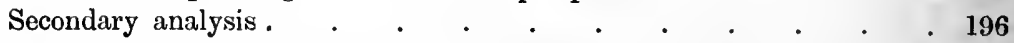

Fourier series for random distribution of observations . . . 199

Application to the periodogram . . . . . . . . 201

Schuster's investigations . . . . . . . . . . 202

Whittaker's variant of Schuster's method . . . . . . . 203

Use of the complete Fourier sequence. . . . . . . 204

The investigation of discontinuities . . . . . . . . $\quad .208$

An application of the Fourier sequence in periodogram work . $\quad 210$

Appendix I. List of References. $\quad . \quad$. $\quad . \quad$. $\quad$. 212

Appendix II. Table of $\Theta(t) \quad$ : . . . . . . . . 214

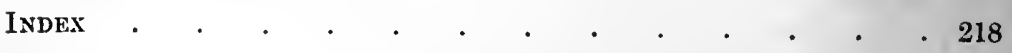




\section{CHAPTER I}

\section{ERRORS OF OBSERVATION}

1. THE first serious step in the advance of the sciences which are dependent upon measurements of any kind came with the introduction of instruments. Even the most primitive instruments yielded results far in advance of those obtained by mere estimation with the unaided human senses. With the course of time the development of new instruments has always been in the direction of greater refinement and accuracy. But even the inost refined instruments often fail to yield absolutely accurate results; for as a rule it is found that when a series of measurements of the same quantity is taken, the results do not show perfect agreement. The disagreement between individual observations in a series is attributed to errors of observation. Any observation which is of the nature of a measurement is affected by three factors, the instrument used, the external conditions at the time of observation, and the observer. Each of these factors may introduce errors into the observation. We shall consider these three factors in turn.

2. An instrument may be defined as a mechanical means of extending the ordinary human faculties, so as to yield measurements of greater refinement than are possible without its aid. The amount of water in a cup, the weight of a stone, the angular distance between two distant points, can be roughly determined by estimation, but measurements of far greater accuracy can be. obtained by pouring the water into a graduated vessel, weighing the stone on a balance, and measuring the angular distance between the distant points with a theodolite. The graduated meașure, the balance, and the theodolite are examples of instruments.

B. 0 . 
Just as the graduated vessel is liable to errors of graduation, so the most carefully made instrument is liable to errors of construction, and these errors affect the observations made with the instrument, producing what are known as "instrumental errors." The errors of construction of an instrument may be of the nature of errors of graduation of a scale, periodic errors in a screw, or maladjustment of the separate parts of the instrument. In order to obtain the best possible results, it is necessary to make a careful search for errors in construction, or in actual working, of the instrument, so that their effect may be eliminated. The errors may be of such a nature that it is possible to calculate their effect, and make an empirical correction. But, when possible, it is better to arrange the system of observations in such a way that the instrumental errors eliminate themselves. For example, if measurements have to be made by means of a scale graduated around a circle, it is possible to eliminate most of the errors due to eccentricity of the circle, as well as the greater part of the effect of periodic errors in the graduation of the scale, by placing an even number of verniers, say four or six, at equal distances around the circle*. When it is not possible to eliminate the instrumental errors by the adoption of a suitable method of observation, it may be necessary to carry out a special series of observations for the purpose of calculating empirical corrections for the measurements yielded by the instrument.

The use of refined instruments does not always diminish the difficulties of observation. "The more refined the methods employed, the more vague and elusive does the supposed magnitude become; the judgment flickers and wavers, until at last in a sort of despair some result is put down, not in the belief that it is exact, but with the feeling that it is the best we can make of the matter.$+ "$

3. The external conditions are such parts of the environment of the observer as affect either the observer or his instrument, and are beyond the observer's control; e.g. temperature, wind, or sunlight. If the external conditions be subject to violent change, it may be necessary to suspend work for a time. In some

* See Ball, Spherical Astronomy, p. 462.

† Lamb, Presidential Address to Brit. Assoc. 1904. 
cases it is possible to make corrections for the errors introduced by changing external conditions by means of an empirical law determined by an independent set of observations. Such corrections are never perfectly satisfactory, and the observer should avoid the necessity as far as possible, by observing only at times when the external conditions are steady.

4. The observer may have personal peculiarities which will affect all his observations. He may always measure an angle as larger (or smaller) than it really is, or he may always tend to note the passage of a star across the wires in the focal plane of an instrument a slight interval before (or after) the true time of transit. A careful and experienced observer appears to commit an error which is generally of the same sign, and approximately of the same magnitude, in a series of similar observations. Such an error is called the "personal equation" of the observer. It can be corrected by comparison with a fixed arbitrary standard. It should be noted, however, that only experienced observers have a well-defined personal equation. An inexperienced observer will commit errors of varying magnitude and sign, and even an experienced observer ceases to have a personal equation when not in his normal state. In order to obtain the best possible results, an observer should not continue to work when he is tired.

From these three sources there will arise errors of a more or less systematic nature, varying according to definite laws with the changing conditions of the observations. When the observed value has been corrected for these sources of error, we might expect the corrected observation to yield the true value of the quantity to be measured. But if a series of observations be made, and corrected in each case for the errors due to the three factors considered above, it will in general be found that the corrected measurements differ among themselves. These individual differences are ascribed to a fourth class of error, known as the accidental error.

5. Accidental errors are due to no known cause of systematic or constant error. They are irregular, and more or less unavoidable. The term "accidental" is not used here in its ordinary significance of "chance." Strictly speaking, an observation 
of any kind is affected by the state of the whole universe at the time of observation. But as an observer cannot take account of the whole universe and its changes of condition during the time occupied by his observations, he has to limit his attention to those operative causes which he regards as affecting his observations in a measurable degree; i.e. he limits his attention to the "essential conditions." If an observation could be repeated a number of times, and corrected in each case for changes in the essential conditions, the results of all the observations should be identical. But in practice it is found that the individual observations in a series differ among themselves. These differences may be ascribed to the fact that the so-called "essential conditions" do not include all the effective operative causes. There will be other operative causes of error, whose laws of action are unknown, or too complex to be investigated. These causes will introduce errors which will appear to the observer to be accidental.

We can now define accidental errors as errors whose causes and laws of action are unknown. The total accidental error in any individual measurement may be the sum of a number of small accidental errors arising from different causes. Among such errors would be accounted those arising from slight irregular changes in the external conditions, such as the vibration of the image of a distant object on account of air-currents, and the uncertainty of placing a cross-wire upon the image of a scale division; and also irregular changes in the personal equation of the observer. There will also be included in this class the remnants of instrumental errors, but if it should be possible to discover the law of action of any such error, it is thereby removed from the class of accidental errors to the class of systematic errors. Thus, when a distance is measured a large number of times with different parts of a scale, the errors of the scaledivision enter into the results in a more or less accidental manner. But if the scale errors be carefully investigated, their effect can be eliminated from the observed values. Carelessness in the handling of an instrument may introduce irregular instrumental errors which fall into the class of accidental errors.

It is thus seen that even when all the systematic errors traceable to the instrument, the external conditions, or the 
observer, have been corrected, no observation ean be regarded as perfect. It will miss perfection on account of the presence of accidental errors. The effect of the accidental errors will differ for different observations in the same series. It is thus impossible to attain certainty in the result of an observation. In practice a series of observations is made, in the hope that the discussion of the series will eliminate the effect of the accidental errors. The problem which iwe have to solve is that of deciding the best method of conducting this diseussion. Our problem may be briefly stated as follows. Given a series of observations, each of which has been made with all possible eare, and corrected for all known causes of error, how shall we determine the most probable values of the quantities to be determined? The material with which the theory deals is supposed to have been cleared of all constant and systematic errors, and to be subject only to accidental errors, whose laws of action are unknown. The values of the observations, thus corrected, so as to be subject only to accidental errors will in future be referred to as the "observed values."

In what follows, accidental errors will be regarded as obeying the following laws:

(i) A large number of very small accidental errors are present in any observation.

(ii) A positive error and an equal negative error are equally probable.

(iii) The total error cannot exceed a certain reasonably small amount.

(iv) The probability of a small error is greater than the probability of a large error.

As we shall frequently have to refer to constant and systematic errors in the sequel, it will be well to have a clear conception of the meaning of these terms. A constant error is one which has the same effect upon all the observations in a series. It has the same magnitude and sign in all the observations. A systematic error is one whose sign and magnitude bear a fixed relation to one or more of the conditions of observation. It should be noted that neither of these types of error fulfils the laws of accidental errors given above. 


\section{Frequency Curves.}

Before proceeding further with the theoretical discussion of errors, we shall consider briefly the general nature of the materia] with which the theory has to deal. The table given below shows the results of a series of observations extracted from the account of the preliminary experiments on photographic transits at the Observatory of Tokio. The first and third columns give the actual observations, while the second and fourth columns give the deviations of the individual observations from the arithmetic mean $(4.986)$ in units of the third decimal place*.

$\begin{array}{rrrr}4 \cdot 974 & -12 & 4 \cdot 978 & -8 \\ 82 & -4 & 93 & 7 \\ 78 & -8 & 88 & 2 \\ 89 & 3 & 83 & -3 \\ 93 & 7 & 5 \cdot 001 & 15 \\ 79 & -7 & 5 \cdot 015 & 29 \\ 84 & -2 & 4 \cdot 993 & 7 \\ 87 & 1 & 91 & 5 \\ 5 \cdot 001 & 15 & 74 & -12 \\ 4 \cdot 997 & 9 & 71 & -15 \\ 86 & 0 & 67 & -19 \\ 78 & -8 & 91 & 5 \\ 83 & -3 & 88 & 2 \\ 83 & -3 & 84 & -2 \\ 90 & 4 & 72 & -14 \\ 91 & 5 & 72 & -14\end{array}$

The deviations from the arithmetic mean vary from -19 to +29 .

These deviations (or the actual observations) may be represented graphically as follows (fig. 1). Let the deviations be measured along the horizontal axis, and the number of observations along the vertical axis. Divide the total range of deviation into a number of intervals, say 0 to $\pm 5, \pm 5$ to \pm 10 , etc. For each observation put a dot along the ordinate through the middle point of the interval within which it falls, successive dots on the same ordinate being placed at unit distance apart. The height of the last dot on any ordinate gives the number of observations

\footnotetext{
* These deviations are called the residuals.
} 
which fall within the corresponding deviation interval. If the tops of all the ordinates be joined, the resulting broken line represents the frequency of the different mensurements, and is called the frequency curve (see figure 1).

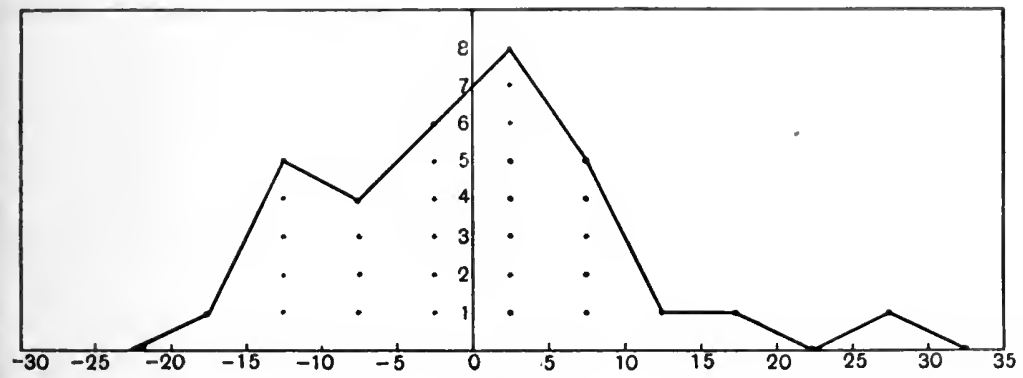

Fig. 1.

Curves obtained by this method, for a moderate number of observations, will generally show one or more peaks, some horizontal portions, and may at some points lie along the horizontal axis. Similar curves will be found in figures 5,6, 7, and 8. An alternative method of completing the diagram, instead of joining the tops of successive ordinates, is to draw a rectangle whose height is equal to an ordinate, and whose breadth extends over the class-interval, as shown in figure 2. Such a diagram is called a histogram, or a frequency-polygon.

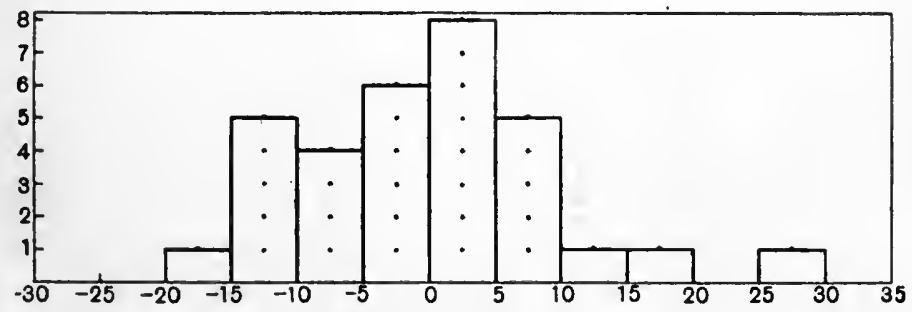

Fig. 2.

Histogram of material shown in figure 1.

If we compare the frequency eurves obtained by plotting the repetitions of an observation, using at first a small number of 
repetitions, and then larger and larger numbers, we find that for a small number of observations the curve shows peaks and valleys and horizontal portions, and changes its form considerably when the number of observations is changed. But as the number of observations becomes larger, the frequency curve tends to approach a fixed form, having in general a single maximum ordinate, while the curve slopes down towards the axis on each side of the maximum. It then becomes convenient to draw a smooth curve throngh the tops of the ordinates. As the number of observations is still further increased, the successive frequency curves become more and more similar in form, until, when the number of observations is very great, there is no appreciable difference in the form of successive frequency curves. The final form of the curve is that which represents the frequency distribution of an indefinitely large number of observations. This final curve is called the "curve of presumptive errors."

In practice, it is never possible to repeat an observation an indefinitely large number of times, and we have to content ourselves with regarding the frequency curve obtained from a finite number of observations as yielding a reasonably good approximation to the curve of presumptive errors.

The study of a large number of curves of presumptive errors shows a decided similarity in their form, and a strong tendency to approach a typical form distinguished by symmetry about the maximum ordinate. The approach to the typical form is so striking that it is a matter of extreme importance to investigate the possible analytical form of the curve. In the next chapter, starting from certain hypotheses, we shall deduce an analytical formula to represent the typical curve. The purpose of the formula is to express the proportion of the total number of observations whose errors shall lie between any assigned limits, say $\Delta$ and $\Delta+d \Delta$; in other words, it will express the probability that the error of a single observation shall lie between $\Delta$ and $\Delta+d \Delta$. The use of the words "frequency" and "probability" to denote the same thing is common to writers on this subject. A moment's consideration of the definition of probability will indicate a justification of this custom.

"If, on taking a large number $N$ out of a series of cases in 
which an event $A$ is in question, $A$ happens on $p N$ occasions, the probability of the event $A$ is said to be $p . "$

The quantity $p$, so defined, is also the relative frequency of the event $A$.

The law which is commonly held to represent the typical curve of errors is Gauss's Error Law, or the Law of Least Squares, according to which the probability that an observation should have an error between $\Delta$ and $\Delta+d \Delta$ is $\frac{h}{\sqrt{ } \pi} e^{-h^{2} \Delta^{2}} d \Delta$, where $h$ is a constant depending on the closeness of the agreement between the observations in the series. This expression will be derived in the next chapter, and its validity will be tested by its application to the adjustment of a number of series of observations. Meanwhile it may be noted that a number of useful results can be derived from the assumption that positive and negative errors are equally probable. Thus the accuracy of the arithmetic mean can be investigated without reference to the actual form of the Law of Errors.

\section{The Accuracy of the Arithmetic Mean.}

If $m_{1}, m_{2}, \ldots, m_{n}$ be $n$ determinations of a single unknown quantity, the arithmetic mean is given by

$$
a=\frac{1}{n}\left(m_{1}+m_{2}+\ldots+m_{n}\right) \text {. }
$$

If $y_{1}, y_{2}, \ldots, y_{n}$ be the errors of the individual determinations, and $x$ the error of the arithmetic mean, then

$$
x=\frac{1}{n}\left(y_{1}+y_{2}+\ldots+y_{n}\right) .
$$

Squaring each side of this equation, we find

$$
x^{2}=\frac{1}{n^{2}}\left(y_{1}^{2}+y_{2}^{2}+\ldots+y_{n}^{2}\right)+\frac{2}{n^{2}}\left(y_{1} y_{2}+y_{1} y_{3}+y_{2} y_{3}+\text { etc. }\right) .
$$

The mean value of $x^{2}$, say $\bar{x}^{2}$, is equal to the mean value of the right-hand side of this equation. Since positive and negative errors are equally likely to occur, and all the $y$ 's are independent, then in a large number of trials the mean value of $y_{r} y_{s}$ will be zero. And since all the $n$ observations considered are supposed to 
be carried out in precisely the same manner. we may expect the mean values of $y_{1}^{2}, y_{2}^{2}, \ldots, y_{n}{ }^{2}$ to be equal. If the mean value of each of the squared terms $y_{1}^{2}, y_{2}^{2}$, etc., be $\mu^{2}$, then

$$
\bar{x}^{2}=\frac{1}{n^{2}}\left(n \mu^{2}\right)=\frac{\mu^{2}}{n}
$$

and

$$
\bar{x}=\frac{\mu}{\sqrt{n}} .
$$

Hence it follows that the accuracy of the arithmetic mean is $\sqrt{n}$ times the accuracy of a single observation; a result of fundamental importance in the theory of errors. 


\section{CHAPTER II}

\section{THE LAW OF ERROR}

\section{Hagen's Proof of Gauss's Error Law*.}

Hagen based his proof on the assumption that an accidental error consists of the algebraic sum of a very large number of infinitesimal errors, all of equal magnitude, and as likely to be positive as negative. It has already been suggested that the accidental error occurring in any one observation may be composed of a number of errors due to slight changes in the external conditions, remnants of instrumental errors, or irregularities in the individual peculiarities of the observer. Each of these components may in turn be regarded as due to a large number of elementary causes. And so Hagen's hypothesis is in no way $a=$ violation of our knowledge of the nature of accidental errors.

Let the total number of elementary errors be $n$, where $n$ is a number to which we can assign no limit. If the magnitude of each of the elemental errors be $\epsilon$, then it will be possible for an error as great as $n \epsilon$ to occur, in the extreme case where all the small errors occur with the same sign. An error $(n-2 m) \epsilon$ will occur when $n-m$ of the elemental errors occur with a positive sign, and the remaining $m$ with a negative sign. The number of ways in which this can happen is simply the number of ways in which we can select $m$ out of the $n$ errors. The selected $m$ errors will have one sign, and the remaining $n-m$ will have the opposite sign. The selection can be made in

$$
\frac{n !}{n-m ! m !} \text { ways. }
$$

* Hagen, Gründzuge der Wahrscheinlichkeitsrechnung (Berlin, 1837). 
Let $x-\epsilon$ be the error due to $n-m$ errors $+\epsilon$, and $m$ errors $-\epsilon$. Then

$$
\begin{aligned}
& x-\epsilon=(n-2 m) \epsilon \\
& x=(n-2 m+1) \epsilon .
\end{aligned}
$$

or

If $f(x-\epsilon)$ be the frequency of the error $x-\epsilon$,

$$
f(x-\epsilon)=\frac{n !}{n-m ! m !} .
$$

An error $x+\epsilon$, or $(n-2 m+2) \epsilon$, will be formed by $n-m+1$ elemental errors $+\epsilon$, and $m-1$ errors $-\epsilon$. Hence

$$
f(x+\epsilon)=\frac{n !}{n-m+1 ! m-1 !} \text {. }
$$

It follows that

$$
\frac{f(x+\epsilon)}{f(x-\epsilon)}=\frac{m}{n-\dot{m}+1} .
$$

Since the elemental errors $\epsilon$ are supposed to be infinitesimally small in comparison with the finite composite errors of actual observations, the latter may be assumed to vary continuously from $-n \epsilon$ to $+n \epsilon$, the extreme error $\pm n \epsilon$, whose relative frequency is very small, being assumed to be infinite. If we define $\phi(x) d x$ as the proportion of errors between $x-\frac{1}{2} d x$ and $x+\frac{1}{2} d x, \phi(x)$ may be regarded as a continuous function. But the function $f(x)$ gives the frequency of the errors $x$, where all the possible values of $x$ are separated by intervals of $2 \epsilon$. We may bring the function $\phi(x)$ and $f(x)$ into line by regarding $f(x)$ as the frequency of all errors between $x-\epsilon$ and $x+\epsilon$. We then have the equation

$$
f(x)=C \phi(x) .2 \epsilon \text {. }
$$

It follows that

$$
\begin{gathered}
\frac{\phi(x+\epsilon)}{\phi(x-\epsilon)}=\frac{f(x+\epsilon)}{f(x-\epsilon)}=\frac{m}{n-m+1}, \\
\frac{\phi(x+\epsilon)-\phi(x-\epsilon)}{\phi(x+\epsilon)+\phi(x-\epsilon)}=-\frac{(n-2 m+1)}{n+1} .
\end{gathered}
$$

Neglecting squares of $\epsilon$, we may write

$$
\begin{aligned}
& \phi(x+\epsilon)=\phi(x)+\epsilon \frac{d \phi}{d x}, \\
& \phi(x-\epsilon)=\phi(x)-\epsilon \frac{d \phi}{d x} .
\end{aligned}
$$

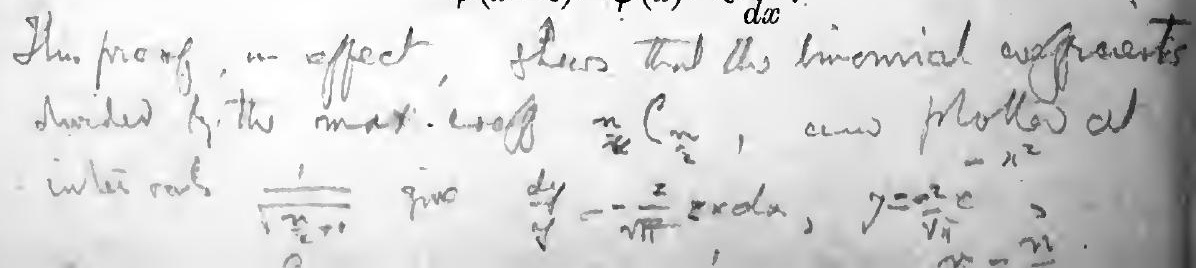


Substituting these values in the above equation, we find

$$
\begin{aligned}
& \frac{2 \epsilon \frac{d \phi}{d x}}{2 \phi(x)}=-\frac{n-2 m+1}{n+1}=-\frac{x}{(n+1) \epsilon}, \\
& \frac{1}{\phi} \cdot \frac{d \phi}{d x}=-\frac{x}{(n+1) \epsilon^{2}} .
\end{aligned}
$$

Integrating this equation, we find

where

$$
\begin{aligned}
\log \phi(x) & =-\frac{x^{2}}{2(n+1) \epsilon^{2}}+\text { const. } \\
& =-h^{2} x^{2}+\text { const. } \\
h^{2} & =\frac{1}{2(n+1) \epsilon^{2}}, \\
\phi(x) & =A e^{-h^{2} x^{2}} .
\end{aligned}
$$

The constant $h^{2}$ depends upon the nature of the errors entering into the observations. The value of the constant $A$ is easily derived. $\phi(x) \cdot d x$ gives the proportion of errors between the limits $x-\frac{1}{2} d x$ and $x+\frac{1}{2} d x$, or, practically, between the limits $x$ and $x+d x$. Since all the possible errors must lie between $-\infty$ and $+\infty$, it follows that

$$
\begin{aligned}
\int_{-\infty}^{+\infty} \phi(x) d x & =1=A \int_{-\infty}^{+\infty} e^{-h^{2} x^{2}} d x \\
& =2 A \int_{0}^{\infty} e^{-h^{2} x^{2}} d x \\
& =2 A \times \frac{\sqrt{\pi}}{2 h} . \\
\therefore \quad A & =\frac{h}{\sqrt{\pi}} .
\end{aligned}
$$

Finally, we may write

$$
\phi(x)=\frac{h}{\sqrt{ } \pi} e^{-h^{2} x^{2}}=d x .
$$

This is the functional form of Gauss's Error Law, or, as it is sometimes called, the Normal Error Law. Its interpretation is that the relative number of observations in a series, whose errors lie within the limits $x$ and $x+d x$, is

$$
\frac{h}{\sqrt{ } \pi} e^{-h^{2} x^{2}} d x \text {. }
$$


In a long series of observations, $n$ in number, the number of observations whose errors lie between $x$ and $x+d x$ is

$$
\frac{n h}{\sqrt{\pi}} e^{-h^{2} x^{2}} d x
$$

\section{Thomson and Tait's Proof of the Error Law.}

Consider the distribution of shots fired at a target. Let axes of coordinates be drawn through the centre of the target, the $x$-axis horizontal, the $y$-axis vertical. Let $P,(x, y)$, be the mark of one shot on the target. Then $x$ and $y$ are components of the error of placing the point $P$. Since the shot is as likely to go to the right as to the left of the centre of the target, the probability of an error between $x$ and $x+d x$ in the $x$-coordinate is of the form $\phi\left(x^{2}\right)$. dx. Similarly the probability of an error between $y$ and $y+d y$ in the other coordinate is of the form $\phi^{\prime}\left(y^{2}\right) \cdot d y$. We shall assume that the functions $\phi$ and $\phi^{\prime}$ are of the same form. This is equivalent to assuming that a large number of shots fired at the target would be distributed indiscriminately about the centre, showing no special symmetry of distribution about the horizontal and vertical axes, as opposed to any other axis through the centre of the target.

Then the probability that the point $P$ should have coordinates lying between $x$ and $x+d x$, and between $y$ and $y+d y$, respectively, will be

$$
\phi\left(x^{2}\right) \cdot \phi\left(y^{2}\right) \cdot d x \cdot d y .
$$

In other words, the probability that the point $P$ should lie within a given small region of area $d A$ is

$$
\phi\left(x^{2}\right) \cdot \phi\left(y^{2}\right) \cdot d A,=
$$

$x$ and $y$ being the coordinates of any point within the area $d A$.

But if another pair of axes $O x^{\prime}, O y^{\prime}$ be drawn through the centre of the target, so that $O x^{\prime}$ passes through the area $d A$, the probability that a single shot should be placed within the area $d A$ can also be expressed by

or

$$
\begin{gathered}
\phi\left(x^{\prime 2}\right): \phi\left(y^{\prime 2}\right) d A \\
\phi\left(x^{2}+y^{2}\right) \cdot \phi(0) d A . \\
\therefore \quad \phi\left(x^{2}\right) \cdot \phi\left(y^{2}\right)=\phi\left(x^{2}+y^{2}\right) \cdot \phi(0) .
\end{gathered}
$$


This is a functional equation whose solution is

$$
\phi\left(x^{2}\right)=A e^{k x^{2}} .
$$

Since small errors are more probable than large errors, $\phi\left(x^{2}\right)$ must decrease as $x$ increases, and $k$ inust therefore be negative. Putting $k=-h^{2}$, we can show as before that

$$
A=\frac{h}{\sqrt{\pi}} \text {. }
$$

Finally we may write

$$
\phi\left(x^{2}\right)=\frac{h}{\sqrt{ } \pi} e^{-h^{2} x^{2}}
$$

which agrees with the form of the error law derived above $(\$ 8)$.

\section{A Generalised Form of Hagen's Proof*.}

Suppose that the error of observation is made up of a large number of independent elementary errors, and that the probability that any one of these elementary errors shall lie between $\epsilon$ and $\epsilon+d \epsilon$ is $g(\epsilon) d \epsilon$. We shall assume that positive and negative errors are equally likely, so that $g(\epsilon)$ is an even function, but otherwise $g(\epsilon)$ may be regarded as being quite arbitrary. Then

$$
\begin{aligned}
& \int_{-\infty}^{+\infty} g(\epsilon) d \epsilon=1, \\
& \int_{-\infty}^{+\infty} \epsilon g(\epsilon) d \epsilon=0 .
\end{aligned}
$$

Further let

$$
\int_{-\infty}^{+\infty} \epsilon^{2} g(\epsilon) d \epsilon=k^{2}
$$

The quantity $k$ so defined may be called the mean square elementary error.

Let

$$
\sqrt{k^{2}}=f(n, x) \cdot d x=\overline{k^{2}} \cdot d x
$$

be the probability that the resultant error due to $n$ of the elementary errors lies between $x$ and $x+d x$. Then we have

$$
f(n+1, x)=\int_{-\infty}^{+\infty} f(n, x-\epsilon) \cdot g(\epsilon) d \epsilon .
$$

This equation expresses the fact that the probability that $n+1$ errors add up to $x$, is made up of the probability that the first

* The above proof is due to Professor Eddington. 
$n$ errors add up to $x-\epsilon$, multiplied by the probability that the remaining error is $\epsilon$, summed for all possible values of $\epsilon$.

Expand both sides of the equation by Taylor's Theorem*.

$$
\begin{aligned}
f(n, x) & +\frac{\partial}{\partial n} \cdot f(n, x)+\ldots \\
& =\int_{-\infty}^{+\infty}\left\{f(n, x)-\epsilon \frac{\partial}{\partial x} f(n, x)+\frac{1}{2} \epsilon^{2} \frac{\partial^{2}}{\partial x^{2}} f(n, x)-\text { etc. }\right\} g(\epsilon) d \epsilon \\
& =f(n, x)+\frac{1}{2} k^{2} \frac{\partial^{2}}{\partial x^{2}} f(n, x)+\ldots \text { by }(1) .
\end{aligned}
$$

Write $n k^{2}=t$. Then $\sqrt{t}$ is the mean square error of the observations, and remains finite when $n$ is taken infinitely great, and $k^{2}$ accordingly infinitely small.

We then have

$$
f+k^{2} \frac{\partial f}{\partial t}+\ldots=f+\frac{1}{2} k^{2} \frac{\partial^{2} f}{\partial x^{2}}+\ldots
$$

the omitted terms being of the order of $k^{\star}$, when $k$ is small. Taking now a very large number of very small elementary errors, so that $k^{4}$ is negligible in comparison with $k^{2}$, this gives

$$
\frac{\partial f}{\partial t}=\frac{1}{2} \frac{\partial^{2} f}{\partial x^{2}}
$$

It is easily verified that, a solution of this equation is

$$
f=C t^{-\frac{1}{2}} e^{-\frac{x^{2}}{2 t}}
$$

It remains to show that this is the solution applicable to our problem. The differential equation (2) is the same as that which determines the conduction of heat along a bar. Now when the mean square error of an observation is zero, the probability of an error $x$ vanishes except for $x=0$; but unit probability is then concentrated into an infinitesimal range at $x=0$. The corresponding condition in the heat problem is that initially $(t=0)$ the bar is everywhere at zero temperature except that a unit quantity of heat is concentrated in it at the point $x=0$. Also, whatever be the mean square error, $f$ must vanish at $x=+\infty$ and $x=-\infty$; in the heat problem this means that the temperature at the two infinitely distant ends is zero. Now it is known from the theory

* Difficulties as to the possible divergence of the Taylor expansion for large values of $\epsilon$ may be avoided by introducing the additional assumption that $g(\epsilon)=0$ for values of $\epsilon$ beyond certain limits. 
of the conduction of heat that these two conditions-the initial condition and the end condition-are sufficient to determine uniquely a solution of the equation. Hence if we can obtain a solution for $f$ satisfying (1) $f=0$ when $t=0$, for all values of $x$ except $x=0$, and (2) $f=0$ when $x=+\infty$, and $x=-\infty$, for all values of $t$, this will be the only possible solution for the error law. It is easily seen that the solution (3) does satisfy these conditions.

The constant $C$ must be chosen so that

and writing

$$
\int_{-\infty}^{+\infty} f \cdot d x=1
$$

$$
h^{2}=\frac{1}{2 t}
$$

we obtain the expression in the usual form,

$$
f=\frac{h}{\sqrt{\pi}} e^{-h^{2} x^{3}}
$$

11. The proofs of the Normal Error Law given above are based on certain definite hypotheses concerning the nature of accidental errors. It has been shown that, if the accidental errors to which a series of observations is liable satisfy these hypotheses, the errors of observation will be distributed according to the normal law. The final justification of the use of Gauss's Error Curve rests upon the fact that it works well in practice, and yields curves which in very many cases agree very closely with the observed frequency curves. The normal law is to be regarded as proved by experiment, and explained by Hagen's hypothesis. When the curve of frequency of the actual errors is not of the form of the normal curve, we may safely conclude that the nature of the accidental errors concerned is not in accordance with Hagen's hypothesis. de/mulue.

The normal curve has applications in a region where deviations from a mean value are considered, though these deviations are not, properly speaking, of the same nature as accidental errors of observation; e.g., it is frequently applied in biological questions (vide Ex. 3, p. 42). The use of the normal curve in such cases is justified only when the differences between individual cases are produced by causes whose mode of action is in accordance with Hagen's hypothesis.

B. 0 .

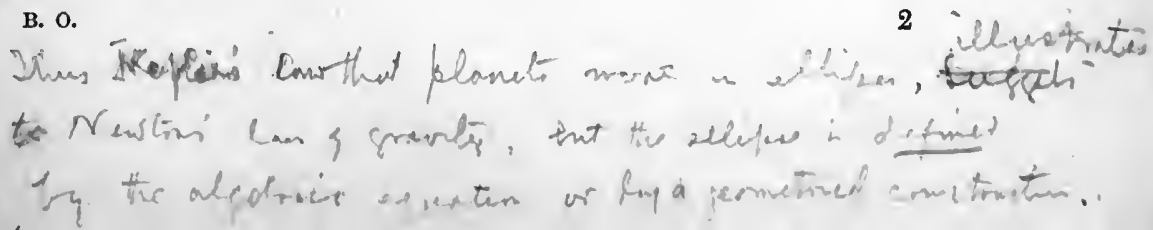




\section{The Form of the Frror Curve.}

The equation of the Error Curve is

$$
y=\frac{h}{\sqrt{\pi}} e^{-h^{2} x^{2}}
$$

The curve is symmetrical about the axis of $y$. The maximum ordinate occurs at $x=0$, and has the value $\frac{h}{\sqrt{ } \pi}$. The curve can easily be constructed by the use of tables of logarithms.

Differentiating this equation twice we obtain

$$
\frac{d^{2} y}{d x^{2}}=\frac{2 h^{3}}{\sqrt{\pi}}\left(2 h^{2} x^{2}-1\right) e^{-h^{2} x^{2}}
$$

showing that the curve has points of inflexion at

$$
x= \pm \frac{1}{\sqrt{2} h} .
$$

The form of the curve is shown in figure 3.

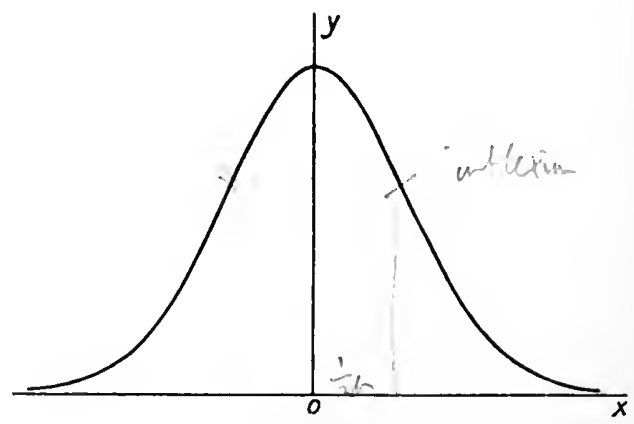

Fig. 3.

Gauss's Error Curve.

The probability that the error of an observation shall lie between $a$ and $b$ is

$$
\frac{h}{\sqrt{\pi}} \int_{a}^{b} e^{-h^{2} x^{2}} d x, \text { or } \frac{1}{\sqrt{\pi}} \int_{h a}^{h b} e^{-t^{2}} d t
$$

The value of this integral can be derived from the table below. The table gives the value of $\Theta(t)$, which is equal to

$$
\frac{2}{\sqrt{\pi}} \int_{0}^{p x} e^{-t^{2}} d t
$$


for a series of values of $x$ from 0 to 5.0, $\rho$ being a constant whose value is 0.47696 , the reason for the use of which will appear later.

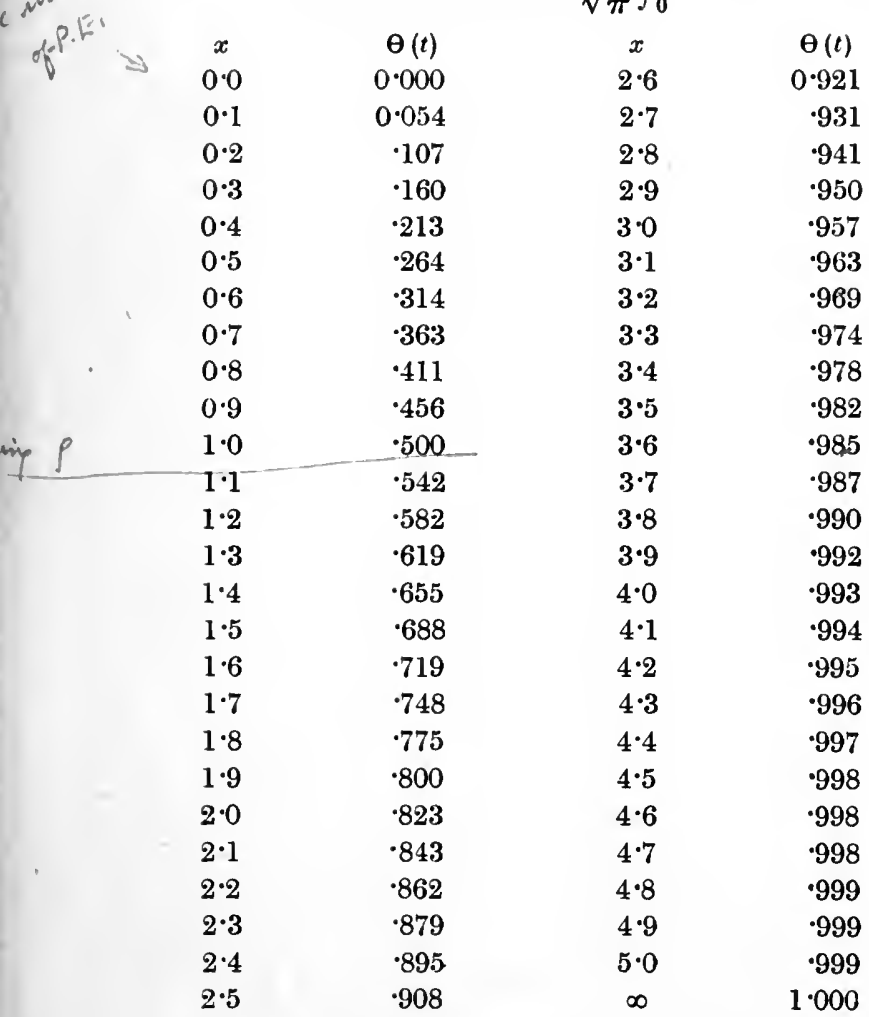

A. table of values of $\frac{2}{\sqrt{\pi}} \int_{0}^{t} e^{-t^{2}} d t$ is given in Appendix II.

For values of $x$, the integral $\int_{0}^{x} e^{-x^{2}} d x$ may be obtained by expanding $e^{-x^{2}}$.

$$
e^{-x^{2}}=1-x^{2}+\frac{1}{2} x^{4}-\frac{1}{1.2 .3} x^{6}+\text { etc. }
$$

This series is uniformly convergent, and may therefore be integrated term by term.

$$
\int_{0}^{x} e^{-x^{2}} d x=x-\frac{x^{3}}{1 ! 3}+\frac{x^{5}}{2 ! 5}-\frac{x^{7}}{3 ! 7}+\text { etc. }
$$


When $x$ is not small the above series converges very slowly, and it is then better to use another formula obtained by integrating by parts.

$$
\begin{aligned}
\int e^{-x^{2}} d x & =-\frac{1}{2 x} e^{-x^{2}}-\frac{1}{2} \int \frac{1}{x^{2}} e^{-x^{2}} d x \\
& =-\frac{1}{2 x} e^{-x^{2}}+\frac{1}{2 x^{3}} e^{-x^{2}}+\frac{1.3}{2^{2}} \int \frac{1}{x^{4}} e^{-x^{2}} d x .
\end{aligned}
$$

Continuing the process we find

$$
\int_{x}^{\infty} e^{-x^{2}} d x=\frac{e^{-x^{2}}}{2 x}\left\{1-\frac{1}{2 x^{2}}+\frac{1.3}{\left(2 x^{2}\right)^{2}}-\frac{1.3 .5}{\left(2 x^{2}\right)^{3}}+\text { etc. }\right\},
$$

and since

$$
\int_{0}^{x} e^{-x^{2}} d x=\int_{0}^{\infty} e^{-x^{2}} d x-\int_{x}^{\infty} e^{-x^{2}} d x=\frac{\sqrt{\pi}}{2}-\int_{x}^{\infty} e^{-x^{2}} d x,
$$

we can apply the second series to evaluate $\int_{0}^{x} e^{-x^{2}} d x$.

When $x$ is large a still better method is to use a series due to Schlomilch*.

$$
\begin{aligned}
\int_{x}^{\infty} e^{-x^{2}} d x=\frac{e^{-x^{2}}}{\sqrt{2} x}\left\{1-\frac{1}{2\left(x^{2}+1\right)}+\frac{1}{2^{2}\left(x^{2}+1\right)\left(x^{2}+2\right)}\right. & \\
& -\frac{5}{2^{3}\left(x^{2}+1\right)\left(x^{2}+2\right)\left(x^{2}+3\right)}+\frac{9}{2^{4}\left(x^{2}+1\right)\left(x^{2}+2\right)\left(x^{2}+3\right)\left(x^{2}+4\right)} \\
& \left.-\frac{129}{2^{5}\left(x^{2}+1\right)\left(x^{2}+2\right)\left(x^{2}+3\right)\left(x^{2}+4\right)\left(x^{2}+5\right)}+\ldots \text { etc. }\right\} .
\end{aligned}
$$

This series converges very rapidly for large values of $x$.

The error curve drawn above, represented by the function

$$
y=\frac{h}{\sqrt{\pi}} e^{-h^{2} x^{2}}
$$

extends to infinity along the axis of $x$, in both directions, having the axis of $x$ as an asymptote. In a system of errors accurately represented by this curve, the errors are continuous, and extend from $-\infty$ to $+\infty$. In actual observations the errors are never greater than a reasonably small finite limit, and the curve which would accurately represent the system of errors should meet the $x$-axis at a finite distance from the origin. But as the normal

* Kompendium der höhern Analysis, Bd. II, p. 266. 
curve of errors shown in figure 3 rapidly approaches the axis of $x$, so that its ordinates become infinitesimally small at a short distance from the origin, no considerable error is introduced by regarding the curve of actual errors as approaching the axis of $x$ asymptotically, and so regarding the limits of possible errors of measurement as being extended to $\pm \infty$.

\section{The Arithmetic Mean.}

If $x_{1}, x_{2}, \ldots, x_{n}$ be $n$ observed values of a quantity $x$, the errors of the separate observations are $x_{1}-x, x_{2}-x$, etc. The probability of making this system of errors is proportional to

$$
e^{-h^{2} \Sigma\left(x_{r}-x\right)^{2}}
$$

The value of $x$ must be so chosen that this probability shall be a maximum, or so that $\Sigma\left(x_{r}-x\right)^{2}$ shall be a minimum. Differentiating the last expression we obtain an equation for $x$,

$$
\Sigma\left(x_{r}-x\right)=0 \text {, or } \Sigma x_{r}-n x=0 \text {. }
$$

Hence

$$
x=\frac{1}{n} \Sigma x_{r}=\frac{1}{n}\left(x_{1}+x_{2}+\ldots+x_{n}\right) \text {. }
$$

The most probable value of the unknown is therefore the arithmetic mean of the observed values.

This result may be obtained without the aid of the differential calculus. If $\bar{x}$ be the arithmetic mean of $x_{1}, x_{2}, \ldots, x_{n}$, and $x$ any other value of the unknown,

$$
\begin{aligned}
\Sigma\left(x_{r}-x\right)^{2}-\Sigma\left(x_{r}-\bar{x}\right)^{2} & =n\left(x^{2}-\bar{x}^{2}\right)-2(x-\bar{x}) \Sigma x_{r} \\
& =n\left(x^{2}-\bar{x}^{2}\right)-2 n \bar{x}(x-\bar{x}) \\
& =n(x-\bar{x})^{2} . \\
\therefore \Sigma\left(x_{r}-x\right)^{2}=\Sigma\left(x_{r}-\bar{x}\right)^{2}+n(x-\bar{x})^{2} & \ldots \ldots .
\end{aligned}
$$

It follows that $\Sigma\left(x_{r}-x\right)^{2}$ is least when $x=\bar{x}$, or when $x$ coincides with the arithmetic mean of the observed values.

It will be convenient to use the abbreviation A.M. to denote the arithmetic mean.

The quantities obtained by subtracting the A.M. $\bar{x}$ from each of the observed values are called the residuals, and are usually denoted by $v_{1} v_{2}$, etc. We thus have a set of $n$ equations,

$$
\begin{aligned}
& x_{1}-\bar{x}=v_{1} \text {, } \\
& x_{2}-\bar{x}=v_{2} \text {, } \\
& x_{n}-\bar{x}=v_{n} . \\
& \bar{z}\left(x_{-}-x\right)^{2}=\vec{z} v_{-}^{2}
\end{aligned}
$$




$$
\text { Also } v_{1}+v_{2}+\ldots+v_{n}=\left(x_{1}+x_{2}+\ldots+x_{n}\right)-n \bar{x}=0
$$

The name of the method of "Least Squares" is due to the fact brought out by equation (2), that the most probable value of the unknown is that value for which the sum of the squares of the residuals is least.

In actual computation it is necessary to have numerical checks upon the calculated values of the A.M. and the sum of the squares of the residuals. If $\bar{x}$ be the A.M. and $x^{\prime}$ any other quantity, then

$$
\begin{aligned}
\bar{x} & =x^{\prime}+\frac{\sum\left(x_{r}-x^{\prime}\right)}{n}, \\
\sum v_{r}^{2} \quad \Sigma\left(x_{r}-\bar{x}\right)^{2} & =-n\left(\bar{x}-x^{\prime}\right)^{2}+\Sigma\left(x_{r}-x^{\prime}\right)^{2} .
\end{aligned}
$$

A calculation from some second base $x^{\prime}$ thus affords a useful check upon the value of the mean and upon the sum of the squares of the residuals. Also, in some cases, it may be more convenient to evaluate $\Sigma\left(x_{r}-x^{\prime}\right)^{2}$ than $\Sigma\left(x_{r}-\bar{x}\right)^{2}$.

The accuracy of the A.M. can also be checked by means of the sum of the residuals, since $\Sigma v=0$.

\section{Émuaturee \\ 14. Proof of the Normal Law from the Principle of the Arithmetic Mean.}

In $\S 13$ above we derived the principle of the arithmetic mean from the Normal Law. It is interesting to note that we can invert the process, and derive the Normal Law from the principle of the arithmetic mean.

Let the probability that the error of an observation should lie between $\Delta$ and $\Delta+d \Delta$ be represented by $\phi(\Delta) d \Delta$. Our problem is to find the form of the function $\phi(\Delta)$. As $d \Delta$ tends to zero $\phi(\Delta) d \Delta$ also tends to zero; or in other words, the probability of making an error of the exact value $\Delta$ is zero. When we speak of the "probability of an error $\Delta$," we shall interpret this expression as meaning the probability of an error between $\Delta-\alpha$ and $\Delta+\alpha$, where $\alpha$ is a small quantity which is just inappreciable in the observation in question. With this convention we may say that the probability of an error $\Delta$ is $C \phi(\Delta)$, where $C$ is a constant.

Let $x_{1}, x_{2}, \ldots, x_{n}$ be $n$ observed values of an unknown quantity $x$. If $x$ be the assumed value of the unknown, the errors of 
the separate observations will be $\Delta_{1}, \Delta_{2}, \ldots, \Delta_{n}$, defined by

$$
\Delta_{1}=x_{1}-x, \quad \Delta_{2}=x_{2}-x \text {, etc. }
$$

The probability of the occurrence of an error $\Delta_{r}$ is $C \phi\left(\Delta_{r}\right)$, and the probability of the occurrence of the system of errors $\Delta_{1}, \Delta_{2}, \ldots, \Delta_{n}$ is

$$
C^{n} \phi\left(\Delta_{1}\right) \phi\left(\Delta_{2}\right) \ldots \phi\left(\Delta_{n}\right)
$$

The assumed value of the unknown, $x$, must be the most probable value of the unknown; in other words, it will be such as to make the system of errors $\Delta_{1}, \Delta_{2}$, etc., the most probable. It follows that the probability

$$
C^{n} \phi\left(\Delta_{1}\right) \phi\left(\Delta_{2}\right) \ldots \phi\left(\Delta_{n}\right)
$$

must be a maximum for the assumed value of $x$. Differentiating with respect to $x$, we may write this condition in the form

$$
\begin{gathered}
\frac{\phi^{\prime}\left(\Delta_{1}\right)}{\phi\left(\Delta_{1}\right)} \frac{d \Delta_{1}}{d x}+\frac{\phi^{\prime}\left(\Delta_{2}\right)}{\phi\left(\Delta_{2}\right)} \frac{d \Delta_{2}}{d x}+\ldots+\frac{\phi^{\prime}\left(\Delta_{n}\right)}{\phi\left(\Delta_{n}\right)} \frac{d \Delta_{n}}{d x}=0, \\
\phi^{\prime}\left(\Delta_{r}\right)=\frac{d}{d r} \phi\left(\Delta_{r}\right) .
\end{gathered}
$$

where

But since

$$
\begin{gathered}
\Delta_{1}=x_{1}-x, \quad \Delta_{2}=x_{2}-x, \text { etc. } \\
\frac{d \Delta_{1}}{d x}=\frac{d \Delta_{2}}{d x}=\text { etc. }=-1,
\end{gathered}
$$

and the condition for a maximum may be written

or

$$
\begin{aligned}
\Sigma \frac{\phi^{\prime}\left(\Delta_{r}\right)}{\phi\left(\Delta_{r}\right)} & =0, \\
\Sigma \frac{\phi^{\prime}\left(\Delta_{r}\right)^{\prime}}{\Delta_{r} \phi\left(\Delta_{r}\right)} \Delta_{r} & =0 .
\end{aligned}
$$

Equation (1) must be satisfied for the most probable value of $x$.

We now assume that this value of $x$ is the A.M. With this assumption, we may write down the additional equation

$$
\Sigma \Delta_{r}=0
$$

which is simply equation (3) of $\S 13$.

Equations (1) and (2) must be simultaneously satisfied by the same value of the unknown, and so they must be identical.

$$
\begin{aligned}
\therefore \frac{\phi^{\prime}\left(\Delta_{1}\right)}{\Delta_{1} \phi\left(\Delta_{1}\right)} & =\frac{\phi^{\prime}\left(\Delta_{2}\right)}{\Delta_{2} \phi\left(\Delta_{2}\right)}=\ldots=\frac{\phi^{\prime}\left(\Delta_{n}\right)}{\Delta_{n} \phi\left(\Delta_{n}\right)} \\
& =\text { constant }=k \text { say } .
\end{aligned}
$$


Then

$$
\frac{\phi^{\prime}(\Delta)}{\Delta \phi(\Delta)}=k \quad \text { or } \frac{\phi^{\prime}(\Delta)}{\phi(\Delta)}=k \Delta
$$

yields a differential equation whose solution determines the form of $\phi(\Delta)$. Integrating the equation, we find

or

$$
\begin{aligned}
\log \phi(\Delta) & =\frac{1}{2} k \Delta^{2}+\text { const. } \\
\phi(\Delta) & =A e^{\frac{1}{2} k \Delta^{2}} .
\end{aligned}
$$

The product $\phi\left(\Delta_{1}\right) \phi\left(\Delta_{2}\right) \ldots \phi\left(\Delta_{n}\right)$ must be a maximum for the assumed value of $x$. Hence the series $\Sigma \log \phi\left(\Delta_{r}\right)$ must be a maximum. The condition to be satisfied is that

$$
\frac{d^{2}}{d x^{2}} \Sigma \log \phi(\Delta) \text { shall be negative, }
$$

or

$$
\Sigma \frac{d^{2}}{d x^{2}}\left(\frac{1}{2} k \Delta^{2}\right) \text { shall be negative. }
$$

Since

$$
\begin{gathered}
\Delta_{r}=x_{r}-x, \\
\frac{d^{2}}{d x^{2}} \Delta_{r}^{2}=2,
\end{gathered}
$$

and

$$
\Sigma \frac{d^{2}}{d x^{2}}\left(\frac{1}{2} k \Delta_{r}^{2}\right)=n \cdot k
$$

It follows that $k$ must be negative.

Putting $\frac{1}{2} k=-h^{2}$, we may write

$$
\phi(\Delta)=A e^{-h^{2} \Delta^{2}}
$$

The value of $A$ may be derived as in $\$ 8$, yielding

$$
\phi(\Delta)=\frac{h}{\sqrt{\pi}} e^{-h^{2} \Delta^{2}} .
$$

15. The Law of Error of a linear function of two independent quantities whose laws of error are known.

Let $m_{1}, m_{2}$ be two independent observed quantities, obeying the error laws

$$
\frac{h_{1}}{\sqrt{\pi}} e^{-h_{1}^{2} x^{2}} \text { and } \frac{h_{2}}{\sqrt{\pi}} e^{-h_{2}^{2} x^{2}} \text {, respectively. }
$$

The probability of the occurrence of an error between $x_{1}$ and $x_{1}+d x_{1}$ in $m_{1}$ is

$$
\frac{h_{1}}{\sqrt{\pi_{1}}} e^{-h_{1}{ }^{2} x_{1}{ }^{2}} d x_{1},
$$


and the probability of the occurrence of an error between $x_{2}$ and $x_{2}+d x_{2}$ in $m_{2}$ is

$$
\frac{h_{2}}{\sqrt{\pi}} e^{-h_{2}^{2} x_{2}^{2}} d x_{2}
$$

Since $m_{1}$ and $m_{2}$ are independent, the probability of the simultaneous occurrence of these errors in $m_{1}$ and $m_{2}$ is the product of the two separate probabilities. Calling this probability $p$ we have the equation

$$
p=\frac{h_{1} h_{2}}{\pi} e^{-h_{1}^{2} x_{1}^{2}-h_{2}^{2} x_{2}^{2}} d x_{1} d x_{2} .
$$

If the linear function in question be

$$
a_{1} m_{1}+a_{2} m_{2}=F,
$$

the corresponding error in $F$ will lie between

$$
a_{1} x_{1}+a_{2} x_{2}=x \text { (say) }
$$

and

$$
a_{1}\left(x_{1}+d x_{1}\right)+a_{2}\left(x_{2}+d x_{2}\right)=x+d x \text {. }
$$

An error $x$ in $F$ may be derived from any error $x_{1}$ in $m_{1}$, combined with the error $x_{2}$ in $m_{2}$; where $x_{2}$ is fixed by the relation

$$
a_{1} x_{1}+a_{2} x_{2}=x \text {. }
$$

Substituting for $x_{2}$, we find

$$
\begin{gathered}
p=\frac{h_{1} h_{2}}{\pi} e^{-h_{1}{ }^{2} x_{1}{ }^{2}-h_{2}{ }^{2}\left(\frac{x-a_{1} x_{1}}{a_{2}}\right)^{2}} d x_{1} d x_{2} \\
=\frac{h_{1} h_{2}}{\pi} e^{-\frac{h_{1}{ }^{2} h_{2}{ }^{2}}{a_{1}{ }^{2} h_{2}{ }^{2}+a_{2}{ }^{2} h_{1}{ }^{2}} x^{2}-\frac{a_{1}{ }^{2} h_{2}{ }^{2}+a_{2}{ }^{2} h_{1}{ }^{2}}{a_{2}{ }^{2}}\left(x_{1}-\frac{a_{1}{ }^{2} h_{2}{ }^{2}}{a_{1}{ }^{2} h_{2}{ }^{2}+a_{2}{ }^{2} h_{1}{ }^{2}} x\right)^{2}} d x_{1} d x_{2} .
\end{gathered}
$$

The probability of the occurrence of an error $x$ must take into account the fact that $x$ may be made up of any value of $x_{1}$ between $-\infty$ and $+\infty$, combined with the corresponding value of $x_{2}$ fixed by the equation

$$
x=a_{1} x_{1}+a_{2} x_{2} .
$$

This generality is obtained by integrating the function $p$ with respect to $x_{1}$, between the limits $\pm \infty$, afterwards taking into account the relation $d x=a_{2} d x_{2}$ which holds when $x_{1}$ has been eliminated. 
Let $\phi(x) d x$ be the probability of the occurrence of an error between $x$ and $x+d x$. Then, as above suggested,

$$
\begin{aligned}
& \phi(x) d x=\int_{-\infty}^{+\infty} p \\
= & \frac{h_{1} h_{2}}{\pi} d x_{2} e^{-\frac{h_{1}{ }^{2} h_{2}{ }^{2}}{a_{1} h_{2}{ }^{2}+a_{2}{ }^{2} h_{1}{ }^{2}} x^{2}} \int_{-\infty}^{+\infty} e^{-\frac{a_{1}{ }^{2} h_{2}{ }^{2}+a_{2}{ }^{2} h_{1}{ }^{2}}{a_{2}{ }^{2}}\left(x_{1}-\frac{a_{1}{ }^{2} h_{2}{ }^{2}}{a_{1}{ }^{2} h_{2}{ }^{2}+a_{2}{ }^{2} h_{1}{ }^{2}} x\right)^{2}} d x_{1} \\
= & \frac{h_{1} h_{2}}{\pi} d x_{2} e^{-\frac{h_{1}{ }^{2} h_{2}{ }^{2}}{a_{1}{ }^{2} h_{2}{ }^{2}+a_{2}{ }^{2} h_{1}{ }^{2}} x^{2}} \times \frac{\sqrt{\pi} a_{2}}{\sqrt{a_{1}{ }^{2} h_{2}{ }^{2}+a_{2}{ }^{2} h_{1}{ }^{2}}} .
\end{aligned}
$$

Taking into account the relation $d x=a_{2} d x_{2}$, we find

$$
\begin{aligned}
\phi(x) & =\frac{h_{1} h_{2}}{\sqrt{a_{1}^{2} h_{2}{ }^{2}+a_{2}{ }^{2} h_{1}^{2}}} \times \frac{1}{\sqrt{\pi}} e^{-\frac{h_{1}^{2} h_{2}{ }^{2}}{\sqrt{a_{1}^{2} h_{2}{ }^{2}+a_{2}{ }^{2} h_{1}^{2}}} x^{2}} \\
& =\frac{h}{\sqrt{\pi}} e^{-h^{2} x^{2}}
\end{aligned}
$$

where

$$
h=\frac{h_{1} h_{2}}{\sqrt{a_{1} h_{2}^{2}+a_{2}^{2} h_{1}^{2}}} .
$$

Thus the law of error of $F$ is of the same form as the laws of error of $m_{1}$ and $m_{2}$, and the parameter $h$ is fixed by the last equation. This equation may be written

$$
\frac{1}{h^{2}}=\frac{a_{1}^{2}}{h_{1}^{2}}+\frac{a_{2}^{2}}{h_{2}^{2}} .
$$

This formula is capable of generalisation for a linear function of any number of independent variables. Thus when

$$
F=a_{1} m_{1}+a_{2} m_{2}+a_{3} m_{3},
$$

if $h, h_{1}, h_{2}, h_{3}$ be the parameters of $F, m_{1}, m_{2}, m_{3}$, respectively, and $h_{2}{ }^{\prime}$ the parameter of the error law of the function $a_{2} m_{2}+a_{3} m_{3}$, we have the relations

and

$$
\begin{aligned}
\frac{1}{h_{2}{ }^{\prime 2}} & =\frac{a_{2}{ }^{2}}{h_{2}{ }^{2}}+\frac{a_{3}{ }^{2}}{h_{3}{ }^{2}}, \\
\frac{1}{h^{2}} & =\frac{a_{1}{ }^{2}}{h_{1}{ }^{2}}+\frac{1}{h_{2}{ }^{\prime 2}} .
\end{aligned}
$$

Hence we may write

$$
\frac{1}{h^{2}}=\frac{a_{1}^{2}}{h_{1}^{2}}+\frac{a_{2}^{2}}{h_{2}^{2}}+\frac{a_{3}^{2}}{h_{3}^{2}}
$$


In the same way, the result obtained above may be extended in succession to 4,5 , or any other number of independent variables. The final result may be stated thus:

If $F$ be a linear function of $n$ independent quantities, which have been determined by observation, the function $F$ follows an error law which is of the same form as the error laws of the independent unknowns. If the function is

$$
F=a_{1} m_{1}+a_{2} m_{2}+\ldots+a_{n} m_{n},
$$

the parameter $h$ of the function $F$ is given by

$$
\frac{1}{h^{2}}=\frac{a_{1}^{2}}{h_{1}^{2}}+\frac{a_{2}^{2}}{h_{2}^{2}}+\ldots+\frac{a_{n}^{2}}{h_{n}^{2}}=\left[\frac{a^{2}}{h^{2}}\right] .
$$

\section{The Median.}

The median, which is the value of the unknown which has as many observed values on one side of it as on the other, is the natural competitor of the arithmetic mean, and it is interesting to consider the law of error which follows from the assumption that the median is the most probable value of the unknown.

Let $x^{\prime}$ be the median, and let $f\left(x-x^{\prime}\right) d x$ be the probability of making an observation between $x$ and $x+d x$. If $x_{1}, x_{2}, \ldots, x_{n}$ be the observed values, we have to make the product

$$
f\left(x_{1}-x^{\prime}\right) f\left(x_{2}-x^{\prime}\right) \ldots f\left(x_{n}-x^{\prime}\right) \text { a maximum, }
$$

or the sum

$$
\Sigma \log f\left(x_{r}-x^{\prime}\right) \text { a maximum. }
$$

Differentiating with respect to $x^{\prime}$, we obtain the condition for a maximum in the form

$$
\Sigma \frac{f^{\prime}}{f}=0
$$

Let

$$
F(u)=\frac{f^{\prime}}{f},
$$

where $u$ is the error $x_{r}-x^{\prime}$.

Then $\Sigma F(u)=0$ whenever the numbers of positive and negative errors are equal. This is satisfied by making

$$
F(u)= \pm k \text {, }
$$

where the upper or lower sign is to be taken according as $u$ is positive or negative. 
Writing

and integrating, we find

$$
\frac{f^{\prime}}{f}= \pm k
$$

$$
\log f(u)= \pm k u+\text { constant }=k|u|+\text { constant }
$$

and

$$
f(u)=C e^{k|u|} \text {. }
$$

Since the probability must decrease as $u$ increases it follows that $k$ is negative, and the form of the error law is

$$
f(u)=C e^{-l^{2}|u|} \text {. }
$$

Since any error must lie between $-\infty$ and $+\infty$, we have, as in the case of the Normal Law,

$$
1=\int_{-\infty}^{+\infty} f(u) d u=2 C \int_{0}^{\infty} e^{-l^{2} u} d u=\frac{2 C}{l^{2}},
$$

and finally we may write

$$
f(u)=\frac{l^{2}}{2} e^{-l^{2}|u|}
$$

\section{EXAMPLES.}

1. Show that the average value of the error $(\eta$ of page 30$)$ is $\frac{1}{l^{2}}$ when the above law holds.

2. Show that the above law yields as the most probable value of the unknown that which makes the arithmetic sum of the errors a minimum.

3. Given that the arithmetic sum of the errors is to be a minimum, show that the median is the most probable value of the unknown.

4. Find the P.E. $s$, a single observation.

$$
\left[e^{-l^{2} 8}=\frac{1}{2} .\right]
$$

\section{REFERENCES.}

For a full discussion of a number of attempts at proving the Normal Error Law the reader is referred to Glaisher, Memoirs of the Royal Astronomical Society, 1872.

The earlier work on the subject will be found in Todhunter, History of Probability.

Gauss's contributions to the subject will be found in: Theoria Motus Corporum Coelestium, Lib. II, Sect. III ; Theoria Combinationis Observationum Erroribus Minimis Obnoxiae, Comment. Soc. Göttingen, Vol. v, 1819-1822; Supplementum Theoriae Combinationis Observationum, ibid., Vol. vI, 18231827. 


\section{CHAPTER III}

\section{THE CASE OF ONE UNKNOWN}

\section{Measures of Precision.}

The area bounded by the axis of $x$, and the curve

$$
y=\frac{h}{\sqrt{\pi}} e^{-h^{2} x^{2}},
$$

is unity for all values of $h$. The greater the value of $h$, the greater is the central ordinate, and the steeper the curve; i.e. the greater the value of $h$, the more closely will the observations be clustered about the mean value. If two sets of observations of the same quantity be made, the series for which $h$ is greater will be more closely clustered about the mean value, and may therefore be regarded as a better set of observations than the set for which $h$ is less. Hence $/ h$ may be regarded as a measure of the precision of the observations, and may be used as a criterion for judging the accuracy with which the observations have been carried out.

In practice it is found more convenient to use certain funetions of $h$, rather than $1 / \hbar$ itself, as measures of precision. Most continental writers use a function which is termed the mean square error or M.S.E., a term which is loosely used to denote the square root of the average value of the squares of the errors of observation. It is usually denoted by the Greek letter $\mu$. "Usuaily calle) "rost mem Se pare.

Of a set of $n$ observations, the number whose errors will lie between $\Delta$ and $\Delta+d \Delta$ will be

$$
\frac{n h}{\sqrt{\pi}} e^{-h^{2} \Delta^{2}} d \Delta
$$

The sum of the squares of these errors will be

$$
\frac{n h}{\sqrt{\pi}} \Delta^{2} e^{-h^{2} \Delta^{2}} d \Delta \text {. }
$$


If $\mu$ be the M.S.E., it follows that the sum of the squares of all the errors from $-\infty$ to $+\infty$

$$
\begin{aligned}
& =n \mu^{2}=\frac{n h}{\sqrt{\pi}} \int_{-\infty}^{+\infty} e^{-h^{2} \Delta^{2} \Delta^{2} d \Delta} \\
& =\frac{2 n h}{\sqrt{\pi}} \int_{0}^{\infty} e^{-h^{2} \Delta^{2} \Delta^{2} d \Delta} \\
& =\frac{n}{2 h^{2}} . \\
& \therefore \mu^{2}=\frac{1}{2 h^{2}} \text { and } \mu=\frac{1}{\sqrt{2} h} .
\end{aligned}
$$

English and American writers generally use as a measure of precision a quantity $r$, unhappily named the probable error or P.E.* The P.E., $r$, is of such a magnitude that the error of a single observation is as likely to be within as without the limits $\pm r$. Or, to express it in another way, the odds are even that the error of a single observation shall not be greater in magnitude than $r$. This condition is expressed mathematically by the equation

$$
\frac{1}{2}=\frac{h}{\sqrt{ } \pi} \int_{-r}^{+r} e^{-h^{2} x^{2}} d x=\frac{2}{\sqrt{\pi}} \int_{0}^{h r} e^{-x^{2}} d x .
$$

This equation can be solved by the use of tables of the integral $\int_{0}^{x} e^{-x^{2}} d x$, yielding the result

$$
h r=0.47696=\rho .
$$

The quantity $\rho$ is the one mentioned on page 19 .

It follows that

$$
r=\frac{0.47696}{h}=0.6745 \mu=\frac{2}{3} \mu \text { (approximately). }
$$

The P.E. has a very simple meaning, in that one-half of the observations in a series should have errors greater than $r$, and the other half should have errors less than $r$.

Another measure of precision which is sometimes used is the average error $\eta$, whose value is the average of the errors of all the

\footnotetext{
* The most probable error is zero, corresponding to the highest point of the error curve.
} 
observations, considered without regard to sign. Its relation to $h$ can be easily derived.

$$
\begin{aligned}
n \eta & =\frac{n h}{\sqrt{\pi}} \int_{0}^{\infty} x e^{-h^{2} x^{2}} d x+\frac{h}{\sqrt{ } \pi} \int_{0}^{-\infty}(-x) e^{-h^{2} x^{2}} d x . \\
\therefore \eta & =\frac{2 h}{\sqrt{\pi}} \int_{0}^{\infty} x e^{-h^{2} x^{2}} d x \\
& =\frac{1}{h \sqrt{ } \pi} .
\end{aligned}
$$

It follows that

$$
\mu=\eta \times \sqrt{\frac{\pi}{2}},
$$

and

\begin{tabular}{|c|c|c|c|c|}
\hline . & $\frac{1}{h}$ & $=\begin{array}{c}\mu \\
=-\sqrt{3} 2 \\
\end{array}$ & $\begin{aligned} & r \\
= & p / h\end{aligned}$ & $\begin{array}{c}\eta \\
4\end{array}$ \\
\hline$\frac{1}{h}$ & $1 \cdot 0000$ & $1 \cdot 4142$ & $2 \cdot 0966$ & $1 \cdot 7726$ \\
\hline$\mu=\frac{1}{1 L h}$ & $\cdot 7071$ & $1 \cdot 0000$ & $1 \cdot 4826$ & $1 \cdot 2533$ \\
\hline$r=\frac{P}{L}$ & $\cdot 4769$ & $\cdot 6745$ & $1 \cdot 0000$ & $\cdot 8453$ \\
\hline$\eta \frac{1}{4 \pi}$ & $\cdot 5642$ & $\cdot 7979$ & $1 \cdot 829$ & $1 \cdot 0000$ \\
\hline
\end{tabular}

$$
r=0.6745 \mu=0.8453 \eta \text {. }
$$

The relations of $\frac{1}{h}, r, \mu$, and $\eta$ are here collected in tabular form for convenience of reference.

The following approximate values of some of the above coefficients are occasionally of use :

$$
\begin{aligned}
& \cdot 6745=\frac{2}{3} . \\
& \cdot 8453=\frac{14}{13} . \\
& \cdot 47696=\frac{10}{21} .
\end{aligned}
$$

\section{Evaluation of $h$ and $r$.}

The relation between $h$ and the residuals can be obtained as follows. Let $n$ similarly observed values of a quantity be $x_{1}, x_{2}, x_{3}, \ldots, x_{n}$, and let $X$ be the true value of the quantity. 
Then the errors of the separate observations are $x_{1}-X, x_{2}-X$, $\ldots, x_{n}-X$. The probability of an error lying between $x$ and $x+d x$ is

$$
\frac{h}{\sqrt{\pi}} e^{-h^{2} x^{2}} d x
$$

As on page 22 this may be expressed by saying that the probability of an error $x$ is $C h e^{-h^{2} x^{2}}$, where $C$ is a constant. Then the a priori probability of the occurrence of the system of errors $x_{1}-X, x_{2}-X$, etc. is

or

$$
\begin{gathered}
C^{n} h^{n} e^{-h^{2} \Sigma\left(x_{r}-X\right)^{2},} \\
C^{n} h^{n} e^{-h^{2} \Sigma v_{r}^{2}} e^{-h^{2} n(M-X)^{2}},
\end{gathered}
$$

where

$$
M=\frac{\Sigma x}{n} \text { and } v_{r}=x_{r}-M .
$$

The true value of $X$ is unknown. All that can definitely be said is that it lies between $-\infty$ and $+\infty$. Thus the probability of the given system of measurements $x_{1}, x_{2}, \ldots, x_{n}$ must be written as the integral

$$
C^{n} h^{n} e^{-h^{2} \Sigma v^{2}} \int_{-\infty}^{+\infty} e^{-h^{2} n(M-X)^{2}} d X, 7=\frac{1}{2} \sqrt{\frac{\pi}{m}}
$$

and this can immediately be reduced to

$$
C^{n} h^{n-1} \sqrt{\frac{\pi}{n}} e^{-h^{2} \Sigma v^{2}}
$$

The value of $h$ must be such as to make this probability a maximum. Taking logarithms and differentiating with respect to $h$, we find

or

$$
\begin{aligned}
& \frac{n-1}{h}-2 h \Sigma v^{2}=0, \\
& h^{2}=\frac{n-1}{2 \Sigma v^{2}}=\frac{n-1}{2[v v]} .
\end{aligned}
$$

In writings on the present subject it is customary to denote summation, not by the letter $\Sigma$, but by square brackets. The last equation reduces to

It immediately follows that

$$
h=\sqrt{\frac{n-1}{2[v v]}} .
$$

$$
r=0.47696 \frac{1}{h}=0.6745 \sqrt{\frac{[v v]}{n-1}} .
$$


19. Comparison of a set of observations with the preceding theory.

Gauss (Werke, Iv. p. 116) took Bessel's reduction of 470 observations of the right ascensions of Procyon and Altair made by Bradley, and compared the distribution of errors with the theoretical curve obtained by evaluating $h$ by the above formula. He calculated the numbers of observations whose errors should be numerically between $0^{\prime \prime} \cdot 0$ and $0^{\prime \prime} \cdot 1$, between $0^{\prime \prime} \cdot 1$ and $0^{\prime \prime} \cdot 2$, etc., and compared them with the actual numbers obtained from Bradley's observations. The results are given in the following table.

\begin{tabular}{|c|c|c|}
\hline Errors & $\begin{array}{c}\text { Theoretical } \\
\text { number }\end{array}$ & $\begin{array}{c}\text { Actual } \\
\text { number }\end{array}$ \\
\hline $0^{\prime \prime} \cdot 0-0^{\prime \prime} \cdot 1$ & $94 \cdot 8$ & 94 \\
$0^{\prime \prime} \cdot 1-0^{\prime \prime} \cdot 2$ & $88 \cdot 8$ & 88 \\
$0^{\prime \prime} \cdot 2-0^{\prime \prime} \cdot 3$ & $78 \cdot 3$ & 78 \\
$0^{\prime \prime} \cdot 3-0^{\prime \prime} \cdot 4$ & $64 \cdot 1$ & 58 \\
$0^{\prime \prime} \cdot 4-0^{\prime \prime} \cdot 5$ & $49 \cdot 5$ & 51 \\
$0^{\prime \prime} \cdot 5-0^{\prime \prime} \cdot 6$ & $35 \cdot 8$ & 36 \\
$0^{\prime \prime} \cdot 6-0^{\prime \prime} \cdot 7$ & $24 \cdot 2$ & 26 \\
$0^{\prime \prime} \cdot 7-0^{\prime \prime} \cdot 8$ & $15 \cdot 4$ & 14 \\
$0^{\prime \prime} \cdot 8-0^{\prime \prime} \cdot 9$ & $9 \cdot 1$ & 10 \\
$0^{\prime \prime} \cdot 9-1 " \cdot 0$ & $5 \cdot 0$ & 7 \\
above $1^{\prime \prime} \cdot 0$ & $5 \cdot 0$ & 8 \\
\hline
\end{tabular}

The table shows a remarkable correspondence between the theory and the observational data. There is, however, a slight discrepancy in the number of large errors, the number occurring in practice exceeding the theoretical number. This discrepancy occurs in other series of observations, and some attempt to deal with it will be made in a later chapter.

20. Evaluation of $\mu$.

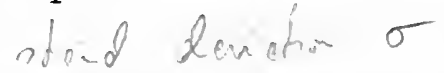

The quantity $\mu$, the M.S. E. of a system of errors, is connected with $h$ by the relation

$$
\boldsymbol{\mu}=\frac{1}{h \sqrt{2}} \text {. sterbad devistis } \sigma
$$

It has already been shown that $h=\sqrt{\frac{n-1}{2[v v]}}$, and so $\mu$ should be given by

$$
\mu=\sqrt{\frac{[v v]}{n-1}} \text {. }
$$

B. 0 . 
This relation is of such importance that it is necessary to consider it in some detail. The residuals $v_{1}, v_{2}$, etc. are the deviations of the observed values from the A.M., and if the A.M. could be definitely regarded as the true value of the unknown, the (M.S.E.) $)^{2}$ ought to be equal to $\frac{[v v]}{n}$. The use of the denominator $n-1$ instead of $n$ must be regarded as due to the uncertainty as to the true value of the unknown. The derivation of the formula for $\mu$ from a purely algebraic standpoint may help to elucidate the question.

Let $x_{1}, x_{2}, \ldots, x_{n}$ be the $n$ observed values. Now suppose these to be the first $n$ observations in a series of $N$ observations. Let $V_{1}, V_{2}, \ldots, V_{N}$ be the residuals calculated from the mean of the $N$ observations.

Let

$$
\mu^{2}=\frac{[V V]}{N}, \quad \mu^{\prime 2}=\frac{[v v]}{n} .
$$

If $v_{s}$ be the residual of the $s$ th observation within the group of $n$ observations, then

$$
v_{s}=d+V_{s}
$$

where $d$ is difference between the mean of the $N$ observations and the mean of the group of $n$. The value of $d$ is given by

$$
d=\frac{V_{1}+V_{2}+\ldots+V_{n}}{n}
$$

Squaring equation (2) we find

$$
d^{2}=\frac{1}{n^{2}} \sum_{1}^{n} V_{s}^{2}+\frac{2}{n^{2}} \Sigma V_{8} V_{t} \text {. }
$$

There will be $\frac{1}{2} n(n-1)$ terms of the form $V_{s} V_{t}$, and the equation may be written

$$
d^{2}=\frac{1}{n^{2}} \sum_{1}^{n} V_{s}^{2}+\frac{n-1}{n} \times \text { mean value of } V_{\delta} V_{t}
$$

If a large number of different groups of $n$ be selected from among the total of $N$ observations, and equation (3) be formed for each group, we can write down the mean value of each side of equation (3) for all these groups.

The resulting equation is

$$
\bar{d}^{2}=\frac{\mu^{2}}{n}+\frac{n-1}{n} \times \text { inean value of } V_{s} V_{t}
$$

where $\bar{d}^{2}$ is the mean value of $d^{2}$. 
But $0=\left(V_{1}+V_{2}+\ldots+V_{N}\right)^{2}$

$$
=[V V]+N(N-1) \times \text { mean value of } V_{s} V_{t} \text {. }
$$

$\therefore$ mean value of $V_{s} V_{t}=-\frac{[V V]}{N(N-1)}=-\frac{\mu^{2}}{N-1} \ldots\left(4^{\prime}\right)$.

Substituting this result in equation (4) we find

$$
\bar{d}^{2}=\frac{\mu^{2}}{n}-\frac{n-1}{n} \frac{\mu^{2}}{N-1}=\frac{\mu^{2}(N-n)}{n(N-1)}
$$

Now

$$
V_{8}^{2}=\left(d+v_{8}\right)^{2}=d^{2}+2 d v_{8}+v_{8}^{2} .
$$

There will be $n$ such equations, and if we write these $n$ equations down and take the mean value of both sides, we find

$$
\text { mean value of } V_{8}^{2}=d^{2}+\mu^{\prime 2}
$$

If we form equation (6) for.all the possible sets of $n$ observations, and then take the mean of all these equations, we find

$$
\mu^{2}=\bar{d}^{2}+\mu^{\prime 2},
$$

or, substituting for $\bar{d}^{2}$ from equation (5),

$$
\begin{aligned}
& \mu^{2}=\frac{\mu^{2}}{n} \frac{N-n}{N-1}+\mu^{\prime 2} \\
& \mu^{\prime 2}=\mu^{2} \frac{n-1}{n} \frac{N}{N-1}
\end{aligned}
$$

If we have a very large number $N$ of observations, and we take samples of $n$ at a time, from among the number $N$, the average value of the (mean-square-residual) ${ }^{2}$ for all the possible samples is connected with the mean square residual of all the $N$ observations $(\mu)$, by equation ( 7$)$.

If $N$ be regarded as becoming an indefinitely large number, the mean of the $N$ observed values may be taken as the true value of the unknown, and $\mu$ is then the M.S.E. of a single observation. Equation (7) then yields

$$
\begin{aligned}
\mu^{2}=\frac{n}{n-1} \mu^{\prime 2} & =\frac{n}{n-1} \frac{[v v]}{n}=\frac{[v v]}{n-1} . \\
\mu & =\sqrt{\frac{[v v]}{n-1}} .
\end{aligned}
$$

Whence

The formula $(n-1) \mu^{2}=[v v]$ may be interpreted as follows. If we take an infinite series of observations of any quantity, and select a large number of samples of $n$ observations, the sum of 
the squares of the $n$ residuals calculated from the mean of the $n$ observations will have the mean value $(n-1) \mu^{2}$. The factor $n-1$ is due to the fact that the mean value of the observations in any one sample is liable to differ from the true value of the unknown.

21. Probable error and mean square error of the arithmetic mean.

Returning to equation (5) above, $\bar{d}$ is the M. S. E. of the mean of $n$ observations. If we take $N$ to be an infinitely large number, $\mu$ becomes the u.S. E. of a single observation.) Equation (5) may then be written

$$
\bar{d}^{2}=\frac{\mu^{2}}{n} \text { or } \bar{d}=\frac{\mu}{\sqrt{n}}
$$

$\mathrm{Or}$

$$
\text { M. S. E. of A. M. }=\frac{\text { M.S.E. of a single observation }}{\sqrt{n}} \text {. }
$$

Since the relation $r=0.6745 \mu$ is always true, we obtain immediately the result

$$
\begin{aligned}
\text { P.E. of A. M. } & =\frac{\text { P. E. of a single observation }}{\sqrt{n}}=\frac{r}{\sqrt{n}} \\
& =0.6745 \sqrt{\frac{[v v]}{n(n-1)}} .
\end{aligned}
$$

22. Probable error of a linear function of a number of independent quantities whose probable errors are known*.

In the first case we shall consider a linear function of two independent quantities $m_{1}, m_{2}$, whose mean square errors are $\mu_{1}, \mu_{2}$. Let the linear function be

$$
F=a_{1} m_{1}+a_{2} m_{2}
$$

where $a_{1}$ and $a_{2}$ are constants.

If an error $x$ be made in determining $m_{1}$, and an error $y$ in determining $m_{2}$, the corresponding error $d F$ in $F$ is given by

$$
d F=a_{1} x+a_{2} y \text {. }
$$

Squaring this equation, we obtain

$$
d F^{2}=a_{1}^{2} x^{2}+a_{2}^{2} y^{2}+2 a_{1} a_{2} x y .
$$

* This proof assumes that the error law of a linear function of a number of independent quantities is of the same form as the error law of each of these quantities. Vide $\S 15$ for proof of this assumption. 
This equation holds for all values of $x$ and $y$; and therefore the mean value of the L.H.S. is equal to the mean value of the R.H.S. But if $\mu, \mu_{1}, \mu_{2}$ be the M. S. E.'s of $F, m_{1}$, and $m_{2}$, respectively, the equation leads to

$$
\mu^{2}=a_{1}^{2} \mu_{1}^{2}+a_{2}^{2} \mu_{2}^{2}+2 a_{1} a_{2} \times \text { mean value of } x y .
$$

Since $m_{1}$ and $m_{2}$ are independent, the errors $x$ and $y$ are also independent. With a given value of $x$, positive and negative values of $y$ are equally likely to be associated; and so the mean value of the product $x y$ is zero.

$$
\therefore \mu^{2}=a_{1}^{2} \mu_{1}^{2}+a_{2}^{2} \mu_{2}^{2} \text {. }
$$

This result may be extended to apply to any number of variables, the proof being the same as that given above for two variables. In general, if

$$
F=a_{1} m_{1}+a_{2} m_{2}+\ldots+a_{n} m_{n},
$$

where $a_{1}, a_{2}, \ldots, a_{n}$ are constant, then

$$
\mu^{2}=a_{1}^{2} \mu_{1}^{2}+a_{2}^{2} \mu_{2}^{2}+\ldots+a_{n}^{2} \mu_{n}^{2},
$$

or, if $r, r_{1}, r_{2}, \ldots, r_{n}$ be the P.E.'s of $F, m_{1}, m_{2}, \ldots, m_{n}$,

$$
r^{2}=a_{1}^{2} r_{1}^{2}+a_{2}^{2} r_{2}^{2}+\ldots+a_{n}^{2} r_{n}^{2} .
$$

\section{Probable error of the Arithmetic Mean.}

The P.E. of the A.M. can be immediately derived from the above. For if $x_{1}, x_{2}, \ldots, x_{n}$ be $n$ independently observed values, each with P.E. $r$, and if $r^{\prime}$ be the P.E. of the A.M. $\bar{x}$, then

$$
\bar{x}=\frac{1}{n}\left(x_{1}+x_{2}+\ldots+x_{n}\right)
$$

and

$$
r^{\prime 2}=\frac{1}{n^{2}}\left(r^{2}+r^{2}+\text { etc. to } n \text { terms }\right)=\frac{r^{2}}{n} \text {. }
$$

The P. E. of the A.M. is therefore $\frac{r}{\sqrt{n}}$.

If $\mu$ be the M.S.E. of a single observation, the M.S.E. of the A. M. is $\frac{\mu}{\sqrt{n}}$.

Exercise. Derive the above results from the formulae given at the end of $\S 15$. 


\section{Peters' Formula for $\mathbf{r}$.}

Let $x_{1}, x_{2}, \ldots, x_{n}$ be $n$ observed values of a quantity whose true value is $x$. Then the true errors $\epsilon_{1}, \epsilon_{2}, \ldots, \epsilon_{n}$ are given by the equations

$$
x_{1}=x-\epsilon_{1}, \quad x_{2}=x-\epsilon_{2}, \text { etc. }
$$

The A. M. of the observed values is

or

$$
\begin{gathered}
\frac{1}{n}\left(x_{1}+x_{2}+\ldots+x_{n}\right), \\
x-\frac{1}{n}\left(\epsilon_{1}+\epsilon_{2}+\ldots+\epsilon_{n}\right) .
\end{gathered}
$$

If $v_{1}, v_{2}$, etc. be the deviations of $x_{1}, x_{2}$, etc. from the mean,

$$
\begin{aligned}
& v_{1}=\frac{1}{n}\left\{(n-1) \epsilon_{1}-\epsilon_{2}-\epsilon_{3}-\ldots-\epsilon_{n}\right\}, \\
& v_{2}=\frac{1}{n}\left\{-\epsilon_{1}+(n-1) \epsilon_{2}-\epsilon_{3}-\ldots-\epsilon_{n}\right\}, \text { etc. }
\end{aligned}
$$

All the $n$ observations are supposed to be liable to the same errors, or are supposed to follow the same error law. And since the residuals $v_{1}, v_{2}$, etc. are linear functions of $\epsilon_{1}, \epsilon_{2}$, etc., it follows from $\S 15$ that the residuals are subject to a similar error law, the parameter $h$ being the same for all the residuals, since the sum of the squares of the coefficients on the R.H.S. is the same for all $v$ 's.

Let the P. E. of each $\epsilon$ be $r$, and let the P. E. of any residual $v$ be $r^{\prime}$. Then it follows from $\S 22$ that

$$
r^{\prime 2}=\frac{r^{2}}{n^{2}}\left\{(n-1)^{2}+(n-1)\right\}=r^{2} \frac{n-1}{n}
$$

Hence the true P.E. $r$ of the observations is $r^{\prime} \sqrt{\frac{n}{n-1}}$, where $r^{\prime}$ is the P.E. derived from the residuals.

If $v$ ] be the sum of the residuals, taken without regard to sign, then

$$
\begin{aligned}
& \quad r^{\prime}=0.8453 \frac{v]}{n} . \\
& \therefore \quad r=0.8453 \frac{v]}{\sqrt{n(n-1)}}
\end{aligned}
$$

Equation (2) is known as Peters' formula for the probable error. 
It should be noted that equation (1) above leads also to the ordinary formula for the P. E. derived from the squares of the residuals. The $\mathbf{P}$. E. of a residual

$$
\begin{gathered}
=r^{\prime}=0.6745 \sqrt{\frac{[v v]}{n}} \\
\therefore r=r^{\prime} \sqrt{\frac{n}{n-1}}=0.6745 \sqrt{\frac{[v v]}{n-1}}
\end{gathered}
$$

We thus have two equations for the evaluation of the P.E. of a single observation from the residuals.

\section{Some Examples of the Adjustment of Observa- tions of one unknown.}

Example 1. The heat of evaporation of water*.

Each of the 20 values given in the first eolumn of the table below is an independent determination of the heat of evaporation of water. It is required to find the adjusted value, and its P.E. The A.Mr. of all the observations is adopted as the most probable value. The residuals obtained by subtracting the A.s. from each determination are written in the second column, the positive and negative residuals being separated into sub-columns. The $\frac{1}{4}$ squares

\begin{tabular}{|c|c|c|}
\hline Observed value & Residual & 1 square of residual \\
\hline $\begin{array}{r}542 \cdot 98 \\
1 \cdot 23 \\
0 \cdot 64 \\
2 \cdot 03 \\
2 \cdot 32 \\
1 \cdot 48 \\
2 \cdot 37 \\
2 \cdot 15 \\
1 \cdot 36 \\
1 \cdot 34 \\
2 \cdot 91 \\
2 \cdot 68 \\
3 \cdot 08 \\
2 \cdot 12 \\
1 \cdot 82 \\
0 \cdot 96 \\
1 \cdot 66 \\
1 \cdot 73 \\
1 \cdot 79 \\
1 \cdot 83\end{array}$ & $\begin{array}{cc}+ & - \\
1 \cdot 056 & \cdot \\
& \cdot 694 \\
\cdot 106 & 1 \cdot 284 \\
\cdot 396 & \\
& \cdot 444 \\
\cdot 446 & \\
\cdot 226 & \\
& \cdot 564 \\
& \cdot 584 \\
\cdot 986 & \\
\cdot 756 & \\
1 \cdot 156 & \\
\cdot 196 & \\
& \cdot 104 \\
& \cdot 964 \\
& \cdot 264 \\
\cdot & \cdot 194 \\
& \cdot 134 \\
& \cdot 094\end{array}$ & $\begin{array}{l}\cdot 2788 \\
\cdot 1204 \\
\cdot 4122 \\
\cdot 0028 \\
\cdot 0392 \\
\cdot 0493 \\
\cdot 0497 \\
\cdot 0128 \\
\cdot 0795 \\
\cdot 0853 \\
\cdot 2430 \\
\cdot 1429 \\
\cdot 3341 \\
\cdot 0096 \\
\cdot 0027 \\
\cdot 2323 \\
\cdot 0174 \\
\cdot 0094 \\
\cdot 0045 \\
\cdot 0022\end{array}$ \\
\hline Mean $541 \cdot 924$ & $+5 \cdot 324 \quad-5 \cdot 324$ & $2 \cdot 1281$ \\
\hline
\end{tabular}
of the residuals are written in the last eolurnn.

* A. W. Smith, Plyysical Review, September, 1911. 
The correspondence of the sums of positive and negative residuals affords a check on the value of the $\mathrm{A}$. $\mathrm{s}$.

$$
[v v]=2 \cdot 1281 \times 4=8 \cdot 5124 .
$$

Using the formulae of pages 32 and 36 , we find

$$
\begin{gathered}
\text { P.E. of a single observation }=\cdot 6745 \sqrt{\frac{8 \cdot 5124}{19}}=\cdot 451, \\
\text { P.E. of A. M. }=\frac{\cdot 451}{\sqrt{20}}=\cdot 101 .
\end{gathered}
$$

Using Peters' formula we find

$$
\begin{gathered}
\text { P. E. of a single observation }=\cdot 8453 \times \frac{10 \cdot 648}{\sqrt{20 \times 19}}=\cdot 462, \\
\text { P.E. of A. M. }=\frac{\cdot 462}{\sqrt{20}}=\cdot 103 .
\end{gathered}
$$

The two formulae for P.E. yield almost identical results. The final value of the unknown, together with its P.E., can be represented as

\section{$541 \cdot 924 \pm \cdot 102$.}

The residuals of column 2 are shown in figure 4 . The whole range of variation is divided up into intervals of 4 , the middle interval extending

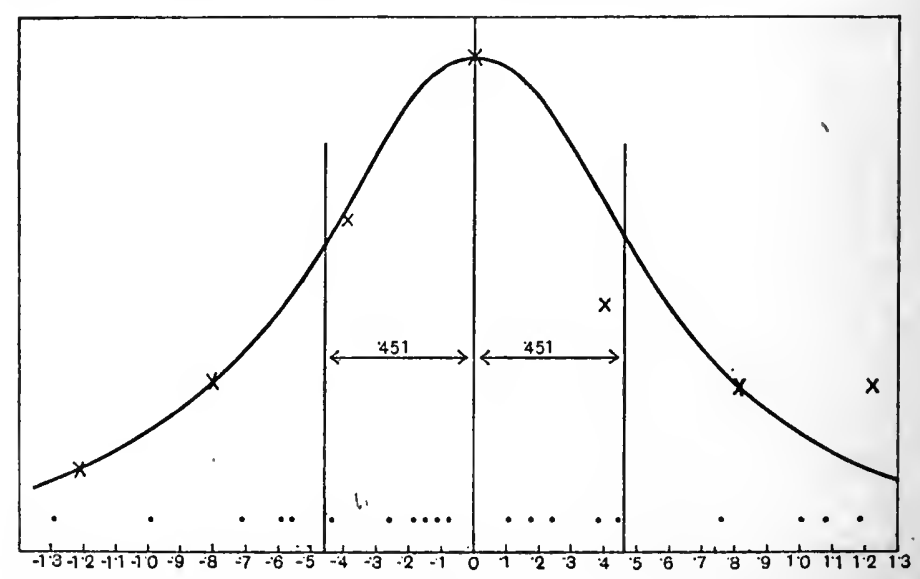

Fig. 4.

from -2 to $+\cdot 2$. In the diagram a dot is put in to represent, as accurately as possible, the value of each residual. An ordinate is erected at the middle of each interval, of a length proportional to the number of observations falling within that interval. The top of each ordinate is represented by $a \times$. A smooth symnetrical curve is drawn to fit the tops as closely as possible. Although two of the points representing positive residuals are at some distance from the curve, it is seen that the general form of the curve is in fair agreement with the Gauss error curve shown in figure 3. The discrepancy is possibly due to the smallness of the number of determinations used in the reduction. 
In the diagram two ordinates are erected at distances $\pm \cdot 451$ from the origin. According to the least square theory, 10 observed values should lie between these ordinates, and 10 outside these limits. It is seen that 11 observed values lie within the given linits, and 9 without; a result in sufficiently good agreement with the theory, in view of the relative smallness of the number of determinations.

\section{Example 2. The atomic weight of bromine*.}

The ratio of the weights of Bromine and Hydrogen which combine to form Hydrobromic acid was determined experimentally. The results of 10 independent determinations are given in the first column of the table below.

\begin{tabular}{|c|c|c|c|}
\hline Observed value & \multicolumn{2}{|c|}{ Residual } & $\frac{1}{4}$ square of residual \\
\hline $\begin{array}{r}79 \cdot 2863 \\
\cdot 3055 \\
\cdot 3064 \\
\cdot 3197 \\
\cdot 3114 \\
\cdot 3150 \\
\cdot 3063 \\
\cdot 3141 \\
\cdot 2915 \\
\cdot 3108\end{array}$ & $\begin{array}{c}+ \\
\\
.0130 \\
.0047 \\
.0083 \\
.0074 \\
.0041\end{array}$ & $\begin{array}{c}- \\
.0204 \\
.0012 \\
.0003\end{array}$ & $\begin{array}{r}.00010404 \\
36 \\
2 \\
4225 \\
552 \\
1722 \\
4 \\
1369 \\
5776 \\
420\end{array}$ \\
\hline Mean $79 \cdot 3067$ & $\cdot 0375$ & 0375 & .00024510 \\
\hline
\end{tabular}

$$
[v v]=\cdot 0009804, \quad v]=\cdot 075,
$$

P. E. of a single determination $=\sqrt{\frac{0009804}{9}} \times 6745=\cdot 0070$,

$$
\text { P. E. of the A.M. }=\frac{\cdot 0070}{\sqrt{10}}=\cdot 0022 \text {, }
$$

P.E. of A.M. from Peters' formula $=\frac{1}{\sqrt{10}} \times \cdot 8453 \times \frac{\cdot 075}{\sqrt{90}}=\cdot 0021$.

The adjusted value of the ratio is thus $79 \cdot 3067 \pm 002$.

The atomic weight of Hydrogen $=1.00779$.

Therefore the atomic weight of Bromine

$$
=1 \cdot 00779(79 \cdot 3067 \pm \cdot 002)=79 \cdot 924 \pm \cdot 002 .
$$

* Weber, Bulletin of Bureau of Standards, Vol. Ix, p. 131. 


\section{Example 3. The percentage of dry matter in mangel roots*.}

The percentage of dry matter was estimated for each of 160 roots of a strain of Golden Globe mangel. The results varied between 10.7\% and 19.7\%, and the A.M. of all the results gave $14.5 \%$. It was necessary to consider whether it was justifiable to take the A.M. of such widely differing results, and if so, what was its precision. In order to find the answer to this question we must first consider what are the different causes which tend to produce differences in content of dry matter in individual roots; and whether these causes satisfy the assumptions made on page 5 as to the nature of accidental errors.

All the roots taken were of the same strain, grown side by side, and sampled and analysed in the same manner. Thus the possibilities of variation in ,individual roots were reduced to a minimum. But there still remained certain possible canses of variation. Since mangels are easily cross-fertilised, a commercial strain will not be a pure one, and so varying parentage may be a possible canse of variation in constitution. The slight differences in soil will vary the food supply; the hoeing will not be absolutely regular; the distribution of manure will not be perfectly uniform, and slight errors may enter into the analyses of the roots. Each of these separate causes is equally likely to make the percentage of dry matter in an individual root higher or lower than the average value. In the majority of cases, some of these causes will tend to raise, and some to lower the result. It will only happen in relatively few cases that all the causes of variation act in the same direction, yielding a result differing considerably from the mean value. A curve showing the frequencies of different percentages of dry matter should thus have its maximum at the mean value of the percentage, and should be symmetrical about the mean value. It might, in fact, be expected to yield a curve of the same general form as the error curve shown in figure 3.

The actual distribution of frequencies is shown in figure 5 . In this diagram, the percentage of dry matter is represented along the horizontal axis, and the number of roots along the vertical axis. For each root a dot is placed in the diagram above the corresponding percentage. The whole range of variation is divided into intervals, from $10-11 \%, 11-12 \%$, etc., and an ordinate is erected at the middle point of each section, of a length proportional to the number of dots in that section. The tops of these ordinates are represented in the diagram by crosses. It is clear from inspection that it would be possible to draw a smooth symmetrical curve passing very near to the tops of the ordinates. The form of such a curve would be in good agreement with that of the ideal error curve shown in figure 3. To test this more closely, the Gauss error curve was drawn for comparison with the actual distribution. This curve is the smooth curve

* Wood and Stratton, "The Interpretation of Experimental Results," Journal of Agricultural Science, Vol. III, part 4. 
shown in figure 5. The method of construction of this curve will be explained later on, but meanwhile we may note that the crosses representing the actual distribution all lie very near to the curve.

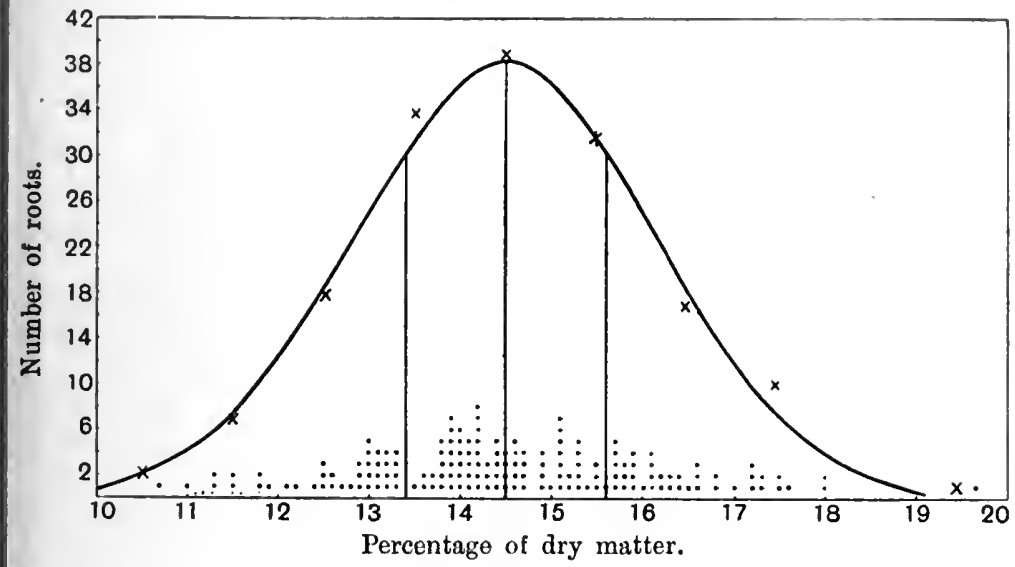

Fig. 5 .

The P.E. of a single determination is given as $1 \cdot 1 \%$. Thus the P.E. of the A.M. is $\frac{1 \cdot 1}{\sqrt{160}}=0.09$. In the diagram, ordinates are drawn on each side of the mean, distant $1 \cdot 1$ from it. According to the theoretical discussion, 80 of the observed values should lie within, and 80 outside these limits. A count of the dots shows that 81 observations are within $1.1 \%$ of the mean, and 79 outside these limits - a result in excellent agreement with the demands of the theory.

The equation of the normal error curve is

$$
y=\frac{h}{\sqrt{ } \pi} e^{-h^{2} x^{2}},
$$

where

$$
h=\frac{\cdot 47696}{r}=\frac{\cdot 47696}{1 \cdot 1}=\cdot 43 .
$$

A sufficient number of ordinates to enable us to draw the curve can easily be evaluated by means of a table of logarithms.

In the preceding example, the symmetry of the distribution of frequencies about the A.s. appears to justify the assumption that the different causes of variation in content of individual roots were equally likely to raise as to lower the result. In general, when some of the causes of variation are dissymmetrical in their action, tending to give high results oftener than low results, or vice versa, the frequency curve is unsymmetrical, even when the 
number of observations is sufficiently large to give the laws of chance fair play. If the number of observations be large, and the curve unsymmetrical, it is generally safe to assume that there is at work some cause which tends to act always in one direction, giving an abnormal number of high or of low results. Such a case is discussed in Example 4 below.

Example 4. The average weight of a number of mangel roots*.

The figure shows the distribution of the weights of 196 roots. In this case no smooth curve has been drawn, but the tops of successive ordinates have been joined by straight lines. The resulting curve is clearly dissymmetrical, showing that either an abnormally large number of large roots, or an abnormally small number of small roots, was taken. The latter alternative probably affords the true explanation, as the weak plants which would produce small roots would be destroyed in the process of hoeing and singling;

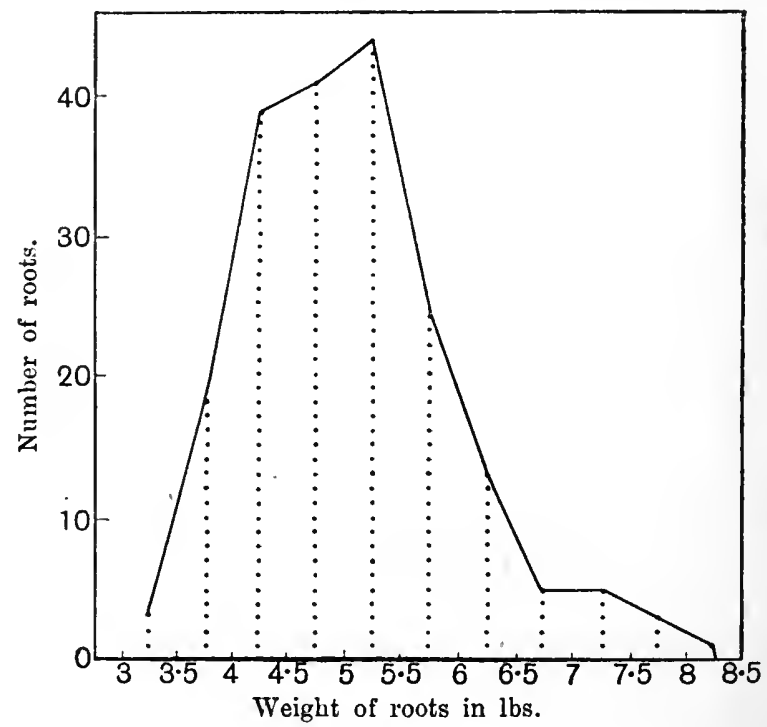

Fig. 6.

and it is possible that the very small roots would be unconsciously passed over in sampling. The true curve of frequency should therefore extend further in the direction of small weights. The effect of the absence of small roots is to give an apparent mean weight greater than the true value. The method of least squares is not strictly applicable to such a distribution as is here shown, as the theory demands a distribution of frequencies which shall be symmetrical about the mean.

* Wood and Stratton, loc. cit. 
Example 5. Magnitude-interval of a parallel-wire grating*.

A parallel-wire grating consists of a series of equidistant parallel wires fitted on a frame. When this is set over the object glass of a telescope, it acts as a diffraction-grating, so that each star, instend of producing a single dot upon the photographic plate, produces a central bright dot, with a series of dots of decreasing lrightness on each side of it. The dots are in reality small spectra, but on account of the smallness of thedispersion they are sensibly round. The proportion of the light of a star which is deviated into an image of a given order is constant, depending only on the form of the grating. There is accordingly a definite magnitude-interval between a central image and the auxiliary image on each side of it. There will be this same magnitudeinterval between a given star and another star whose central image is equal in size and greyness to the first diffraction image of the first star. Thus when the magnitude-interval between consecutive diffraction images is known, the magnitudes of all the stars on the plate can be determined, provided there are on the plate some stars whose magnitudes are known. The adjoining table shows a series of determinations of the magnitude-interval of a grating, based on measurements of a number of stars upon plates with different exposures.

\begin{tabular}{|c|c|c|c|c|c|c|c|c|c|c|}
\hline No. of plate... & 4996 & 5014 & 5061 & 4922 & 4923 & 4924 & 4997 & 5059 & 5070 & 5023 \\
\hline \multicolumn{11}{|l|}{ Maguitude } \\
\hline $8 \cdot 89$ & $2 \cdot 68$ & $2 \cdot 66$ & 3.09 & $2 \cdot 66$ & $2 \cdot 73$ & $2 \cdot 31$ & $2 \cdot 65$ & $2 \cdot 67$ & $2 \cdot 94$ & - \\
\hline 10.42 & $2 \cdot 67$ & $2 \cdot 90$ & $2 \cdot 62$ & $2 \cdot 67$ & $2 \cdot 75$ & $2 \cdot 86$ & 2.9 .8 & $2 \cdot 60$ & $2 \cdot 68$ & $2 \cdot 57$ \\
\hline 10.54 & $2 \cdot 74$ & $2 \cdot 86$ & $2 \cdot 84$ & $2 \cdot 68$ & $2 \cdot 66$ & - & $2 \cdot 67$ & $2 \cdot 46$ & $2 \cdot 60$ & $2 \cdot 75$ \\
\hline $10 \cdot 62$ & $2 \cdot 56$ & $2 \cdot 54$ & $2 \cdot 64$ & $2 \cdot 78$ & $2 \cdot 62$ & $2 \cdot 69$ & $2 \cdot 72$ & $2 \cdot 65$ & $2 \cdot 59$ & - \\
\hline $10 \cdot 64$ & $2 \cdot 49$ & $2 \cdot 58$ & $2 \cdot 77$ & $2 \cdot 70$ & $2 \cdot 70$ & $2 \cdot 77$ & $2 \cdot 66$ & $2 \cdot 72$ & $2 \cdot 50$ & - \\
\hline $10 \cdot 64$ & $2 \cdot 56$ & $2 \cdot 69$ & $2 \cdot 62$ & $2 \cdot 70$ & $2 \cdot 70$ & $2 \cdot 73$ & $2 \cdot 66$ & $2 \cdot 61$ & $2 \cdot 46$ & - \\
\hline $10 \cdot 66$ & - & - & - & $2 \cdot 76$ & $2 \cdot 63$ & $2 \cdot 64$ & $\ldots$ & - & - & - \\
\hline $10 \cdot 69$ & - & - & $2 \cdot 72$ & $2 \cdot 74$ & $2 \cdot f i 6$ & $2 \cdot 94$ & 一 & - & $2 \cdot 82$ & - \\
\hline $10 \cdot 90$ & $2 \cdot 78$ & $2 \cdot 73$ & $2 \cdot 91$ & $2 \cdot 64$ & $3 \cdot 03$ & - & - & - & - & - \\
\hline 10.94 & $2 \cdot 82$ & - & $2 \cdot 85$ & - & - & - & - & - & - & - \\
\hline $10 \cdot 95$ & $2 \cdot 79$ & $2 \cdot 95$ & $2 \cdot 77$ & $2 \cdot 60$ & $2 \cdot 59$ & $2 \cdot 99$ & - & - & - & - \\
\hline $11 \cdot 01$ & - & $2 \cdot 69$ & $2 \cdot 89$ & - & - & - & - & - & - & - \\
\hline $11 \cdot 03$ & $2 \cdot 76$ & $2 \cdot 79$ & $2 \cdot 62$ & $2 \cdot 55$ & $2 \cdot 97$ & - & - & - & - & 一 \\
\hline $11 \cdot 12$ & $2 \cdot 65$ & - & $2 \cdot 80$ & $2 \cdot 41$ & $2 \cdot 87$ & $2 \cdot 86$ & - & 一 & - & -. \\
\hline $11 \cdot 26$ & $2 \cdot 67$ & - & $2 \cdot 77$ & $2 \cdot 32$ & $2 \cdot 87$ & - & - & 一 & 一 & 一 \\
\hline $11 \cdot 47$ & 3.00 & - & - & - & - & - & - & 一 & -- & - \\
\hline 11.57 & $2 \cdot 68$ & - & - & - & - & - & - & - & - & - \\
\hline 11.97 & $2 \cdot 63$ & - & 一 & - & - & - & - & 一 & - & - \\
\hline $12 \cdot 12$ & $2 \cdot 54$ & - & - & $\longrightarrow$ & - & - & - & - & - & - \\
\hline $12 \cdot 25$ & $2 \cdot 83$ & - & 一 & - & - & - & - & - & - & - \\
\hline $12 \cdot 32$ & 279 & - & - & - & - & - & - & - & - & - \\
\hline
\end{tabular}

* Chapman and Melotte, Monthly Noticer, R.A.S., Norember, 1913. 
The mean of 98 determinations $=2^{\mathrm{m}} \cdot 71$.

The P. E. of a single estimate calculated from the squares of the residuals

$$
= \pm 0^{\mathrm{m} \cdot 097} \text {. }
$$

The P. E. of the mean value is $\frac{\cdot 097}{\sqrt{98}}=\cdot 0097$.

The final result may thus be written

$$
2^{\mathrm{m}} \cdot 71 \pm \cdot 0097 .
$$

The P.E. of a single estimate calculated from the sum of the residuals is 094 .

The frequency curve is shown in figure 7. It is seen that the A.M. does not coincide with the maximum ordinate. The latter occurs at about $2^{\mathrm{m} \cdot 67}$, whereas the A.M. is $2^{\mathrm{m} \cdot 71}$. The number of measurements represented by the

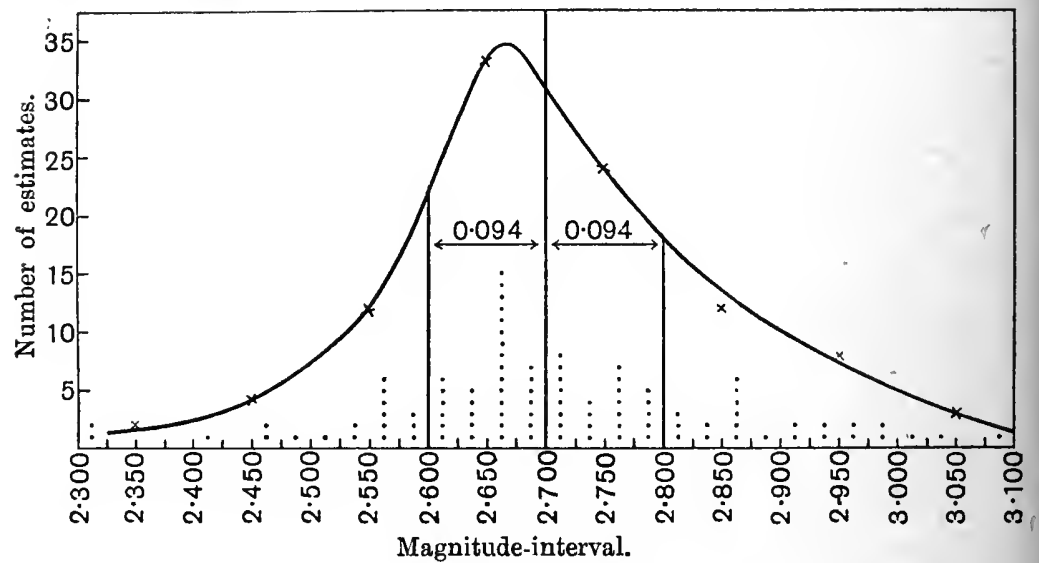

Fig. 7.

curve is 98 , and this appears to be a sufficiently large number to yield an accurate representation of the true nature of the curve of errors. We are forced to conclude that there is present some cause of dissymmetry.

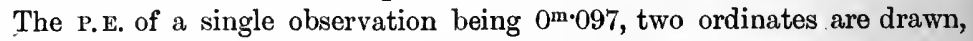
one on each side of the A.M., and at a distance of $0^{\mathrm{m} \cdot 097}$ from it. It is found that 57 measurements lie between these limits, and 41 outside them. Theory would require 49 observations to lie within the limits $\pm 0^{\mathrm{m} \cdot 097}$, and 49 outside these limits. It is thus no longer strictly possible to attach the original meaning to the P.E. deduced from the residuals on the assumption that the mean is the true value of the unknown.

When the curve of frequencies is of the form of the curve in figure 7 , indicating a genuine dissymmetry in the distribution of frequencies, it would perhaps be better to adopt the abscissa corresponding to the maximum frequency, as the most plausible value of the unknown. The value of the 
P. E. deduced from the fornula may be taken to indicate roughly whether the observations are closely clustered about the mean, or are spread over a considerable range. But this $P$.E. cannot be regarded as having the meaning originally attached to the $\mathrm{P}$.E., since the eurve representing the frequency distribution is not of the form of the Gauss error curve represented in figure 3.

\section{Example 6. Glume-length of wheat.}

Figure 8 shows the results of measuring the length of the glumes of 595 individual wheat plants. The eurve shows three well-defined maxima, and this faet in itself would arouse suspicion as to the purity of strain of the wheat measured. The plants measured were, as a matter of fact, the second generation from a cross between Rivet wheat (with an average glume-

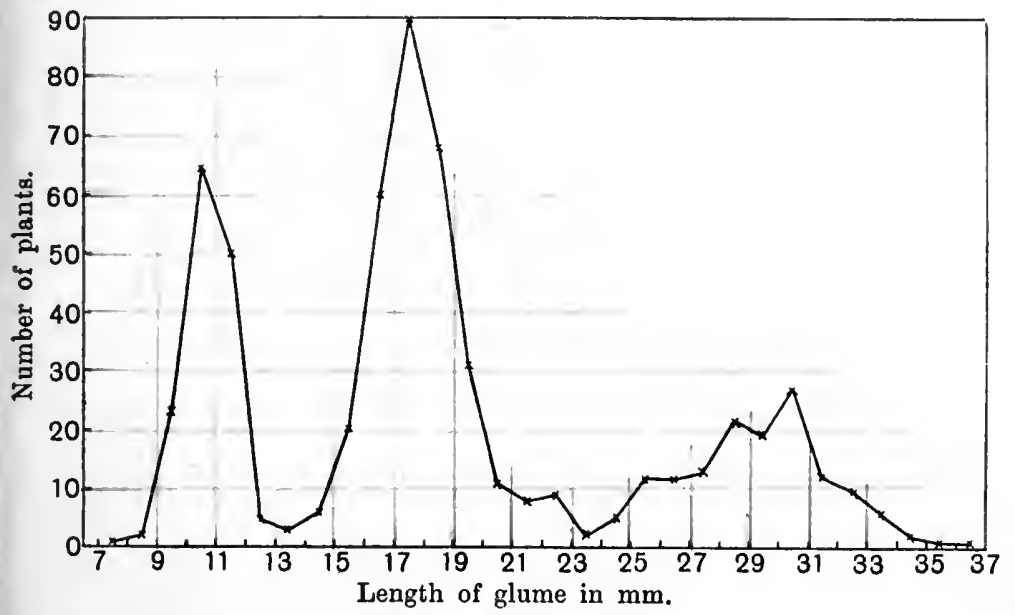

Fig. 8.

length of $9 \mathrm{~mm}$.) and Polish wheat (with an average glume-length of $28 \mathrm{~mm}$.). The curve shows that the plants examined are divided into three well-defined groups, one resembling the short-glumed Rivet parent, one resembling the long-glumed Polish parent, and the third intermediate between these two with an a verage glume-length of $17 \mathrm{~mm}$.

The application of the urdinary least-square method to such material as is here represented is quite meaningless. This particular example illustrates very clearly the utility of commencing the discussion of a number of obserrations by drawing the eurve of frequencies. Here the non-homogeneous nature of the material is immediately shown by the curve: while in cases like those considered in Examples 4 and 5 above, the curvo of frequencies shows the presence of a systematic error. 


\section{EXAMPLES.}

In the following Examples ealculate the P. E. from both Gauss's and Peters' formula.

1. Evaluate the P.E. of a single determination, and of the mean value, of the variable tabulated on page 6 .

2. The following table gives 12 determinations of the azimuth of Allen from Sears, Texas. (U.S. C. and G. Survey. Publications, No. 14, p. 149.)

$98^{\circ} \quad 6^{\prime} \quad 41^{\prime \prime} \cdot 5$
$42^{\prime \prime} \cdot 8$
$43^{\prime \prime} \cdot 4$
$43^{\prime \prime} \cdot 1$
$39^{\prime \prime} \cdot 7$
$42^{\prime \prime} \cdot 7$
$41^{\prime \prime} \cdot 6$
$43^{\prime \prime} \cdot 3$
$40^{\prime \prime} \cdot 0$
$45^{\prime \prime} \cdot 0$
$43^{\prime \prime} \cdot 3$
$40^{\prime \prime} \cdot 7$

Find the mean value, the P. $\mathrm{E}$. of a single determination, and the P.E. of the mean.

3. From the following $\mathbf{1 5}$ independent determinations of the coefficient of expansion of dry air (Rudberg, Poggendorff's Annalen, 41, p. 271), find the A.X. and its P.E.:

$\begin{array}{ccl}3 \cdot 643 \times 10^{-3} & 3 \cdot 636 \times 10^{-3} & 3 \cdot 646 \times 10^{-3} \\ 54 & 51 & 3 \cdot 662 \\ 44 & 43 & 3 \cdot 840 \\ 50 & 43 & 3.902 \\ 53 & 45 & 3 \cdot 652\end{array}$

25. Probable error of any function of a number of independent quantities whose probable errors are known.

Let $m_{1}, m_{2}, \ldots, m_{n}$ be $n$ independent quantities, whose P.E.'s are $r_{1}, r_{2}, \ldots, r_{n}$, and let

$$
F=f\left(m_{1}, m_{2}, \ldots, m_{n}\right)
$$

be the function of $m_{1}, m_{2}$, etc. whose P.E. is required.

If $d F$ be the error in the value of $F$ produced by errors $d m_{1}, d m_{2}$, etc. in the values of $m_{1}, m_{2}$, etc., then

$$
\begin{aligned}
F+d F & =f\left(m_{1}+d m_{1}, m_{2}+d m_{2}, \ldots, m_{n}+d m_{n}\right) \\
& =f+\frac{\partial f}{\partial m_{1}} d m_{1}+\frac{\partial f}{\partial m_{2}} d m_{2}+\ldots+\frac{\partial f}{\partial m_{n}} d m_{n} .
\end{aligned}
$$


The error in $F$ is thus given by

where

$$
\begin{gathered}
d F=\frac{\partial f}{\partial m_{1}} d m_{1}+\frac{\partial f}{\partial m_{2}} d m_{2}+\ldots+\frac{\partial f}{\partial m_{n}} d m_{n} \\
=a_{1} d m_{1}+a_{2} d m_{2}+\ldots+a_{n} d m_{n}, \\
a_{1}=\frac{\partial f}{\partial m_{1}}, \quad a_{2}=\frac{\partial f}{\partial m_{2}}, \text { etc. }
\end{gathered}
$$

From this point onward the problem is reduced to that of finding the P.E. of a linear function of $n$ independent variables.

The result may be written

or

$$
\begin{aligned}
r^{2} & =a_{1}{ }^{2} r_{2}{ }^{2}+a_{2}{ }^{2} r_{2}^{2}+\ldots, \text { etc. } \\
\mu^{2} & =a_{1}^{2} \mu_{1}^{2}+a_{2}^{2} \mu_{2}{ }^{2}+\ldots, \text { etc. }
\end{aligned}
$$

where

$$
a_{8}=\frac{\partial f}{\partial m_{8}} \text {. }
$$

The above formulae sometimes break down in practice, when the errors are not small enough to justify the neglect of the square and higher terms in the Taylor expansion.

\section{EXAMPLES.}

1. If $x$ and $y$ be the sides of a rectangle, and $r_{1}, r_{2}$ their P.E.'s, find the P.E. $r$ of the area of the rectangle.

Let

Then

$$
\begin{gathered}
z=x y . \\
d z=y d x+x d y .
\end{gathered}
$$

- Applying the formula derived above, we find

$$
r^{2}=y^{2} r_{1}^{2}+x^{2} r_{2}{ }^{2}
$$

2. The edge of a cube is of length $a$, and its P.E. is $r$. Find the P.E. of the volume of the cube.

Ans. $3 a^{2} r$.

3. The P.E.'s of the three edges of a cube are $r_{1}, r_{2}, r_{3}$. Find the P.E. of the volume. Ans. $\quad a^{2}\left(r_{1}^{2}+r_{2}^{2}+r_{3}^{2}\right)^{\frac{1}{2}}$.

4. Given that the P.E. of $x$ is $r$, find the P.E.'s of $e^{-x}, \log x, \cos x$, and $(4+x)^{3}$.

5. If the P.E. of a single reading of the graduated circle on a meridian circle be $r$, what is the P.E. of a result based on the readings of $n$ microscopes placed at $n$ different points of the circle?

Ans. $\frac{r}{\sqrt{n}}$.

6. Given the following telegraphic longitude determinations, find the longitude of Moscow east of Greenwich, and estimate its P.E.

$\begin{array}{llllcr}\text { Potsdam-Greenwich } & \ldots & \ldots & 0^{\mathrm{h}} & 52^{\mathrm{m}} & 16^{8} \cdot 051 \pm 0^{8} \cdot 003 . \\ \text { Pulkowa-Potsdam } & \ldots & \ldots & 1 & 9 & 2 \cdot 491 \pm 0 \cdot 0 \mathrm{C} 3 . \\ \text { Moscow-Pulkowa } & \ldots & \ldots & 0 & 28 & 58 \cdot 450 \pm 0 \cdot 010 .\end{array}$

B. 0 . 
7. Given the sides $a, b$, and the angle $C$ of a triangle, and their P.E.'s, find the P.E.'s of the side $c$, and of the area of the triangle.

$$
\begin{aligned}
c^{2} & =a^{2}+b^{2}-2 a b \cos C, \\
c d c & =(a-b \cos C) d a+(b-a \cos C) d b+a b \sin C d C \\
& =c \cos B d a+c \cos A d b+a b \sin C d C ; \\
\therefore \quad d c & =\cos B d a+\cos A d b+a \sin B d C .
\end{aligned}
$$

Applying the formula derived above, we find

$$
r_{c}^{2}=r_{a}^{2} \cos ^{2} B+r_{b}^{2} \cos ^{2} A+r_{c}^{2} a^{2} \sin ^{2} B \sin ^{2} l^{\prime \prime},
$$

where $r_{C}$ is measured in seconds of arc.

$$
\begin{aligned}
\Delta & =\frac{1}{2} a b \sin C, \\
\frac{d \Delta}{\Delta} & =\frac{d a}{a}+\frac{d b}{b}+\cot C d C, \\
\frac{r_{\Delta}^{2}}{\Delta^{2}} & =\frac{r_{a}^{2}}{a^{2}}+\frac{r_{b}^{2}}{b^{2}}+\cot ^{2} C \sin ^{2} 1^{\prime \prime} r_{C}^{2} .
\end{aligned}
$$

8. The coefficient of expansion of a rod is determined by measuring its length at two different temperatures. If the length be $l_{1}$ at a temperature $t_{1}$, and $l_{2}$ at a higher temperature $t_{2}$, the coefficient is given by

$$
a=\frac{l_{2}-l_{1}}{l_{1}\left(t_{2}-t_{1}\right)} .
$$

If $r_{t}$ be the P.E. of a temperature-reading, and $r_{l}$ the P.E. of a lengthdetermination, find the P.E. of $a$.

The P.E. of $l_{2}-l_{1}$ is $\sqrt{2} r_{l}$, and P.E. of $t_{2}-t_{1}, \sqrt{2} r_{t}$. Taking logarithms of both sides of the equation for $a$, and differentiating, we obtain

whence we find

$$
\frac{d a}{a}=\frac{d\left(l_{2}-l_{1}\right)}{l_{2}-l_{1}}-\frac{d l_{1}}{l_{1}}+\frac{d\left(t_{2}-t_{1}\right)}{t_{2}-t_{1}},
$$

$$
\begin{aligned}
\frac{r_{a}^{2}}{a^{2}} & =\frac{2 r_{l}^{2}}{\left(l_{2}-l_{1}\right)^{2}}+\frac{r_{l}^{2}}{l_{1}^{2}}+\frac{2 r_{t}^{2}}{\left(t_{2}-t_{1}\right)^{2}} \\
& =r_{l}^{2}\left(\frac{1}{l_{1}^{2}}+\frac{2}{\left(l_{2}-l_{1}\right)^{2}}\right)+\frac{2 r_{t}^{2}}{\left(t_{2}-t_{1}\right)^{2}} .
\end{aligned}
$$

\section{Errors due to separable causes.}

When the accidental errors which enter into a measurement can be divided into a number of separate parts, each of which is independent of the others, and has a known M.S.E. or P.E., the M.S.E. or P.E. of the whole error can be subdivided into the same number of parts. For if an error $\epsilon$ be composed of three separate parts $\epsilon_{1}, \epsilon_{2}, \epsilon_{3}$, all independent of one another, we have

$$
\epsilon=\epsilon_{1}+\epsilon_{2}+\epsilon_{3} \text {. }
$$


Then it follows as in $\$ 22$ that

and

$$
\begin{gathered}
\mu^{2}=\mu_{1}^{2}+\mu_{2}^{2}+\mu_{3}^{2}, \\
r^{2}=r_{3}{ }^{2}+r_{2}{ }^{2}+r_{3}{ }^{2} .
\end{gathered}
$$

\section{EXAMPLE.}

A base line is measured by successive end to end placings of a rod, and is found to be 100 times the length of the rod. If $a$ be the P.E. of the assumed length of the rod, due to uncertainty of the assumed temperature, and $b$ be the P.E. of the end to end placings of the rod, and $c$ the P.E. of the settings at the extremities of the base line, find the P.E. of the assumed length of the base.

(1) If the error in the assumed length of the rod be $x$, the resulting error in the length of the base will be $100 x$. The P.E. of the length of the base, having regard to this class of error only, will be $100 a$.

(2) There will be 100 settings of the rod, and the P.E. which enters at each of the 99 intermediate points will be $b$. The P.E. of the length of the base, if this be the only kind of error, will be $\sqrt{99} b$.

(3) The P.E. of the length of the base, due to the end readings, will be $\sqrt{2} c$

Thus, if $r$ be the total P.E. of the length of the base,

$$
r^{2}=100^{2} a^{2}+99 b^{2}+2 c^{2} \text {. }
$$

\section{Total Probable Error when a Systematic Error is} present.

The case where the total error in an observation is partly due to accidental causes, and partly due to systematic causes, follows naturally from the discussion of the last paragraph.

Let $r=$ P.E. of an observation, when the accidental errors only are considered,

$r_{0}=$ the mean error arising from the systematic causes, $r^{\prime}=$ total P.E. of such an observation.

Then

$$
r^{\prime 2}=r^{2}+r_{0}^{2}
$$

as in $§ 22$.

If the observation be repeated $n$ times, and the mean taken the final P.E. $r^{\prime \prime}$ is given by

$$
r^{\prime \prime 2}=\frac{r^{2}}{n}+r_{0}^{2}
$$


The systematic error is in no way affected by the repetition of the observation, while the P.E. due to the accidental causes is decreased to $\frac{r}{\sqrt{n}}$.

The correct interpretation of the last equation is of vital importance in all applications of our present subject. If only accidental errors are present, and if $r$ be the P.E. of a single observation, then the P.E. of the mean of $n$ observations is $\frac{r}{\sqrt{n}}$. This expression would lead one to expect that it would be possible to obtain a result free from error simply by increasing the number of observations. In practice, however, it is impossible to eliminate all traces of systematic errors. If the systematic error be represented by $r_{0}$, we have seen above that the total P.E. of the mean of $n$ observations is $\sqrt{\frac{r^{2}}{n}+r_{0}^{2}}$. It may happen that when $n$ is relatively small $r_{0}^{2}$ is negligible in comparison with $\frac{r^{2}}{n}$; but so long as $r_{0}$ is finite, however small it may be, by increasing the value of $n$ we shall eventually reach a stage where $\frac{r^{2}}{n}$ becomes negligible in comparison with $r_{0}^{2}$. When such a stage has been reached, no further improvement in the precision of the mean value can be attained by increasing the number of observations. Or, to put this in other words, as the number of observations is made greater and greater, a stage is eventually reached beyond which the error of the result is fixed by the constant or systematic errors, and not by the probable accidental error. A further increase in the number of observations produces no corresponding increase in the accuracy of the result, unless the conditions of observation can be varied from time to time, so as to vary the magnitude and sign of the systematic errors, thus causing them to appear effectively as accidental errors.

The P.E. computed from the residuals is independent of the presence of a constant error. For if a constant quantity be added to all the observed values in a series, the mean value is altered by the same amount, and the residuals (and the P.E.) remain unchanged. The probable error is a measure, not of the deviations 
of the observed values from the true value, but of their deviations from the mean of an infinite number of observations.

28. The Correction of Statistics for the Effects of a known Probable Error of Observation.

When a table is construeted to exhibit the distribution of frequencies of successive values of a certain measured property whose P.E. is known, it is necessary to eonsider the effect of the probable error upon the table. For simplieity's sake we shall consider in detail the problem discussed by Eddington* in his treatment of this problem.

Suppose we have a table giving the results of the counts of stars between given limits of magnitude, and suppose the P.E. of a magnitude determination is known. Let this P.E. be $0.477 \frac{1}{h}$, so that the probability of an error $x$ is $C e^{-h^{2} x^{2}}$.

Let $u(m) d m=$ observed number of stars between magnitudes $m$ and $m+d m$,

$v(m) d m=$ true number.

Of the stars whose true magnitude is between $(m+x)$ and $(m+x+d x)$ the proportion $\frac{h}{\sqrt{\pi}} e^{-h^{2} x^{2}} d x$ will have an error between $-x$ and $-(x+d x)$, and will be observed as of magnitude $m$. Thus we have

$$
u(m)=\frac{h}{\sqrt{\pi}} \int_{-\infty}^{+\infty} v(m+x) e^{-h^{2} x^{2}} d x .
$$

By the symbolic form of Taylor's theorem

$$
v(m+x)=e^{x \frac{d}{d m}} v(m),
$$

and therefore

$$
u(m)=\frac{h}{\sqrt{\pi}} \int_{-\infty}^{+\infty} e^{x \frac{d}{d m}-x^{2} h^{2}} v(m) d x .
$$

Treating this integral as a special case of

$$
\int_{-\infty}^{+\infty} e^{-a_{1} x-a_{2} x^{2}} d x=\sqrt{\frac{\pi}{a_{2}}} \exp \frac{a_{1}^{2}}{4 a_{2}},
$$

* Monthly Notices, R.A.S., Vol. Lxxru, pp. 3559, 360, from which the whole of this discussion has been tảken. 
we find

$$
u(m)=\exp \left(\frac{1}{4 h^{2}} \frac{d^{2}}{d m^{2}}\right) v(m)
$$

and

$$
\begin{aligned}
v(m) & =\exp -\left(\frac{1}{4 h^{2}} \frac{d^{2}}{d m^{2}}\right) u(m) \\
& =u(m)-\frac{1}{4 h^{2}} u^{\prime \prime} m+\frac{1}{2 !}\left(\frac{1}{4 h^{2}}\right)^{2} u^{\mathrm{iv}}(m)-\text { etc. }
\end{aligned}
$$

When the P.E. is small, it is sufficient to consider the first and second terms only. If $\alpha$ be the successive intervals of magnitude, the tabular second difference is

For

$$
u(m+\alpha)+u(m-\alpha)-2 u(m)=\alpha^{2} u^{\prime \prime}(m) \text { approximately. }
$$

$$
u(m+\alpha)=u(m)+\alpha u^{\prime}(m)+\frac{\alpha^{2}}{2} u^{\prime \prime}(m) \text { approximately, }
$$

and

$$
\begin{aligned}
u(m-\alpha) & =u(m)-\alpha u^{\prime}(m)+\frac{\alpha^{2}}{2} u^{\prime \prime}(m) \quad ” \\
\frac{1}{2 h} & =1.046 \times \text { probable error. }
\end{aligned}
$$

Thus the approximate correction is

- $\left(\frac{\mathbf{1} \cdot 046 \times \text { probable error }}{\text { tabular interval }}\right)^{2} \times$ tabular second difference.

\section{The Precision of the Probable Error deduced} from $r=0.6745$

In $\S 23$ we derived certain expressions for the residuals of $n$ observations in terms of the true errors of the observations. If each of these expressions be squared, and the results added together, we obtain the expression

$$
[v v]=\frac{1}{n^{2}}\left\{n(n-1)[\epsilon \epsilon]-2 n\left[\epsilon_{8} \epsilon_{t}\right]\right\},
$$

where $\left[\epsilon_{s} \epsilon_{t}\right]$ denotes the sum of the products of all possible pairs from among the $n$ quantities $\epsilon_{1}, \epsilon_{2}, \ldots, \epsilon_{n}$.

The equation may be written

$$
\frac{[v v]}{n-1}=\frac{[\epsilon \epsilon]}{\dot{n}}-\frac{2}{n(n-1)}\left[\epsilon_{s} \epsilon_{t}\right] \text {. }
$$

If we formed the equation for a large number of samples 
of $n$ observations from among a very large number of observations, and took the average value of each side of the equation, we should obtain

$$
\frac{[v v]}{n-1}=\mu^{2}-\text { mean value of } \epsilon_{8} \epsilon_{t} \text {. }
$$

The derivation of the usual formula for $\mu^{2}$ seems therefore to be equivalent to regarding the mean value of $\epsilon_{g} \epsilon_{t}$ as zero. That this is justifiable can easily be seen from $\$ 20$, equation $\left(4^{\prime}\right)$, where it was found that the mean value of the product $V_{s} V_{t}$, for all possible products among $N$ residuals, is equal to $-\frac{\mu^{2}}{N-1}$. When the number of observations $N$ is sufficiently large, it was seen that the $V$ 's could be regarded as the true errors $\epsilon$, so that we may say that

$$
\text { the mean value of } \epsilon_{8} \epsilon_{t}=-\frac{\mu^{2}}{N-1} \text {. }
$$

When the number of observations $N$ is very large, this quantity becomes vanishingly small, and may be negleeted. Our equation above may then be written

$$
\frac{[v v]}{n-1}=\mu^{2}
$$

This equation yields the mean result for a large number of cases. But in an individual set of $n$ observations it will not be strictly true, and $\sqrt{\frac{[v v]}{n-1}}$ will not be the accurate M.S.E. of an infinite series of observations of which the given $n$ observations form a random sample.

The error made in estimating $\mu^{2}$ from the residuals in any particular case is

$$
\frac{[v v]}{n-1}-\mu^{2}=\frac{[\epsilon \epsilon]}{n}-\frac{2}{n(n-1)}\left[\epsilon_{s} \epsilon_{t}\right]-\mu^{2} .
$$

The square of this error is

$$
\begin{aligned}
\frac{[\epsilon \epsilon]^{2}}{n^{2}}+\frac{4}{n^{2}(n-1)^{2}}\left[\epsilon_{8} \epsilon_{t}\right]^{2}- & \frac{4}{n^{2}(n-1)}[\epsilon \epsilon]\left[\epsilon_{8} \epsilon_{t}\right]+\mu^{4} \\
& -2 \frac{[\epsilon \epsilon]}{n} \mu^{2}+\frac{4}{n(n-1)}\left[\epsilon_{8} \epsilon_{t}\right] \mu^{2} .
\end{aligned}
$$

If we take the mean value of this expression for a large 
number of cases, the result will be the (M.S.E.) $)^{2}$ of $\mu^{2}$. The mean values of all the terms in the expression above will be considered in turn :

and

$$
[\epsilon \epsilon]^{\prime \prime}=\left[\epsilon^{4}\right]+2\left[\epsilon_{s}^{2} \epsilon_{t}^{2}\right]
$$

$$
\begin{gathered}
{\left[\epsilon^{4}\right]=n \times \text { mean value of } \epsilon^{4}=\frac{2 n h}{\sqrt{\pi}} \int_{0}^{\infty} x^{4} e^{-h^{2} x^{2}} d x=\frac{3 n}{4 h^{4}}=3 n \mu^{4},} \\
\begin{aligned}
2\left[\epsilon_{s}{ }^{2} \epsilon_{t}{ }^{2}\right] & =2 \times \frac{n(n-1)}{2} \times \text { mean value of } \epsilon_{s}{ }^{2} \epsilon_{t}{ }^{2} \\
= & n(n-1) \mu^{4} ;
\end{aligned}
\end{gathered}
$$

therefore

$$
\text { mean value of } \frac{[\epsilon \epsilon]^{2}}{n^{2}}=\frac{3 \mu^{4}}{n}+\frac{n-1}{n} \mu^{4} \text {. }
$$

Again

$$
\left[\epsilon_{8} \epsilon_{t}\right]^{2}=\left[\epsilon_{8}{ }^{2} \epsilon_{t}^{2}\right]+\text { terms involving first powers of } \epsilon \text {. }
$$

When the mean value is formed the terms involving first powers vanish, therefore

$$
\begin{aligned}
& \text { mean value of } \frac{4\left[\epsilon_{\delta} \epsilon_{t}\right]^{2}}{n^{2}(n-1)^{2}} \\
& \quad=\frac{4}{n^{2}(n-1)^{2}} \times \frac{n(n-1)}{2} \times \text { mean value of } \epsilon_{s}{ }^{2} \epsilon_{t}{ }^{2} \\
& =\frac{2}{n(n-1)} \mu^{4} .
\end{aligned}
$$

The mean value of the third term vanishes on account of the factor $\left[\epsilon_{8} \epsilon_{t}\right]$. The mean value of the fifth term is $-2 \mu^{4}$, and the sixth term vanishes on account of the factor $\left[\epsilon_{\delta} \epsilon_{t}\right]$.

Thus we obtain the equations

$$
\begin{aligned}
\text { (M.S.E.) })^{2} \text { of } \mu^{2} & =\frac{3 \mu^{4}}{n}+\frac{n-1}{n} \mu^{4}+\frac{2}{n(n-1)} \mu^{4}-\mu^{4} \\
& =\frac{2 \mu^{4}}{n-1}, \\
\text { M.S.E. of } \mu^{2} & =\mu^{2} \sqrt{\frac{2}{n-1}} .
\end{aligned}
$$

Since $\quad$ M.S.E. of $\mu^{2}=2 \mu \times$ M.S.E. of $\mu$, we finally obtain the result

$$
\frac{\text { M.S.E. of } \mu}{\mu}=\frac{1}{\sqrt{2(n-1)}}=\frac{\cdot 707}{\sqrt{n-1}} .
$$


Also, since $r=0.6745 \mu$, we have the relations

and

$$
\frac{\text { M.S.E. of } r}{r}=\frac{\cdot 707}{\sqrt{n-1}}
$$$$
\text { P.E. of } r=\frac{-4769}{r}=
$$

where $r$ is derived from the formula $r=0.6745 \sqrt{n-1} \begin{gathered}{[v v]} \\ n-\end{gathered}$.

The following table gives the factor $\frac{4769}{\sqrt{n-1}}$ for some different values of $n$.

\begin{tabular}{|r|c|c|l|}
\hline$n$ & $\frac{\text { P.e. of } r}{r}$ & $20 \%$ error & $50 \%$ error \\
\cline { 1 - 2 } 5 & $\cdot 288$ & $\cdot 64$ & $\cdot 24$ \\
10 & $\cdot 159$ & $\cdot 40$ & $\cdot 034$ \\
15 & $\cdot 127$ & $\cdot 29$ & $\cdot 008$ \\
20 & $\cdot 109$ & $\cdot 21$ & $\cdot 0002$ \\
30 & $\cdot 089$ & $\cdot 12$ & $\cdot 00014$ \\
40 & $\cdot 076$ & $\cdot 076$ & $8 \times 10^{-6}$ \\
50 & $\cdot 068$ & $\cdot 047$ & $6 \times 10^{-7}$ \\
60 & $\cdot 062$ & $\cdot 030$ & $5 \times 10^{-8}$ \\
100 & $\cdot 048$ & $\cdot 0050$ & \\
\hline
\end{tabular}

By the aid of this table, and the table of $\Theta(t)$ given in Appendix I, we may gain some idea of the number of observations which must be taken in order to yield values of the P.E. which are deserving of confidence. In the third and fourth columns above are given the probability that the P.E. should be $20 \%$ out, and $50 \%$ out, respectively. It is seen that with 10 observations the odds are only 3 to 2 that the calculated P.E. is within $20 \%$ of the correct value, and about 30 to 1 that it is within $50 \%$ of the correct value. The smallest number of observations whose P.E. shall be regarded as trustworthy will be to a certain extent a matter of individual opinion, depending upon the odds which the individual is prepared to regard as practically equivalent to certainty. But one can at any rate regard 50 as a number sufficient to yield a fairly reliable P.E.

When the number of observations is small, say 10 , it is scarcely 
legitimate to regard the value of $0.6745 \sqrt{\frac{[v v]}{n-1}}$ as the P.E. of a single observation. It only yields an approximation, which cannot be regarded as a reliable one, to the value of the P.E.

There is another aspect of the question. The method of least squares is only strictly applicable to problems where the distribution of frequencies can be represented by a curve of the general form of the curve shown in fig. 3. The problem of determining the precision of the measurements is equivalent to determining the parameter $h$ which defines the exact form of the curve. But the value of $h$, or of the P.E. $r$, must obviously cease to have its original meaning when the curve of frequencies is dissymmetrical, or is ill-defined on account of the smallness of the number of observations. An examination of the frequency distributions shown in figs. 1, 4, 5, 6 and 7 , will show that it can scarcely be possible to gain any accurate idea of the true form of the frequency curve for a large number of observations from the chance distribution of a few observations.

It is only when the number of observations is large, say 50 or more, that the P.E. calculated from the formula

$$
r=0.6745 \sqrt{\frac{[v v]}{n-1}}
$$

can be regarded as a reliable measure. If we use this formula to calculate $r$ for a small number of observations, say 10, we cannot expect another observer, working under similar conditions, to obtain the same value of $r$ for similar observations.

When the number of observations is small, or when the curve of frequencies is dissymmetrical (as in fig. 7), the calculated P.E. can only be regarded as some kind of measure of the mutual agreement of the observations in the series, a small P.E. indicating that the disagreement between individual observations is small.

\section{A Comparison of the two Formulae for $\mathbf{r}$.}

It has been shown above that when $r$ is calculated from the squares of the residuals, the P.E. of $r$ is given by

$$
\frac{\text { P.E. of } r}{r}=\frac{\cdot 707}{\sqrt{n-1}} \text {. }
$$


Helmert* has shown, by an investigation which is too long and complicated to be included here, that when $r$ is calculated from Peters' formula

the M.S.E. of $r$ is given by

$$
r=0.8453 \frac{v]}{\sqrt{n(n-1)}},
$$

$$
\frac{\text { M.S.E. of } r}{r}=\sqrt{\frac{\pi-2}{2(n-1)}}=\frac{\cdot 755}{\sqrt{n-1}} .
$$

Thus the P.E. of $r$ calculated from the squares of the residuals is less than the P.E. of $r$ calculated from the sum of the residnals in the ratio $707: 755$ or $1: 1 \cdot 07$. The first methor of calculating $r$ is thus slightly better than the second, but the difference in their precision is not sufficiently great to justify the entire use of the first formula and the neglect of Peters' formula. Peters' formula has a very great advantage in that the labour involved in its use is by far less, particularly when the number of observations is great. And it is generally found that when the number of observations is fairly large, and the curve of errors is symmetrical, the two formulae for $r$ yield almost identical results. The two formulae have an equally strong theoretical basis, and when the results disagree, it is becanse the frequency distribution does not follow the normal law; and in this case the method of least squares should be applied with considerable caution.

The following practical consideration is very important. In a long series of observations it often happens that one or two observations are rejected as discordant. The retention of a discordant observation generally makes a considerable difference in the value of $\sqrt{[v v]}$, but a very much smaller difference in the value of $v$ ]. Thus when there are observations which we are doubtful about retaining, it is probably better to use Peters' formula.

* Astron. Nachrichten, Bd. 88, No. 2096-7. 


\section{CHAPTER IV}

\section{OBSERVATIONS OF DIFFERENT WEIGHT}

\section{The Weighting of Observations.}

We have hitherto regarded all our observations as having equal precision, or, as is more generally said, as having equal weight. It is now necessary to consider how our formulae must be modified when the observations are regarded as having unequal weight, and consequently as having unequal importance in the determination of the most plausible value of the unknown. The meaning of "weight" and its effect upon the least squares solution can be most clearly seen by the consideration of a simple example.

Let $x_{1}, x_{2}, x_{3}, x_{4}$ be four observed values of an unknown quantity $x$. There are four equations of condition:

$$
\begin{aligned}
& x-x_{1}=v_{1}, \\
& x-x_{2}=v_{2}, \\
& x-x_{3}=v_{3}, \\
& x-x_{4}=v_{4} .
\end{aligned}
$$

The most plausible value of the unknown is

$$
x=\frac{x_{1}+x_{2}+x_{3}+x_{4}}{4} .
$$

Now suppose the first three observations to be grouped together, yielding a value $x^{\prime}\left(=\frac{x_{1}+x_{2}+x_{3}}{3}\right)$ of the unknown for that group. Then there will be only two equations of condition:

$$
\begin{aligned}
& x-x^{\prime}=v^{\prime}, \\
& x-x_{4}=v_{4} .
\end{aligned}
$$


Since $x^{\prime}$ is the mean of three determinations, while $x_{4}$ is a single determination, we may say that $x^{\prime}$ has a weight 3 , while $x_{4}$ has unit or standard weight. The adopted value of the unknown may now be written

$$
x=\frac{x_{1}+x_{2}+x_{3}+x_{4}}{4}=\frac{3 x^{\prime}+x_{4}}{3+1} .
$$

If $x^{\prime}$ and $x_{4}$ were direct observations of such a nature that it could be decided from any consideration that three observations such as $x_{4}$ must be taken and combined in order to yield a result as valuable as $x^{\prime}$, we could still say that $x^{\prime}$ should have a weight 3 , while $x_{4}$ had the standard or unit weight. The adopted value of the unknown would still be written

$$
x=\frac{3 x^{\prime}+x_{4}}{3+1}
$$

The most plausible value of the unknown is derived by multiplying each observed value by its weight, adding together the products, and dividing the results by the sum of the weights.

The result may be generalised for any number of observations, with any assigned weights. If $x_{1}, x_{2}, \ldots, x_{n}$ be $n$ observations, whose weights are $p_{1}, p_{2}, \ldots, p_{n}$, respectively, the adopted value of the unknown $x$ is

$$
x=\frac{p_{1} x_{1}+p_{2} x_{2}+\ldots+p_{n} x_{n}}{p_{1}+p_{2}+\ldots+p_{n}}=\frac{[p x]}{[p]} .
$$

The adopted value of the unknown is called the "weighted mean." It may be noted in passing that this result is not altered when all the weights are increased or decreased in the same ratio.

In the simple case considered above, all that is meant by the weight 3 assigned to $x^{\prime}$ is that, on the average, three observations of unit weight must be combined in order to yield a result as good as $x^{\prime}$. Similarly, in the general case, the meaning of a weight $p_{r}$ assigned to an observation $x_{r}$, is that $p_{r}$ observations of unit weight must be combined in order to yield as reliable a result as $x_{r}$. Later on we shall have to consider the different methods of assigning weights to observations, but for the present we are only concerned with the modifications in the methods of solution produced by the difference in weights. 
The observational equations for a series of $n$ weighted observations may be written

$$
\left.\begin{array}{l}
x_{1}-x=v_{1} \quad \text { weight } p_{1} \\
x_{2}-x=v_{2} \quad, \quad p_{2} \\
\ldots \ldots \ldots \ldots \ldots \ldots \ldots \ldots \ldots \ldots \ldots \\
\cdots \ldots \ldots \ldots \ldots \ldots \ldots \ldots \ldots \ldots \\
x_{n}-x=v_{n} \quad, \quad p_{n}
\end{array}\right\}
$$

Let the M.S.E. of a hypothetical observational equation of unit weight be $\mu$. Then it follows from the definition of weight, that the mean values of the residuals are given by

$$
\begin{gathered}
v_{1}{ }^{2}=\frac{\mu^{2}}{p_{1}}, \quad v_{2}{ }^{2}=\frac{\mu^{2}}{p_{2}}, \text { etc., } \\
\mu^{2}=p_{1} v_{1}{ }^{2}=p_{2} v_{2}{ }^{2}=\ldots=p_{n} v_{n}{ }^{2} .
\end{gathered}
$$

or

From this it follows that the above equations of condition are reduced to equations of equal M.S.E., and therefore to equations of equal weight, when each equation is multiplied by the 'square root of the corresponding weight. The system of equations may then be written

$$
\left.\begin{array}{c}
\sqrt{p_{1}}\left(x_{1}-x\right)=\sqrt{p_{1}} \cdot v_{1} \\
\sqrt{p_{2}}\left(x_{2}-x\right)=\sqrt{p_{2}} \cdot v_{2} \\
\text { etc. }
\end{array}\right\}
$$

As all these equations have equal weight, the system may be solved by the ordinary method of least squares. The value of the unknown is obtained by making $\Sigma\left(p v^{2}\right)$ a minimum; i.e. by making

$$
p_{1}\left(x_{1}-x\right)^{2}+p_{2}\left(x_{2}-x\right)^{2}+\ldots \text { etc. }
$$

a minimum. Differentiating with respect to $x$, we obtain for the value of the weighted mean

$$
x=\frac{p_{1} x_{1}+p_{2} x_{2}+\ldots}{p_{1}+p_{2}+\ldots}=\frac{[p x]}{[p]},
$$

which agrees with the value previously derived.

The equations (B) above are all of unit weight, and therefore the true M.S.E. $\mu$ of any one of them is given by

$$
\mu=\sqrt{\frac{[p v v]}{n-1}} .
$$


It follows that if $r_{0}$ be the P.E. of a single equation of unit weight,

$$
r_{0}=0.6745 \sqrt{\frac{[p v v]}{n-1}} \text {, or } r_{0}=0.8453 \frac{\sqrt{p} \cdot v]}{\sqrt{n(n-1)}} \text {. }
$$

The P.E. of an observation of weight $p$ is $\frac{r_{0}}{\sqrt{p}}$; since such an observation. is equivalent to $p$ observations of unit weight and P.E. $r_{0}{ }^{*}$. The weighted mean is equivalent to the arithmetic mean of $[p]$ observations, and so its P.E. $r$ is given by

$$
r=\frac{r_{0}}{\sqrt{[p]}}=0.6745 \sqrt{\frac{[p v v]}{[p](n-1)}} .
$$

It was assumed in the original definition of weight that the $p$ 's were integral. It should be noted, however, that the values of both the weighted mean and its P.E. are independent of the actual values of the weights, and depend only on their relative values.

\section{Methods of Weighting.}

\section{(a) Arbitrary Scales.}

It sometimes happens that the external conditions vary irregularly during a series of observations, in such a way that, although the effeet upon the separate observations eannot be evaluated, yet the observer is able to decide that some of the observations are less affeeted than others. In such a ease it appears legitimate to attach greater importance to the less affected observations. This is done by assigning to these observations a relatively higher weight than the more affeeted observations, the relative values of the weights being determined by the observer according to some arbitrary scale which he sets up for himself. Thus an astronomical observer making a long series of observations extending over many nights may fairly attach different weights to the results of the separate nights according to the steadiness or unsteadiness of seeing. When once these weights have been assigned, the weighted mean and its P.E. can be immediately evaluated by the use of the formulae deduced above.

* This result may also be derived as follows. Since $r_{0}$ is the P.E. of $\sqrt{p_{r}} v_{r}$, the P.E. of $v_{r}$ is $\frac{r_{0}}{\sqrt{p_{r}}}$. 
The greatest disadvantage of this method of weighting lies in its arbitrary and personal nature, as, in general, two observers making precisely the same series of observations would not assign the same relative weights to the separate observations. Further, a computer reducing a set of observations may legitimately reject the weights assigned by the observer when he considers that the observations are more affected by other factors not considered by the observer, than by the factors on which the weights are based. I'hus, if an observer assigns weights to observations of the moon according to the steadiness of seeing, the computer may reject these weights if he finds that the observations are affected by the inequalities of the moon's limb to a greater extent than by the differences in seeing.

It is impossible to give any rules for the guidance of the inexperienced observer as to the formation of a scale of weights to represent the varying conditions during a series of observations. His safest plan is to observe only when external conditions are fairly stable, and to assign the same weights to all his observations, unless he has some very good reason for doing otherwise.

The general tendency of most observers is to overdo this type of weighting; i.e. to regard the bad observations as worse than they really are. "It appears that the longer time one is compelled to bestow, and does bestow, upon observations made under less favourable circumstances, in a great measure compensates external disadvantages; and that causes of errors of observation of which the observer himself has not been conscious, often influence him no less than those which obtrude themselves upon him*."

\section{(b) Number of Observations in Grouped Means.}

When it is required to combine a number of quantities each of which is the mean of a group of observations, nothing being known of the individual observations in each group, each groupmean is given a weight proportional to the number of observations in the group. The weighted mean thus obtained is clearly the same as the mean of all the observations. But the P.E. derived

* Ordnance Survey. Principal Triangulation. 
from grouped means may differ slightly from that derived from the actual observations.

(c) Weigliting by Probable Errors*.

Let $x_{1}, x_{n}, \ldots, x_{n}$ be separate determinations of a quantity $x$, where the P.E. of $x_{g}(s=1,2, \ldots, n)$ is known. The most probable value of $x$ is obtained by making

$$
\sum h_{8}{ }^{2}\left(x_{8}-x\right)^{2} \text { a maximum. }
$$

This is equivalent to making

$$
\Sigma \frac{\left(x_{8}-x\right)^{2}}{r_{8}^{2}} \text { a maximum. }
$$

Differentiating with respect to $x$, we obtain

where

$$
x=\frac{\left[\frac{1}{r_{8}{ }^{2}} \cdot x_{8}\right]}{\left[\frac{1}{r_{8}{ }^{2}}\right]}=\frac{\left[p_{8} x_{8}\right]}{\left[p_{8}\right]},
$$

This result may be interpreted as follows :

If it is necessary to combine a number of separate determinations of an unknown quantity, where the P.E. of each separate determination is known, the best result is obtained by assigning to each determination a weight inversely proportional to the square of its P.E.

This method of weighting is undoubtedly the best when it is possible to obtain trustworthy P.E.'s of the separate determinations, the number of observations on which each determination is based being not too small. When the number of observations is small there is always a danger of a run of luck causing the observations in a group to fall close together, so yielding a very small P.E., and consequently a very large weight. In such a case the computer. must decide whether the small P.E. represents a true P.E. or is small simply through the accidentally close agreement of the observations in a group.

* In all that follows the M. B.E. $\mu$ may be used instead of the P.E. $r$, as the basis of weighting.

B. 0 . 
The following description of a practical method of weighting, extracted from Wright and Hayford's Adjustment of Observations (page 76), is particularly instructive. "A long-continued series of observations will show the kind of work an instrument is capable of doing under favourable conditions; and if work is done only when the conditions are favourable, the P.E. derived from a certain number of results will generally fall within limits that can be assigned a priori. For example, with the Lake Survey primary theodolites, which read to single seconds, the tenths being estimated, the work of several seasons showed that the mean of from 16 to 20 results of the value of a horizontal angle, each result being the mean of a reading with telescope direct and a reading with telescope reverse, need not be expected to be greater than $0^{\prime \prime} 3$. If, therefore, after having measured a series of angles in a triangulation net with these instruments, the P.E.'s all fell within $\pm 0^{\prime \prime} \cdot 3$, it was considered sufficient to assign to each angle the same weight."

No general rules can be given as to the best methods of assigning weights. The computer must take into consideration all the information available concerning the external conditions at the time of observation, the possible presence of constant errors, the type of instrument used, the reputation of the observer for accurate work, as well as the computed P.E. With all these in mind he must use his own judgment as to the best method of procedure. The inexperienced computer must guard against assigning widely divergent weights to his observations, unless he has very strong grounds for so doing.

In assigning weights according to P.E. it is necessary to consider the possibility of a systematic or constant error being present in the set of observations, causing an error in the final result. For, since the P.E. only takes account of the accidental errors, it can only be regarded as a valid measure of the precision of a result when it is certain that the result is not affected by systematic errors. Thus in an attempt to combine the values of the solar parallax obtained by different methods, we must consider not only the calculated P.E., but also the possible sources of systematic errors. Newcomb states that the errors principally to be feared in a determination of the solar parallax are not the 
accidental errors treated by the method of least squares, but the systematic ones arising principally from personal equation and an imperfect reduction of the observations to the centre of the planet, or to the sun. It is therefore useless to assign relative weights based on the P.E.'s of the separate determinations. From a careful study of the systematic errors entering into the different estimates of the solar parallax, Newcomb assigned the weights shown in the table below. The weights bear no very close relation to the calculated P.E.'s.

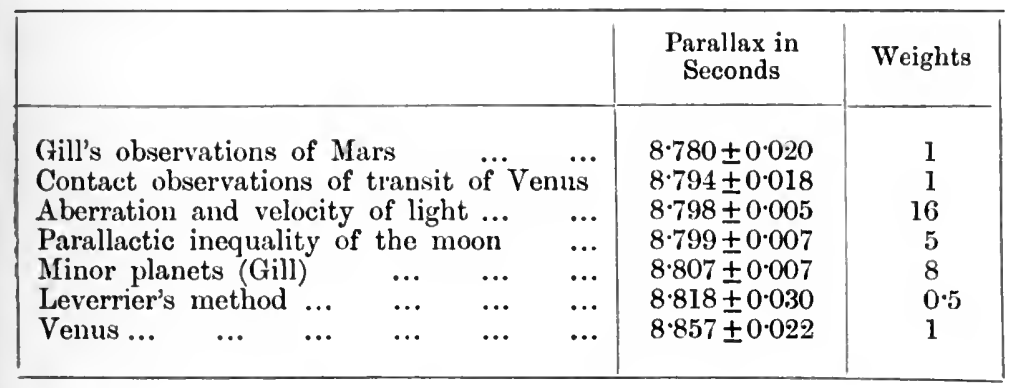

33. An alternative Method of Evaluating the Precision of the Weighted Mean when Weighting according to Probable Errors.

It has been shown above that if we wish to combine a number of determinations of any quantity when the P.E. of each separate determination is known, the weight to be given to each determination must be proportional to the inverse square of the probable error. If $x_{1}, x_{2}, \ldots, x_{n}$ be the $n$ determinations, whose P.E.'s are $r_{1}, r_{2}, \ldots, r_{n}$, the weight $p_{8}$ of the determination $x_{8}$ is given by

$$
p_{8}=\frac{1}{r_{8}^{2}} \text {. }
$$

The weighted mean can be evaluated by means of the equation

$$
x=\frac{\left[\frac{x_{8}}{r_{8}^{2}}\right]}{\left[\frac{1}{r r_{8}^{2}}\right]} .
$$


The P.E. of the weighted mean can be evaluated from the residuals by the use of the formula

$$
r=0.6745 \sqrt{\frac{[p v v]}{[p](n-1)}}
$$

But there is another way of approaching the question. The weighted mean $x$, defined by the equation

$$
x=\frac{\left[\frac{x_{s}}{r_{s}^{2}}\right]}{\left[\frac{1}{r_{s}^{2}}\right]},
$$

is a linear function of $n$ quantities $x_{1}, x_{2}$, etc., whose P.E.'s are known. Hence if $r$ be the P.E. of $x$,

or

$$
\begin{gathered}
r^{2}=\frac{\frac{1}{r_{1}{ }^{r_{1}}{ }^{2}+\frac{1}{r_{2}^{4}} r_{2}{ }^{2}+\ldots}}{\left(\frac{1}{r_{1}^{2}}+\frac{1}{r_{2}{ }^{2}}+\ldots\right)^{2}}, \\
\frac{1}{r^{2}}=\frac{1}{r_{1}^{2}}+\frac{1}{r_{2}{ }^{2}}+\ldots+\frac{1}{r_{n}{ }^{2}}
\end{gathered}
$$

The results derived from equations (2) and (3) will in general differ. For the first of these bases the calculation of $r$ upon the differences between the individual determinations $x_{1}, x_{2}$, etc.; while the second method neglects entirely these differences. There naturally arises the question, "which of the two methods of evaluating $r$ is the better?" The answer to this question depends to some extent upon the material under consideration. If the differences between the individual determinations which have to be combined are attributable to systematic errors entering into different determinations in different ways, it is clear that the P.E. of a determination can give no clear estimate of the reliability of that determination. In such a case equation (3) yields a value of $r$ which is of no use as a measure of the reliability of the result. We are then forced to use equation (2). If the number of determinations to be combined be small, say, 3 or 4 , and we have no reason to suspect the presence of systematic errors, it is better to 
use equation (3). In all other cases it is safer to use equation (2); i.e. to calculate the P.E. from the residuals.

\section{EXAMPLES.}

1. Given the following six determinations of the parallax of the star Lalande 21185, find the weighted mean and its P.E.

\begin{tabular}{|r|r|r|r|r|r|r|}
\hline Parallax & Weicht $(p)$ & \multicolumn{1}{|c|}{$x$} & $p x$ & \multicolumn{1}{c|}{$v$} & \multicolumn{1}{c|}{$v^{2}$} & \multicolumn{1}{c|}{$p v^{2}$} \\
\hline $0^{\prime \prime} \cdot 507$ & 8 & 107 & 856 & 104 & 10816 & 86528 \\
$\cdot 438$ & 5 & 38 & 190 & 35 & 1225 & 6125 \\
$\cdot 381$ & 2 & -19 & -38 & -22 & 484 & 968 \\
$\cdot 371$ & 8 & -29 & -232 & -32 & 1024 & 8192 \\
$\cdot 350$ & 13 & -50 & -650 & -53 & 2809 & 36517 \\
$\cdot 402$ & 20 & 2 & 40 & -1 & 1 & 20 \\
\hline & 56 & & 166 & & & 138350 \\
\hline
\end{tabular}

Let the parallax of the star be $0^{\prime \prime} \cdot 4+x^{\prime \prime} \times 10^{-3}$ Then the six values of $x$ are given in the third column. Each value of $x$ is multiplied by the corresponding weight, and the result written in the fourth column. The weighted mean gives for $x$,

$$
x=\frac{106}{56}=3 .
$$

The adopted parallax is therefore $0^{\prime \prime} \cdot 403$.

The residual $v$ obtained by subtracting the weighted mean from $x$ is written in the fifth column, $v^{2}$ in the sixth column, and $p v^{2}$ in the seventh column. The sun of the last column yields [ $p v v]$.

The P.E. of the weighted mean is

$$
0.6745 \sqrt{\frac{[p v v]}{[p](n-1)}}=0.6745 \sqrt{\frac{138350}{56 \times 5}}=15.0
$$

in units of the third decimal place.

The final result may be written

$$
0 " \cdot 403 \pm \cdot 015
$$


2. Find the weighted mean and its P.E, for the following determinations of the difference of longitude between two places :

\begin{tabular}{|r|r|r|r|r|r|r|}
\hline & Weights $(p)$ & $x$ & $p x$ & $v$ & $v^{2}$ & $p v^{2}$ \\
\hline $19^{\mathrm{m}} 1 \mathrm{~s} \cdot 42 \pm 0^{\mathrm{s}} \cdot 044$ & $1 \cdot 1$ & -3 & $-3 \cdot 3$ & -4 & 16 & $17 \cdot 6$ \\
$\cdot 37 \pm \cdot 037$ & $1 \cdot 6$ & -8 & $-12 \cdot 8$ & -9 & 81 & $129 \cdot 6$ \\
$\cdot 38 \pm \cdot 036$ & $1 \cdot 7$ & -7 & $-11 \cdot 9$ & -8 & 64 & $108 \cdot 8$ \\
$\cdot 45 \pm \cdot 036$ & $1 \cdot 7$ & 0 & 0 & -1 & 1 & $1 \cdot 7$ \\
$\cdot 60 \pm \cdot 046$ & $1 \cdot 0$ & 15 & $15 \cdot 0$ & 14 & 196 & $196 \cdot 0$ \\
$\cdot 55 \pm \cdot 045$ & $1 \cdot 0$ & 10 & $10 \cdot 0$ & 9 & 81 & $81 \cdot 0$ \\
$\cdot 57 \pm \cdot 047$ & $1 \cdot 0$ & 12 & $12 \cdot 0$ & 11 & 121 & $121 \cdot 0$ \\
\hline
\end{tabular}

The weights of the determinations are proportional to

$$
\frac{1}{(44)^{2}}, \frac{1}{(37)^{2}}, \frac{1}{(36)^{2}}, \frac{1}{(36)^{2}}, \frac{1}{(46)^{2}}, \frac{1}{(45)^{2}}, \frac{1}{(47)^{2}} \text {. }
$$

Let the weight of the last determination be made unity. 'Then the others become

or

$$
\begin{aligned}
& \left(\frac{47}{44}\right)^{2},\left(\frac{47}{37}\right)^{2},\left(\frac{47}{36}\right)^{2},\left(\frac{47}{36}\right)^{2},\left(\frac{47}{46}\right)^{2},\left(\frac{47}{45}\right)^{2}, 1 . \\
& 1 \cdot 1, \quad 1 \cdot 6, \quad 1 \cdot 7, \quad 1 \cdot 7, \quad 1 \cdot 0, \quad 1 \cdot 0, \quad 1 \cdot 0 .
\end{aligned}
$$

These weights are written down in the second column of the table. The longitude difference is assumed to be $19^{\mathrm{m}} 1^{\mathrm{s} \cdot 45}+x \times 0^{\prime \prime} \cdot 01$. The separate determinations of $x$ are written in the third column, and the values of $p x$ in the fourth column. The sum of the fourth column, divided by the sum of the weights, gives $\frac{9 \cdot 0}{9 \cdot 1}$ or $1 \cdot 0$ for the weighted mean of $x$. The residual $v$ is $x-1$. The rest of the table is self-explanatory.

The P.E. of the weighted mean

$$
=0.6745 \sqrt{\frac{655 \cdot 7}{9 \cdot 1 \times 6}}=2 \cdot 3
$$

in units of the second decimal place.

The final result may therefore be written

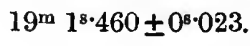


3. From the following determinations of the parallax of 61 Cygni find the weighted mean, and its P.E. :

$$
\begin{array}{rr}
0^{\prime \prime} \cdot 316 \pm 0^{\prime \prime} \cdot 016 \\
\cdot 216 \pm & .029 \\
\cdot 333 \pm & .035 \\
\cdot 290 \pm & .009 \\
\cdot 300 \pm & .007 \\
\cdot 387 \pm & .015 \\
\cdot 328 \pm & .029 \\
\cdot 298 \pm & .005 \\
\cdot 238 \pm & .020 \\
\cdot 388 \pm & .017
\end{array}
$$

[Give each determination a weight proportional to $\frac{1}{(\text { P.E. })^{2}}$, and evaluate P.E. of the weighted mean from the residuals.]

4. An angle was determined in three separate years, the following results being obtained :

$$
\begin{array}{r}
149^{\circ} 16^{\prime} 51^{\prime \prime} \cdot 48 \pm 0^{\prime \prime} \cdot 45 \\
48 \cdot 47 \pm 0 \cdot 28 \\
49 \cdot 72 \pm 0 \cdot 25
\end{array}
$$

Find the most probable measure of the angle.

[In this case the differences between the individual observations are greatly in excess of the P.E.'s. There are obviously systematic errors present, so that the P.E.'s do not represent the whole error. It is probably

\begin{tabular}{|c|c|c|c|c|}
\hline$l^{\prime \prime} \cdot 27 \pm 0^{\prime \prime} \cdot 11$ & determ & from & 4 & 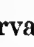 \\
\hline $1 \cdot 00 \pm 0 \cdot 10$ & , & " & 3 & ", \\
\hline $0 \cdot 91 \pm 0 \cdot 07$ & . " & $"$ & 4 & $"$ \\
\hline $1 \cdot 12 \pm 0 \cdot 13$ & " & ", & 6 & $"$ \\
\hline $1 \cdot 30 \pm 0 \cdot 11$ & $"$ & " & 6 & $"$ \\
\hline $1 \cdot 42 \pm 0 \cdot 19$ & $"$ & $"$ & 6 & $"$ \\
\hline $1 \cdot 45 \pm 0 \cdot 15$ & 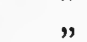 & $"$ & 6 & ", \\
\hline
\end{tabular}
preferable in this case to adopt the simple A.M. as the value of the angle.]

5. If the probable accidental error of observing a star is $0^{\prime \prime} \cdot 30$, and the probable quasi-systematic error of a gradation is $0^{\prime \prime} \cdot 20$, how would you combine 7 observations of one star with 3 observations of another star?

Referring to page 51, we find that

$$
\begin{aligned}
& \text { (P.E. })^{2} \text { of mean of } 7 \text { observations }=(\cdot 20)^{2}+\frac{1}{7}(\cdot 30)^{2} \text {, } \\
& \text { (P.E. })^{2} \text { of mean of } 3 \text { observations }=(\cdot 20)^{2}+\frac{1}{3}(\cdot 30)^{2} .
\end{aligned}
$$

6. From the table of determinations of the solar parallax given on page 67 , using the weights assigned by Newcomb, show that the weighted mean is

$$
8^{\prime \prime} \cdot 802 \pm 0^{\prime \prime} \cdot 005 \text {. }
$$

7. The difference between the observed values and the assumed value of an angle is given by the following set of determinations : 
How would you combine these values to obtain the most probable value (a) when the above probable errors are given simply by the discordances between the individual observations of each group, and $(b)$ when in each case the probable error is determined from a long series of which each member of the above groups is a sample?

In the former case, determine the most probable value of the angle and its probable error.

[In the first case the observations are to be weighted according to the number of observations in each group, while in the second case the P.E.'s must also be tiken into account.]

\section{MISCELLANEOUS EXAMPLES INVOLVING ONE UNKNOWN.}

1. If the error of a clock determined at time

$$
\begin{array}{r}
0^{\mathrm{h}} \text { is } a_{1}^{8} \pm r_{1}, \\
12^{\mathrm{h}} \text { is } a_{2}^{8} \pm r_{2},
\end{array}
$$

find the clock error aud its P.E. for an interpolated time $8^{\mathrm{h}}$.

2. The equatorial velocity of the sun, determined by a spectroscopic method, yielded the following results:

\begin{tabular}{|c|c|c|}
\hline Element & Number of lines & $\mathrm{Km} . / \mathrm{sec}$. \\
\hline & & \\
\hline $\mathrm{Fe}$ & 18 & $1 \cdot 857$ \\
$\mathrm{Ti}$ & 8 & $1 \cdot 883$ \\
$\mathrm{Cr}$ & 6 & $1 \cdot 883$ \\
$\mathrm{Sc}$ & 5 & $1 \cdot 817$ \\
$\mathrm{Ca}$ & 3 & $1 \cdot 845$ \\
$\mathrm{~V}$ & 3 & $1 \cdot 870$ \\
$\mathrm{Zr}$ & 3 & 1.915 \\
$\mathrm{Mn}$ & 2 & 1.819 \\
$\mathrm{Mg}$ & 1 & $1 \cdot 989$ \\
$\mathrm{Ni}$ & 1 & $1 \cdot 848$ \\
\hline
\end{tabular}

Combine these values $(a)$ giving all elements equal weight, $(b)$ giving each element a weight equal to the number of lines measured.

3. $2 n+1$ observations are made, each with the same P.E. Show that the probability of the error of the median being between $x$ and $x+d x$ is

where

$$
\begin{aligned}
& \frac{2 n+1 !}{n ! n !}\left(\frac{1}{4}-a^{2}\right)^{n} d a, \\
& a=\frac{h}{\sqrt{\pi}} \int_{0}^{x} e^{-h^{2} t^{2}} d t .
\end{aligned}
$$

In the case of five observations, show that the chance of the error of the median being numerically greater than $r$ is $\frac{53}{256}$. 
4. The probability of an event lappening once in one trial is $p$, so that in $m$ trials the event happens on an average $m p$ times. Find the P.E. of the number $m p$.

Let $q$ be the probability of the event not happening, so that

The whole series is given by

$$
q=1-p .
$$

$$
(p+q)^{m}=p^{m}+m p^{m-1} q+\ldots+q^{m} .
$$

This may be interpreted as follows: The frequency of the event happening $m$ times is $p^{m}$, of its happening $(m-1)$ times is $m p^{m-1} q$, etc. The separate terms of the Binomial Series give the frequeney distribution of the different possible numbers of successes in $m$ trials. Or if $x$ be the number of times the event occurs in $m$ trials, we have the following frequency distribution:

$$
\begin{array}{ll}
x=m, & f=p^{m}, \\
x=m-1, & f=m p^{m-1} q, \\
x=m-2, & f=\frac{m \cdot m-1}{1 \cdot 2} p^{m_{-}-2} q^{2}, \\
\quad \text { etc., } & \\
x=0, \quad & f=q^{m} .
\end{array}
$$

Taking the origin at $x=m$, we find,

$$
\begin{aligned}
& \text { mean value }=m p^{m-1} q+\frac{m \cdot m-1}{1 \cdot 2} p^{m-2} q^{2}+\ldots \\
& =m q(p+q)^{m-1} \\
& =m q . \\
& \Sigma f(x-m)^{2}=m p^{m-1} q+2 m(m-1) p^{m-2} q^{2}+\ldots+m^{2} q^{m} \\
& =m p^{m-1} q+m(m-1) p^{m-2} q^{2}+\ldots+m q^{m} \\
& =m q+m(m-1) q^{2} . \\
& = \\
& =\Sigma f(x-m p)^{2}=\Sigma f(x-m)^{2}-(\text { mean value })^{2} \\
& =m q+m(m-1) q^{2}-m^{2} q^{2}=m p q,
\end{aligned}
$$

But (M.S.E. $)^{2}$

P.E. of $m p$

$$
=\cdot 6745 \sqrt{m p q} \text {. }
$$

The use of the formula will be perhaps best shown by a simple application. It has been stated that in the British Isles the proportion of male to female children is 1050 to 1000 . Hence the probability that a child should be male is $\frac{1}{2} 8 \frac{5}{50}$. Of 100,000 births the number of male children should be

The probable error of this estimate is

$$
\frac{105}{205} \times 100,000 \text { or } 51,219 \text {. }
$$

$$
\sqrt{100,000 \times \frac{385}{2} 8 \times \frac{100}{200}} \text { or } 158 \text { approximately. }
$$

If it were found that among 100,000 children born in this country, 51,500 were male, the deviation from the expected value would be only about twice the P.E., and need not be regarded as abnormal. But if among 10,000,000 children $5,150,000$ were male, the deviation from the expected value would be 
28,100 , while the P.E. of the deviation would be 1,580 . The actual deriation would be about 18 times the P.E., and we should conclude that the normal ratio of male to female children had been definitely changed.

5. Given that the P.E. of a single observation is 14 , how many observations must be taken and combined in order that the P.E. of the mean shall be less than 02 ?

6. A series of 100 observations of an angle gives for the P.E. of a single observation $1^{\prime} \cdot 75$. What is the probability that the error of the mean is not greater than $\cdot 25$ ?

7. An angle is measured 100 times, and the p.E. of a single observation is $1^{\prime} \cdot 75$. How many errors will be greater than $0^{\prime} \cdot 25$ and less than $1^{\prime} \cdot 25$ ?

8. If the P.E. of a single observation is 1.5 , how many observations must - be combined in order that the odds may be 3 to 1 that the mean is within .25 of truth? 


\section{CHAPTER V}

THE GENERAL PROBLEM OF THE ADJUSTMENT OF INDIRECT OBSERVATIONS INVOLVING MORE THAN ONE UNKNOWN QUANTITY

34. In the cases hitherto considered, the problem has been to find the most probable value of an unknown quantity, given a number of direct observations of that quantity. The arithmetic mean was adopted as the best value of the unknown. We must now consider the case where the quantity measured is not itself the unknown whose value is required, but is expressible as a function (not of necessity linear) of a number of unknown quantities. The problem may be briefly stated thus: Given a number of measurements of certain functions of a number of unknowns, to find the values of the unknowns, and their probable errors.

Let the $n$ observed quantities be $M_{1}, M_{2}, \ldots, M_{n}$; and let their unknown errors be $v_{1}, v_{2}, \ldots, v_{n}$. Then it is given that $M_{1}+v_{1}$, $M_{2}+v_{2}$, etc. can be accurately expressed as functions of the unknowns $X, Y, Z$, etc.,

$$
\begin{aligned}
& \begin{array}{l}
f_{1}(X, Y, Z, \ldots)=M_{1}+v_{1} \\
f_{2}(X, Y, Z, \ldots)=M_{2}+v_{2}
\end{array} \\
& f_{2}(X, Y, Z, \ldots)=M_{2}+v_{2} \\
& \text { (n................................... }
\end{aligned}
$$

There will be one equation of this form for each observation; and in the problems with which we shall have to deal, the number $n$ of equations in (1) will be greater than the number $m$ of unknowns. 
Now suppose approximate values of the unknowns to be known, or to have been deduced by solving a sufficient number of the equations in (1). Let these approximate values be $X_{0}, Y_{0}, Z_{0}$, etc., and let

$$
X=X_{0}+x, \quad Y=Y_{0}+y, \quad Z=Z_{0}+z, \text { etc., }
$$

where it may be assumed that the corrections $x, y, z$, etc. are small, so that their squares may be neglected.

The first equation in (1) may then be written

$$
\begin{aligned}
& f_{1}\left(X_{0}, Y_{0}, Z_{0}, \ldots\right)+x \frac{\partial f_{1}}{\partial X_{0}}+y \frac{\partial f_{1}}{\partial \bar{Y}_{0}}+z \frac{\partial f_{1}}{\partial Z_{0}}+\ldots=M_{1}+v_{1} \\
& \frac{\partial f_{1}}{\partial X_{0}}=a_{1}, \quad \frac{\partial f_{1}}{\partial Y_{0}}=b_{1}, \text { etc., } \\
& \frac{\partial f_{2}}{\partial X_{0}}=a_{2}, \quad \frac{\partial f_{2}}{\partial Y_{0}}=b_{2}, \text { etc., } \\
& \text {................................., } \\
& -M_{1}+f_{1}\left(X_{0}, Y_{0}, Z_{0}, \ldots\right)=l_{1}, \\
& -M_{2}+f_{2}\left(X_{0}, Y_{0}, Z_{0}, \ldots\right)=l_{2} \text {, }
\end{aligned}
$$

Then equations (1) may be written

$$
\left.\begin{array}{l}
a_{1} x+b_{1} y+c_{1} z+\ldots-l_{1}=v_{1} \\
a_{2} x+b_{2} y+c_{2} z+\ldots-l_{2}=v_{2} \\
\ldots \ldots \ldots \ldots \ldots \ldots \ldots \ldots \ldots \ldots \ldots \ldots \ldots \ldots \ldots \ldots \ldots \ldots \ldots \ldots \ldots \ldots \ldots \ldots \ldots \ldots \ldots \ldots \ldots \ldots \ldots \ldots \ldots \ldots \ldots
\end{array}\right\}
$$

where the $a$ 's, $b$ 's, $c$ 's, $l$ 's, etc. are known.

Equations (1) or (2) are called the "observational equations." The problem has now been reduced to the case where the equations are all linear. If the values of $x, y, z$, etc. obtained by solving equations (2) be small, we may rest content with our solution; but if some of them should be large, it may be necessary to repeat the solution, taking the results of the first solution as approximate values of $X, Y, Z$, etc. It is found in practice that the approximation step saves considerable labour even in cases where the original observational equations are strictly linear. 


\section{Formation of the Normal Equations.}

If the observational equations all have the same weight, or are liable to the same mean error, the same discussion as for one unknown quantity will apply (see $\$ 13$ and 14). The probability of the occurrence of a residual $v$ may be written

$$
C e^{-h^{2} v^{2}} \text {. }
$$

The probability of the coexistence of the system of residuals $v_{1}, v_{2}, \ldots, v_{n}$ may be written

$$
C^{n} e^{-h^{2}[v v]} \text {. }
$$

The most probable values of the unknown will be such as to make this probability a maximum. The expression is greatest when $[v v]$ is least, and so the most probable values of the unknowns are given by the condition that

$$
[v v]=\text { a minimum, }
$$

or

$$
\sum_{r=1}^{r=n}\left(a_{r} x+b_{r} y+\ldots-l_{r}\right)^{2}=\text { a minimum. }
$$

The conditions for a minimum are obtained by equating to zero the differential coefficients of this expression with respect to $x, y$, etc.,

$$
\left.\begin{array}{r}
a_{1}\left(a_{1} x+b_{1} y+\ldots-l_{1}\right)+a_{2}\left(a_{2} x+b_{2} y+\ldots-l_{2}\right)+\ldots=0 \\
b_{1}\left(a_{1} x+b_{1} y+\ldots-l_{1}\right)+b_{2}\left(a_{2} x+b_{2} y+\ldots-l_{2}\right)+\ldots=0
\end{array}\right\}
$$

Collecting coefficients, we may write these equations in the form

$$
\left.\begin{array}{l}
{[a a] x+[a b] y+[a c] z+\ldots-[a l]=0 \equiv \xi} \\
{[a b] x+[b b] y+[b c] z+\ldots-[b l]=0 \equiv \eta} \\
{[a c] x+[b c] y+[c c] z+\ldots-[c l]=0 \equiv \zeta}
\end{array}\right\}
$$

These equations are called the normal equations. There is one normal equation corresponding to each unknown, and our problem has therefore been reduced to that of solving a set of linear equations whose number is the same as the number of unknowns. The solution of the normal equations gives the most 
probable values of the corrections $x, y, z$, etc., and from these corrections the values of the original unknowns $X, Y, Z$, etc. can be immediately deduced.

Since the coefficients of the normal equations are symmetrical about the principal diagonal, it is convenient to write the normal equations in the following abbreviated form, with half the cross products omitted,

$$
\left.\begin{array}{r}
{[a a] x+[a b] y+[a c] z+\ldots-[a l]=0} \\
+[b b] y+[b c] z+\ldots-[b l]=0 \\
+[c c] z+\ldots-[c l]=0
\end{array}\right\}
$$

There are $m$ normal equations when the number of unknowns $X, Y, Z$, etc. is $m$. The total number of products to be estimated is $\frac{1}{2} m(m+3)$, made up of $m$ products [aa], [bb], etc., $m$ products $[a l],[b l]$, etc., and $\frac{1}{2} m(m-1)$ products $[a b],[b c],[c a]$, etc.

Equations (3) may be written

$$
[a v]=0=[b v]=[c v]=\ldots .
$$

corresponding to the relation $[v]=0$ obtained in the case of one unknown quantity. These relations afford a check upon the values of $v_{1}, v_{2}$, etc. obtained from the solution of the equations.

If the observational equations are liable to different mean errors, or in other words have different weights $p_{1}, p_{2}$, etc., the equations (2) above must be multiplied by $\sqrt{p_{1}}, \sqrt{p_{2}}$, etc., respectively, so as to reduce them to equations of equal weight. Save for this multiplication it is not permissible to multiply any observational equation by an arbitrary factor. It is then necessary to make

$$
\text { [pvv] a minimum. }
$$

This condition leads to the set of normal equations

$$
\left.\begin{array}{l}
{[p a a] x+[p a b] y+[p a c] z+\ldots-[p a l]=0} \\
{[p a b] x+[p b b] y+[p b c] z+\ldots-[p b l]=0}
\end{array}\right\}
$$

with the conditions

$$
[p a v]=[p b v]=\ldots=0
$$


If we slightly revise our notation so that

$$
\begin{aligned}
& {[a a] \text { stands for } \Sigma p a^{2} \text {, }} \\
& {[a b] \quad " \quad \text { etc., }}
\end{aligned}
$$

then equations ( $\left.4^{\prime}\right)$ and $\left(6^{\prime}\right)$ are included in equations (4) and (6), and the same method of solution can be applied in each case. In all that follows we shall take account of possible differences in weight by regarding $[a a],[a b]$, etc. as $\Sigma p a^{2}, \Sigma p a b$, etc.

\section{Independence of the Normal Equations.}

If the normal equations are not all independent, it will be. possible to derive any one of them by combining some or all of the others, and the solution of equations (4) will not lead to a determinate solution for $x, y, z$, etc. In this case it must be possible to find $m$ constants, the ratios of $f, g, h, \ldots, k$, so that

$$
f \xi+g \eta+h \zeta+\ldots+k=0,
$$

where $\xi, \eta, \zeta$, etc.. are written for the normal equations (4), and $m$ is the number of unknowns $x, y, z$, etc.

Collecting the coefficients of $x, y, z$, in the last equation, we find

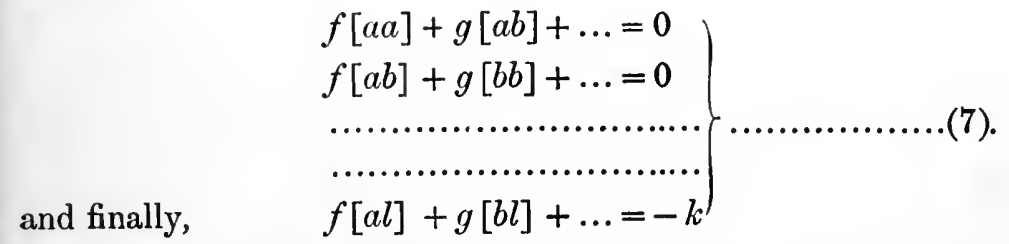

Multiplying the first $m$ of these equations by $f, g$, $h$, etc. respectively, and adding the products, we find

$$
\sum_{r=1}^{r=n}\left(f a_{r}+g b_{r}+\ldots\right)^{2}=0,
$$

or

$$
\left.\begin{array}{c}
f a_{1}+g b_{1}+\ldots=0 \\
f a_{2}+g b_{2}+\ldots=0 \\
\ldots \ldots \ldots \ldots \ldots \ldots \ldots \\
\ldots \ldots \ldots \ldots \ldots \ldots \ldots . . .
\end{array}\right\}
$$

There will be one of these equations for each observational 
equation. Multiplying equations ( 8 ) by $l_{1}, l_{2}$, etc. and adding, we find

$$
f[a l]+g[b l]+\ldots=0=-k \text { from }(7) .
$$

It follows from equations (8) that there cannot be more than $(m-1)$ independent observational equations; for equations (8) will yield a relation between the coefficients of any $m$ of these equations. The problem of solving for $x, y, z$, etc. is then indeterminate from the beginning.

The above discussion shows that if the normal equations are not independent, there could not have been $m$ independent observational equations. We infer that if there are at least $m$ independent observational equations, there will result $m$ independent normal equations, the solution of which yield determinate values of the $m$ unknowns. This may be otherwise stated as follows,If there are sufficient observational equations to determine or over-determine the solution, the normal equations yield a determinate solution.

The condition of independence is in general satisfied in the problems which arise in practice. We can then proceed to the formation and solution of the normal equations.

\section{Checks on the Formation of the Normal Equations.}

Before the normal equations can be written in a complete form, it is necessary to compute the products $[a a],[b b],[a b],[a l]$, etc. It is necessary to check the formation of these products. The most convenient form of check is the following.

Let

$$
a_{r}+b_{r}+\ldots+l_{r}=s_{r} .
$$

Then multiplying each $s$-equation by the corresponding $a$, and adding, we find

And similarly

$$
[a a]+[a b]+\ldots+[a l]=[a s] .
$$

$$
\begin{gathered}
{[a b]+[b b]+\ldots+[b l]=[b s]} \\
\text { etc. }
\end{gathered}
$$

Each of these equations is a check on the sum of the coefficients of one of the normal equations. The calculation of $[a s],[b s]$, etc. yields a double check on each of the cross-products. The 
additional work involved is very slight. We evaluate $s_{r}$ for each of the observational equations, and in the subsequent work treat $s_{r}$ in the same way as any other coefficient.

The work of computing the products is carried out with the aid of tables of squares, of products, or of logarithms, or with an arithmometer, a machine for performing multiplication and division. If the work is to be carried on by the aid of tables of squares, the products $[a b]$ etc. can be derived as follows:

$$
\begin{aligned}
(a+b)^{2} & =a^{2}+b^{2}+2 a b ; \\
\therefore 2[a b] & =\left[(a+b)^{2}\right]-[a a]-[b b] .
\end{aligned}
$$

Since $[a a]$ and $[b b]$ have to be evaluated in any case, the evaluation of $[a b]$ is simply replaced by the evaluation of $\left[(a+b)^{2}\right]$.

In any extensive piece of work involving a large number of observational equations, or a large number of unknowns, it is a great saving of labour to carry on the work in a fixed form, so that the whole of the work, including the application of the checks, shall be as uniform and mechanical as possible. ' It will also be necessary to have a system of checks at different stages in the process of solving the normal equations, so that any arithmetical error shall be immediately detected.

A great deal of labour can be saved in some cases by selecting new units of the unknowns, in such a way that the coefficients in the equations shall all be of the same order of rnagnitude.

38. It is sometimes possible to effect a considerable simplification of the work of solution by means of a simple substitution; e.g. when the observational equations are of the form

$$
a x+b x y=l,
$$

the solution is facilitated by the substitution

$$
u=x y \text {. }
$$

The observational equations then become

$$
a x+b u=l,
$$

and the solution can be carried out in the usual way. The P.E. of an observational equation of unit weight can be calculated from the residuals, and $r_{u}$ and $r_{x}$ deduced by the ordinary method. (See next chapter.)

B. 0 . 
Then, since

$$
\begin{gathered}
y=\frac{u}{x}, \\
d y=\frac{d u}{x}-\frac{u}{x^{2}} d x, \\
r_{y}^{2}=\frac{r_{u}^{2}}{x^{2}}+\frac{u^{2}}{x^{4}} r_{x}^{2}=\frac{r_{u}^{2}}{x^{2}}+\frac{y^{2}}{x^{2}} r_{x}^{2}, \\
r_{y}=\frac{1}{c} \sqrt{r_{u}^{2}+y^{2} r_{x}^{2} .}
\end{gathered}
$$

This method of introducing a new unknown dispenses with the approximation which would otherwise be necessary in the first step of the solution, by reducing the equations of condition to a linear form.

\section{The Formation of the Normal Equations.}

Example 1. Given the four equations*,

$$
\begin{aligned}
x-y+2 z-3 & =0, \\
3 x+2 y-5 z-5 & =0, \\
4 x+y+4 z-21 & =0 \\
-x+3 y+3 z-14 & =0
\end{aligned}
$$

it is required to find the most probable values of $x, y, z$.

If we solve the first three of these equations, we find

$$
x=2 \frac{4}{7}, \quad y=3 \frac{2}{7}, \quad z=1 \frac{6}{7} \text {. }
$$

\begin{tabular}{|c|c|c|c|c|}
\hline $\begin{array}{r}a \\
1 \\
3 \\
4 \\
-1\end{array}$ & $\begin{array}{r}b \\
-1 \\
2 \\
1 \\
3\end{array}$ & $\begin{array}{r}c \\
2 \\
-5 \\
4 \\
3\end{array}$ & $\begin{array}{r}l \\
3 \\
5 \\
21 \\
14\end{array}$ & $\begin{array}{r}s \\
5 \\
5 \\
30 \\
19\end{array}$ \\
\hline$a a$ & $a b$ & $a c$ & al & as \\
\hline $\begin{array}{r}1 \\
9 \\
16 \\
1\end{array}$ & $\begin{array}{r}-1 \\
6 \\
4 \\
-3\end{array}$ & $\begin{array}{r}2 \\
-15 \\
16 \\
-3\end{array}$ & $\begin{array}{r}3 \\
15 \\
84 \\
-14\end{array}$ & $\begin{array}{r}5 \\
15 \\
120 \\
-19\end{array}$ \\
\hline 27 & 6 & 0 & 88 & 121 \\
\hline
\end{tabular}

Substituting these values in the last equation, we find

$$
-x+3 y+3 z-14=-\frac{8}{7},
$$

and so the fourth equation is not satisfied. We therefore proceed to form the normal equations.

The formation of the normal equations is best carried out in tabular form. The coefficients of the observational equations are first written down, and the products formed in turn.

* Gauss, Theoria Motus, § 184. 


\begin{tabular}{|c|c|c|c|c|}
\hline$a b$ & $\begin{array}{c}b b \\
1 \\
4 \\
1 \\
9\end{array}$ & $\begin{array}{r}b c \\
-2 \\
-10 \\
4 \\
9\end{array}$ & $\begin{array}{r}b l \\
-3 \\
10 \\
21 \\
42\end{array}$ & $\begin{array}{r}b s \\
-5 \\
10 \\
30 \\
57\end{array}$ \\
\hline 6 & 15 & 1 & 70 & 92 \\
\hline$a c$ & $b c$ & $\begin{array}{r}c c \\
4 \\
45 \\
16 \\
9\end{array}$ & $\begin{array}{r}c l \\
6 \\
-25 \\
84 \\
42\end{array}$ & $\begin{array}{r}c 8 \\
10 \\
-25 \\
120 \\
57\end{array}$ \\
\hline 0 & 1 & 54 & 107 & 162 \\
\hline al & $u l$ & $c l$ & $\begin{array}{r}\| \\
9 \\
9 \\
25 \\
441 \\
196\end{array}$ & $\begin{array}{r}l s \\
15 \\
25 \\
630 \\
266\end{array}$ \\
\hline 88 & 70 & 107 & 671 & 936 \\
\hline
\end{tabular}

Checks are applied to all the sums as they are formed.

The normal equations may then be written

$$
\begin{aligned}
27 x+6 y & =88, \\
6 x+15 y+z & =70, \\
y+54 z & =107 .
\end{aligned}
$$

No special method is necessary for the solution of these equations. Substituting for $x$ and $z$ in the second equation, we find

$$
\frac{6}{27}(88-6 y)+15 y+\frac{1}{54}(107-y)=70,
$$

giving

$$
y=3 \cdot 55 \text {. }
$$

Substituting this value for $y$ in the first and third equations, we find

$$
x=2 \cdot 47, \quad y=3 \cdot 55, \quad z=1 \cdot 92 .
$$

The discussion of the P.E.'s of $x, y, z$ will be found on page 107 .

The quantity $[l l]$ evaluated above is not essential to the formation of the normal equations, but may be required later, in the discussion of P.E.'s. 
Example 2. Given a set of 14 observational equations of the form

$$
a \cdot x+b y=l
$$

with weight $p$, the values of $a, b, l$, and $p$ being given in the table below, form the normal equations.

\begin{tabular}{|r|r|r|r|r|}
\hline \multicolumn{1}{|c|}{$a$} & \multicolumn{1}{c|}{$b$} & \multicolumn{1}{c|}{$l$} & \multicolumn{1}{c|}{$s$} & $p$ \\
\hline & & & & \\
\hline 0.47 & 1.38 & -0.68 & 1.17 & 5 \\
1.43 & 0.02 & 0.75 & 0.80 & 4 \\
0.15 & -1.06 & 0.60 & -0.31 & 3 \\
-0.98 & -0.69 & 0.10 & -1.57 & 3 \\
-0.82 & -1.20 & 1.05 & -0.97 & 4 \\
-0.88 & -1.25 & -1.00 & -3.13 & 3 \\
-1.58 & -0.20 & -0.32 & -2.10 & 5 \\
-1.12 & 0.62 & -0.27 & -0.77 & 3 \\
-1.27 & -0.17 & 1.47 & 0.03 & 3 \\
-1.10 & 0.36 & -0.06 & -0.80 & 3 \\
-1.14 & 1.19 & -0.36 & -0.31 & 5 \\
-0.96 & 0.62 & -0.36 & -0.70 & 3 \\
-0.13 & 1.58 & 0.02 & 1.47 & 5 \\
0.62 & 1.00 & 0.50 & 2.12 & 3 \\
\hline
\end{tabular}

We have to evaluate $[p a a],[p a b],[p a l]$, etc.

The first step is to rewrite the above table, with each line multiplied by the corresponding weight.

\begin{tabular}{|r|r|r|r|}
\hline \multicolumn{1}{|c|}{$p c$} & \multicolumn{1}{|c|}{$p l$} & \multicolumn{1}{|c|}{$p l$} & \multicolumn{1}{c|}{$p s$} \\
\hline & & & \multicolumn{1}{c|}{} \\
\hline 2.35 & 6.90 & -3.40 & 5.85 \\
5.72 & 0.08 & 3.00 & 3.20 \\
0.45 & -3.18 & 1.80 & -0.93 \\
-2.94 & -2.07 & 0.30 & -4.71 \\
-3.28 & -4.80 & 4.20 & -3.88 \\
-2.64 & -3.75 & -3.00 & -9.39 \\
-7.90 & -1.00 & -1.60 & -10.50 \\
-3.36 & 1.86 & -0.81 & -2.31 \\
-3.81 & -0.51 & 4.41 & 0.09 \\
-3.30 & 1.08 & -0.18 & -2.40 \\
-5.70 & 5.95 & -1.80 & 1.55 \\
-2.88 & 1.86 & -1.08 & -2.10 \\
-0.65 & 7.90 & 0.10 & 7.35 \\
1.86 & 3.00 & 1.50 & 6.36 \\
\hline
\end{tabular}




\begin{tabular}{|c|c|c|c|}
\hline paa & pab & $p a l$ & pas \\
\hline $1 \cdot 105$ & $3 \cdot 2+3$ & $-1 \cdot 598$ & $2 \cdot 750$ \\
\hline $8 \cdot 180$ & $\cdot 114$ & $4 \cdot 290$ & $12 \cdot 584$ \\
\hline .068 & -477 & $\cdot 270$ & $-\cdot 139$ \\
\hline $2 \cdot 881$ & $2 \cdot 029$ & $-\cdot 294$ & $4 \cdot 616$ \\
\hline $2 \cdot 690$ & $3 \bullet 936$ & $-3 \cdot+44$ & $3 \cdot 182$ \\
\hline $2 \cdot 3 \cdot 23$ & $3 \cdot 300$ & $2 \cdot 640$ & $8 \cdot 263$ \\
\hline $12 \cdot 482$ & $1 \cdot 580$ & $2 \cdot 528$ & 16.590 \\
\hline $3 \cdot 763$ & $-2 \cdot 083$ & :907 & 2587 \\
\hline $4 \cdot 839$ & $\cdot 648$ & $-5 \cdot 601$ & $-\cdot 115$ \\
\hline $3 \cdot 630$ & $-1 \cdot 188$ & $\cdot 198$ & $2 \cdot 640$ \\
\hline $6 \cdot 498$ & $-6 \cdot 783$ & $2 \cdot 052$ & $1 \cdot 767$ \\
\hline $2 \cdot 765$ & $-1 \cdot 786$ & $1 \cdot 037$ & $2 \cdot 016$ \\
\hline$\cdot 084$ & -1.027 & -013 & -955 \\
\hline $1 \cdot 153$ & $1 \cdot 860$ & $\cdot 930$ & $3 \cdot 943$ \\
\hline $52 \cdot 461$ & $3 \cdot 366$ & $3 \cdot 902$ & $59 \cdot 729$ \\
\hline
\end{tabular}

\begin{tabular}{|c|c|c|c|}
\hline$p a b$ & $p b b$ & $p b l$ & $p b s$ \\
\hline . & $\begin{array}{r}9 \cdot 522 \\
\cdot 002 \\
3 \cdot 371 \\
1 \cdot 428 \\
5 \cdot 760 \\
4 \cdot 688 \\
\cdot 200 \\
1 \cdot 153 \\
\cdot 087 \\
\cdot 389 \\
7 \cdot 081 \\
1 \cdot 153 \\
12 \cdot 482 \\
3 \cdot 000\end{array}$ & $\begin{array}{r}-4 \cdot 692 \\
.060 \\
-1 \cdot 905 \\
-\cdot 207 \\
-5 \cdot 040 \\
3 \cdot 750 \\
\cdot 320 \\
-.502 \\
-\cdot 750 \\
-\cdot 065 \\
-2 \cdot 142 \\
-.670 \\
\cdot 158 \\
1.500\end{array}$ & $\begin{array}{r}8 \cdot 073 \\
\cdot 176 \\
\cdot 986 \\
3.250 \\
4.656 \\
11 \cdot 738 \\
2 \cdot 100 \\
-1 \cdot 432 \\
-\cdot 016 \\
-.864 \\
-1.844 \\
-1.303 \\
11.613 \\
6.360\end{array}$ \\
\hline $3 \cdot 366$ & $50: 316$ & $-10 \cdot 188$ & $43 \cdot 493$ \\
\hline
\end{tabular}




\begin{tabular}{|c|c|c|c|}
\hline pal & $p b l$ & $p l l$ & pls \\
\hline & & $\begin{array}{r}2 \cdot 312 \\
2 \cdot 250 \\
\cdot 1 \cdot 080 \\
\cdot 030 \\
4 \cdot 410 \\
3 \cdot 000 \\
\cdot 512 \\
\cdot 219 \\
6 \cdot 483 \\
\cdot 011 \\
\cdot 648 \\
\cdot 389 \\
\cdot 002 \\
\cdot 750\end{array}$ & $\begin{array}{r}3 \cdot 978 \\
6 \cdot 600 \\
-\cdot 558 \\
-\cdot 471 \\
-4 \cdot 074 \\
9 \cdot 390 \\
3 \cdot 360 \\
\cdot 624 \\
\cdot 133 \\
\cdot 144 \\
.558 \\
\cdot 756 \\
\cdot 147 \\
3 \cdot 180\end{array}$ \\
\hline 3.902 & $-10 \cdot 188$ & $22 \cdot 096$ & $15 \cdot 811$ \\
\hline
\end{tabular}

The normal equations are therefore

$$
\begin{aligned}
52 \cdot 461 x+3 \cdot 366 y-3 \cdot 902 & =0, \\
3 \cdot 366 x+50 \cdot 316 y+10 \cdot 188 & =0,
\end{aligned}
$$

and may easily be solved by ordinary algebraic methods.

The quantity $[p l l]$ evaluated here, and $[l]$ in the previous example, will be required in evaluating the P.E.'s of the unknowns. It is convenient, though not necessary, to evaluate $[l l]$ or $[p l l]$ when forming the normal equations.

Example 3. A quantity $l$, of the form

$$
x \cos \theta+y \sin \theta+z=l,
$$

is observed for $\theta=5^{\circ}, 15^{\circ}, 25^{\circ}$, etc., up to $\theta=355^{\circ}$.

Form the normal equations.

With the previous notation, $a=\cos \theta, b=\sin \theta, c=1$. Since

$$
\cos ^{2} \theta=\sin ^{2}\left(90^{\circ}-\theta\right) \text {, }
$$

$$
\begin{aligned}
& {[a a]=\sum_{5^{\circ}}^{355^{\circ}} \cos ^{2} \theta=2 \underset{5^{\circ}}{\sum} \cos ^{2} \theta=4 \underset{5^{\circ}}{\mathbf{8}} \cos ^{2} \theta=18,} \\
& {[b b]=\sum_{5^{\circ}}^{855^{\circ}} \sin ^{2} \theta=18,} \\
& {[c c]=36,} \\
& {[a b]=\Sigma \cos \theta \sin \theta=0,} \\
& {[a c]=\Sigma \cos \theta=0,} \\
& {[b c]=\Sigma \sin \theta=0 .}
\end{aligned}
$$

Hence the normal equations are

$$
\begin{aligned}
& 18 x=[a l]=\Sigma l \cos \theta, \\
& 18 y=[b l]=\Sigma l \sin \theta, \\
& 36 z=[c l]=\Sigma l .
\end{aligned}
$$


Example 4. The instants of passage of twelve suecessive swings of a pendulum are observed. Find the period.

Let the observed times of the twelve successive passages be $t_{0}, t_{1}, t_{2}, \ldots t_{11}$. Let the true time of the first passage be $a_{0}$ and let the period be $T$. Then we have twelve observational equations,

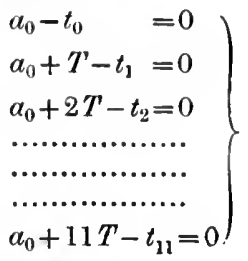

The normal equations are

$$
\begin{gathered}
12 a_{0}+(1+2+3+\ldots+11) T-\left(t_{0}+t_{1}+t_{2}+\ldots+t_{11}\right)=0 \\
(1+2+3+\ldots+11) a_{0}+\left(1^{2}+2^{2}+3^{2}+\ldots+11^{2}\right) T-\left(t_{1}+2 t_{2}+\ldots+11 t_{11}\right)=0 .
\end{gathered}
$$

If we substitute

$$
\begin{aligned}
& t_{0}+t_{1}+t_{2}+\ldots+t_{11}=s_{1}, \\
& t_{1}+2 t_{2}+\ldots+11 t_{11}=s_{2},
\end{aligned}
$$

the normal equations become

$$
\begin{aligned}
& 12 \alpha_{0}+66 T-s_{1}=0, \\
& 66 a_{0}+506 T-s_{2}=0 .
\end{aligned}
$$

Solving by the ordinary algebraie method, we find

$$
T=\frac{2 s_{2}-11 s_{1}}{286}, \quad a_{0}=\frac{23 s_{1}-3 s_{2}}{78}
$$

The following method of dealing with the above problem is entirely wrong, but affords an interesting example of the errors one is liable to commit.

Let the times be measured from the instant of the first passage, and let the times of the other passages be $t_{1}, t_{2}, \ldots t_{11}$. Then the observational equations are

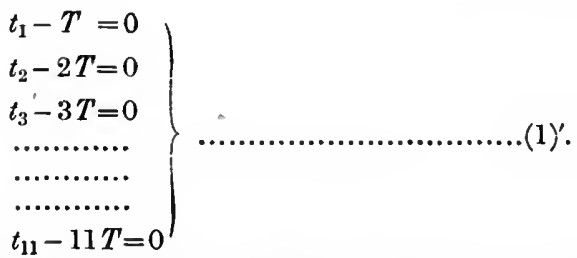

These equations are of equal weight, and the period is the value of $T$ which makes

$$
\Sigma\left(t_{s}-s T\right)^{2} \text { a minimum. }
$$

Differentiating with respect to $T$, we find

$$
T=\frac{t_{1}+2 t_{2}+\ldots+11 t_{11}}{1^{2}+2^{2}+\ldots+11^{2}}
$$


Or, if we return to the notation originally used, where the time of the first passage is $t_{0}$,

$$
T=\frac{s_{2}-(1+2+\ldots+11) t_{0}}{1^{2}+2^{2}+\ldots+11^{2}} .
$$

It should be noted that this result is entirely different from the one originally obtained in equation (2). This method of treatment is fallacious, because the error of noting the time of the first swing enters into each of the equations $(1)^{\prime}, t_{1}, t_{2}, \ldots t_{11}$, being the differences of two observed times. The method of least squares is only applicable to observational equations which are independent, and it is therefore not permissible to apply it to equations (1)'. The only correct method of dealing with this problem is to write down equations (1), and solve them by the method followed above.

Example 5. Form the observational equations for the rate and error of a sidereal clock, from the following set of observations. Derive the normal equations, and solve them.

\begin{tabular}{|c|c|c|}
\hline Star & Observed transit & R. A. \\
\hline$\eta$ Tauri & $3^{\mathrm{h}} \quad 38^{\mathrm{m}} \quad 7^{\mathrm{s}} \cdot 64$ & $8^{8} \cdot 08$ \\
\hline$A$ Tauri & $\begin{array}{lll}3 & 55 & 23 \\
\cdot & 16\end{array}$ & $23 \cdot 62$ \\
\hline$i$ Tauri & $\begin{array}{llll}4 & 53 & 41 & \cdot 19\end{array}$ & $41 \cdot 65$ \\
\hline$\beta$ Tauri & $\begin{array}{llll}5 & 16 & 20 & -56\end{array}$ & $21 \cdot 05$ \\
\hline
\end{tabular}

(The error of a clock at time $t$ is $a+b t$ where $a$ and $b$ are constants.)

Example 6. From the equations

$$
\begin{aligned}
& x=2 \text {, } \\
& x+y=3 \text {, } \\
& x-y+z=-1 \text {, } \\
& x-3 y+2 z=-2 \text {, }
\end{aligned}
$$

form the normal equations, and solve for $x, y, z$.

\section{Solution of the Normal Equations.}

In examples 2 and 5 above, there are only two unknowns in each case, so that there are only two normal equations; while in examples 1 and 6 there are three unknowns, but the coefficients of these unknowns in the normal equations are integral. In all four cases, therefore, the equations can be solved by the ordinary 
methods of elementary algebra. When the number of unknowns is greater than 2 , and the coefficients in the normal equations are not integral, the solution has to be carried out by one of the special methods discussed in the next chapter.

One of the difficulties that arise when we attempt to solve the equations in the haphazard way of ordinary algebra is that of determining the number of places of decimals to retain at each step. It is one of the merits of Gauss's method (to be discussed in the next chapter), that it prevents our getting into difficulties over this. 


\section{CHAPTER VI}

Evaluation OF the MOST PROBable VAlues OF THE UNKNOWNS, THEIR WEIGHTS AND PROBABLE ERRORS

\section{Gauss's Method of Substitution.}

For the sake of simplicity in writing, we shall suppose that there are three unknowns, $x, y, z$, but the method can be automatically extended to include any number of unknowns.

Let the normal equations be

$$
\left.\begin{array}{r}
{[a a] x+[a b] y+[a c] z-[a l]=0} \\
+[b b] y+[b c] z-[b l]=0 \\
+[c c] z-[c l]=0
\end{array}\right\}
$$

From the first equation we find

$$
x=-\frac{[a b]}{[a a]} y-\frac{[a c]}{[a a]} z+\frac{[a l]}{[a a]}
$$

Substituting this value in the second and third equations, we obtain the following equations:

$$
\left.\begin{array}{r}
{[b b 1] y+[b c 1] z-[b l 1]=0} \\
+[c c 1] z-[c l 1]=0
\end{array}\right\}
$$

where

$$
\left.\begin{array}{l}
{[b b 1]=[b b]-\frac{[a b][a b]}{[a a]}} \\
{[b c 1]=[b c]-\frac{[a b][a c]}{[a a]}}
\end{array}\right\}
$$

etc.

From the first equation in (iii),

$$
y=-\frac{[b c 1]}{[b b 1]} z+\frac{[b l 1]}{[b b 1]}
$$


Substituting this value in the second equation (iii), we find

$$
[c c 2] z-[c l 2]=0
$$

where

$$
\left.\begin{array}{l}
{[c c 2]=[c c 1]-\frac{[b c 1][b c 1]}{[b b 1]}} \\
{[c l 2]=[c l 1]-\frac{[b c 1][b l 1]}{[b b 1]}}
\end{array}\right\}
$$

Equation (vi) gives the value of $z$. Substituting this value for $z$, in equation (v) we evaluate $y$, and then by substitution for $y$ and $z$ in equation (ii) we obtain the value of $x$. The equations from which the values of the unknowns are deduced are here collected for convenience of reference.

$$
\begin{aligned}
& \left.\begin{array}{l}
z=\frac{[c l 2]}{\left[c c^{2}\right]} \\
y=-\frac{[b c 1]}{[b b 1]} z+\frac{[b l 1]}{[\overline{b b 1]}} \\
x=-\frac{[a b]}{[a a]} y-\frac{[a c]}{[a a]} z+\frac{[a l]}{[a a]}
\end{array}\right\}
\end{aligned}
$$

The solution for four unknowns is similar to the above, the work being carried through a further stage, involving the evaluation of $[d d 3]$, etc. In practice, the order of procedure is slightly varied from that shown above, but the method can be best understood by the actual solution of a set of normal equations.

\section{Checks in Computation.}

As the solution of a system of normal equations involves a considerable amount of arithmetic, it is advisable to check the correctness of the work at each stage.

a. In the first place, it can be shown that the leading coefficients [aa], [bb1], [cc2], etc., are all positive. Since [aa] is the sum of a number of squares, it must be positive. Again

Hence [ $b b 1]$ is positive.

$$
\begin{aligned}
{[a a][b b 1] } & =[a a][b b]-[a b][a b] \\
& =\Sigma\left(a_{r} b_{s}-a_{s} b_{r}\right)^{2} \\
& =\text { a positive quantity* } \\
& =[K K], \text { say. }
\end{aligned}
$$

\footnotetext{
* It follows that $[a a][b b]$ is greater than $[a b]^{2}$.
} 
Similarly $[a a][c c 1]=\Sigma\left(a_{r} c_{s}-a_{s} c_{r}\right)^{2}=[L L]$, say.

With this notation,

$$
\begin{aligned}
{[a a][b c 1] } & =[a a][b c]-[a c][a b] \\
& =\Sigma\left\{\left(a_{r} b_{s}-a_{s} b_{r}\right)\left(a_{r} c_{s}-a_{s} c_{r}\right)\right\} \\
& =[K L] .
\end{aligned}
$$

It follows from equation (vii) that

$$
\begin{aligned}
{[a a]^{2}[b b 1][c c 2] } & =[K K][L L]-[K L][K L] \\
& =\Sigma\left(K_{r} L_{s}-K_{s} L_{r}\right)^{2} \\
& =\text { a positive quantity. }
\end{aligned}
$$

Hence it follows that [cc2] is a positive quantity.

Since

$$
[a a][b b 1]=[K K], \quad[a a][b c 1]=[K L], \quad \text { etc., }
$$

it follows that the system of equations obtained after eliminating $x$ is similar to the original system of normal equations, in that all the coefficients in the leading diagonal are positive, while the other coefficients are symmetrical about this diagonal. The leading coefficient which results from eliminating two unknowns from this set of equations, will be of the same nature as [cc2]; i.e. it will be positive. This coefficient is [dd3]. This line of argument can be applied to any number of unknowns.

The calculation of [bb1], [cc2], etc., may in some measure be checked by the result deduced above, a negative value of such a coefficient indicating an error in computation.

B. A useful check on the calculations made in solving the normal equations, may be obtained by a simple extension of the checks on the formation of the normal equations (see $§ 37$ ). The formation of the equations is checked by means of quantities $[a s],[b s], \ldots,[l s]$. If we operate upon these quantities in the same way as upon $[a l],[b l]$, etc., we can form new quantities $[b s 1],[c s 1],[c s 2]$, etc. When there are only three unknowns, it is easily shown that

$$
\begin{aligned}
& {[b b 1]+[b c 1]+[b l 1]=[b s 1]} \\
& {[b c 1]+[c c 1]+[c l 1]=[c s 1] .}
\end{aligned}
$$

After the next stage of elimination we have

$$
[c c 2]+[c l 2]=[c s 2] \text {. }
$$


Similar relations will hold for any number of unknowns. A check of this kind is in general sufficient to detect any errors of computation. The only additional work involved in the carrying on of the check is the addition of a column to the tabular form in which the work is carried out.

$\gamma$. An additional check may be got by reversing the order of elimination of the unknowns, but this involves considerable labour.

$\delta$. When the unknowns have been evaluated, the substitution of their values in the observational equations yields the values of the residuals $v_{1}, v_{2}$, etc. A number of nseful checks on the work can be derived from these residuals. It has already been shown that

$$
[a v]=[b v]=[c v]=\ldots=0 .
$$

Any of these equations may be used as a check on the final results of solving the normal equations.

$\epsilon$. If the observational equations, written in the form

$$
a_{r} x+b_{r} y+c_{r} z-l_{r}=v_{r} \quad(r=1,2,3, \text { etc. })
$$

be multiplied by $v_{1}, v_{2}, v_{3}$, etc., and added, we obtain the equation

$$
-[v l]=[v v]
$$

since the coefficients of $x, y, z$, etc., vanish.

If the observational equations be multiplied by $l_{1}, l_{2}$, etc., and added, we obtain the equation

$$
[a l] x+[b l] y+[c l] z-[l l]=[l v]=-[v v] \ldots \ldots \ldots \text { (ix). }
$$

Now take equations (viii) and substitute in the last equation (ix) for $x, y, z$, in turn. Substituting for $x$, we obtain

$$
-[b l 1] y-[c l 1] z+[l l 1]=[v v] \text {. }
$$

Substituting for $y$, from the second of equations (viii),

$$
-[c l 2] z+[l l 2]=[v v] \text {. }
$$

Finally substituting for $z$, from the first of equations (viii),

$$
[l l 3]=[v v] \text {. }
$$

Hence

$$
[v v]=[l l 3]=[l l 2]-\frac{[c l 2]^{2}}{[c c 2]}
$$

or

$$
[v v]=[l l]-\frac{[a l]^{2}}{[a a]}-\frac{[b l 1]^{2}}{[b b 1]}-\frac{[c l 2]^{2}}{[c c 2]} \text {. }
$$


Any of the equations given above might be used as a check on the final result. Perhaps the most useful check is to compare the value of $[v v]$ obtained by squaring the calculated residuals, with the value of $[v v]$ obtained from the last equation. In any case the value of $[v v]$ will be required when we come to the problem of evaluating the probable errors of the adjusted values of the unknowns.

It should be noted that the last form for $[v v]$ is capable of immediate generalisation for any number of unknowns, say $m$. We then have

$$
[v v]=[l l m]=[l l]-\frac{[a l]^{2}}{[a a]}-\frac{[b l 1]^{2}}{[b b 1]}-\text { etc., }
$$

where there are $m$ terms after $[l l]$ on the right-hand side of the equation.

\section{Form of Solution.}

In any piece of work it is a decided advantage to follow a systematic method of solution, in which the evaluation of coefficients and the application of checks is to a large extent mechanical. Table A shows a convenient form of solution for four unknowns.

When there are only three unknowns, there will be no terms involving $d$, and only the first eleven lines in the table need be evaluated. When an arithmometer, or a table of products, is used, the solution can be worked out line by line as shown in the table. The form can be varied to suit individual tastes. The last column indicates how the various lines in the table are derived from the preceding lines. (Ch.) indicates the lines in which the checks are to be applied. When the work is to be done by the use of logarithmic tables, the order of computation need not be altered, but it is necessary to put in some additional lines containing logarithms. We shall solve by the use of logarithmic tables, following the order of Table $\mathrm{A}$ as closely as possible, the following set of normal equations :

$$
\begin{aligned}
& 153 \cdot 000 x+6 \cdot 285 y+2 \cdot 485 z-27 \cdot 831 w=22 \cdot 093 \\
& +8.989 y+4.037 z-0.426 w=-3.855 \quad 15.030 \\
& +23 \cdot 616 z-3 \cdot 504 w=-9 \cdot 952 \quad 16.682 \\
& +9 \cdot 080 w=7 \cdot 251 \quad-15 \cdot 430
\end{aligned}
$$




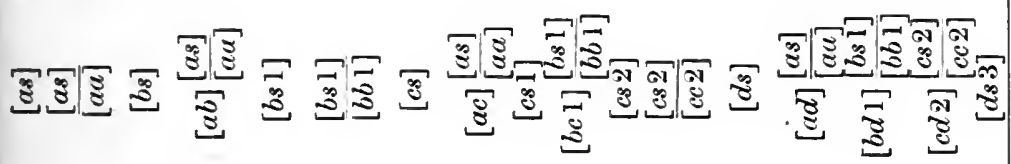

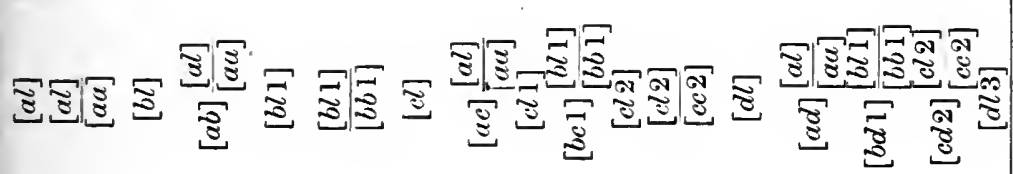

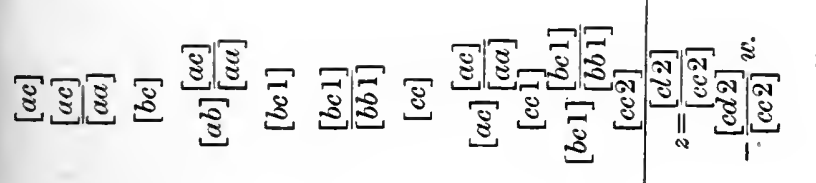

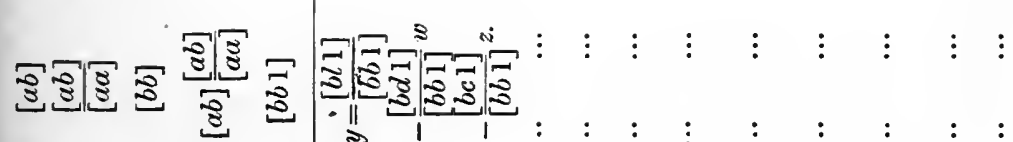

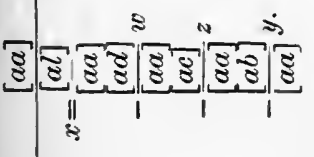

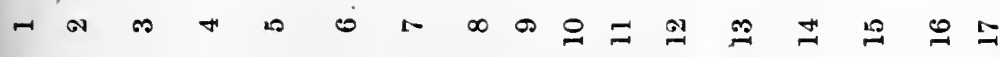


The solution of this set of equations is given in Table B. In this table the order of solution is that indicated in Table A. The last column indicates a comparison between this table and Table A, and the additional lines necessitated by the use of logarithmic tables are indicated by accented numbers in the first column.

A few points of procedure deserve notice. Line $1^{\prime}$ contains the logarithms of the quantities in line 1 . The logarithm of a negative quantity is expressed by writing down the logarithm of the quantity concerned, with $n$ prefixed, to indicate that we are dealing with a negative quantity. Thus

$$
\log -27 \cdot 831=n 1 \cdot 4445288,
$$

1.4445288 being $\log 27 \cdot 831$. Two $n$ 's added or subtracted annihilate one another. Line 2 is derived from line $1^{\prime}$ by subtracting the first term in line $\mathbf{l}^{\prime}$ from all the others. This can be conveniently done by writing the first term at the bottom of a piece of paper, and carrying the piece of paper along line $1^{\prime}$, so that this term may be subtracted in turn from each of the other terms. To derive line 4 from line 2 , we first add 0.7983053 to each term in line 2, and find from the tables the numbers corresponding to the sums. These numbers are written down in line 4. The remainder of the work follows the same method.

The final solution is

$$
\begin{aligned}
& x=0.727, \\
& y=-0.836, \\
& z=0.093, \\
& w=3.026 .
\end{aligned}
$$

\section{The Doolittle Method of Solution.}

A variant of Gauss's method of substitution, due to Mr Doolittle, of the U.S. Coast and Geodetic Survey*, yields a considerable gain in speed, and saves much labour by reducing the number of entries from the tables to a minimum. Take the case of three normal equations,

$$
\begin{array}{r}
{[a a] x+[a b] y+[a c] z=[a l]} \\
+[b b] y+[b c] z=[b l] \\
+[c c] z=[c l] .
\end{array}
$$

* Coast and Geodetic Survey, Report for 1878, Appendix 8, pp. 115-118. 


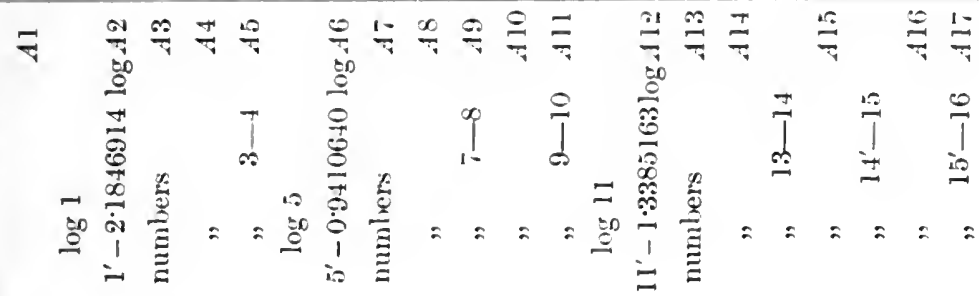

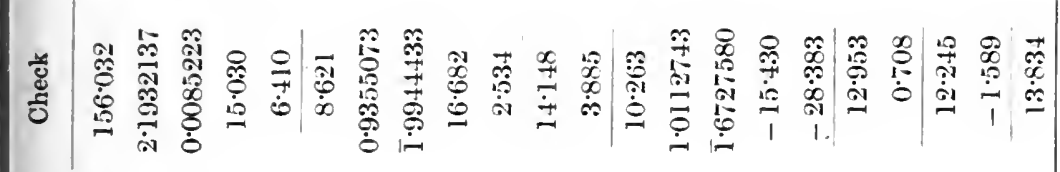

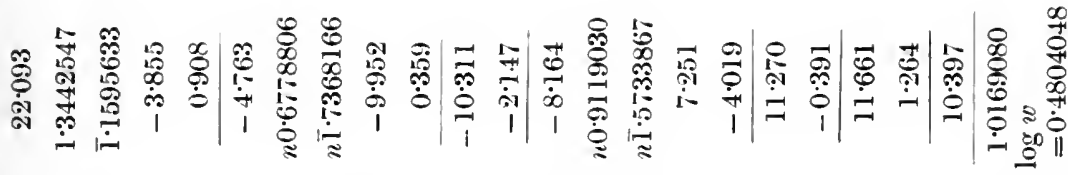

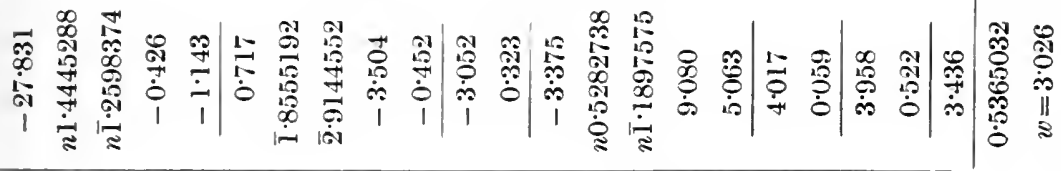

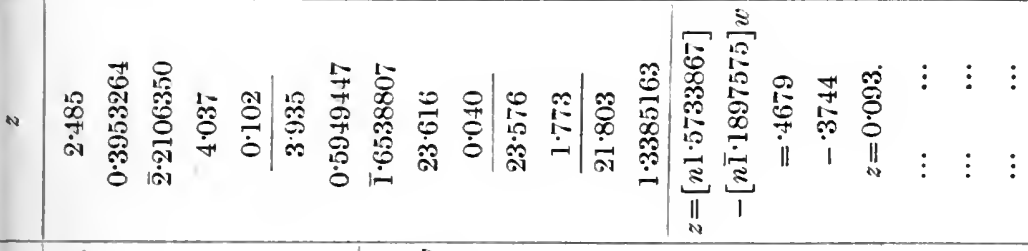

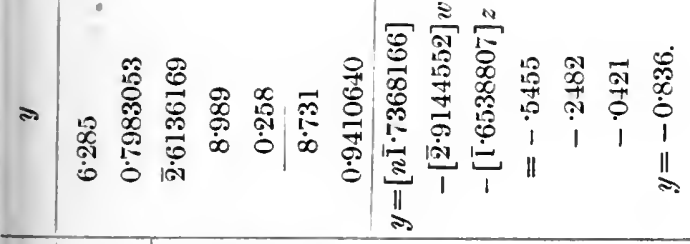

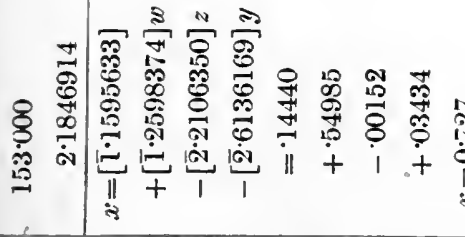

ー ニ ๙

B. 0 . 
The notation used in the solution is that of Gauss's method. The order of solution is shown in tables $\mathrm{C}$ and $\mathrm{D}$.

Table C.

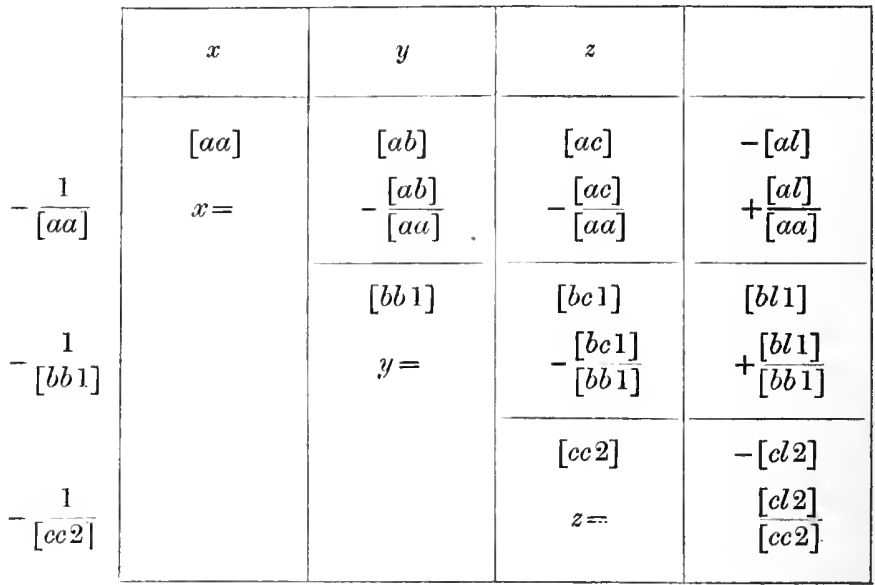

TABLE D.

\begin{tabular}{|c|c|c|}
\hline$y$ & $z$ & \\
\hline$[b b]$. & {$[b c]$} & $-[b l]$ \\
$-[a b] \frac{[a b]}{[a a]}$ & $-[a b] \frac{[a c]}{[a a]}$ & {$[a b] \frac{[a l]}{[a a]}$} \\
\hline & {$[c c]$} & $-[c l]$ \\
& $-[a c] \frac{[a c]}{[a a]}$ & {$[a c] \frac{[a l]}{[a a]}$} \\
& $-[b c 1] \frac{[b c 1]}{[b b 1]}$ & $\begin{array}{c}{[b c 1]} \\
{[b l 1]}\end{array}$ \\
\hline
\end{tabular}

The coefficients of the first normal equation are written in line 1, table C. The reciprocal of $[a a]$, with its sign changed, is written in the first column, and all the other quantities in line $\mathbf{1}$ are multiplied by it, the results being entered in line 2 . Line 2 then gives $x$ as an explicit function of $y$ and $z$. The coefficients of the second normal equation are written in line 1 , table $\mathrm{D}$. Line 2, table $\mathrm{C}$, is now multiplied by $[a b]$, and the results entered in 
table $\mathrm{D}$, line 2. The sum of lines 1 and 2 , table $\mathrm{D}$ ), is entered in table $\mathrm{C}$, line 3 . The reciprocal of $[b b 1]$ is written in the first column of table $\mathrm{C}$, and all the other quantities in line 3 are multiplied by this reciprocal, the results being entered in line 4. This line gives $y$ as an explicit function of $z$. Next, the coefficients of the third normal equation are entered in line 3 of table $\mathrm{D}$. 'The terms in lines 2 and 4 of table C, beginning only at the $z$ terms, are multiplied by $[a c]$ and $[b c 1]$ respectively, and the results entered in table $\mathrm{D}$, lines 4 and 5 . The sum of lines 3,4 and 5 of table $\mathrm{D}$ is entered in table $\mathrm{C}$, line 5 . Line 6 , table $\mathrm{C}$, is obtained by first writing down the reciprocal of [ $c c 2$ ], with its sign changed, and multiplying the other term of line 5 by this residual. Line 6 gives the value of $z$. The values of $y$ and $x$ are obtained by successive substitution in lines 4 and 2 of table $\mathrm{C}$.

This method of solution can be easily extended for any number of unknowns. Its advantage over the older method, due to the reduction of entries, is even greater for larger numbers of unknowns than for three unknowns as discussed above. .In practice it is convenient to make tables $C$ and $D$ on separate sheets of paper, and to fold table $\mathrm{C}$ along alternate lines, to facilitate the carrying of the numbers of table $\mathrm{C}$ to where they are required in table $\mathrm{D}$.

\section{Solution by the Method of Determinants*.}

The solution of the normal equations

$$
\begin{aligned}
{[a a] x+[a b] y+[a c] z } & =[a l] \\
+[b b] y+[b c] z & =[b l] \\
+[c c] z & =[c l]
\end{aligned}
$$

may be immediately written down in determinantal form

$$
x=\frac{1}{D}\left|\begin{array}{ccc}
{[a l]} & {[a b]} & {[a c]} \\
{[b l]} & {[b b]} & {[b c]} \\
{[c l]} & {[b c]} & {[c c]}
\end{array}\right|, \quad y=\frac{1}{D}\left|\begin{array}{ccc}
{[a c]} & {[a l]} & {[a c]} \\
{[a b]} & {[b l]} & {[b c]} \\
{[a c]} & {[c l]} & {[c c]}
\end{array}\right|,
$$

where

$$
D=\left|\begin{array}{ccc}
{[a a]} & {[a b]} & {[a c]} \\
{[a b]} & {[b b]} & {[b c]} \\
{[a c]} & {[b c]} & {[c c]}
\end{array}\right| \text {. }
$$

* This method is not a very practical one in general. It is given here because it is useful in the development of the theory. 
'These results may be extended to any number of unknowns. The determinant $D$, which is symmetrical about the leading diagonal, contains as many rows and columns as there are unknowns.

The above solution leads to a form for calculating [vv] which is occasionally useful. It has already been shown (page 93) that

$$
\begin{aligned}
& {[v v]=[l l]-[a l] x-[b l] y-[c l] z \text {. }} \\
& \therefore D[v v]=[l l] \times\left|\begin{array}{lll}
{[a a]} & {[a b]} & {[a c]} \\
{[a b]} & {[b b]} & {[b c]} \\
{[a c]} & {[b c]} & {[c c]}
\end{array}\right|+[a b]\left|\begin{array}{ccc}
{[a b]} & {[a c]} & {[a l]} \\
{[b b]} & {[b c]} & {[b l]} \\
{[b c]} & {[c c]} & {[c l]}
\end{array}\right| \\
& -[b l]\left|\begin{array}{ccc}
{[a a]} & {[a c]} & {[a l]} \\
{[a b]} & {[b c]} & {[b l]} \\
{[a c]} & {[c c]} & {[c l]}
\end{array}\right|+[c l]\left|\begin{array}{ccc}
{[a a]} & {[a b]} & {[a l]} \\
{[a b]} & {[b b]} & {[b l]} \\
{[a c]} & {[b c]} & {[c l]}
\end{array}\right| \text {. } \\
& \therefore \quad D[v v]=\left|\begin{array}{llll}
{[a a]} & {[a b]} & {[a c]} & {[a l]} \\
{[a b]} & {[b b]} & {[b c]} & {[b l]} \\
{[a c]} & {[b c]} & {[c c]} & {[c l]} \\
{[a l]} & {[b l]} & {[c l]} & {[l l]}
\end{array}\right| .
\end{aligned}
$$

This is a determinant formed from $D$ by the addition of a fresh row and a fresh column containing the $l$ terms.

\section{Probable Error of an Observational Equation of} Unit Weight, when there are $n$ Observations involving m Independent Unknowns.

If the observations be not all of the same weight, each observational equation is first multiplied by the square root of its weight, so as to reduce all the residuals to unit weight. Let the system of residuals, thus reduced to unit weight, be $v_{1}, v_{2}$, etc: If the error law followed by the residuals be

$$
\frac{h}{\sqrt{ } \pi} e^{-h^{2} \Delta^{2}}
$$

then the a priori probability of the given system of residuals $v_{1}, v_{2}$, etc., is

$$
C h^{n} e^{-h^{2}[v v]},
$$

where $C$ is a numerical constant (cf. $\S 14$ ). - This probability may be written

$$
C h^{n} e^{-h^{2} \Sigma\left(a_{r} x+b_{r} y+c_{r} z+\ldots-l_{r}\right)^{2}}
$$


But $x, y$, etc., are all unknown and inclependent, and may, as far as we know, take any values between $-\infty$ and $+\infty$. The complete probability is therefore got by integrating the last expression between limits $\pm \infty$ for each of the unknowns. The probability then becomes

$$
C \int_{\substack{\infty \\ m \text { Integrations }}}^{+\infty} \int_{-\infty}^{+\infty} \ldots \int_{-\infty}^{+\infty} h^{n} e^{-h^{2} \Sigma\left(a_{,}, x+b_{r} y+\ldots-l_{r}\right)^{2}} d x d y d z \ldots .
$$

In order to integrate with respect to $x$, we write the coefficient of $-h^{2}$ in the exponential term, in the form

$$
P+(Q x+R)^{2}
$$

where $Q$ is a numerical factor, and $P$ and $R$ are functions of $y, z$, ete.

$$
\begin{aligned}
\int_{-\infty}^{+\infty} h e^{-h^{2}\left\{P+(Q x+R)^{2}\right\}} d x & =h e^{-h^{2} P} \int_{-\infty}^{+\infty} e^{-h^{2}(Q x+R)^{2}} d x \\
& =\frac{\sqrt{ } \pi}{Q} \cdot e^{-h^{2} P} .
\end{aligned}
$$

The factor $\frac{\sqrt{ } \pi}{Q}$ is a numerical factor which depends only on the values of the coefficients in the observational equations. We may regard it as being absorbed in the constant $C$.

The expression for the probability, with $x$ eliminated, becomes

$$
C \int_{\substack{-\infty \\(m-1)}}^{+\infty} \int_{\substack{-\infty \\ \text { Integrations }}}^{+\infty} \ldots . . \int_{-\infty}^{+\infty} h^{n-1} e^{-h^{2} P} d y d z \ldots
$$

Here $P$ is the quadratic function of $y, z$, etc., which results from

$$
\Sigma\left(a_{r} x+b_{r} y+\ldots-l_{r}\right)^{2}
$$

when $x$ is so chosen as to make this last expression a minimum. It follows that after $m$ integrations, the probability becomes

$$
C h^{n-m} e^{-h^{2} T}
$$

where $C$ is a constant, and $T$ is the value of $\Sigma\left(a_{r} x+b_{r} y+\ldots-l_{r}\right)^{2}$. which results from giving $x, y, z$, etc., the values which make this sum a minimum. But these are the values of $x, y, z$, etc., which 
are determined by the ordinary least square solution, using the normal equations. It follows that

$$
T=[v v]
$$

The total probability of the given system of residuals is thus

$$
C h^{n-m} e^{-h^{2}[v v]} \text {. }
$$

The value of the parameter $h$ which determines the scale of the error curve has to be selected so as to make the probability of the given system of residuals a maximum. Taking logarithms, and differentiating with respect to $h$, we find

or

$$
\begin{gathered}
\frac{n-m}{h}-2 h[v v]=0, \\
h=\sqrt{\frac{n-m}{2[v v]}} .
\end{gathered}
$$

The M.S.E. $\mu$ of an observation of unit weight is thus

$$
\mu=\sqrt{\frac{[v v]}{n-m}},
$$

and the P.E. $r$ of an observation of unit weight, or an observational equation of unit weight, is given by*

$$
r=0.6745 \sqrt{\frac{[v v]}{n-m}} .
$$

The quantity $[v v]$ may be evaluated in a number of ways. The obvious method is to calculate it from the actual residuals, and since it is generally advisable to test the character of the work by evaluating the residuals and considering their magnitude, this is perhaps the most satisfactory method. But it is also possible to calculate $[v v]$ from any of the relations shown below.

$$
\begin{aligned}
& {[v v]=-[v l],} \\
& {[v v]=[l l]-[a l] x-[b l] y-[c l] z-\text { etc. }} \\
& {[v v]=[l l]-\frac{[a l]^{2}}{[a a]}-\frac{[b l 1]^{2}}{[b b 1]}-\frac{[c l 2]^{2}}{[c c 2]}-\text { etc. }}
\end{aligned}
$$

Or any one of these relations may be used as a check upon the value obtained by squaring the residuals. of weight $p$.

* Or $r=0.6745 \sqrt{\frac{[p v v]}{n-m}}$, where $v$ is the residual in an observational equation 


\section{Evaluation of Probable Errors of the Unknowns.}

Since the normal equations are linear in $x, y, z$, etc., and also in $l_{1}, l_{2}$, ete., we may write

$$
\begin{aligned}
& x=\alpha_{1} l_{1}+\alpha_{2} l_{2}+\text { etc. } \\
& y=\beta_{1} l_{1}+\beta_{2} l_{2}+\text { etc. }
\end{aligned}
$$

where $\alpha_{1}, \alpha_{2}, \ldots, \beta_{1}, \beta_{2}, \ldots$, etc., are functions of the coefficients $a, b, c$.

The P.E. of an observational equation of unit weight, or the P.E. of each of the $l$ 's, is the quantity $r$ which has already been deduced above. If $r_{x}, r_{y}$, etc., be the P.E.'s of the unknowns, it follows that

$$
r_{x}^{2}=r^{2}[\alpha \alpha], \quad r_{y}^{2}=r^{2}[\beta \beta], \quad \text { etc. }
$$

It is therefore necessary to evaluate $[\alpha \alpha],[\beta \beta]$, etc. The reciprocals of these sums may be regarded as the weights of $x, y$, etc. Calling these $p_{x}, p_{y}$, etc., we have the relations

$$
p_{x}=\frac{1}{[\alpha \alpha]}, \quad p_{x} r_{x}^{2}=r^{2} .
$$

Returning to the solution of the normal equations by determinants, we may write

$$
x=\frac{1}{D}\left|\begin{array}{lll}
{[a l]} & {[a b]} & {[a c]} \\
{[b l]} & {[b b]} & {[b c]} \\
{[c l]} & {[b c]} & {[c c]}
\end{array}\right| \equiv \alpha_{1} l_{1}+\alpha_{2} l_{2}+\ldots
$$

Collecting the terms in $l_{1}$ in the determinant, we have the relation

$$
D \alpha_{1}=\left|\begin{array}{lll}
a_{1} & {[a b]} & {[a c]} \\
b_{1} & {[b b]} & {[b c]} \\
c_{1} & {[b c]} & {[c c]}
\end{array}\right|=a_{1} A+b_{1} B+c_{1} C,
$$

where $A, B, C$ are the minors of $a_{1}, b_{1}, c_{1}$, in the determinant. Squaring this expression, we may write the result as

$$
\begin{aligned}
D^{2} \alpha_{1}^{2}= & A\left(a_{1} a_{1} A+a_{1} b_{1} B+a_{1} c_{1} C\right) \\
& +B\left(a_{1} b_{1} A+b_{1} b_{1} B+b_{1} c_{1} C\right) \\
& +C\left(a_{1} c_{1} A+b_{1} c_{1} B+c_{1} c_{1} C\right) .
\end{aligned}
$$


Summing for all possible suffixes we find

$$
\begin{aligned}
D^{2}[a \alpha]=A & \{[a a] A+[a b] B+[a c] C\} \\
& +B\{[a b] A+[b b] B+[b c] C\} \\
& +C\{[a c] A+[b c] B+[c c] C\} \\
=A & \{[a a] A+[a b] B+[a c] C\}=A D .
\end{aligned}
$$

For if the second and third brackets be written in the form of determinants, two columns of each determinant would be the same, and so their values would be zero.

$$
\text { Hence } \quad[a \alpha]=\frac{A}{D}=\left|\begin{array}{lll}
1 & {[a b]} & {[a c]} \\
0 & {[b b]} & {[b c]} \\
0 & {[b c]} & {[c c]}
\end{array}\right| \div D .
$$

Comparing this with the value of $x$ deduced above, we see that $[\alpha \alpha]$ is the value of $x$ which we should deduce if we put

$$
[a l]=1, \quad[b l]=[c l]=0 .
$$

Similarly $[\beta \beta]$ is the value of $y$ obtained when we put

$$
[a l]=[c l]=0 \text { and }[b l]=1 \text {. }
$$

where $B, F$, and $G$ are the minors of $a_{1}, b_{1}, c_{1}$, in the determinant. $B$ is the same as the $B$ given above.

We thus have $D \alpha=a_{1} A+b_{1} B+c_{1} C$.

$$
\begin{aligned}
D \beta= & a_{1} B+b_{1} F+c_{1} G . \\
\therefore \quad D^{2} \alpha \beta= & A\left\{a_{1} a_{1} B+a_{1} b_{1} F+a_{1} c_{1} G\right\} \\
& +B\left\{a_{1} b_{1} B+b_{1} b_{1} F+b_{1} c_{1} G\right\} \\
& +C\left\{a_{1} c_{1} B+b_{1} c_{1} F+c_{1} c_{1} G\right\} \\
D^{2}[\alpha \beta]= & A\{[a a] B+[a b] F+[a c] G\} \\
& +B\{[a b] B+[b b] F+[b c] G\} \\
& +C\{[a c] B+[b c] F+[c c] G\} \\
= & D \cdot B,
\end{aligned}
$$


the first and last brackets being zero, as can easily be shown by writing them in determinant form. It follows that

$$
D[\alpha \beta]=B=\left|\begin{array}{ccc}
{[a c]} & 1 & {[a c]} \\
{[a b]} & 0 & {[b c]} \\
{[a c]} & 0 & {[c \dot{c}]}
\end{array}\right|=\left|\begin{array}{ccc}
0 & {[a b]} & {[a c]} \\
1 & {[b b]} & {[b c]} \\
0 & {[b c]} & {[c c]}
\end{array}\right|,
$$

$[\alpha \beta]$ is therefore the value of $y$ obtained when

$$
[a l]=1, \quad[b l]=[c l]=0,
$$

or the value of $x$ obtained when

$$
[a l]=[c l]=0, \quad[b l]=1 .
$$

\section{Evaluation of the Weights of $x, y, z$, etc.}

The weight of the unknown which is first evaluated in the Gauss solution can be immediately deduced. If in the equations of $\S 41$, we put

$$
[a l]=[b l]=0 \text { and }[c l]=1 \text {, }
$$

then

$$
[b l 1]=0 \text {, and }[c l 2]=1 \text {. }
$$

Equation (vi) then yields $[c c 2][\gamma \gamma]=1$,

or

$$
p_{z}=\frac{1}{[\gamma \gamma]}=[c c 2] \text {. }
$$

The quantity [cc2] is one of the quantities evaluated in the process of solving the normal equations. In the general case where there are $m$ unknowns, the weight of the last unknown in the order of elimination is the coefficient of that unknown in the last elimination equation. Thus in table $\mathrm{A}$, page 95 , the weight of $w$ is given by

$$
p_{w}=[d d 3] .
$$

The weight of any unknown might be evaluated by making that particular unknown the last in the order of elimination. If the normal equations involve a large number of unknowns, of which it is only necessary to find the weights of a few, it is a considerable advantage to eliminate last the unknowns whose weights are required.

The following special cases should be particularly noted. 
(a) When there are only two unknowns, $x$ and $y$.

With the usual notation

And similarly,

$$
p_{y}=[b b 1]=\frac{[a a] \cdot[b b]-[a b] \cdot[a b]}{[a a]} .
$$

(b) When there are three unknowns, $x, y, z$.

It has been shown in $\S 47$, that

Similarly

$$
p_{x}=\frac{1}{[\alpha \alpha]}=\frac{D}{A}=\frac{D}{[b b][c c]-[b c][b c]} .
$$

$$
p_{y}=\frac{D}{[c c][a a]-[a c][a c]}, \quad p_{z}=\frac{D}{[a a][b b]-[a b][a b]},
$$

where $D$ is the determinant formed by the coefficients of the normal equations. It may also be written

$D=[a a][b b][c c]+2[a b][b c][c a]-[a a][b c]^{2}-[b b][c a]^{2}-[c c][a b]^{2}$.

When the normal equations contain only integral coefficients, it is generally simpler to solve them by the methods of ordinary algebra, and to calculate the weights by means of the above equations.

(c) For any number of unknowns, it is easy to write down the weight of the last but one of the unknowns.

Take the case of four unknowns eliminated in the order $x, y, z, w$. Then the weight of $w$ is [dd3]. If we re-modelled our solution, and eliminated in the order $x, y, w, z$, the auxiliary quantities evaluated in the solution would remain unaltered as far as affix 2. The normal equations remaining after eliminating $x$ and $y$ are

$$
\begin{aligned}
& {[d d 2] w+[\operatorname{cd} 2] z-[d l 2]=0,} \\
& {[c d 2] w+[c c 2] z-[c l 2]=0 .}
\end{aligned}
$$

From the first of these,

$$
w=-\frac{[c d 2]}{[\overline{d d 2}]} z+\frac{[d l 2]}{[\overline{d d 2}]}
$$


Substituting this in the last equation, we find

$$
\begin{aligned}
p_{z} & =\text { coefficient of } z \text { in the final equation } \\
& =[c c 2]-[c d 2] \frac{[c d 2]}{[d d 2]} \\
& =\frac{[c c 2]}{[d d 2]}\left\{[d d 2]-\frac{[c d 2][c d 2]}{[c c 2]}\right\} . \\
p_{z} & =\frac{[c c 2][d d 3]}{[d d 2]} .
\end{aligned}
$$

Thus $p_{z}$ is easily determined. For [cc2] is the last coefficient in the [cc] column, [dd.3] the last coefficient in the $[d d]$ column, and [dd2] the last coefficient but one in the $[d d]$ column.

Thus in the example worked in table $\mathrm{B}$, page $97, p_{w}=3 \cdot 436$, and

$$
p_{z}=\frac{21 \cdot 803 \times 3.436}{3.958}
$$

\section{EXAMPLES.}

1. Continuation of Ex. 1, page 82 .

To find the weights and P.E.'s of $x, y, z$. Using the formulae of case (b) above, we find

$$
\begin{aligned}
& D=27 \times 15 \times 54-27-54 \times 6^{2}=19899, \\
& p_{x}=\frac{19899}{15 \times 54-1}=24 \cdot 6, \\
& p_{y}=\frac{19899}{27 \times 54}=13 \cdot 7, \\
& p_{z}=\frac{19899}{27 \times 15-36}=54.0 .
\end{aligned}
$$

Substituting the derived values of the unknowns in the observational coriations, we obtain the following residuals,

$$
+\cdot 24+\cdot 09-\cdot 11-\cdot 06
$$

The P.E. of a single observational equation is

or

$$
0.6745 \sqrt{(\cdot 24)^{2}+(\cdot 09)^{2}+(\cdot 11)^{2}+(\cdot 06)^{2}},
$$

$0 \cdot 19$.

$$
\begin{aligned}
\therefore r_{x} & =\frac{0 \cdot 19}{\sqrt{24 \cdot 6}}=0.038, \\
r_{y} & =\frac{0 \cdot 19}{\sqrt{13 \cdot 7}}=0.052, \\
r_{z} & =\frac{0.19}{\sqrt{54 \cdot 0}}=0.026 .
\end{aligned}
$$


2. Solve the normal equations of Ex. 2, page 84, evaluate the weights of the unknowns $x$ and $y$, and from the formula

$$
[p v v]=[p l l]-\frac{[p a l]^{2}}{[p a \alpha]}-\frac{[p b l 1]^{2}}{[p b b 1]}
$$

find the P.E. of an obserrational equation of unit weight, and the P.E.'s of $x$ and $y$.

3. Verify the result of the last example by evaluating [ $p v v]$ from the residuals.

4. Prove that in the case of four unknowns $x, y, z, w$, eliminated in this order, the weight of $y$ is

$$
p_{y}=\frac{[b b 1][c c 2][d d 3]}{[c c 1][d d 2]_{b}}
$$

where all the auxiliaries have the usual meaning except $[d d 2]_{b}$, which is the value of $[d d 2]$ when the unknowns are eliminated in the order $x, z, w, y$.

The product $[a \alpha][b b 1][c c 2][d d 3]$ is the common denominator of $x, y, z, w$, when the expressions for the unknowns are reduced to the same denominator, The value of the product is therefore independent of the order of elimination.

$$
\therefore[a \alpha][b b 1][c c 2][d d 3]=[\alpha a][c c 1][d d 2]_{b}[b b 3] \text {, }
$$

whence the result immediately follows.

Deduce from table $\mathrm{B}$ the weight of $y$.

5. Show for the ease of three unknowns, that the weights of the unknowns must always be positive.

6. In Ex. 3, page 86, find the weights of the unknowns.

7. In the same example show that

$$
[v v]=[l l]-36 z^{2}-18\left(x^{2}+y^{2}\right) \text {. }
$$

Write down the equations of condition in the form

$$
v_{r}+l_{r}=x \cos \theta+y \sin \theta+z \text {. }
$$

Multiply by $l_{r}$ and add, remembering the relation

$$
[v v]=-[v l] \text {. }
$$

\section{An Alternative Method of finding the Weights of the Unknowns.}

It has been shown that the weight of $x$ is the value of $x$ derived by solving a new set of normal equations in which

$$
[a l]=1, \quad[b l]=[c l]=\ldots=0 .
$$

The weight of each unknown can in fact be derived by solving the appropriate set of normal equations. In practice the weights can be conveniently found by combining the solution of all these subsidiary sets of normal equations with the ordinary solution for the 
unknowns. For the case of three unknowns, three arditional columns are added to the table of solution, with $1,0,0 ; 0,1,0$; and $0,0,1$; written in positions corresponding to $[a l],[b l]$ and $[c l]$. If these columns be hearled $R, S$ and $T$ respectively, the values of $z$ derived in these columns are

$$
[\alpha \gamma],[\beta \gamma],[\gamma \gamma] .
$$

The derivation of $[\alpha \alpha]$ and $[\beta \beta]$ follows quite simply from these. The method can be most clearly understood from the working of an actual example.

The following set of normal equations were derived by H. N. Russell in some work on stellar parallax :

$$
\begin{aligned}
8 \cdot 000 x+4 \cdot 106 y+0.698 z & =-2588 \\
+3.989 y+0.051 z & =-1441 \\
+4.613 z & =1349 .
\end{aligned}
$$

The work of solution is considerably simplified by changing the unit of the absolute terms to 1000 times its original value. For, as the equations stand, the check sum for the first equation is 2545.196; while in the equation obtained by our suggested change of unit, the check sum is $10 \cdot 246$, containing five significant figures instead of seven.

In writing the absolute terms in the table of solution, we change the unit, so that they become $-2 \cdot 588,-1 \cdot 441,1349$.

The solution is given on page 110. The last column indicates a comparison with the order of solution in table $A$, page 95 . The last column but one shows how each line is derived from those which precede it. The first three lines in the check column give $[a s]+1,[b s]+1,[c s]+1$.

The work of solution was carried out by means of a Brunsviga calculator. Division of a whole line by any quantity, as for example, the division of line 8 by [bb1], i.e. by 1.8826 , was carried out by multiplying the whole line by the reciprocal of [bb1].

The last line of the table gives the values of $z,[\alpha \gamma],[\beta \gamma],[\gamma \gamma]$. $z=0.3453,[\alpha \gamma]=-0.0357,[\beta \gamma]=0.0362,[\gamma \gamma]=0.2221$.

From line 9,

$$
\begin{aligned}
y & =0.1627 z-0.0616=-0.0044 . \\
{[\beta \beta] } & =0.1627[\beta \gamma]+0.5312=0.5371 . \\
{[\alpha \beta] } & =0.1627[\alpha \gamma]-0.2726=-0.2784 .
\end{aligned}
$$




\begin{tabular}{|c|c|c|c|c|c|c|c|c|c|c|c|c|}
\hline$T$ & - & $\therefore$ & $1-$ & 01 & + & 10 & $\infty$ & $D$ & 0 & $\stackrel{ }{2}$ & $\exists$ & $\stackrel{\sim}{\sim}$ \\
\hline \multirow{3}{*}{ 苞 } & \multirow[t]{2}{*}{1} & \multirow[t]{2}{*}{1} & \multirow[t]{2}{*}{1} & $\frac{\widetilde{E}}{*}$ & $\frac{\sqrt[\vec{E}]{*}}{\times}$ & $\stackrel{\Omega}{5}$ & $\frac{\bar{\Xi}}{x}$ & $\stackrel{E}{E}$ & $\underset{8}{7}$ & $\frac{\vec{J}}{\vec{J}}$ & $\underset{1}{\stackrel{O}{g}}$ & $\frac{\text { 胥 }}{\stackrel{8}{*}}$ \\
\hline & & & & 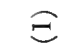 & 王 & ล & & $\widehat{\vartheta}$ & $\widehat{\infty}$ & $\widehat{\widehat{\theta}}$ & $\widehat{\infty}$ & $\widehat{\Xi}$ \\
\hline & 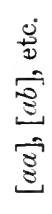 & $\begin{array}{l}0 \\
0 \\
0 \\
0 \\
0 \\
0 \\
0\end{array}$ & 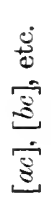 & 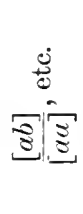 & $\frac{\sqrt[3]{0}}{\frac{5}{3}}$ & $\begin{array}{l}0 \\
0 \\
0 \\
0 \\
0 \\
0\end{array}$ & 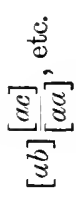 & 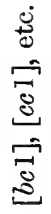 & 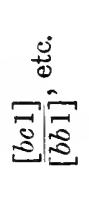 & 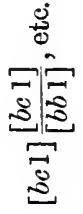 & 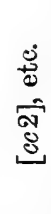 & 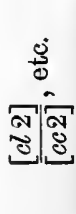 \\
\hline 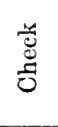 & 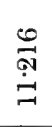 & $\underset{i}{\stackrel{R}{0}}$ & $\underset{i}{\underset{i}{*}}$ & 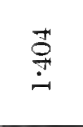 & $\underset{:}{\stackrel{\infty}{0}}$ & 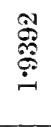 & $\begin{array}{l}8 \\
8 \\
\$ \\
0 \\
0\end{array}$ & 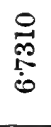 & 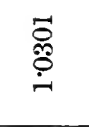 & 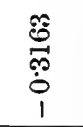 & $\begin{array}{l}\stackrel{9}{*} \\
\stackrel{+}{0} \\
\stackrel{0}{i}\end{array}$ & 1 \\
\hline$E$ & 0 & 0 & - & 1 & 1 & 1 & 1 & $r$ & 1 & 1 & - & สิส \\
\hline$\sigma_{2}$ & 0 & $r$ & 0 & 1 & 1 & - & 1 & 1 & 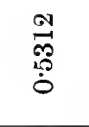 & $\frac{\overrightarrow{\tilde{O}}}{\dot{0}}$ & $\overrightarrow{\mathscr{\theta}}$ & $\begin{array}{l}\text { 용 } \\
\mathscr{O} \\
\dot{0}\end{array}$ \\
\hline 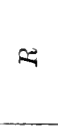 & - & 0 & 0 & $\stackrel{29}{3}$ & $\stackrel{9}{\stackrel{9}{: 0}}$ & $\begin{array}{l}\stackrel{0}{*} \\
\dot{0} \\
\dot{0} \\
1\end{array}$ & $\begin{array}{l}\stackrel{9}{1} \\
\infty \\
0 \\
0\end{array}$ & 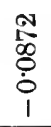 & $\begin{array}{l}\stackrel{0}{N} \\
\text { ज़ } \\
0 \\
1\end{array}$ & $\begin{array}{l}\stackrel{1}{0} \\
\stackrel{0}{0} \\
\dot{0}\end{array}$ & $\begin{array}{l}\text { Oे } \\
\stackrel{0}{0} \\
\dot{0} \\
1\end{array}$ & $\begin{array}{l}10 \\
\text { \% } \\
0 \\
1\end{array}$ \\
\hline & $\begin{array}{l}\infty \\
\infty \\
0 \\
\stackrel{0}{\omega} \\
i \\
1\end{array}$ & $\frac{9}{+5}$ & $\stackrel{\mathscr{P}}{\stackrel{9}{\sim}}$ & $\begin{array}{l}000 \\
\stackrel{0}{0} \\
0 \\
0 \\
1\end{array}$ & 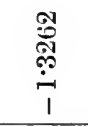 & \begin{tabular}{l}
$\infty$ \\
$\stackrel{\infty}{a}$ \\
\hdashline \\
1 \\
1
\end{tabular} & 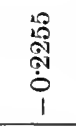 & 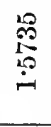 & $\begin{array}{l}0 \\
0 \\
\varnothing \\
0 \\
1\end{array}$ & 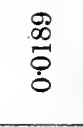 & 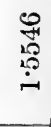 & 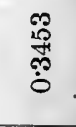 \\
\hline N & $\begin{array}{l}\infty \\
\stackrel{8}{0} \\
\stackrel{0}{0}\end{array}$ & $\begin{array}{l}\overline{20} \\
\stackrel{0}{0}\end{array}$ & 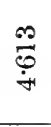 & '0 & \begin{tabular}{l}
$\stackrel{9}{1}$ \\
$\substack{10 \\
\hdashline \\
0}$
\end{tabular} & 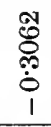 & 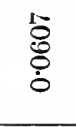 & 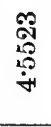 & $\begin{array}{l}\text { 今్ } \\
\stackrel{0}{0} \\
1 \\
1\end{array}$ & 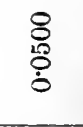 & 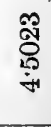 & $\begin{array}{l}\vdots \\
\vdots\end{array}$ \\
\hline$\therefore$ & 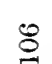 & $\not 8$ & $\overline{5}$ & $\stackrel{9}{\ddot{\theta}}$ & ت艹 & \%ั: & $\vec{\infty}$ & 동 & $\vdots$ & $\vdots$ & $\vdots$ & $\vdots$ \\
\hline$\infty$ & $\overline{\dot{j}}$ & $\dot{\dot{m}}$ & $\dot{0}$ & 0 & $\overrightarrow{\dot{\theta}}$ & $\stackrel{\infty}{-}$ & $\ddot{\theta}$ & î & $\vdots$ & $\vdots$ & $\vdots$ & $\vdots$ \\
\hline 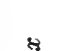 & 8 & 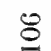 & $\infty$ & $\vdots$ & $\vdots$ & $\vdots$ & $\vdots$ & $\vdots$ & $\vdots$ & $\vdots$ & $\vdots$ & $\vdots$ \\
\hline & $\infty$ & $\dot{q}$ & $\ddot{0}$ & $\vdots$ & $\vdots$ & $\vdots$ & $\vdots$ & $\vdots$ & $\vdots$ & $\vdots$ & $\vdots$ & $\vdots$ \\
\hline & - & s & $\infty$ & 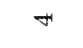 & 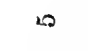 & 0 & 1 & $\infty$ & $\infty$ & $\circ$ & $=$ & $\stackrel{ }{\sim}$ \\
\hline
\end{tabular}


From line 4,

$$
\begin{aligned}
& x+0.513 y+0 \cdot() 87 z=-0.323, \text { and } x=0.3517 . \\
& {[\alpha x]+0.513[\alpha \beta]+0.087[\alpha \gamma]=0.125 \text {, and }[\alpha x]=0.271 \text {. }}
\end{aligned}
$$

Hence the complete solution is:

$$
\begin{array}{rrr}
x=0.3517 & \text { weight } 3.68 . \\
y=-0.0044 & " & 1.86 . \\
z=0.3453 & \text { " } & 4.50 .
\end{array}
$$

Changing back to the original units

$$
\begin{array}{ll}
x=351 \cdot 7 & p_{x}=3 \cdot 68 \\
y=-4 \cdot 4 & p_{y}=1 \cdot 86 . \\
z=345 \cdot 3 & p_{z}=4 \cdot 50 .
\end{array}
$$

The method here used may be extended to any number of unknowns. If we have to solve a series of normal equations involving a large number of unknowns, where the weights of only a few of the unknowns are required, it is advisable to make these unknowns the last in the order of elimination. If the weights of only two unknowns are required, the solution may be carried out as in table $\mathrm{A}$, page 95 , without the addition of extra columns. The coefficient of the last unknown in the final elimination equation will give the weight of that unknown, and the weight of the last unknown but one can be most simply derived by, the use of the expression derived on page 107. If it should be required to find the weights of more than two unknowns, it is generally better to introduce an extra column into the table of solution, for each unknown whose weight is required. The weights are then evaluated as part of the general solution of the normal equations. It should be noted that the $T$ column may be omitted from the table of solution for three unknowns, since the weight of the last unknown is the coefficient of that unknown in the final elimination equation.

50. The order of solution shown in the examples worked out in detail in this chapter can be varied to some extent to suit the individual taste of the computer. When the coefficients in the normal equations are given to three or four significant figures (or more), it will generally be found convenient to solve by the Gauss 
method of substitution, or some variant of that method, particularly if there are more than three unknowns. If there are only three unknowns some computers prefer the determinant method, particularly when the work is carried out by the use of an arithmometer. When the coefficients in the normal equations are small integers the determinant method may be used; or it may happen that the equations may be more conveniently solved by the methods of elementary algebra, as in Examples 1 and 6 at the end of Chapter V.

The following example illustrates a method of solving the normal equations, and of finding the weights of the unknowns, by simple algebra. A set of four normal equations is given, and the values of the unknowns and their weights are required. The constant terms in the normal equations are written in literal form.

$$
\begin{aligned}
& 3 x+2 y+2 z+2 w=[a l] . \\
& 2 x+3 y+2 z+2 w=[b l] . \\
& 2 x+2 y+3 z+2 w=[c l] . \\
& 2 x+2 y+2 z+3 w=[d l] .
\end{aligned}
$$

Adding all four equations, we find

$$
9(x+y+z+w)=[a l]+[b l]+[c l]+[d l] .
$$

Adding together equations (1) and (2),

or

$$
\begin{aligned}
& 4(x+y+z+w)+x+y=[a l]+[b l], \\
& x+y=\frac{5}{9}[a l]+\frac{5}{9}[b l]-\frac{4}{9}[c l]-\frac{4}{9}[d l] .
\end{aligned}
$$

Subtracting (2) from (1),

From (5) and (6)

$$
x-y=[a l]-[b l]
$$

$$
\begin{aligned}
& x=\frac{7[a l]-2[b l]-2[c l]-2[d l]}{9}, \\
& y=\frac{-2[a l]+7[b l]-2[c l]-2[d l]}{9},
\end{aligned}
$$

with similar forms for $z$ and $w$.

The values of the unknowns can be deduced by putting into these expressions the arithmetical values of $[a l]$, etc. Clearly the weights of all the unknowns are the same, being equal to $\frac{7}{9}$. 
Example 1. The normal equations solved ahove (1) 110) were derived from the following set of observational equations :

$\begin{array}{lllll}1.000 x & -0.061 y & +0.907 z & =-18 & +20 \\ 1.000 & -0.051 & +0.900 & =-41 & -1 \\ 1.000 & +0.291 & -0.634 & =-538 & +34 \\ 1.000 & +0.299 & -0.668 & =-589 & -5 \\ 1.000 & +0.315 & -0.736 & =-650 & -49 \\ 1.000 & +0.999 & -0.817 & =-139 & -66 \\ 1.000 & +1.026 & +0.733 & =-58 & +44 \\ 1.000 & +1.288 & -0.621 & =-549 & +23\end{array}$

Verify the residuals given in the last column, and show that the P.E. of an observational equation is $\pm 31 \%$. Hence show

$$
\begin{aligned}
& r_{x}= \pm 16 \cdot 5, \\
& r_{y}= \pm 23 \cdot 0, \\
& r_{z}= \pm 14 \cdot 8 .
\end{aligned}
$$

Eirample. 2. Find the weight of $y$ in the table above, without using the columns $R, S, T$. (Vide page 107.)

Would it be possible to find the weight of $x$ in the same way? If not, what fresh auxiliary products would have to be ealculated, in order to yield $p_{x}$ without using column $R$ ?

\section{Alternative Proof of the Rule for finding Weights} of the Unknowns.

If $r$ be the P.E. of an observational equation of unit weight, $p_{x}, p_{y}, p_{z}$, etc., the weights of the unknowns, and $r_{x}, r_{y}, r_{z}$, etc., the probable errors of the unknowns, then

$$
p_{x} r_{x}{ }^{2}=r^{2}=p_{y} r_{y}{ }^{2}=p_{z} r_{z}{ }^{2}=\text { etc. }
$$

It has already been shown in $\$ 45$ that we may write

$$
\begin{aligned}
& x=\alpha_{1} l_{1}+\alpha_{2} l_{2}+\ldots=[\alpha l], \\
& y=\beta_{1} l_{1}+\beta_{2} l_{2}+\ldots=[\beta l], \\
& z=\gamma_{1} l_{1}+\gamma_{2} l_{2}+\ldots=[\gamma l],
\end{aligned}
$$

where the $\alpha$ 's, $\beta$ 's, and $\gamma$ 's are constants whose values depend entirely on the values of the coefficients $a_{1}, b_{1}, c_{1}, a_{2}, b_{2}, c_{2}$, etc., of the observational equations.

Since $r$ is the P.E. of each observed quantity $l_{1}, l_{2}$, etc.,

$$
\begin{gathered}
r_{x}^{2}=\left(\alpha_{1}^{2}+\alpha_{2}^{2}+\ldots\right) r^{2}=[\alpha \alpha] r^{2} . \\
\therefore p_{x}=\frac{1}{[\alpha \alpha]} .
\end{gathered}
$$

And similarly $\quad p_{y}=\frac{1}{[\beta \beta]}, \quad p_{z}=\frac{1}{[\gamma \gamma]}$.

B. 0 . 
We now have to find the values of $[\alpha \alpha],[\beta \beta],[\gamma \gamma]$, etc.

In the subsequent work we shall limit our discussion to the case of three unknowns, but the results will all hold for any number of unknowns.

Substituting $x=[\alpha l], y=[\beta l], z=[\gamma l]$, in the normal equations, we find

$$
\begin{aligned}
& {[a a][\alpha l]+[a b][\beta l]+[a c][\gamma l]-[a l]=0,} \\
& {[a b][\alpha l]+[b b][\beta l]+[b c][\gamma l]-[b l]=0,} \\
& {[a c][\alpha l]+[b c][\beta l]+[c c][\gamma l]-[c l]=0 .}
\end{aligned}
$$

These relations must be identically true for all values of the $l$ 's. It follows that the coefficient of each separate $l$ in each of these equations is zero. Collecting these coefficients and equating them to zero, we obtain three sets of $n$ equations each :

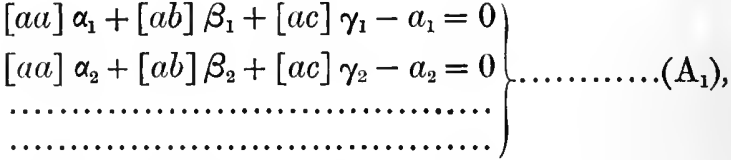

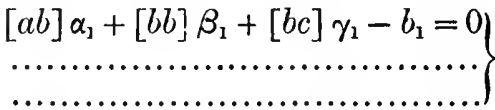

$$
\begin{aligned}
& {[a c] \alpha_{1}+[b c] \beta_{1}+[c c] \gamma_{1}-c_{1}=0} \\
& \}
\end{aligned}
$$

Multiplying the equations in each set by $a_{1}, a_{2}$, etc., and adding, we obtain the three equations :

$$
\begin{aligned}
& {[a a][a \alpha]+[a b][a \beta]+[a c][a \gamma]-[a a]=0,} \\
& {[a b][a \alpha]+[b b][a \beta]+[b c][a \gamma]-[a b]=0,} \\
& {[a c][a x]+[b c][a \beta]+[c c][a \gamma]-[a c]=0 .}
\end{aligned}
$$

This is a set of three equations homogeneous in three unknowns, $[a \alpha]-1,[a \beta]$, and $[a \gamma]$, having no relation between the coefficients; i.e. they are three independent equations. It follows that each variable must vanish.

Similarly it might be shown that

$$
[a \alpha]=1, \quad[a \beta]=0, \quad[a \gamma]=0
$$

$$
\begin{array}{lll}
{[b \alpha]=0,} & {[b \beta]=1,} & {[b \gamma]=0} \\
{[c \alpha]=0,} & {[c \beta]=0,} & {[c \gamma]=1}
\end{array}
$$


Now multiply equations $\left(\Lambda_{1}\right)$ by $\alpha_{1}, \alpha_{2}$, etc., and add

$$
[a a][\alpha \alpha]+[a b][\alpha \beta]+[a c][\alpha \gamma]=[a \alpha]=1
$$

Similarly from $\left(A_{2}\right)$ and $\left(A_{3}\right)$,

$$
\begin{aligned}
& {[a b][\alpha a]+[b b][\alpha \beta]+[b c][\alpha \gamma]=[b \alpha]=0} \\
& {[a c][\alpha \alpha]+[b c][\alpha \beta]+[c c][\alpha \gamma]=[c \alpha]=0}
\end{aligned}
$$

The equations $\left(\mathrm{C}_{1}\right)$ involve three unknowns $[\alpha \alpha],[\alpha \beta],[\alpha \gamma]$. It should be noted that they are the same as the original set of normal equations, with $[\alpha \alpha],[\alpha \beta],[a \gamma]$ substituted for $x, y, z$, and with $[a l]=1,[b l]=[c l]=0$. Hence we may derive immediately the rule for finding $[\alpha \alpha]$ already given in $\S 45$ above.

$[\alpha \alpha]$ is the value of $x$ derived from the normal equations when

$$
[a l]=1, \quad[b l]=0, \quad[c l]=0 .
$$

If equations (A) were multiplied by $\beta_{1}, \beta_{2}$, etc., and then by $\gamma_{1}, \gamma_{2}$, etc., we should derive two sets of equations $\left(\mathrm{C}_{2}\right),\left(\mathrm{C}_{3}\right)$, similar to $\left(\mathrm{C}_{1}\right)$, from which we should derive the values of $[\beta \beta]$ and $[\gamma \gamma]$.

$[\beta \beta]$ is the value of $y$ derived from the normal equations when

$$
[a l]=0, \quad[b l]=1, \quad[c l]=0 ;
$$

and $[\gamma \gamma]$ is the value of $z$ derived from the normal equations when

$$
[a l]=0, \quad[b l]=0, \quad[c l]=1 .
$$

\section{Alternative Proof of the Formula}

$$
r=0.6745 \sqrt{\frac{[p \text { vv] }}{n-m}} \text {. }
$$

For the sake of simplicity in writing we shall consider the case of three unknowns; or $m=3$.

The observational equations, $n$ in number, are

$$
\begin{gathered}
a_{1} x+b_{1} y+c_{1} z-l_{1}=v_{1}=a_{1}[\alpha l]+b_{1}[\beta l]+c_{1}[\gamma l]-l_{1}, \\
a_{2} x+b_{2} y+c_{2} z-l_{2}=v_{2}=a_{2}[a l]+b_{2}[\beta l]+c_{2}[\gamma l]-l_{2}, \\
\text { etc. } \\
v_{1}=l_{1}\left(a_{1} \alpha_{1}+b_{1} \beta_{1}+c_{1} \gamma_{1}-1\right)+l_{2}\left(a_{1} \alpha_{2}+b_{1} \beta_{2}+c_{1} \gamma_{2}\right)+\text { etc., } \\
v_{2}=l_{1}\left(a_{2} \alpha_{1}+b_{2} \beta_{1}+c_{2} \gamma_{1}\right)+l_{2}\left(a_{2} \alpha_{3}+b_{2} \beta_{2}+c_{2} \gamma_{2}-1\right)+\text { etc., } \\
\text { etc. }
\end{gathered}
$$

If the observations were perfect, the values of $l_{1}, l_{2}$, etc., would be absolutely accurate, and the residuals $v_{1}$, $v_{2}$, etc., would all be zero. Let $d l_{1}, d l_{2}$, etc., be the errors in $l_{1}, l_{2}$, etc. Then we may write

$$
v_{1}=\left(a_{1} \alpha_{1}+b_{1} \beta_{1}+c_{1} \gamma_{1}-1\right) d l_{1}+\left(a_{1} \alpha_{2}+b_{1} \beta_{2}+c_{1} \gamma_{2}\right) d l_{2}+\text { etc. }
$$


The quantities $l_{1}, l_{2}$, etc., are all determined independently, and so the errors $d l_{1}$, $d l_{2}$, etc., are all independent, though their mean square errors are all equal. Let this M.S.E. be $\mu$.

Then the mean value of $v_{1}^{2}$ is given by

$$
\begin{aligned}
v_{1}^{2} & =\mu^{2}\left\{\sum_{r=1}^{r=n}\left(a_{1} \alpha_{r}+b_{1} \beta_{r}+c_{1} \gamma_{r}\right)^{2}-2\left(a_{1} \alpha_{1}+b_{1} \beta_{1}+c_{1} \gamma_{1}\right)+1\right\} \\
= & \mu^{2}\left\{a_{1}^{2}[\alpha \alpha]+b_{1}^{2}[\beta \beta]+c_{1}^{2}[\gamma \gamma]+2 a_{1} b_{1}[\alpha \beta]+2 a_{1} c_{1}[\alpha \gamma]\right. \\
& \left.+2 b_{1} c_{1}[\beta \gamma]-2\left(a_{1} \alpha_{1}+b_{1} \beta_{1}+c_{1} \gamma_{1}\right)+1\right\} .
\end{aligned}
$$

We may repeat this process for each residual. Then we obtain the equation

$$
\begin{aligned}
& {[v v]=\mu^{2}\{[a a][\alpha \alpha]+[b b][\beta \beta]+[c c][\gamma \gamma]+2[a b][\alpha \beta]} \\
&+2[a c][\alpha \gamma]+2[b c][\beta \gamma]-2[a \alpha+b \beta+c \gamma]+n\} \\
&=\mu^{2}\{[a c][\alpha \alpha]+[a b][\alpha \beta]+[a c][\alpha \gamma] \\
&+[a b][\alpha \beta]+[b b][\beta \beta]+[b c][\beta \gamma] \\
&+[a c][\alpha \gamma]+[b c][\beta \gamma]+[c c][\gamma \gamma] \\
&-2[a \alpha]-2[b \beta]-2[c \gamma]+n\},
\end{aligned}
$$

and referring back to equations $(B)$ and $(C)$, above, we find

$$
[v v]=\mu^{2}\{n+3-6\}=\mu^{2}(n-3) .
$$

Whence we obtain the usual equation

$$
r=0.6745 \mu=0.6745 \sqrt{\frac{[v v]}{n-3}} .
$$

For the case of $m$ unknowns, the equation derived above would be and then

$$
[v v]=\mu^{2}\{n-2 m+n\}=\mu^{2}(n-m),
$$

$$
r=0.6745 \sqrt{\frac{[v v]}{n-m}} \text { or } 0.6745 \sqrt{\frac{[p v v]}{n-m}} \text {. }
$$

The working is easily modified to apply to the case where the observational equations have unequal weights.

\section{Probable Error of a Function of the Unknowns.}

Let the given function be $f(X, Y, Z)$. As in previous work, the function $f$ may be reduced to a linear form,

or

$$
\begin{gathered}
f(X, Y, Z)=f\left(X_{0} Y_{0} Z_{0}\right)+\frac{\partial f}{\partial X_{0}} x+\frac{\partial f}{\partial \bar{Y}_{0}} y+\frac{\partial f}{\partial Z_{0}} z, \\
d f=\frac{\partial f}{\partial X_{0}} x+\frac{\partial f}{\partial Y_{0}} y+\frac{\partial f}{\partial Z_{0}} z,
\end{gathered}
$$

where

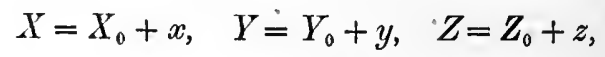


$X_{0}, Y_{0}$, and $Z_{0}$ being approximate values of the unknowns. Since the errors of $x, y, z$ are not independent, we cannot apply to the last equation the reasoning of $\$ \mathbf{2 2}$. With the usual notation,

and

$$
\begin{gathered}
x=[\alpha l], \quad y=[\beta l], \quad z=[\gamma l], \\
d f=\left(\frac{\partial f}{\partial X_{0}}\right)[\alpha l]+\frac{\partial f}{\partial Y_{0}}[\beta l]+\frac{\partial f}{\partial Z_{0}}[\gamma l] \\
=\sum_{r=1}^{r=n}\left(\alpha_{r} \frac{\partial f}{\partial X_{0}}+\beta_{r} \frac{\partial f}{\partial Y_{0}}+\gamma_{r} \frac{\partial f}{\partial Z_{0}}\right) l_{r} .
\end{gathered}
$$

Now the P.E.'s of all the l's are the same, being the P.E. $r$ of an observational equation of unit weight. Hence if $r_{F}$ be the P.E. of the given function $f$, we may write

$$
\begin{aligned}
r_{F^{2}}= & r^{2} \Sigma\left(\alpha_{r} \frac{\partial f}{\partial X_{0}}+\beta_{r} \frac{\partial f}{\partial Y_{0}}+\gamma_{r} \frac{\partial f}{\partial Z_{0}}\right)^{2} \\
= & r^{2}\left\{[\alpha \alpha]\left(\frac{\partial f}{\partial X_{0}}\right)^{2}+[\beta \beta]\left(\frac{\partial f}{\partial Y_{0}}\right)^{2}+[\gamma \gamma]\left(\frac{\partial f}{\partial Z_{0}}\right)^{2}\right. \\
& \left.+2[\alpha \beta] \frac{\partial f}{\partial X_{0}} \frac{\partial f}{\partial Y_{0}}+2[\beta \gamma] \frac{\partial f}{\partial Y_{0}} \frac{\partial f}{\partial Z_{0}}+2[\alpha \gamma] \frac{\partial f}{\partial X_{0}} \frac{\partial f}{\partial Z_{0}}\right\} .
\end{aligned}
$$

It should be noted that in general it is not correct to write

$$
r_{F}{ }^{2}=\left(\frac{\partial f}{\partial X_{0}}\right)^{2} r_{x}{ }^{2}+\left(\frac{\partial f}{\partial Y_{0}}\right)^{2} r_{y}{ }^{2}+\left(\frac{\partial f}{\partial Z_{0}}\right) r_{z}{ }^{2}
$$

The R.H.S. of the last equation is equivalent to the first three terms on the R.H.S. of the previous equations. In general the other terms do not vanish.

A complete solution of a set of normal equations includes the determination of the weights of the unknowns; i.e. it requires the evaluation of $[\alpha \alpha],[\beta \beta],[\gamma \gamma]$. If the method of the table on page 110 above is followed, the values of $[\alpha \beta],[\beta \gamma],[\alpha \gamma]$ are also derived in the course of the complete solution. It is thus practicable to evaluate $r_{F}$ from the equation derived here.

\section{Normal Place Method in Formation of Observa-} tional Equations.

It sometimes happens that a series of observations are such that referred to one of the variable circumstances, say the time, they cluster in groups round certain values of that variable, with well-marked gaps between successive groups. In such a case there 
is generally no appreciable loss of accuracy in the resulting solution if the mean of each group is taken, and associated with the mean value of the variable (time). Such a mean value is called a normal place. The use of the normal place instead of the individual observations reduces the number of observational equations, and produces a considerable saving in time and labour in the formation of the normal equations. For example, if observations of a planet are made at intervals of a fortnight, it is perfectly legitimate to take the mean of one night's observations to form a normal place for working ont the elements of the orbit of the planet; provided that the orbit of the planet is not affected by any disturbing cause of short period.

\section{Testing the Results of the Least Square Solution} for Unusual Errors, and for Systematic or Constant Errors.

When the normal equations have been solved, yielding the values of the unknowns, the next step in the work is to form the residuals by substituting the derived values of the unknowns in the observational equations. The sum of the squares of the residuals will be required in evaluating the probable errors, and even if it should be convenient to evaluate this sum by any other method, the value of $[v v]$ derived directly from the observational equations affords a useful check upon the work of solution. Again it may happen that some of the observations are affected by sources of error not present in the other observations. The effect of such unusual errors would be to yield unusually high values to the corresponding residuals. If some of the residuals should be large in comparison with the probable errors, it may be advisable to reject the observations which yield the high residuals. The problem of the rejection of observations will be considered more fully in Chapter VIII. Meanwhile we note in passing that the evaluation of the residuals is an essential stage in the process of testing the value of the different observations. Further it should be noted that if some of the observations are rejected, it is necessary to repeat the work of solution, as the coefficients in the normal equations are all slightly modified. The values of the unknowns obtained in the first solution may be taken as approximate values, for which the new normal equations will yield corrections. 
All the methods of solution hitherto considered are based on the assumption that the observations are affected only by accidental errors, all constant and systematic errors having been removed. The distinction between constant, systematic, and accidental crrors has already been discussed in Chapter I above. It was suggested that constant and systematic errors shoull be eliminated either by changes in the method of solution, or by empirical corrections deduced from special observations designed to determine the exact law of such errors. In practice, however, it is seldom possible to eliminate all the constant and systematic errors, simply because we can never know the nature of all the errors to which an observation is subject. Errors of theory give rise to incorrect coefficients in the observational equations, and these in turn enter into the coefficients of the normal equations, and so affect the values of the unknowns. And when the values of the unknowns are substituted in the normal equations, the errors of theory affect the values of the residuals, and appear in effect as systematic errors.

It is even more important to consider the systematic errors than the accidental errors, since the latter are eliminated by mere repetition, or by the mere increase in the number of observations. The methods of observation should be so arranged as to avoid systematic errors, as far as possible, or to provide corrections for such systematic errors as are not eliminated by the methods of observation. Finally, the residuals may be made to yield information concerning the presence of systematic errors. A number of methods suggest themselves.

1. A comparison of the actual error curve with the theoretical error curve. Thus in $\$ 24$, Examples 4 and 5, the curves of actual error show a well-marked deviation from the form of the normal error curve, so suggesting the presence of a systematic error. In Example 4 it was possible to suggest a plausible explanation of this error.

The clustering of points in the diagram representing the observations would also point to the presence of a systematic error.

2. If the residuals show a tendency to have a certain sign when a certain set of conditions exist, and the opposite sign when these conditions are absent, or when other conditions exist, the result may be ascribed to systematic error. 
3. When the observations extend over a long period of time, the residuals should be arranged in order of date of observation. If the residuals so arranged follow a systematic law of variation, a systematic error may be expected. For example, an observer knowing nothing of the aberration of light, would find differences between the observed places of stars in different months. But if the observations of one star extended over a number of years, and the errors were arranged in order of date of observation, it would be found that the error for any one star was the same at the same time of the year. The systematic error could then be made an object of prediction, and would cease to be an error.

Again if both the residuals and certain of the conditions of the observations, say the temperature, be arranged in order of date of observation, any correspondence between the variations of the residuals and the conditions considered would indicate a systematic error due to those conditions.

4. A comparison of the results of solution with those of an independent set of observations made under different conditions, or by different methods, may help to determine the presence of constant or systematic errors.

\section{MISCELLANEOUS EXAMPLES.}

1. The Hartmann-Cornu formula for the reduction of prismatic spectrograms*.

The usual method of reducing spectrograms, i.e. of measuring the wavelengths of lines in the spectrum by the use of lines of known wave-length, is that due to Hartmann. If $n$ be the measured scale-reading of a line of wave-length $\lambda, n$ and $\lambda$ are connected by the formula

$$
n-n_{0}=\frac{c}{\left(\lambda-\lambda_{0}\right)^{\alpha}}
$$

where $n_{0}, \lambda_{0}$, and $c$ are constants for each plate, and $a$ is constant for all plates taken with the same instrument. The value of $a$ being known for the particular instrument used, $n_{0}, \lambda_{0}$, and $c$ can be approximately determined by measuring the positions of three lines of known wave-length. The values of these constants may then be improved by measuring a number of lines, say 12, and giving the constants such values as will afford the best fit to the 12 lines.

* Vide Monthly Notices, R.A.S., Vol. Lxxi, p. 663, Stratton. 
Corresponding to a line of wave-length $\lambda_{r}$ the scale-reading should be

$$
n_{r}=n_{0}+\frac{c}{\left(\lambda_{r}-\lambda_{0}\right)^{2}}
$$

If this equation is not satisfied, let

$$
\delta n_{r}=n_{r}-\left(n_{\theta}+\frac{c}{\left(\lambda_{r}-\lambda_{\theta}\right)^{\alpha}}\right)
$$

We have to find corrections to the constants $n_{0}, \lambda_{0}$, and $c$, so that equation (2) shall be satisfied.

The equations to be satisfied for the 12 standard lines are

$$
\begin{aligned}
n_{r} & =\left(n_{0}+\partial n_{0}\right)+\frac{c+\partial c}{\left\{\lambda_{r}-\left(\lambda_{0}+\partial \lambda_{0}\right)\right\}^{\alpha}} \quad(r=1,2, \ldots, 12) \\
& =n_{0}+\partial n_{0}+\frac{c}{\left(\lambda_{r}-\lambda_{0}\right)^{\alpha}}+\frac{\partial c}{\left(\lambda_{r}-\lambda_{0}\right)^{\alpha}}+\frac{a c \partial \lambda_{0}}{\left(\lambda_{r}-\lambda_{0}\right)^{\alpha+1}} .
\end{aligned}
$$

Making use of equation (3) we may write these equations as

$$
\partial n_{r}=\partial n_{0}+\frac{\partial c}{\left(\lambda_{r}-\lambda_{0}\right)^{x}}+\frac{a c \partial \lambda_{0}}{\left(\lambda_{r}-\lambda_{0}\right)^{\alpha+1}} \quad(r=1,2, \ldots, 12) .
$$

These form a set of 12 observational equations which may be solved by the methods of least squares. The normal equations are

$$
\begin{aligned}
& 12 \partial n_{0}+\sum_{1}^{12} \frac{1}{\left(\lambda_{r}-\lambda_{0}\right)^{\alpha}} \partial c+\sum_{1}^{12} \frac{a c}{\left(\lambda_{r}-\lambda_{0}\right)^{\alpha+1}} \partial \lambda_{0}=\sum_{1}^{12} \partial n_{r} \\
&+\sum_{1}^{12} \frac{1}{\left(\lambda_{r}-\lambda_{0}\right)^{2 \alpha}} \partial c+\sum_{1}^{12} \frac{a c}{\left(\lambda_{r}-\lambda_{0}\right)^{2 a+1}} \partial \lambda_{0}=\sum_{1}^{12} \frac{\partial n_{r}}{\left(\lambda_{r}-\lambda_{0}\right)^{\alpha}} \\
&+\sum_{1}^{12} \frac{a^{2} c^{2}}{\left(\lambda_{r}-\lambda_{0}\right)^{2 a+2}} \partial \lambda_{0}=\sum_{1}^{12} \frac{a c \partial n_{r}}{\left(\lambda_{r}-\lambda_{0}\right)^{\alpha+1}} .
\end{aligned}
$$

In these equations, all the quantities which occur are known, except $\partial n_{0}, \partial c, \partial \lambda_{0}$, for which these equations have to be solved.

In practice, it is found that when the normal equations are solved, the weights of the corrections which they yield are very small, i.e. the corrections are very badly determined. It is customary to assume $\lambda_{0}$ to remain constant, so that $\partial \lambda_{0}=0$. The normal equations then yield corrections $\partial n_{0}$ and $\partial c$ which are well-determined.

2. Position of the sun's axis*.

In an investigation on the position of the sun's axis, Dyson derived his observational equations in the form

$$
x \cos \theta_{r}+y \sin \theta_{r}+z=l_{r},
$$

where $\theta$ had one of thirteen values, $0^{\circ}, \pm 15^{\circ}, \pm 30^{\circ}, \ldots$, etc., $\pm 90^{\circ}$. Each observed quantity $l_{r}$ was subject to the same probable error $\epsilon$.

* Dyson, Monthly Notices, R.A.S., Vol. Lxxir, p. 564. 
The normal equations, derived in the usual way, are

$x \Sigma \cos ^{2} \theta+y \Sigma \sin \theta \cos \theta+z \Sigma \cos \theta=\Sigma l \cos \theta$, P.E. $\in \sqrt{\Sigma \cos ^{2} \theta}$,

$x \Sigma \sin \theta \cos \theta+y \Sigma \sin ^{2} \theta+z \Sigma \sin \theta=\Sigma l \sin \theta$, P.E. $\in \sqrt{\Sigma \sin ^{2} \theta}$,

$$
x \Sigma \cos \theta+y \Sigma \sin \theta+z 13=\Sigma l, \quad \text { P.E. } \in \sqrt{13},
$$

or, evaluating the trigonometric coefficients,

$$
\begin{aligned}
6 x+7 \cdot 64 z & =a, \text { P.E. } \in \sqrt{6}, \\
7 y & =\beta, \text { P.E. } \in \sqrt{7}, \\
7 \cdot 64 x+13 z & =\gamma, \text { P.E. } \in \sqrt{13} .
\end{aligned}
$$

Thus $y$ is obtained with a P.E. $\frac{\epsilon}{\sqrt{7}}$, but it is found from the actual solution that $x$ is determined with a P.E. $2 \cdot 1 \epsilon$, so that $x$ is not by any means as clearly determined as $y$.

The chief value of the investigation leading to the above set of normal equations therefore consists in the determination of the quantity $y$, which is given by the least squares solution with a P.E. $\frac{\epsilon}{\sqrt{7}}$ or $378 \epsilon$. It is of considerable importance to consider how closely this value can be obtained without going through the least squares solution.

Writing down the 13 observational equations in order, and subtracting the 13 th from the 1 st, the 12 th from the 2 nd, the 11 th from the 3 rd, etc., we obtain the equations

$$
\begin{aligned}
& y=\frac{1}{2}\left(l_{1}-l_{13}\right), \\
& .966 y=\frac{1}{2}\left(l_{2}-l_{12}\right), \\
& .866 y=\frac{1}{2}\left(l_{3}-l_{11}\right), \\
& \text { etc. }
\end{aligned}
$$

Each of these equations has a P.E. $\frac{\epsilon}{\sqrt{2}}$.

Adding the first two of these equations, we obtain 1.966y with a P.E.

$$
\frac{\epsilon}{\sqrt{2}} \sqrt{2} \text {. }
$$

.Adding the first three, we obtain $2 \cdot 832 y$ with a P.E. $\frac{\epsilon}{\sqrt{2}} \sqrt{3}$; and so on. In this way $y$ is determined

with P.E. $\cdot 707 \epsilon$ using one equation at each end of the series of thirteen,

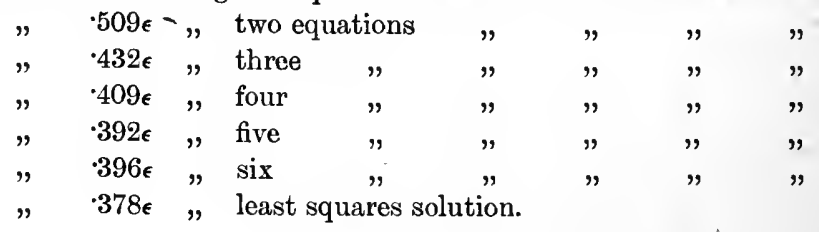


By using five equations at each end of the series we thus obtain a very elose approximation to the accuracy of a least squares solution, while four equations at each end only increase the P.E. from $38 \mathrm{\epsilon}$ to $41 \mathrm{\epsilon}$. The use of four equations at eneh end of the series yields results of a high degree of accuracy, and affords a considerable saving of labour.

The above set of equations illustrates a fact which has wide applications. If in the observational equations two of the unknowns appear with coefficients which are more or less related, e.g. always have the same sign (as in the case of the coefficients of $x$ and $z$ in the above equations), their weights in the solution will be small. If the coefficient of $z$ were always twice that of $x$ we could not determine either separately.

3. Solve the normal equations derived above for $x, y, z$, and find the weights of $x$ and $z$. Verify the statement that the P.E. of $x$ is $2 \cdot 1 \epsilon$.

4. Why is it not legitimate to proceed as follows in Example 2 ?

$$
13(6 x+7 \cdot 64 z)-7 \cdot 64(7 \cdot 64 x+13 z)
$$

has P.E. made up of $13 \epsilon \sqrt{6}$ and $7 \cdot 64 \epsilon \sqrt{13}$.

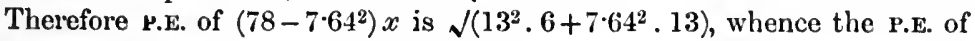
$x$ can be deduced. 


\section{CHAPTER VII}

\section{THE ADJUSTMENT OF CONDITIONED OBSERVATIONS}

56. WHEN the quantities measured, or the unknowns which they involve, are not independent, but are connected $a$ priori by certain relations which must be satisfied by the adjusted values, the methods of Chapters V and VI are not directly applicable.

Let there be $n$ directly observed quantities $M_{1}, M_{2}, \ldots, M_{n}$, of weights $p_{1}, p_{2}, \ldots, p_{n}$, and let the most probable values of the observed quantities be $L_{1}, L_{2}, \ldots, L_{n}$.

Then, if $v_{1}, v_{2}$, etc., be the residuals, we have the relations

$$
\left.\begin{array}{c}
L_{1}-M_{1}=v_{1} \\
L_{2}-M_{2}=v_{2} \\
\text { etc. }
\end{array}\right\}
$$

In Chapters V and VI it was supposed that the quantities $L_{1}, L_{2}$, etc., could be accurately represented as functions of certain unknown quantities $X, Y, Z$, etc., $m$ in number. The same method night be applied to the problem we now have to consider. In general the a priori relations mentioned above are expressible as explicit functions of $L_{1}, L_{2}$, etc., and consequently as explicit functions of $v_{1}, v_{2}$, etc.; and it is more convenient to regard

$$
v_{1}, v_{2}, \ldots, v_{n}
$$

as $n$ unknown quantities, which are connected by a certain number of conditions, or functional relations. Let these relations, $m^{\prime}$ in number, be reduced to linear form (cf. page 76), and written

$$
\left.\begin{array}{l}
h_{1} v_{1}+h_{2} v_{2}+h_{3} v_{3}+\ldots-l_{1}=0 \\
k_{1} v_{1}+k_{2} v_{2}+k_{3} v_{3}+\ldots-l_{2}=0 \\
\ldots \ldots \ldots \ldots \ldots \ldots \ldots \ldots \ldots \ldots \ldots \ldots \ldots \ldots \ldots \ldots \ldots \ldots \ldots \ldots
\end{array}\right\}
$$


where the coefficients $h_{1}, h_{2}, k_{1}, k_{2}, l_{1}, l_{2}$, etc, are all known quantities. The least square theory requires that $[p v v]$ shall be a ininimum subject to the conditions represented by equations (ii). There are two methods of effecting this.

\section{Direct Solution by Substitution.}

By the use of equations (ii) it is possible to express $m^{\prime}$ of the unknown residuals as linear functions of the remaining $n-m^{\prime}$ residuals. Substituting the values thus obtained in $[p v v]$ we obtain an expression involving only $n-m^{\prime}$ independent unknowns. Differentiating this expression with respect to each of these independent unknowns in turn, we obtain $n-m^{\prime}$ linear equations whose solution yields the value of the $n-m^{\prime}$ independent unknowns. The remaining $m^{\prime}$ residuals can be evaluated by the use of the expressions first deduced from the equations (ii). 'The residuals are then all known.

Example 1. The Adjustment of Coplanar Angles.

The following are the observed values of four coplanar angles. What are the most probable values of the angles?

$$
\begin{aligned}
& A O B=80^{\circ} 13^{\prime} 10^{\prime \prime} \text { weight } 3 \text {, } \\
& B O C=83^{\circ} 18^{\prime} 8^{\prime \prime} \quad \text {, 4, } \\
& C O D=72^{\circ} 6^{\prime} 4^{\prime \prime} \quad \text {, 2, } \\
& D O A=124^{\circ} 21^{\prime} 16^{\prime \prime} \quad \text { " } 2 . \\
& \text { Sum }=359^{\circ} 58^{\prime} 38^{\prime \prime} \text {. }
\end{aligned}
$$

Let $x, y, z, w$ be the corrections, measured in seconds, to be added to the measured values of these angles.

- Then

or

$$
\begin{array}{r}
359^{\circ} 58^{\prime} 38^{\prime \prime}+(x+y+z+w)^{\prime \prime}=360^{\circ}, \\
x+y+z+w=82 \ldots \ldots \ldots \ldots . .
\end{array}
$$

This conditional equation must be rigorously satisfied by the adjusted values of the angles.

The observational equations are

$$
\left.\begin{array}{lll}
x=0 & \text { weight } & 3 \\
y=0 & \# & 4 \\
z=0 & \# & 2 \\
w=0 & \# & 2
\end{array}\right\}
$$

Substituting for $w$ from equation (1), we replace the last of equations (2) by

$$
-x-y-z+82=0 \text { weight } 2
$$


The normal equations derived from the first three equations in (2), and equation (3), are

$$
\begin{aligned}
& 5 x+2 y+2 z=164, \\
& 2 x+6 y+2 z=164, \\
& 2 x+2 y+4 z=164 .
\end{aligned}
$$

Subtracting the second equation from the first, and the third from the second, we find

$$
3 x-4 y=0, \quad 4 y-2 z=0 .
$$

Whence we find

or

$$
\begin{array}{lll}
x=3 \frac{28}{9}, & y=\frac{246}{19}, \quad z=\frac{499}{19}, \quad w=\frac{492}{19}, \\
x=17^{\prime \prime}, & y=13^{\prime \prime}, \quad z=26^{\prime \prime}, \quad w=26^{\prime \prime} .
\end{array}
$$

The adjusted values of the angles are therefore

$$
\begin{aligned}
& A O B=80^{\circ} 13^{\prime} 27^{\prime \prime}, \\
& B O C=83^{\circ} 18^{\prime} 21^{\prime \prime}, \\
& C O D=72^{\circ} 6^{\prime} 30^{\prime \prime}, \\
& D O A=124^{\circ} 21^{\prime} 42^{\prime \prime} .
\end{aligned}
$$

Example 2. The three angles of a coplanar triangle are all equally well observed. To find the most probable values of the angles.

Let $A, B, C$ be the three angles, and $\epsilon$ the spherical excess of the triangle.

Then

$$
A+B+C=180^{\circ}+\epsilon \text {. }
$$

Let $M_{1}, M_{2}, M_{3}$ be the observed values of the angles, and let

$$
\begin{aligned}
& A=M_{1}+v_{1}, \\
& B=M_{2}+v_{2}, \\
& C=M_{3}+v_{3} .
\end{aligned}
$$

Then the problem is to find the most probable values of the residuals $v_{1}, v_{2}, v_{3}$,

$$
v_{1}+v_{2}+v_{3}=180^{\circ}+\epsilon-M_{1}-M_{2}-M_{3}=\alpha \text {, say. }
$$

The least square theory requires that

$$
v_{1}^{2}+v_{2}^{2}+v_{3}^{2}
$$

shall be a minimum subject to the condition

Substituting for $v_{3}$, we find that

$$
v_{1}+v_{2}+v_{3}=\alpha \text {. }
$$

must be a minimum.

$$
v_{1}^{2}+v_{2}^{2}+\left(a-v_{1}-v_{2}\right)^{2}
$$

Differentiating with respect to $v_{1}$ and $v_{2}$ in turn, we obtain the equations

Whence we deduce

$$
\begin{aligned}
& 2 v_{1}+v_{2}=\alpha, \\
& v_{1}+2 v_{2}=\alpha .
\end{aligned}
$$

$$
v_{1}=v_{2}=\frac{\alpha}{3}=v_{3}
$$


58. Method of Undetermined Multipliers or Correlates.

The method of direct substitution is only practicable when the relations expressed by equations (ii) are few in number and simple in form. In other cases the minimum value of $[p v v]$ can best be found by the method of "Undetermined Multipliers."

Equations (ii) are multiplied by $-2 A_{1},-2 A_{2}$, etc., and the products are added to $[p v v]$, yielding the expression

$[p v v]-2 A_{1}\left(h_{1} v_{1}+h_{2} v_{2}+\ldots-l_{1}\right)-2 A_{2}\left(k_{1} v_{1}+k_{2} v_{2}+\ldots-l_{2}\right)$ etc. (iii).

We then proceed to find the values of $v$ which will make this expression a minimum. Differentiating with respect to $v_{1}, v_{2}$, etc. in turn, we obtain a system of $n$ equations:

$$
\left.\begin{array}{l}
p_{1} v_{1}=A_{1} h_{1}+A_{2} k_{1}+\ldots \\
p_{2} v_{2}=A_{1} h_{2}+A_{2} k_{2}+\ldots \\
\ldots \ldots \ldots \ldots \ldots \ldots \ldots \ldots \ldots \ldots
\end{array}\right\}
$$

Substituting these values of $v_{1}, v_{2}$, etc. in the equations (ii), we obtain a system of $m^{\prime}$ equations :

$$
\left.\begin{array}{l}
A_{1}\left[\frac{h h}{p}\right]+A_{2}\left[\frac{h k}{p}\right]+\ldots=l_{1} \\
A_{1}\left[\frac{h k}{p}\right]+A_{2}\left[\frac{k k}{p}\right]+\ldots=l_{2}
\end{array}\right\}
$$

where

$$
\begin{aligned}
& {\left[\frac{h h}{p}\right]=\frac{h_{1}^{2}}{p_{1}}+\frac{h_{2}^{2}}{p_{2}}+\ldots \text { etc. }} \\
& {\left[\frac{h k}{p}\right]=\frac{h_{1} k_{1}}{p_{1}}+\frac{h_{2} k_{2}}{p_{2}}+\ldots \text { etc. }}
\end{aligned}
$$

These $m^{\prime}$ equations involve the $m^{\prime}$ correlates $A_{1}, A_{2}$, etc. as unknowns, and the solution yields the values of the correlates. Substituting the values so obtained in equations (iv), we obtain the most probable values of the residuals $v_{1}, v_{2}$, etc. Equations (v) are ealled the normal equations for the correlates $A_{1}, A_{2}$, etc.

Example. Example 1 above might be worked out by this method. We should have to make

a minimum.

$$
3 x^{2}+4 y^{2}+2 z^{2}+2 w^{2}-2 A(x+y+z+w-82)
$$

Differentiating with respect to $x, y, z, w$, in turn, we find

Since

$$
3 x=A=4 y=2 z=2 x \text {. }
$$

$$
x+y+z+w=82
$$

we immediately deduce the same values of $x, y, z, w$ as were given above. 


\section{The Precision of the Unknowns.}

It was shown on page 100 that in a system of $n$ observations involving $m$ independent unknowns, the P.E. of an observational equation of unit weight is

$$
r=0.6745 \sqrt{\frac{[p v v]}{n-m}} .
$$

In the case considered in this chapter there are $n$ unknowns, comnected by $m^{\prime}$ relations. But we can use the $m^{\prime}$ relations (ii) to eliminate $m^{\prime}$ of the unknown quantities, so that we shall have $n$ observed values, involving $n-m^{\prime}$ independent unknowns. We may then use the formula given above for evaluating $r$.

But

$$
\begin{aligned}
& m=n-m^{\prime} \text { or } n-m=m^{\prime}, \\
& \therefore r=0.6745 \sqrt{\frac{[p v v]}{m^{\prime}}} .
\end{aligned}
$$

The P.E. of a residual $v_{s}$ of weight $p_{s}$ is

$$
r_{s}=0.6745 \sqrt{\frac{[p v v]}{m^{\prime} p_{8}}} .
$$

For a full development of the subject of this chapter, and in application to survey work in particular, the reader is referred to

Wright and Hayford, Adjustment of Observations, Chapters v, vi, and vii.

F. R. Helmert, Ausgleichungsrechnung, Chapter iv.

Oscar S. Adams, Application of Theory of Least Squares to the Adjustment of Triangulation. U.S. Coast and Geodetic Survey, Special Publication No. 28.

Jordan, Handbuch der Vermessungskunde, Bd. I. . 


\section{CHAP'TER VIII}

\section{THE REJECTION OF OBSERVATIONS}

60. An observer making a series of observations of any kind has power to reject any observation if he is certain that it is vitiated by some unusual sources of error which do not affect the other observations in the series. To put this in other words, the observer is to a certain extent free to choose the time for making his observations so that the external conditions shall vary as little as possible during the series. $\mathrm{He}$ is supreme in his own department, having the power to retain or reject observations according to his judgment of the extent to which the external causes of error react upon his measurements.

But when the observational material is put into the hands of the computer there arises a new question. Shall the computer be allowed to reject any observation whose residual is much larger than those of the remaining observations? He should clearly be allowed to reject an observation when he is convinced that it is affected by an error from some unusual source, which does not affect the other observations in the series; or if the observation is clearly spoiled by some definite blunder, such as the mis-reading of a scale by five, divisions. It is sometimes possible to correct blunders of this type, and to retain the observation, but the greatest caution is necessary in making such corrections. The real difficulty lies in deciding whether a large residual is due to some unusual source of error, or is due to the chance occurrence of a large number of small accidental errors with the same sign. If the latter alternative be true, the large residual is in accordance with the law of error, and its rejection will decrease rather than increase the accuracy of the final result.

B. 0 . 
A number of criteria, based on more or less rigid analysis, have been put forward by various writers. The best known of these is Peirce's Criterion. The underlying principle is that the doubtful observations should be rejected when the probability of the system of errors obtained by retaining them is less than the product of the probability of the system of errors obtained by rejecting them, multiplied by the probability of making so many, and no more, abnormal observations. As this criterion is not in general use, and is rather tedious in its application, we shall not enter into the full proof. The reader who desires to know more of it is referred to Chauvenet's Theoretical and Practical Astronomy, Vol. II, Appendix, $\S 58$, where Peirce's proof is reproduced almost word for word; and to the proof and tables by Gould in Astronomical Journal, Vol. IV, and U.S. Coust and Geodetic Survey Report, 1854, pp. 131, 132*.

Chauvenet (Vol. II) gives a criterion "for the rejection of one doubtful observation." The probability that an error is less than $t$ is, as we have already seen,

$$
\frac{2}{\sqrt{\pi}} \int_{0}^{\frac{t}{h}} e^{-t^{2}} d t=\Theta(t)=\frac{2}{\sqrt{ } \pi} \int_{0}^{\frac{\rho t}{r}} e^{-t^{2}} d t .
$$

Of a series of $m$ observations, the number whose errors may be expected to be less than $t$ will be $m \Theta(t)$, and the number which will exceed $t$ will be $m(1-\Theta(t))$. If this last quantity be less than $\frac{1}{2}$, then it follows that an error greater than $t$ has a greater probability against it than for it, and may therefore be rejected. The limiting error $t$ which may be rejected is therefore given by

$$
m(1-\Theta(t))=\frac{1}{2} \text { or } \Theta(t)=\frac{2 m-1}{2 m} .
$$

The function $\Theta(t)$ has been tabulated on page 19 .

The application of the criterion by means of the formula given above is extremely simple. Suppose we have a series of 100 observations. Then

$$
\frac{2 m-1}{2 m}=\cdot 995 \text {. }
$$

Referring to page 19 we find this value for $\Theta(t)$ when $\frac{t}{r}=4 \cdot 2$. The

* See also, "Note on Peirce's Criterion," s. A. Saunder, Monthly Notices, R.A.S., Vol. Lxirl, p. 432. 
limiting value of $t$ is 4.2 times the probable error. So that if one of a series of 100 observations has a residual greater than 4.2 times the probable error of the series, Chanvenet's criterion would reject that observation.

Chanvenet's criterion only considers the rejection of one observation, but when one has been rejected, the rule may be applied to consider the rejection of another, and so on. The criterion is simple and easy to apply, but it is probably too sweeping. It does not allow sufficiently for the possible presence of a large number of small accidental errors of the same sign.

Other eriteria have been suggested, but few of them are really useful in practice. The whole question of the possibility of rejecting observations on the ground of theoretical discussion based on residuals only, has given rise to a considerable amount of controversy. Bessel opposed the rejection of any observation unless the observer was satisfied that the external conditions produced some unusual source of error not present in the other observations of the series. Peirce's criterion was at an early date subjected to very severe criticism. Airy* claimed that it was defective in foundation, and illusive in its results. He maintained that, so long as the observer was satisfied that the same sources of error were at work, though in varying degrees, throughout a series of observations, the computer should have no right to reject any observation by a discussion based solely on antecedent probability. An observation should be rejected only when a thorough examination showed that the causes of error normally at work were not sufficient to produce the error in the doubtful observation. Airy also cited a case where the rejection of the observations having large residuals led to poor results. In the preceding century, at a time when the figure of the earth was not well determined, azimuth observations were made at Beachy Head and Dunnose, in connection with the survey of England, and the results obtained were poor. Later on, when the full record of the observations fell into the hands of General. Colby, it was found that only the observations which were in closest agreement had been used in the reduction. When the calculations were repeated, using all the observations, results of a high degree of accuracy were obtained.

* Astronomical Journal, Vol. Iv, p. 137. 
Though many of the arguments of Airy and others against the use of mathematical criteria such as Peirce's have been shown to be based on faulty premises, the fact remains that none of these criteria have ever come into general use.

If the distribution of errors be in strict accordance with the law of error, only a few large residuals will occur. Referring to the table on page 19, we see that the probability of an error greater than $5 r$, where $r$ is the probable error of a single observation, is $\cdot 001$, and the probability of an error greater than $3.5 r$ is 018 . Thus only one observation in 1000 should have an error as great as $5 r$, while one error in 55 should have an error as great as $3.5 r$. These numbers form the theoretical basis of the following rule, which is advocated for general use by Wright and Hayford*.

"Reject each observation for which the residual exceeds five times the probable error of a single observation. Examine carefully each observation for which the residual exceeds 3.5 times the probable error, and reject it if any of the accompanying conditions are such as to produce lack of confidence."

This criterion has the merits of simplicity, ease of application, and a fairly sound theoretical basis. For a moderate number of observations it cannot be said to be too sweeping.

* Adjustment of Observations. 


\section{CHAP'TER IX}

\section{ALTERNATIVES TO THE NORMAL LAW OF ERRORS}

61. WE have already dealt briefly with one or two cases in which the frequency distribution showed a well-marked deviation from the normal form. In economic and biological statistics, in particular, the frequency distributions are liable to show considerable dis-symmetry. It is therefore necessary to consider the methods of representing such frequency distributions by some substitute for the normal error curve of Gauss. The purpose which has to be kept in view is that of replacing the series which represents the observations by a simple formula involving a few constants only.

Before proceeding to the development of possible formulae which shall represent various types of frequency distribution, we must define briefly a number of statistical terms. Let the data be arranged in the form of a frequency distribution, and let $f_{x}$ be the frequency of the characteristic $x$. The characteristic is the scale in terms of which the observations are made. In the diagrams of the earlier chapters of this book, it is the variable represented along the horizontal axis (the $x$-axis).

The median is the value of the characteristic which has as many observations on one side of it as on the other.

The mode is the value of the characteristic corresponding to the maximum ordinate of the frequency curve. The position of this ordinate cannot be accurately determined until the form of the frequency curve is known, since it is not necessarily the ordinate corresponding to the biggest number of actual observations. (Compare figure 7 , page 46 .)

The mean is the average value of the characteristic, and is the arithmetic mean of all the observed values. If $f_{x}$ be the 
frequency of a value $x$ of the characteristic, or the frequency of a group centred round $x$, the mean is

or, for the curve,

$$
\begin{gathered}
\frac{\sum f_{x} x}{\sum f_{x}}, \\
\frac{\int f_{x} x d x}{\int f_{x} d x} .
\end{gathered}
$$

The ordinate through the mean is often spoken of as the centroid vertical, since it passes through the centre of gravity of the distribution.

The standard deviation, or S.D., represented by the symbol $\sigma$, measures the closeness with which the measurements are clustered about the mean. Measuring $x$ from the mean, $\sigma$ is given by

$$
\sigma^{2}=\frac{\sum x^{2} f_{x}}{\sum f_{x}} \text { or } \sigma^{2}=\frac{\int x^{2} f_{x} d x}{\int f_{x} d x} \text {. }
$$

The first or second form is to be used according as the calculation is made from the observations or from the curve. Using the notation of Chapter III, we should have

$$
\sigma^{2}=\frac{[v v]}{n} \text { where } n=\Sigma f_{x} .
$$

The mean square error is given by

$$
\mu^{2}=\frac{[v v]}{n-1} .
$$

Thus $\sigma=\mu \sqrt{\frac{n-1}{n}}$, and when $n$ is large, $\sigma$ and $\mu$ may be regarded as identical. For the present we shall call this quantity the S.D. $(\sigma)$, rather than the M. S. E. $(\mu)$, since it is customary to do so in all works on general statistics. It should be noted that $\sigma$ does not depend upon the actual frequencies, but only on their distribution. It measures the scatter of the observations about the mean.

When a curve is symmetrical, the mean, mode, and median coincide. An unsymmetrical curve is often described as a skew curve. The skewness of a curve or of a frequency distribution is measured by the distance between the mean and the mode. It is convenient to define the skewness by the equation

$$
\text { Skewness }=\frac{\text { Mean }- \text { Mode }}{\text { S. D. }},
$$


so that a curve with positive skewness has the mode to the left of the mean in the usual diagram, or in other words, when the rise to the maximum is more rapid than the fall from the maximum. In a skew curve the mode is the value of the characteristic which has the greatest frequency.

The $n$th moment of a frequency distribution about any ordinate is obtained by multiplying each frequency by the $n$th power of its distance from the ordinate in question, and adding together all the products. With our present notation, the $n$th moment about the ordinate $x=a$ is

$$
\Sigma f_{x}(x-a)^{n} \text {. }
$$

It is customary to employ the symbol $\mu_{n}$ to represent the $n$th moment about the mean, and the accented symbol $\mu_{n}{ }^{\prime}$ to denote the $n$th moment about any other ordinate. It is generally more convenient to evaluate moments about some other ordinate than the mean, e.g. an ordinate corresponding to an integral value of the characteristic, and then to deduce from these moments the corresponding moments about the mean. Let the distance between the assumed ordinate and the mean be $a$, and let the distance from any other ordinate to these two ordinates be $x_{r}^{\prime}$ and $x_{r}$ respectively. Then $x_{r}=x_{r}{ }^{\prime}-a$.

$$
\begin{aligned}
\mu_{n} & =\Sigma f_{r}\left(x_{r}{ }^{\prime}-a\right)^{n} \\
& =\Sigma f_{r} x_{r}{ }^{\prime n}-n a \Sigma f_{r} x_{r}{ }^{n-1}+\text { etc. } \\
& =\mu_{n}{ }^{\prime}-n a \mu_{n-1}^{\prime}+\frac{n \cdot(n-1)}{1.2} a^{2} \mu_{n-2}^{\prime}-\text { etc. }
\end{aligned}
$$

Or, we may write

$$
\begin{aligned}
\mu_{n}{ }^{\prime} & =\Sigma f_{n}\left(x_{r}^{\prime}\right)^{n}=\Sigma f_{r}\left(x_{r}+a\right)^{n} \\
& =\mu_{n}+n a \mu_{n-1}+\frac{n \cdot n-1}{1.2} a^{2} \mu_{n-2}+\ldots
\end{aligned}
$$

Whence it follows that

$$
\mu_{n}=\mu_{n}{ }^{\prime}-n a \mu_{n-1}-\frac{n \cdot n-1}{1.2} a^{2} \mu_{n-2}-\text { etc. }
$$

Either of equations (1) and (2) may be used to evaluate the moments in turn, the two equations of necessity leading to identical results. 
62. Most of the methods of fitting curves other than the normal error curve, to series of observations, are based on the use of moments. If the functional form contains $n$ constants, these are deduced by making the moments deduced from the observations agree with the moments deduced from the curve, up to the $n$th moment. This process yields $n$ equations which suffice to determine the $n$ constants. The following simple example may help to illustrate the utility of the method.

It is required to fit a curve of the form

$$
y=a+b x+c x^{2}
$$

to the frequency distribution

$$
\begin{array}{rr}
x=1 & y=10 \\
2 & 12 \\
3 & 18 \\
4 & 36
\end{array}
$$

If only the first three terms corresponding to $x=1,2$, and 3 , were given, then by substituting the values of $x$ and $y$ in the assumed equation we could solve the resulting three equations, and so obtain $a, b$, and $c$. The results so obtained are

$$
a=12, \quad b=-4, \quad c=2 .
$$

Thus the curve which accurately fits the first three terms is

$$
y=12-4 x+2 x^{2}
$$

But when we substitute $x=4$ in this equation we find $y=28$. Thus the curve does not fit the fourth term $x=4, y=36$. If any three out of the four points given above were selected, a curve could be made to pass through these three points, but it would not pass through the fourth point. It is therefore necessary to adopt some method of calculating the constants $a, b, c$, so that all four points will be taken into account. The result will be to yield a curve which will pass very near to all four points, but which need not of necessity pass through any one of them. This could easily be done by the method of least squares. Another simple method is to adopt values of the constants $a, b, c$, such that the first three moments of the actual distribution about the origin shall be equal to the first three moments yielded by the curve. 
Taking the zero moment, and the first and second moments, we obtain three equations:

$$
\begin{aligned}
\left(a^{2}+b+c\right)+\quad & \left(a+2 b+2^{2} c\right)+\left(a+3 b+3^{2} c\right)+\left(a+4 b+4^{2} c\right) \\
& =10+12+18+36,
\end{aligned}
$$

$1(a+b+c)+2\left(a+2 b+2^{2} c\right)+3\left(a+3 b+3^{2} c\right)+4\left(a+4 b+4^{2} c\right)$

$$
=10.1+12.2+18.3+36.4 \text {, }
$$

$1^{2}(a+b+c)+2^{2}\left(a+2 b+2^{2} c\right)+3^{2}\left(a+3 b+3^{2} c\right)+4^{2}\left(a+4 b+4^{2} c\right)$

$$
=101^{2}+12.2^{2}+18.3^{2}+36.4^{2} \text {, }
$$

or,

$$
\begin{aligned}
4 a+10 b+30 c & =76, \\
10 a+30 b+100 c & =232, \\
30 a+100 b+354 c & =796 .
\end{aligned}
$$

The solution of these equations yields

$$
a=18, \quad b=-11 \cdot 6, \quad c=4 .
$$

The resulting curve is

$$
y=18-11 \cdot 6 x+4 x^{2}
$$

\begin{tabular}{|c|c|c|}
\hline$x$ & Calculated $y$ & Observed $y$ \\
\hline 1 & $10 \cdot 4$ & 10 \\
2 & $10 \cdot 2$ & 12 \\
3 & $19 \cdot 2$ & 18 \\
4 & $35 \cdot 6$ & 36 \\
\hline
\end{tabular}

The table shows that the adopted curve does not fit any of the observations accurately, but yields values of $y$ which are sometimes greater, sometimes less, than the observed values.

This simple example may help to illustrate the general nature of statistical problems, where it is necessary to replace the irregularities of observations by a smooth curve. 


\section{Pearson's Curves.}

The general theory of curve-fitting has been worked out in great detail by Prof. Karl Pearson*. Starting from certain properties which may be considered essential in good observations, Pearson has derived a series of formulae for possible curves of presumptive errors.

(1) The expression must replace the rough material of observation by a smooth continuous curve; i.e. it must graduate the observations.

(2) The expression must not involve too many constants, and those present must be calculable from the material of observation.

(3) There must be a systematic method of approaching frequency distributions.

(4) If the material is homogeneous, the ordinate of the curve will start from zero, increase to a maximum, and fall again, possibly at a different rate, to zero.

(5) The frequency curve will generally have contact with the axis of $x$ at the ends of the range.

Of these, (1), (2) and (3) call for no remark, while (4) and (5) are properties which are generally associated with the frequency distributions obtained in actual practice. The conditions are in general satisfied by the curve whose equation is

$$
\frac{1}{y} \frac{d y}{d x}=\frac{x+a}{f(x)} \text {. }
$$

For, $\frac{d y}{d x}=0$ when $y=0$, so that the curve has contact at the axis, and $\frac{d y}{d x}=0$ when $x=-a$, corresponding to a maximum of the curve.

Expanding by Maclaurin's Theorem, we may write

$$
f(x)=b_{0}+b_{1} x+b_{2} x^{2}+\ldots \text { etc. }
$$

Pearson has considered in detail the case where $f(x)$ is limited to the first three terms in the expansion. Four constants are then involved, requiring the evaluation of four moments. In general there is little advantage in considering formulae which require

\footnotetext{
* Phil. Trans. R.S. 186 А, p. 343 ; Biometrika, v, p. 172.
} 
the evaluation of higher moments, sinee the higher moments are very sensitive to errors in the frequency distribution. We shall therefore start from the differential equation

$$
\frac{1}{y} \frac{d y}{d x}=\frac{x+a}{b_{0}+b_{1} x+b_{2} x^{2}}
$$

Pearson's method eonsists essentially in making the moments calculated from the curve equal to the moments derived from the observations. The differential equation may be written

$$
\left(b_{0}+b_{1} x+b_{2} x^{2}\right) \frac{d y}{d x}=y(x+a) .
$$

Multiplying each side by $x^{n}$, and integrating, we find

$$
\int x^{n}\left(b_{0}+b_{1} x+b_{2} x^{2}\right) \frac{d y}{d x} d x=\int y(x+a) x^{n} d x .
$$

Integrating the L. H.s. by parts, treating $\frac{d y}{d x}$ as one part, we find

$$
\begin{gathered}
x^{n}\left(b_{0}+b_{1} x+b_{2} x^{2}\right) y-\int\left\{n b_{0} x^{n-1}+(n+1) b_{1} x^{n}+(n+2) b_{2} x^{n+1}\right\} y d x \\
=\int y x^{n+1} d x+a \int y x^{n} d x .
\end{gathered}
$$

But sinee $y$ becomes zero at each end of the range, we find, in terms of our previous notation,

$$
\begin{gathered}
-n b_{0} \mu_{n-1}^{\prime}-(n+1) b_{1} \mu_{n}{ }^{\prime}-(n+2) b_{2} \mu_{n+1}^{\prime} \\
=\mu_{n+1}^{\prime}+a \mu_{n}{ }^{\prime},
\end{gathered}
$$

or $\quad a \mu_{n}^{\prime}+n b_{0} \mu_{n-1}^{\prime}+(n+1) b_{1} \mu_{n}^{\prime}+(n+2) b_{2} \mu_{n+1}^{\prime}=-\mu_{n+1}^{\prime}$.

Putting $n=0,1,2,3$, in turn, in this equation, we might obtain four equations to determine the four constants $a, b_{0}, b_{1}, b_{2}$. These equations were derived independently of any assumption as to the position of the origin. If the origin be at the mean, the aecents may be omitted, and $\mu_{1}$ may be equated to zero. 'The four equations then become

$$
\left.\begin{array}{ll}
n=0 & a+b_{1}=0 \\
n=1 & b_{0}+3 b_{2} \mu_{2}=-\mu_{2} \\
n=2 & a \mu_{2}+3 b_{1} \mu_{2}+4 b_{2} \mu_{3}=-\mu_{3} \\
n=3 & a \mu_{3}+3 b_{0} \mu_{2}+4 b_{1} \mu_{3}+5 b_{2} \mu_{4}=-\mu_{\downarrow}
\end{array}\right\}
$$


Sheppard's Corrections. The $\mu$ 's in these equations represent moments calculated from the curve. In practice, however, the moments are calculated from grouped frequencies, where all values of the characteristic within a certain interval are regarded as equal to the value at the centre of that interval. The moments so calculated are not precisely the same as the moments calculated from the curve. If $\nu_{1}, \nu_{2}$, etc. be the moments calculated from grouped frequencies, Sheppard* has shown that when there is high contact at the axis, certain corrections must be applied to the moments. The relations between the various moments are shown by the following equations :

$$
\left.\begin{array}{l}
\mu_{1}=\nu_{1} \\
\mu_{2}=\nu_{2}-\frac{1}{12} \\
\mu_{3}=\nu_{3} \\
\mu_{4}=\nu_{4}-\frac{1}{2} \nu_{2}+\frac{7}{2} \frac{7}{40}=\nu_{4}-\frac{1}{2} \mu_{2}-\frac{1}{80}
\end{array}\right\}
$$

Using accented $\nu$ 's to denote the moments of the grouped observations about other ordinates than the centroid vertical, the relations (1) and (2) above hold for $\nu_{n}, \nu_{n}{ }^{\prime}$, etc. There are thus three stages in the evaluation of $\mu_{1}, \mu_{2}$, etc.

(1) Evaluate $\nu_{n}^{\prime}$ for $n=1,2,3$, etc. about any convenient ordinate.

(2) Transform to the centroid vertical by using equations (1) or (2), so obtaining $\nu_{2}, \nu_{3}$, etc. $\quad \nu_{1}=0$.

(3) From these values deduce $\mu_{2}, \mu_{3}, \mu_{4}$, etc. by the use of equations (5) above. It should be remembered that, referred to the centroid vertical, $\mu_{1}=\nu_{1}=0$.

The solution of equations (4) for $a, b_{0}, b_{1}, b_{2}$ is quite straightforward. The resulting form of equation (3) is

$$
-\frac{1}{y} \frac{d y}{d x}=\frac{x+\frac{\mu_{3}\left(\mu_{4}+3 \mu_{2}{ }^{2}\right)}{10 \mu_{2} \mu_{4}-18 \mu_{2}{ }^{3}-12 \mu_{3}{ }^{2}}}{\frac{\mu_{2}\left(4 \mu_{2} \mu_{4}-3 \mu_{3}{ }^{2}\right)+\mu_{3}\left(\mu_{4}+3 \mu_{2}{ }^{2}\right) x+\left(2 \mu_{2} \mu_{4}-3 \mu_{3}{ }^{2}-6 \mu_{2}{ }^{3}\right) x^{2}}{10 \mu_{2} \mu_{4}-18 \mu_{2}{ }^{3}-12 \mu_{3}{ }^{2}}} .
$$

* W. F. Sheppard, Proc. L.M.S. xxix, p. 353; see also, Karl Pearson, Biometrika, III, p. 308. 
If in this last form we substitute

$$
\beta_{1}=\frac{\mu_{3}^{2}}{\mu_{2}{ }^{3}}, \quad \beta_{2}=\frac{\mu_{4}}{\mu_{2}^{2}}, \quad \sigma=\sqrt{\mu_{2}},
$$

we obtain the equation in the form

$-\frac{1}{y} \frac{d y}{d x}$

$$
x+\frac{\sigma \sqrt{\beta_{1}}}{2} \cdot \frac{\beta_{2}+3}{5 \beta_{2}-6 \beta_{1}-9}
$$

$$
=\frac{\sigma^{2}\left\{\frac{4 \beta_{2}-3 \beta_{1}}{10 \beta_{2}-12 \beta_{1}-18}+\frac{\sqrt{\beta_{1}}}{2} \frac{\beta_{2}+3}{5 \beta_{2}-6 \beta_{1}-9} \frac{x}{\sigma}+\frac{2 \beta_{2}-3 \beta_{1}-6}{10 \beta_{2}-12 \beta_{1}-18}\left(\frac{x}{\sigma}\right)^{2}\right\}}{\text {. }}
$$

This equation is referred to the mean as origin. The mode is obtained by making the numerator in the R. H. S. zero. It follows that the skewness of the curve is

$$
\frac{\sqrt{\beta_{1}}}{2} \cdot \frac{\beta_{2}+3}{5 \beta_{2}-6 \beta_{1}-9} .
$$

The curve is symmetrical when $\beta_{1}=0$.

The form of the curve is fixed by the nature of the roots of the equation

$$
b_{0}+b_{1} x+b_{2} x^{2}=0
$$

i.e. by the value of $b_{1}{ }^{2}-4 b_{0} b_{2}$; or by $\frac{b_{1}{ }^{2}}{4 b_{0} b_{2}}$.

Substituting the values of $b_{0}, b_{1}, b_{2}$ derived above, we find

$$
\frac{b_{1}^{2}}{4 b_{0} b_{2}}=\frac{\beta_{1}\left(\beta_{2}+3\right)^{2}}{4\left(2 \beta_{2}-3 \beta_{1}-6\right)\left(4 \beta_{2}-3 \beta_{1}\right)}
$$

The value of this function of the moments fixes the nature of the curve of frequencies. Hence it is known as the criterion.

\section{Integration of the Differential Equation.}

Returning to the general differential equation

$$
\frac{1}{y} \frac{d y}{d x}=\frac{x+a}{b_{0}+b_{1} x+b_{2} x^{2}}
$$

we find that seven different types of curves can be derived from it according to the nature of the constants involved. 
I. If

then

or

$$
b_{1}=b_{2}=0 \text {, }
$$

$$
\frac{1}{y} \frac{d y}{d x}=\frac{x+a}{b_{0}}
$$

$$
\begin{gathered}
\log y=\frac{x^{2}}{2 b_{0}}+\frac{a x}{b_{0}}+\text { const. } \\
y=y_{0} e^{\frac{(x+a)^{2}}{2 b_{0}}},
\end{gathered}
$$

where $b_{0}$ must be negative.

This is Gauss's Normal Error curve, with the mean at $x=-a$. In this case the criterion is zero, $\beta_{1}$ being zero.

II. If $b_{2}=0$, then

$$
\frac{1}{y} \frac{d y}{d x}=\frac{x+a}{b_{0}+b_{1} x}=\frac{1}{b_{1}}+\frac{a-\frac{b_{0}}{b_{1}}}{b_{0}+b_{1} x},
$$

and $\quad \log y=\frac{x}{b_{1}}+\frac{1}{b_{1}}\left(a-\frac{b_{0}}{b_{1}}\right) \log \left(b_{0}+b_{1} x\right)+$ constant,

$$
y=y_{0}^{\prime}\left(b_{0}+b_{1} x\right)^{\frac{a b_{1}-b_{0}}{b_{1}^{2}}} e^{\frac{x}{b_{1}}}=y_{0}^{\prime \prime} e^{\frac{x+a}{b_{1}}}\left\{b_{1}(x+a)+b_{0}-a b_{1}\right\}^{\frac{a b_{1}-b_{0}}{b_{1}^{2}}} \text {. }
$$

Removing the origin to $x=-a$, and writing

$$
l=\frac{b_{0}}{b_{1}}-a, \quad \gamma=-\frac{1}{b_{1}},
$$

we obtain the equation in the form

$$
y=y_{0}\left(1+\frac{x}{l}\right)^{\gamma l} e^{-\gamma x} \text {. }
$$

The curve is limited in one direction, and is skew, the origin being at the mode.

The value of the criterion in this case is $\infty$, this value being due to the relation

$$
2 \beta_{2}-3 \beta_{1}-6=0 \text {. }
$$

III. When the roots of $b_{0}+b_{1} x+b_{2} x^{2}=0$ are real and of opposite sign, the equation may be written

$$
\begin{aligned}
& \frac{b_{0}}{y} \frac{d y}{d x}=\frac{x+a}{\left(x+c_{1}\right)\left(x-c_{2}\right)}=\frac{c_{1}-a}{c_{1}+c_{2}} \frac{1}{x+c_{1}}+\frac{c_{2}+a}{c_{1}+c_{2}} \frac{1}{x-c_{2}}, \\
\therefore \quad & b_{0} \log y=\frac{c_{1}-a}{c_{1}+c_{2}} \log \left(x+c_{1}\right)+\frac{c_{2}+a}{c_{1}+c_{3}} \log \left(x-c_{2}\right)+\text { constant. }
\end{aligned}
$$


Removing the origin to $x=-a$, and writing

$$
a_{1}=c_{1}-a, \quad a_{2}=c_{2}+a, \quad \nu=\frac{1}{b_{0}\left(c_{1}+c_{2}\right)},
$$

this equation becomes

$$
\begin{gathered}
\log y=\nu a_{1} \log \left(x+a_{1}\right)+\nu a_{2} \log \left(x-a_{2}\right)+\text { constant } \\
y=y_{0}\left(1+\frac{x}{a_{1}}\right)^{v a_{1}}\left(1-\frac{x}{a_{2}}\right)^{v a_{2}}
\end{gathered}
$$

or

the origin being at the mode.

The criterion is negative. The curve is limited in both directions, and is skew.

IV. When the roots of $b_{0}+b_{1} x+b_{2} x^{2}=0$ are real, of opposite sign, but equal in magnitude, we should have $c_{1}=c_{2}$ in III, and consequently $a_{i}=a_{2}$.

The resulting equation is

$$
y=y_{0}\left(1-\frac{x^{2}}{a^{2}}\right)^{v a}
$$

referred to the mode as origin.

This is a symmetrical curve, limited in both directions.

The nature of the roots requires that $b_{1}=0$, and therefore $\beta_{1}=0$.

Hence the criterion is zero.

$\mathrm{V}$. When the equation $b_{0}+b_{1} x+b_{2} x^{2}=0$ has real and equal roots,

$$
\frac{1}{y} \frac{d y}{d x}=\frac{x+a}{b_{2}\left(x+\frac{b_{1}}{2 b_{2}}\right)^{2}}=\frac{1}{b_{2}} \frac{1}{x+\frac{b_{1}}{2 b_{2}}}+\frac{1}{b_{2}} \frac{a-\frac{b_{1}}{2 b_{2}}}{\left(x+\frac{b_{1}}{2 b_{2}}\right)^{2}},
$$

which on integrating yields

$$
\log y=-\frac{1}{b_{2}}\left(a-\frac{b_{1}}{2 b_{2}}\right) \frac{1}{x+\frac{b_{1}}{2 b_{2}}}+\frac{1}{b_{2}} \log \left(x+\frac{b_{1}}{2 b_{2}}\right)+\text { constant. }
$$

Removing the origin to $x=-\frac{b_{1}}{2 b_{2}}$, and writing

$$
p=\frac{1}{b_{2}}, \quad \gamma=\frac{1}{b_{2}}\left(a-\frac{b_{1}}{2 b_{2}}\right),
$$


we may write this equation in the form

or

$$
\begin{gathered}
\log y=-\frac{\gamma}{x}+p \log x+\text { constant, } \\
y=y_{0} x^{p} e^{-\frac{\gamma}{x}} .
\end{gathered}
$$

This is a skew curve, with a limited range in one direction. The criterion is unity.

VI. When the roots of the equation $b_{0}+b_{1} x+b_{2} x^{2}=0$ are real and unequal, and of the same sign.

Let the roots be $c_{1}$ and $c_{2}$. Then, as in type III,

$$
b_{0} \log y=\frac{c_{1}+a}{c_{1}-c_{2}} \log \left(x-c_{1}\right)-\frac{c_{2}+a}{c_{1}-c_{2}} \log \left(x-c_{2}\right) .
$$

Changing the origin to $x=c_{1}$, we may write

$$
y=y_{0} x^{m_{2}}(x-c)^{-m_{2}} \text {. }
$$

The criterion is positive and greater than unity.

VII. When the roots are complex, the differential equation may be simplified slightly by transferring the origin to

$$
x=-\frac{b_{1}}{2 b_{2}}
$$

and putting

$$
c=a-\frac{b_{1}}{2 b_{2}}
$$

and

It then reads

$$
d^{2}=\frac{b_{0}}{b_{2}}-\frac{b_{1}^{2}}{4 b_{2}^{2}} \text {. }
$$

$$
\frac{1}{y} \frac{d y}{d x}=\frac{x+c}{b_{2}\left(x^{2}+d^{2}\right)}=\frac{1}{b_{2}} \frac{x}{x^{2}+d^{2}}+\frac{c}{b_{2}} \frac{1}{x^{2}+d^{2}},
$$

whence $\quad \log y=\frac{1}{2 b_{2}} \log \left(x^{2}+d^{2}\right)+\frac{c}{b_{2} d} \tan ^{-1} \frac{x}{d}+$ constant,

or

$$
\begin{aligned}
& y=y_{0}^{\prime}\left(x^{2}+d^{2}\right)^{\frac{1}{2 b_{2}}} e^{\frac{c}{b_{2} d} \tan ^{-1} \frac{x}{d}}, \\
& y=y_{0} \frac{e^{-\nu \tan ^{-1} \frac{x}{d}}}{\left(1+\frac{x^{2}}{d^{2}}\right)^{m}} .
\end{aligned}
$$

This is a skew curve with unlimited range. The criterion is positive but less than unity. 
The value of the criterion is clearly sufficient to determine which of the seven types given above will best represent the statistical data under consideration. When the criterion is negative the curve is always of type III, when it is positive but less than 1, the curve is of type VII, and when it is positive and greater than 1, the curve is of type VI. These include the majority of cases that can arise, but at the points where the curve changes from one type to another, a transition curve can be used. Thus when the criterion is $\infty$, a curve of type II can be used, and when the criterion is unity the curve is of type $\mathrm{V}$. When the criterion is zero, on account of $\beta_{1}$ being zero, two types, I and IV, are possible. The former is the normal curve of errors, for which $\mu_{4}=3 \mu_{2}{ }^{2}$ (see page 56) and so $\beta_{z}=3$. Thus when $\beta_{1}=0$ and the criterion vanishes, the curve is of type I if $\beta_{2}=3$, and of type IV if $\beta_{2}$ has any value other than 3.

For a detailed application of the above types of curves to the adjustment of statistical data, the reader is referred to FrequencyCurves and Correlation by W. Palin Elderton.

65. Use of the series

$$
y=A_{0} \phi(x)+A_{3} \phi^{\prime \prime \prime}(x)+A_{4} \phi^{\mathrm{iv}}(x)+\text { etc. }
$$

In this series $A_{0}, A_{3}, A_{4}$, etc. are constants, and $\phi(x)$ is given by

$$
\phi(x)=\frac{1}{\sigma \sqrt{2 \pi}} e^{\frac{-(x-b)^{2}}{2 \sigma^{2}}}, \quad \phi^{\prime \prime \prime}(x)=\frac{d^{3}}{d x^{3}} \phi(x), \text { etc., }
$$

$\sigma$ being the S.D. of the distribution.

The use of this form of frequency curve has been proposed by Thiele*, Edgeworth $\uparrow$, and Charlier+. It should be noted that the first term of the series gives the normal error curve. The next term introduces skewness into the curve, while the effect of the third term is symmetrical. Charlier, in the first paper referred to, developed this form of the error law from the hypothesis that an error is made up of a large number of small errors, each of which has its own error law. Edgeworth made considerable use of a functional form which involved only the first two terms in the series.

* Theory of Observations, London, 1903.

+ Camb. Phil. Trans., Vol. xx, pp. 36-65, 113-141.

‡Arkiv för Matematik, Vol. 1r, Stockholm, 1905, "Über das Fehlergesetz"; Lunds Meddelanden, 1906, "Researches into the Theory of Probability."

B. 0. 
Charlier fits the curve to the observations by the method of moments. With the notation of the present chapter, this gives

$$
\begin{aligned}
b & =\mu_{1}^{\prime} \quad \sigma^{2}=\mu_{2} \\
A_{0} & =1 \\
3 ! A_{3} & =-\mu_{3} \\
4 ! A_{4} & =\mu_{4}-3 \sigma^{4} \\
5 ! A_{5} & =-\mu_{5}+10 \sigma^{2} \mu_{3} \\
6 ! A_{6} & =\mu_{6}-15 \sigma^{2} \mu_{4}+15 \sigma^{6} .
\end{aligned}
$$

It is thus not difficult to fit a curve to any given series of observations.

66. Other forms of possible frequency curves might be suggested, and with most of these it is not difficult to fit theory to fact. The difficulty lies rather in finding some means of estimating the relative values of the different laws of presumptive errors. We ask in vain for a fixed rule by which the most important and trustworthy forms can be selected. The difficulty is scarcely minimised by the fact that it is often possible to obtain fairly good representations of a given set of observations by two curves whose functional forms differ very widely. Thus Elderton, in his excellent book on Frequency-Curves and Correlation, shows that a certain series of observations can be almost equally well represented by the two forms

$$
y=y_{0}\left(1+\frac{x^{2}}{(13 \cdot 39152)^{2}}\right) e^{-4 \cdot 4504 \tan ^{-1} \frac{x}{13 \cdot 39152}},
$$

and $y=4302\left[2 \cdot 127818 \phi(x)+\cdot 012208 \times(2 \cdot 127818)^{4} \phi^{\prime \prime \prime}(x)\right.$

$$
\left.+\cdot 007079 \times(2 \cdot 127818)^{5} \phi^{\text {iv }}(x)\right],
$$

while another series of observations can be represented by the two forms

$$
y=462.57\left(1-\frac{x^{2}}{(4 \cdot 543079)^{2}}\right)^{4 \cdot 141766},
$$

and $y=1244 \cdot 4\left[\sigma \phi(x)-\cdot 0081 \sigma^{4} \phi^{\prime \prime \prime}(x)-\cdot 01882 \sigma^{5} \phi^{\text {iv }}(x)\right]$, where $\quad \sigma^{2}=1.829172$. 
All that seems at all definite is that with the series

$$
y=A_{0} \phi(x)+A_{3} \phi^{\prime \prime \prime}(x)+A_{4} \phi^{\text {iv }}(x)+\text { etc. }
$$

it is difficult to graduate a very skew distribution, or one that rises very rapidly from the axis. For, to do so would involve the use of a large number of terms of the series, so involving the higher moments, whose probable errors are considerably greater than those of the lower moments. In such cases it is better to adhere to Pearson's family of curves. The straightforward use of the criterion will lead to the type of curve which should give the best fit. 


\section{CHAPTER $\mathrm{X}$.}

\section{CORRELATION}

67. In the preceding chapters we have only considered frequency distributions due to a single variable, or to more than one independent variable. In the present chapter we shall consider the case where the variables are correlated. The method may be illustrated by a simple example. The first two columns of the table on p. 157 below give the orbital period and duration

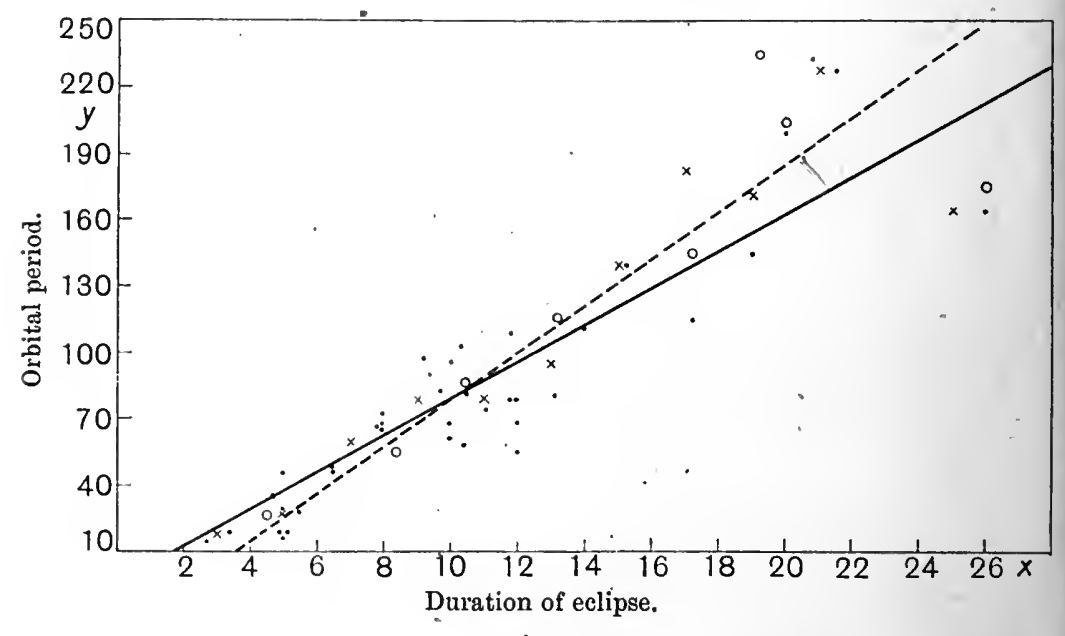

Fig. 9.

of eclipse of 38 Algol stars*. These are represented diagrammatically in figure 9 . The horizontal scale $(x)$ represents the duration of eclipse in hours, and the vertical scale $(y)$ represents the orbital period in hours. Each dot in the diagram represents

* Father Stein, Monthly Notices, R.A.S., Vol. Lxrx, p. 450. 
one star. Such a diagram may be conveniently callerl a "scatter diagram."

The points in the scatter diagram are roughly grouped about a straight line, showing, as might have been anticipated a priori, that the duration of eclipse generally increases as the orbital period increases. The total range of duration of eclipse is subdivided into ranges $2^{\mathrm{h}}-4^{\mathrm{h}}, 4^{\mathrm{h}}-6^{\mathrm{h}}$, etc. The mean orbital period for all the stars in each of these intervals is calculated, and represented in the diagram by means of $a \times$ placel at the middle of the range, e.g. the mean orbital period of stars whose duration of eclipse is between $10^{\mathrm{h}}$ and $12^{\mathrm{h}}$ is $79^{\mathrm{h}}$, and this mean is represented in the diagram by $\mathrm{a} \times$ at $11^{\mathrm{h}}, 79^{\mathrm{h}}$. Similarly the mean duration of eclipse for stars whose orbital periods are between $10^{\mathrm{h}}$ and $40^{\mathrm{h}}$, between $40^{\mathrm{h}}$ and $70^{\mathrm{h}}$, etc., are represented in the diagram by small circles.

A straight line is drawn to fit the series of crosses as accurately as possible. In this particular instance the crosses do not lie accurately on the straight line, but lie irregularly on either side of it. This straight line is called the line of regression of $y$ on $x$. Its ordinate gives the mean value of $y$, which we may expect to find associated with a given value of $x$. Similarly the straight line which fits most accurately the series of small circles is called the line of regression of $x$ on $y$. Its abscissa gives the mean value of $x$ corresponding to a given value of $y$. It should be noted that the two lines of regression do not coincide, though the angle between them is small. When the means lie fairly accurately on a straight line, the regression is said to be linear. But it may happen that the means lie, not on a straight line, but on a welldefined curve. The curve is called the curve of regression, and the regression is then defined as non-linear. Such a curve would be obtained if a number of observations of the volume $(v)$ and the pressure $(p)$ of a given mass of gas at a constant temperature were represented by a scatter diagram. The points in the seatter diagram would lie closely about a rectangular hyperbola, whose equation is $p v=$ constant. Even in cases where there is a clearly defined non-linear curve of regression, the straight lines which fit most closely the series of means are called the lines of regression. 
Generally speaking, it is only in dealing with isolated physical phenomena, in which the conditions of observation can be completely controlled, that we shall find a clearly defined functional relation between the two variables considered. When the factors which vary are complex and not controllable by the observer, the curve of regression does not as a rule indicate a simple functional relationship between the two characters considered. The complete problem of the statistician, which is to find formulae which will represent with sufficient accuracy the form of the curve of regression, is not in general capable of solution. But since the majority of the problems of the practical statistician relate solely to averages, it is sufficient in many cases to be able to state whether, on an average, there is a tendency for high values of one of the characters to be associated with high values (or low values) of the other. If possible it is also desirable to find how great a divergence of one character from its mean value is associated with a unit divergence of the other from its mean value; and also how closely this relation is usually fulfilled. These questions can be largely decided by fitting a straight line to the series of means obtained as in figure 9, i.e. by drawing the lines of regression.

68. As an alternative to the scatter diagram, we might represent the frequency distribution of two variables by means of a table of double entry, or a contingency table. The following contingency table represents the material in the scatter diagram of figure 9 .

Each row in this table gives the frequency distribution of the duration of eclipse for a given range of orbital period, while each column gives the frequency distribution of the orbital period for a given range of duration of eclipse. As the colunns and rows are only distinguished by the accidental circumstance that one runs vertically and the other horizontally, the word "array" is used to denote either a row or a column.

The choice of class-intervals is to a large extent arbitrary, and in general any interval which happens to be convenient may be selected. When this choice has been made, the contingency table can be completed in a number of ways. If a scatter diagram such as that of figure 9 has been made, the class- 
intervals may be ruled in the diagram, and the number of dots in each compartment may then be counted. If such a scatter diagram is not available, a form such as that shown below should be ruled on a large sheet of paper, with the class-intervals headed as in the fimal table. Each observation can be representerl in this table by a cross in the corresponding compartment. The sum

Duration of eclipse in hours.

\begin{tabular}{|c|c|c|c|c|c|c|c|c|c|c|c|c|c|}
\hline & $2-$ & $4-$ & $6-$ & $8-$ & $10-$ & $12-$ & $14-$ & $16-$ & 18 & 20 & $22-$ & $24-$ & Total \\
\hline $10-$ & 2 & 6 & - & - & - & - & - & - & - & - & - & - & 8 \\
\hline $40-$ & - & 1 & 3 & 2 & 3 & 2 & - & - & - & - & - & - & 11 \\
\hline $70-$ & - & - & - & 3 & 4 & 2 & - & - & - & - & - & - & 9 \\
\hline $100-$ & - & - & - & - & 2 & - & I & 1 & - & - & - & - & 4 \\
\hline $130-$ & - & - & - & - & - & - & 1 & - & 1 & - & - & - & 2 \\
\hline $160-$ & - & - & - & - & - & - & - & - & - & - & - & 1 & 1 \\
\hline $190-$ & - & - & - & - & - & - & - & - & - & 1 & - & - & 1 \\
\hline $220-$ & - & - & - & - & - & - & - & 1 & - & 1 & - & - & 2 \\
\hline Total & 2 & 7 & 3 & 5 & 9 & 4 & 2 & 2 & 1 & 2 & - & 1 & 38 \\
\hline
\end{tabular}

of the crosses in a compartment is then entered in the corresponding compartment of the final table. It should be noted that any compartment may contain halves and even quarters of a frequency. For if an observation falls exactly on the dividing line between two compartments, it is counted as a half in each of those compartments; and if an observation falls exactly at the common angular point of four compartments, it is counted as a quarter in each of the four compartments.

When there is a functional relation between the two characters considered, as will often happen in the problems of the physicist or chemist, there will be entries in only a few compartments of the contingency table. The way in which the entries are grouped will. afford some idea of the relationship between the two variable characters. Thus in the contingency table above, the entries run diagonally across the table, showing distinct correlation between the two characters considered. 
When the means represented by crosses (or circles) in the scatter diagram (see tigure 9) lie accurately on a strdight line, the two variables considered are connected by a linear relation, and are said to be completely correlated. In practice, however, this seldom occurs, and the means lie irregularly about a straight line. The straight line which best fits the series of means might be drawn by a simple graphical method, say by means of a stretched thread moved about until as many of the means lie on one side as on the other. But such a method would generally allow of our drawing a number of straight lines, any one of which would apparently be as good a fit as any other. It is therefore necessary to adopt some standard method of drawing what shall be regarded as the best-fitting straight line. The method commonly adopted is based on the theory of least squares, but it should be remembered that this step is arbitrary, and that other methods might be suggested which would yield equally good results. The method of moments yields precisely the same results as the least squares method*.

69. The total range of $x$-variation is divided into a con-

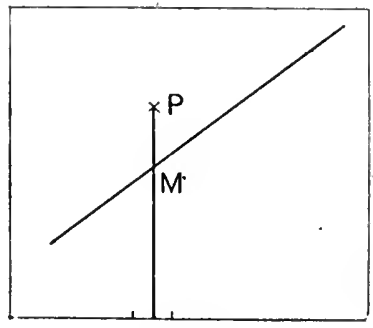

Fig. 10.

venient number of intervals. Let there be $n_{x}$ observations, i.e. $n_{x} y$ 's, in any one of these intervals, and let the mean of these $y$ 's be represented by the ordinate of the point $P$ in figure 10. A number of points such as $P$ will be represented in the completed figure, each point corresponding to the mean of all the $y^{\prime}$ s in one $x$-interval. The curve of regression passes through all these points. We now have to draw the straight

* Elderton, Frequency-Curves and Correlation, p. 114. 
line which shall best fit all these points, or, in other words, we have to find the equation of the line of regression of $y$ on $x$.

Referred to axes through the means of $x$ and $y$, lot the equation of the line of regression be

$$
Y=a+b X \text {. }
$$

Let the ordinate of $P$ meet the line of regression at the point $M$. Then the usual least squares method of adjustment is to make $\Sigma n_{x} P M^{2}$ a minimum. Let the ordinate of $P$ be $y_{x}$, and of $M, Y$. Then we have to make

$$
V=\Sigma n_{x}\left(Y-y_{x}\right)^{2}
$$

a minimum, where the summation extends to all the $x$-intervals.

Since $Y=a+b X$ for the point $M$,

$$
\begin{aligned}
V & =\Sigma n_{x}\left\{(a+b X)-y_{x}\right\}^{2} \\
& =\Sigma n_{x}\left\{a^{2}+b^{2} X^{2}+2 a b X+y_{x}{ }^{2}-2 a y_{x}-2 b X y_{x}\right\} .
\end{aligned}
$$

But since the origin is at the mean of $x$ and $y$,

$$
\Sigma n_{x} y_{x}=0, \quad \Sigma n_{x} X=0,
$$

and therefore

$$
V=\Sigma n_{x}\left\{a^{2}+b^{2} X^{2}+y_{x^{2}}-2 b X y_{x}\right\}
$$

This last form of $V$ clearly demands $\iota=0$ for ${ }^{\circ i t s}$ minimum value. Differentiating with respect to $b$,

and

$$
2 b \Sigma n_{x} X^{2}-2 \Sigma n_{x} X y_{x}=0
$$

$$
b=\frac{\sum n_{x} X y_{x}}{\sum n_{x} X^{2}}=\frac{\Sigma x y}{\Sigma x^{2}},
$$

where, in the last fractional form, the summation extends to all pairs of associated values of $x$ and $y$. If $\sigma_{1}, \sigma_{2}$ be the standard deviations of $x$ and $y$ respectively,

$$
\Sigma x^{2}=N \sigma_{1}^{2}, \quad \Sigma y^{2}=\dot{N} \sigma_{2}^{2} \text {. }
$$

Let

$$
\Sigma_{x y}=N r \sigma_{1} \sigma_{2} \text {. }
$$

Then $b=r \frac{\sigma_{2}}{\sigma_{1}}$, and the equation of the line of regression of $y$ on $x$ is

or

$$
\begin{aligned}
y & =r \cdot \frac{\sigma_{2}}{\sigma_{1}} x, \\
\frac{y}{\sigma_{2}} & =r \frac{x}{\sigma_{1}} .
\end{aligned}
$$


Similarly the line of regression of $x$ on $y$ may be shown to have the equation

or

$$
\begin{aligned}
x & =r \frac{\sigma_{1}}{\sigma_{2}} y, \\
\frac{x}{\sigma_{1}} & =r \frac{y}{\sigma_{2}} .
\end{aligned}
$$

If the measures $x, y$ be referred to some other zero than the mean, and $\bar{x}, \bar{y}$ be their mean values, the equations of the lines of regression given above are changed into

$$
\begin{aligned}
& y-\bar{y}=r \frac{\sigma_{2}}{\sigma_{1}}(x-\bar{x}), \\
& \dot{x}-\bar{x}=r \frac{\sigma_{1}}{\sigma_{2}}(y-\bar{y}) .
\end{aligned}
$$

In these equations $r$ is a quantity defined by the equation

$$
\Sigma x y=N r \sigma_{1} \sigma_{2} \text {. }
$$

This quantity $r$ is called the coefficient of correlation.

70. Returning to the function $V$ defined above, we may now write

$$
V=\Sigma n_{x}\left(b X-y_{x}\right)^{2}
$$

where the summation extends to all the $x$-arrays,

or

$$
V=\Sigma(b x-y)^{2}=\Sigma\left(r \frac{\sigma_{2}}{\sigma_{1}} x-y\right)^{2},
$$

where the summation extends to all the pairs of associated deviations.

Hence

$$
\begin{aligned}
V & =\frac{r^{2} \sigma_{2}{ }^{2}}{\sigma_{1}{ }^{2}} \Sigma x^{2}-\frac{2 r \sigma_{2}}{\sigma_{1}} \Sigma x y+\Sigma y^{2} \\
& =N\left\{\frac{r^{2} \sigma_{2}{ }^{2}}{\sigma_{1}{ }^{3}} \sigma_{1}^{2}-2 r^{2} \sigma_{2}{ }^{2}+\sigma_{2}{ }^{2}\right\} \\
& =N \sigma_{2}{ }^{2}\left(1-r^{2}\right) .
\end{aligned}
$$

If $s_{2}$ be the M.S.E. due to taking the value of $y$ given by the line of regression

$$
y=\frac{r \sigma_{2}}{\sigma_{1}} x
$$

instead of the measured deviation of $y$, then

and

$$
V=N s_{2}{ }^{2}=N \sigma_{2}{ }^{2}\left(1-r^{2}\right) \text {, }
$$

$$
s_{2}=\dot{\sigma}_{2}\left(1-r^{2}\right)^{\frac{1}{2}} \text {. }
$$


Similarly if $s_{1}$ be the M.S.E. of $x$ derived from the equation

$$
x=\frac{r \sigma_{1}}{\sigma_{2}} y,
$$

then

$$
s_{1}=\sigma_{1}\left(1-r^{2}\right)^{\frac{1}{2}} \text {. }
$$

If $v=1$, then $V=0$, and $s_{1}=s_{2}=0$. And since

$$
V=\Sigma(b x-y)^{2}
$$

it follows that when

$$
\begin{gathered}
r=1, \\
b x-y=0
\end{gathered}
$$

for each pair of associated deviations $x, y$. In other words, a linear relation

$$
\frac{x}{\sigma_{1}}-\frac{y}{\sigma_{2}}=0
$$

is then rigorously satisfied by all pairs of values of $x, y$.

If the coefficient $r$ only differs slightly from unity, the points in the scatter diagram are closely grouped about a straight line. The two lines of regression (which coincide when $r=1$ ) are then inclined to one another at a small angle.

If $r$ be small the angle between the lines of regression is large, and when $r=0$, the lines are at right angles, their equations being $y=0, x=0$. In the case where $r$ is small, the M.S.E. of $y$ caused by our adopting the linear relation $y=\frac{r \sigma_{2}}{\sigma_{1}} x$ between the two variables instead of using the original observations, defined above as $s_{2}$, is nearly as great as $\sigma_{2}$. Or in other words, if we want to find the value of $y$ corresponding to a given value of $x$, the value $\frac{r \sigma_{2}}{\sigma_{1}} x$ is only a very slight improvement on the mean value of all the $y$ 's in the case where $r$ is small.

When $r$ is small, there is only very slight correlation between the two variable characters considered, and it seems doubtful whether any serious meaning can be attached to values of $r$ which are less than 5 .

When $r=0$, there is apparently no correlation between $x$ and $y$, and the lines of regression are at right angles to one another. In the next diagram (fig. 11), however, is shown an extreme case 
where $r=0$, while the variables are connected by a clearly marked relation. It is therefore not safe to assume a complete absence of correlation in cases where the coefficient $r$ is very small. The evaluation of the correlation ratio, which will be discussed later, affords a better test of correlation in such cases.

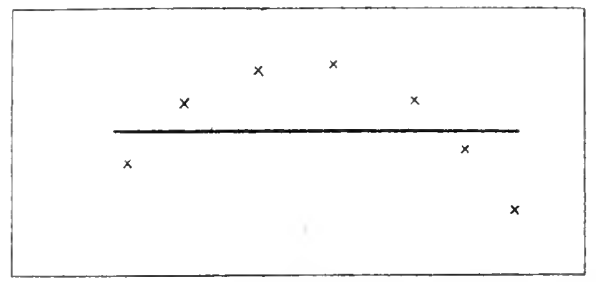

Fig. 11. Curve of regression of $y$ on $x$, together with the corresponding line of regression.

\section{Evaluation of $\mathrm{r}$ for the material of figure $\mathbf{9}$.}

In the following table we shall evaluate $r$ for the material which has been represented diagrammatically in figure 9 . The first column gives the orbital period in hours, and the second column the duration of eclipse in hours, for 38 stars. The means of these columns are evaluated. In the third and fourth columns are given the deviations from these means of the orbital period and duration of eclipse of each star. These are the quantities which we have called $y$, and $x$, respectively, in the preceding discussion. The remainder of the table is self-explanatory.

$$
\begin{gathered}
r \sigma_{1} \sigma_{2}=257 \cdot 54 ; \\
\therefore r=\frac{257.54}{55.6 \times 5.26}=881, \\
\frac{r \sigma_{2}}{\sigma_{1}}=\frac{881 \times 5.5 \cdot 6}{5 \cdot 26}=8.325, \\
\frac{r \sigma_{1}}{\sigma_{2}}=\frac{881 \times 5.26}{55.6}=\cdot 093 .
\end{gathered}
$$

The corresponding lines of regression are

$$
Y-82 \cdot 7=8 \cdot 325(X-10 \cdot 4) \text {, }
$$

or

$$
Y=8 \cdot 325 X-3.88
$$

and

$$
X-10 \cdot 4=093(Y-82 \cdot 7) \text {, }
$$

or

$$
X=\cdot 093 Y+2 \cdot 7
$$




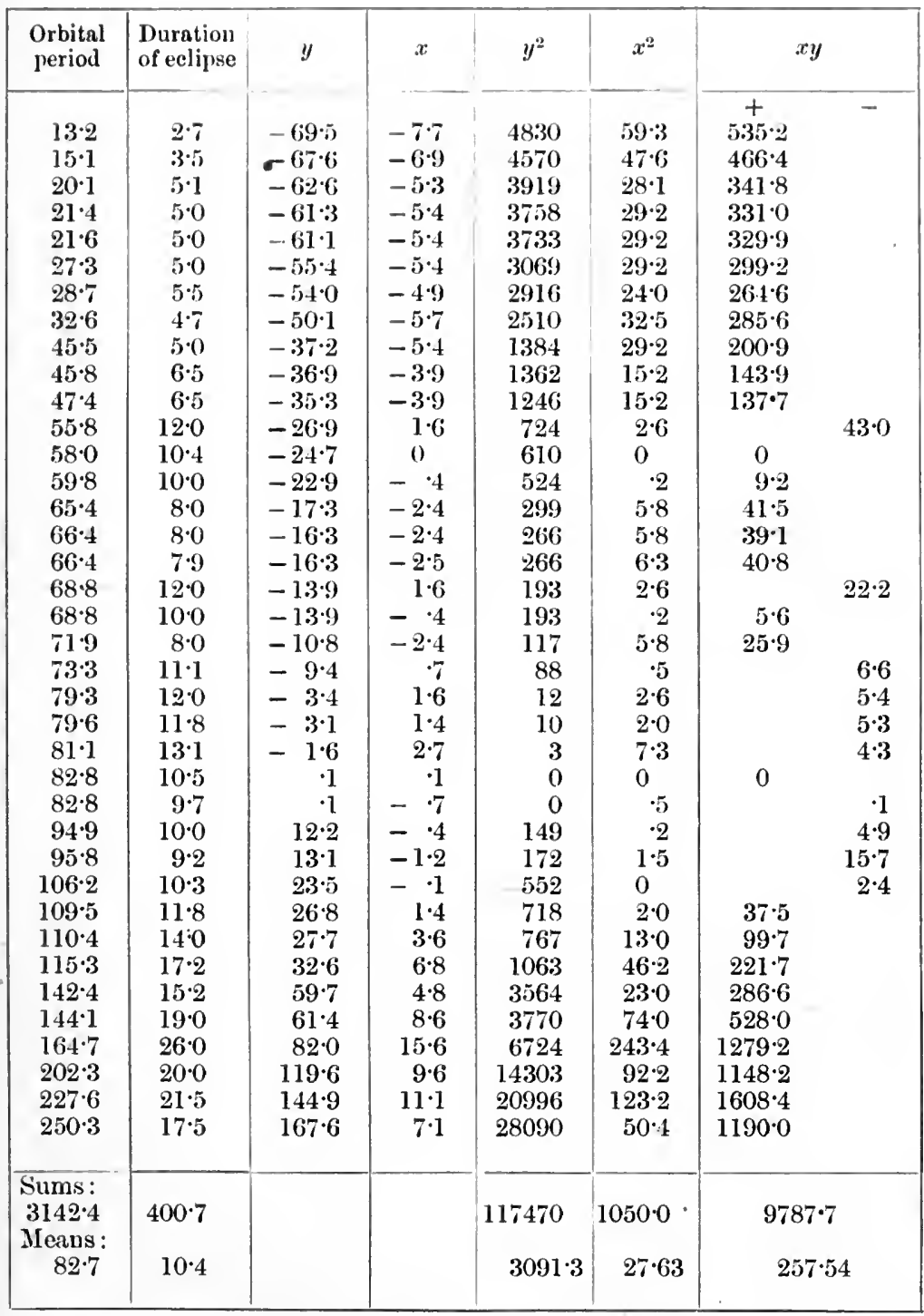

$\sigma_{2}=55 \cdot 6 \quad \sigma_{1}=5 \cdot 26$

The two straight lines are represented in figure 9 by the continuous and dotted lines respectively.

The mean square error in $y$ due to assuming the relation $A$ is $55 \cdot 6 \sqrt{1-(\cdot 881)^{2}}$ hours $=26 \cdot 4$, hours, 
and the mean square error in $x$ due to assuming the relation $B$ is $5 \cdot 26 \sqrt{1-(\cdot \overline{881})^{2}}$ hours $=2 \cdot 5$ hours.

\section{Calculation of $\mathbf{r}$ from the Contingency Table.}

When the number of observations is large, the method of calculating $r$ used in the preceding table becomes extremely laborious. It is then better to calculate $r$ from the contingency table. To illustrate the method, we shall evaluate $r$ from the contingency table of page 151 .

Duration of eclipse.

\begin{tabular}{|c|c|c|c|c|c|c|c|c|c|c|c|c|c|}
\hline & 2 & $4-$ & $6-$ & $8-$ & $10-$ & 12 & $14-$ & $16-$ & $18-$ & 20 & $22-$ & 24 & Totals \\
\hline $10-$ & $8^{2}$ & ${ }_{6} 6$ & & & & & & & & & & & 8 \\
\hline $40-$ & & ${ }_{3} 1$ & ${ }_{2}^{3}$ & 12 & ${ }_{0} 3$ & 12 & & & & & & & 11 \\
\hline $70-$ & & & & $0_{0}^{3}$ & $0^{4}$ & $0^{2}$ & & & & & & & 9 \\
\hline $100-$ & & & & & $0_{0}^{2}$ & & $2^{1}$ & ${ }_{3} 1$ & & & & & 4 \\
\hline $130-$ & & & & & & & $4^{1}$ & & $8_{8}^{1}$ & & & & 2 \\
\hline $160-$ & & & & & & & & & & & & ${ }_{21}^{1}$ & 1 \\
\hline $190-$ & & & & & & & & & & ${ }_{20}^{1}$ & & & 1 \\
\hline $220-$ & & & & & & & & ${ }_{15} 1$ & & ${ }_{25}^{1}$ & & & 2 \\
\hline Totals & 2 & 7 & 3 & 5 & 9 & 4 & 2 & 2 & 1 & 2 & - & 1 & 38 \\
\hline
\end{tabular}

The above table is a repetition of the table of page 151, with the arrays which contain the means of the two variables marked by thick lines. The midpoints of these two arrays are taken as zeros for the coordinates, and the class-intervals are taken as units in terms of which the s.D.'s of $x$ and $y$ are to be expressed. The following tables give the calculation.

Each of the tables $(a)$ and $(b)$ gives the calculation of the mean and of the S.D. for one of the variables. For still greater accuracy, we might apply Sheppard's corrections to the values of $\sigma_{1}$ and $\sigma_{2}$. 
(a) For $x$.

\begin{tabular}{|c|c|c|c|}
\hline Sum & Factor & 1st product & 2nd product \\
\hline $\begin{array}{l}2 \\
7 \\
3 \\
5 \\
9 \\
4 \\
2 \\
2 \\
1 \\
2 \\
0 \\
1\end{array}$ & $\begin{array}{r}-4 \\
-3 \\
-2 \\
-1 \\
0 \\
1 \\
2 \\
3 \\
4 \\
5 \\
6 \\
7\end{array}$ & $\begin{array}{r}-8 \\
-21 \\
-6 \\
-5 \\
0 \\
4 \\
4 \\
6 \\
4 \\
10 \\
0 \\
7\end{array}$ & $\begin{array}{r}32 \\
63 \\
12 \\
5 \\
0 \\
4 \\
8 \\
18 \\
16 \\
50 \\
0 \\
49\end{array}$ \\
\hline $\begin{array}{c}38 \\
\text { Means } \ldots\end{array}$ & $\ldots$ & $\begin{array}{l}-5 \\
-\cdot 13\end{array}$ & $\begin{array}{l}257 \\
6.763\end{array}$ \\
\hline
\end{tabular}

$$
\sigma_{1}^{2}=6 \cdot 763-(\cdot 13)^{2}=6 \cdot 746 . \quad \sigma_{1}=2 \cdot 6 .
$$

(b) For $y$.

\begin{tabular}{|c|c|c|c|}
\hline Sum & Factor & 1st product & 2 nd product \\
\hline $\begin{array}{r}8 \\
11 \\
9 \\
4 \\
2 \\
1 \\
1 \\
2\end{array}$ & $\begin{array}{r}-2 \\
-1 \\
0 \\
1 \\
2 \\
3 \\
4 \\
5\end{array}$ & $\begin{array}{r}-16 \\
-11 \\
0 \\
4 \\
4 \\
3 \\
4 \\
10\end{array}$ & $\begin{array}{r}32 \\
11 \\
0 \\
4 \\
8 \\
9 \\
16 \\
50\end{array}$ \\
\hline $\begin{array}{c}38 \\
\text { Means }\end{array}$ & $\ldots \quad \ldots$ & $\begin{array}{l}-2 \\
-.05\end{array}$ & $\begin{array}{l}130 \\
3 \cdot 43\end{array}$ \\
\hline
\end{tabular}

These tables yield almost exactly the same values of $\sigma_{1}$ and $\sigma_{2}$ as were derived from the ungrouped material of observation.

Thus

$$
\sigma_{1}=2 \cdot 6 \text { units }=5 \cdot 2 \text { hours, }
$$

$$
\sigma_{2}=1.85 \text { units }=1.85 \times 30 \text { hours }=55.5 \text { hours. }
$$


In order to complete the calculation of $r$, we must calculate the product-moment $\Sigma x y$. The factors corresponding to $x y$ are the small figures given in the lower left-hand corners of the compartments of the table. In this particular example the sum of all the moments from any one quadrant can easily be evaluated. The result may be represented thus :

$$
\begin{array}{r|l}
+63 & -2 \\
\hline 0 & +98
\end{array} \quad \text { Total } 159 .
$$

'The product-moment thus obtained is not about the means of the two variables, and must be corrected.

$$
\begin{gathered}
r \sigma_{1} \sigma_{2}+\cdot 13 \times \cdot 05=\frac{159}{38}=4 \cdot 19, \\
r \sigma_{1} \sigma_{2}=4 \cdot 183, \\
\therefore r=\frac{4 \cdot 183}{2 \cdot 6 \times 1 \cdot 85}=870 .
\end{gathered}
$$

Thus the value of $r$ yielded by this method is 870 as compared with $\cdot 881$ obtained by working with the original ungrouped material.

When the number of observations represented in the contingency table is large, the product-moment cannot be obtained quite as simply as was done above. It may then be advisable to write in one column all the factors which are effective, writing the corresponding frequencies, with due regard to sign, in a second column. The remainder of the calculation is then easy.

\section{The Correlation Ratio.}

If the curve of regression be not linear, $r$ cannot be regarded as a satisfactory measure of the amount of correlation between the two characters considered. We then have to find a method which will decide whether the observations are clustered closely about the curve of regression, or are widely scattered. The obvious method is to evaluate the S.D. of each array.

Let $s_{a x}$ be the S.D. of any $x$-array. Then once the quantities 
$s_{a x}$ have been evaluated for all the arrays, we can tell accurately how closely the individual poines in the scatter diagram are clustered about the curve of regression. A curve representing $\frac{s_{a x}}{\sigma_{2}}$ is called a scedlustic curve. The mean ordinate of such a curve is a measure of the closeness with which a given value of $x$ yields a definite value of $y$. In praetice, however, it is more eonvenient to evaluate the S.D. of the weighted S.D.'s of the separate $x$-arrays. Let this s D. be $\sigma_{a x}$.

$$
\text { Then } \quad \sigma_{a x}^{2}=\frac{\sum n s_{a x}^{2}}{N} .
$$

It is found that $\sigma_{a x}^{2}$ can be calculated without going through the process of evaluating $s_{a x}$ for each $x$-array.

Let $M_{y}$ denote the mean of all $y$ 's, $m_{y}$ the mean of any $x$-array* whose S.D. is $s_{a x}$. Summing for one array, we find

$$
\begin{aligned}
\Sigma\left(y-M_{y}\right)^{2} & =\Sigma\left(y-m_{y}\right)^{2}+n\left(M_{y}-m_{y}\right)^{2} \\
& =n s_{a x}^{2}+n\left(M_{y}-m_{y}\right)^{2} .
\end{aligned}
$$

Summing for all arrays, we find

$$
N \sigma_{2}^{2}=N \sigma_{a x}^{2}+N \sigma_{m y}^{2} \text {, }
$$

where $\sigma_{m y}$ is the S.D. of the weighted means of the separate $x$-arrays.

$$
\therefore \quad \sigma_{a x}^{2}=\sigma_{2}{ }^{2}-\sigma^{2}{ }_{m y}=\sigma_{2}{ }^{2}\left(1-\eta_{y x}^{2}\right) \text { say, }
$$

where

$$
\eta_{y x}=\frac{\sigma_{m y}}{\sigma_{2}} \text {. }
$$

The quantity $\eta_{y x}$ thus defined is called the correlation ratio of $y$ on $x$. There will naturally be a second correlation ratio, $\eta_{x y}$, which is the correlation ratio of $x$ on $y$. Its value is $\frac{\sigma_{m x}}{\sigma_{1}}$.

The mean square of the distance between the curve of regression and the line of regression $y=r \frac{\sigma_{2}}{\sigma_{1}} x$ is

$$
\begin{aligned}
\frac{1}{N} \Sigma n_{y}\left(\bar{y}_{x}-r \frac{\sigma_{2}}{\sigma_{1}} x\right)^{2} & =\frac{\Sigma n_{y} \bar{y}_{x}{ }^{2}}{N}+\frac{r^{2} \sigma_{2}{ }^{2}}{\sigma_{1}{ }^{2}} \frac{\sum n_{y} x^{2}}{N}-2 r \frac{\sigma_{2}}{\sigma_{1}} \frac{\Sigma n_{y} x \bar{y}_{x}}{N} \\
& =\sigma_{m y}^{2}+\frac{r^{2} \sigma_{2}{ }^{2}}{\sigma_{1}{ }^{2}} \sigma_{1}{ }^{2}-2 r \frac{\sigma_{2}}{\sigma_{1}} r \sigma_{1} \sigma_{2} \\
& =\sigma_{m y}^{2}-r^{2} \sigma_{2}{ }^{2} \\
& =\sigma_{2}{ }^{2}\left(\eta^{2}{ }_{y x}-r^{2}\right) .
\end{aligned}
$$

* An $x$-array is an array of $y$ 's.

B. 0 . 
Thus $\eta^{2}-r^{2}$ is a measure of the deviation from linearity of the curve of regression. In accurate work it is advisable to calculate $\eta$ as well as $r$, since $\eta$ is a better measure of causal relation than $r$, and $\eta^{2}-r^{2}$ affords a measure of the linearity of the regression. It should be noted that $\eta$ is always greater than $r$, except when the regression is accurately linear, and in this case $\eta=r$. And conversely, if $\eta=r$, within the limits of random sampling, the regression is linear.

It is clear from the equation

$$
1-\eta_{y x}^{2}=\frac{\sigma_{a x}^{2}}{\sigma_{2}^{2}}
$$

that $|\eta|$ must lie between 0 and 1 , and since $\eta$ is the ratio of two S.D.'s it must be positive. The correlation ratio therefore lies between 0 and $\mathbf{1}$ in all cases. When the correlation is complete $\eta$ is unity, and when there is no correlation between the variables, $\eta$ is zero. When there is a considerable amount of correlation between the variables, $\eta$ is large, and when the variables are only slightly correlated $\eta$ is small. But the correlation ratio only affords a satisfactory test when the number of observations is sufficiently great to permit of the formation of a grouped contingency table.

\section{Probable Errors.}

As all the quantities $\sigma, r, \eta$, etc., mentioned above are in general calculated from a sample of the total population which bears the characters considered, it is necessary to consider the probable errors of all these quantities. Remembering that

we may write

$$
\sigma=\mu \sqrt{\frac{n-1}{n}}
$$

$$
\begin{aligned}
& \text { P.E. of mean }=\cdot 6745 \frac{\mu}{\sqrt{n}}=\cdot 6745 \frac{\sigma}{\sqrt{n}} \text { (approximately), } \\
& \text { P.E. of } \sigma=\cdot 6745 \frac{\sigma}{\sqrt{2(n-1)}}=6745 \frac{\sigma}{\sqrt{2 n}} \text { (approximately). }
\end{aligned}
$$

The P.E. of $r$ may be taken to be

$$
6745 \frac{1-r^{2}}{\sqrt{n}}
$$


in cases where the frequency distribution is approximately normal, and $n$ is large.

When the regression is linear, or nearly linear, the P.E. of $\eta$ is

$$
6745 \frac{1-\eta^{2}}{\sqrt{n}} .
$$

These formulae are based upon certain definite assumptions concerning the relations between the variables considered. It can be shown that if the variations of $x$ and $y$ from their mean values are due to a number of independent sources of error, some of which are common to both, while each of the elementary errors follows a normal law of distribution, then the number of cases in which $x$ lies between $x$ and $x+d x$, while $y$ lies between $y$ and $y+d y$, will be $f(x, y) d x d y$, where

$$
f(x, y)=A e^{-\frac{1}{2\left(1-r^{2}\right)}\left(\frac{x^{2}}{\sigma_{1}^{2}}+\frac{y^{2}}{\sigma_{2}^{2}}-\frac{2 x y r}{\sigma_{1} \sigma_{2}}\right)} .
$$

It will be noted that this law of distribution requires that both $x$ and $y$, when considered independently, should follow a normal law of distribution.

The P.E.'s of $r$ and $\eta$ given above are calculated by the use of the formula here given for $f(x, y)$. The methods of correlation are frequently applied to variables which do not follow a normal law of variation, and the probable errors of $r$ and $\eta$ are still calculated by the use of the formulae given above. It must be remembered that in such cases the formulae cannot yield more than a rough approximation to the values of the probable errors.

\section{To calculate $\eta$ for the material of the table on} page 151.

The following is a rough estimate of the value of $\eta$. It is necessary to evaluate $m_{y}$ for each $x$-array. For the purpose of a rough calculation, it is sufficient to assume the mean value of $y$ for the observations in any one compartment to be the central value for that compartment. The value of $m_{y}$ is then easily evaluated. The mean value of all the $y$ 's has already been shown to be $82 \cdot 7$. This is the quantity called $M_{y}$. 


\begin{tabular}{|c|c|c|c|c|c|}
\hline$x$ & $n$ & $m_{y}$ & $\boldsymbol{M}_{y}-m_{y}$ & $\left(M_{y}-m_{y}\right)^{2}$ & $n\left(M_{y}-m_{y}\right)^{2}$ \\
\hline $\begin{array}{r}2-4 \\
4-6 \\
6-8 \\
8-10 \\
10-12 \\
12-14 \\
14-16 \\
16-18 \\
18-20 \\
20-22 \\
22-24 \\
24-26\end{array}$ & $\begin{array}{l}2 \\
7 \\
3 \\
5 \\
9 \\
4 \\
2 \\
2 \\
1 \\
2 \\
1\end{array}$ & $\begin{array}{r}25 \cdot 0 \\
29 \cdot 3 \\
55 \cdot 0 \\
73 \cdot 0 \\
81 \cdot 7 \\
70 \cdot 0 \\
130 \cdot 0 \\
175 \cdot 0 \\
145 \cdot 0 \\
220 \cdot 0 \\
- \\
175 \cdot 0\end{array}$ & $\begin{array}{r}-57 \cdot 7 \\
-53 \cdot 4 \\
-27 \cdot 7 \\
-\quad 9 \cdot 7 \\
-\quad 1 \cdot 0 \\
-12 \cdot 7 \\
47 \cdot 3 \\
92 \cdot 3 \\
62 \cdot 3 \\
137 \cdot 3 \\
-\overline{92} \cdot 3\end{array}$ & $\begin{array}{r}3329 \\
2851 \\
765 \\
94 \\
1 \\
161 \\
2237 \\
8519 \\
3581 \\
18851 \\
- \\
8519\end{array}$ & $\begin{array}{r}6658 \\
19957 \\
2295 \\
470 \\
19 \\
644 \\
4474 \\
17038 \\
3581 \\
37702 \\
\\
8519\end{array}$ \\
\hline & 38 & & & & 101357 \\
\hline \multicolumn{6}{|c|}{$\frac{101357}{38}=2667$} \\
\hline
\end{tabular}

The value of $r$ was found to be 881 .

76. Spearman's Formulae. The IMethod of Ranks*.

In the method of ranks the actual measurements are replaced by the numbers representing the ranks of measurements when they are arranged in order of magnitude. Spearman applied the method to the consideration of the correlation of psychical performances in individuals. If $N$ individuals be considered, and $\nu_{1}, \nu_{2}$ be the orders of merit of any one individual in the two series of measurements, the product-moment reduces to

$$
1-\frac{6 \Sigma\left(\nu_{1}-\nu_{2}\right)^{2}}{N\left(N^{2}-1\right)}=\rho .
$$

Spearman also suggested a simpler formula, for the productmoment,

$$
\frac{\sum d}{1-\frac{1}{6}\left(N^{2}-1\right)}=R
$$

where $\Sigma d$ is the sum of gains, in rank (or the sum of positive differences) of the second series on the first.

The method of deduction of these formulae assumes that the

* Amer. Jour. Psych., Vol. xv, 1904; Brit. Jour. Psych., Vol. Ir, part 1. 
frequency distribution can be represented by a rectangle. Pearson* has shown that if the frequency distribution is Gaussian, the quantities $\rho$ and $R$ are connected with $r$ by the relations

$$
r=2 \sin \left(\frac{\pi}{6} \rho\right), \quad r=2 \cos \frac{\pi}{3}(1-R)-1 .
$$

When the frequency distribution is not Gaussian, it is not clear how $r$ is to be deduced from $\rho$ or $R$. For this reason, the above formulae are not to be recommended for use in accurate work. A further disadvantage is that the method does not yield the values of the standard deviations. The formulae may be useful however in rough work involving only a small number of observations.

In order to compare the results yielded by Spearman's formulae with the result deduced from the product-moment formula, we shall apply these formulae to the example of page 194. 'The stars are arranged in order of magnitude of orbital period in the table of page 194. The positions of these stars in a table arranged in order of magnitude of eclipseduration are given in the second column below.

\begin{tabular}{|c|c|c|c|c|c|c|c|}
\hline$\nu_{1}$ & $\nu_{2}$ & $d$ & $d^{2}$ & $\nu_{1}$ & $\nu_{2}$ & $d$ & $d^{2}$ \\
\hline 1 & 1 & 0 & 0 & 20 & 13 & 7 & 49 \\
\hline 2 & 2 & 0 & 0 & 21 & 24 & -3 & 9 \\
\hline 3 & 8 & -5 & 25 & .22 & 27 & -5 & 25 \\
\hline 4 & 4 & 0 & 0 & 23 & 25 & -2 & 4 \\
\hline 5 & 4 & 1 & 1 & 24 & 30 & -6 & 36 \\
\hline 6 & 4 & 2 & 4 & 25 & 23 & 2 & 4 \\
\hline 7 & 9 & -2 & 4 & 26 & 17 & 9 & 81 \\
\hline 8 & 3 & 5 & 25 & 27 & 18 & 9 & 81 \\
\hline 9 & 4 & 5 & 25 & 28 & 16 & 12 & 144 \\
\hline 10 & 10 & 0 & 0 & 29 & 21 & 8 & 64 \\
\hline 11 & 10 & 1 & 1 & 30 & 25 & 5 & 25 \\
\hline 12 & 27 & -15 & 225 & 31 & 31 & 0 & 0 \\
\hline 13 & 22 & -9 & 81 & 32 & 33 & -1 & 1 \\
\hline 14 & 18 & -4 & 16 & 33 & 32 & 1 & 1 \\
\hline 15 & 13 & 2 & 4 & 34 & 35 & -1 & 1 \\
\hline 16 & 13 & 3 & 9 & 35 & 38 & -3 & 9 \\
\hline 17 & 12 & 5 & 25 & 36 & 36 & 0 & 0 \\
\hline 18 & 27 & -9 & 81 & 37 & 37 & 0 & 0 \\
\hline 19 & 18 & 1 & 1 & 38 & 34 & 4 & 16 \\
\hline
\end{tabular}

* Drapers' Company Research Memoirs, Biometric Series IV. 


$$
\begin{gathered}
\quad \Sigma d=17 . \quad \Sigma d^{2}=1077 . \\
\therefore R=1-\frac{6 \cdot 17}{38^{2}-1}=1-\cdot 071=\cdot 929, \\
\rho=1-\frac{6.1077}{38\left(38^{2}-1\right)}=\cdot 882 .
\end{gathered}
$$

Using the formulae given above, we find

$$
\begin{aligned}
& r=2 \sin \left(\frac{\pi}{6} \times \cdot 882\right)=\cdot 891, \\
& r=2 \cos \frac{\pi}{3}(\cdot 071)-1=\cdot 994 .
\end{aligned}
$$

The value derived for $r$ by the use of the product-moment formula is $\cdot 881$.

\section{The Method of Contingency.}

The methods discussed above are only applicable in cases where the characters considered are capable of quantitative measurement. Such characters would be length, time, stellar magnitude, wages, etc. When the grouping in the contingency table is purely classificatory, the different classes bear no quantitative relation to each other. Thus if stars be grouped according to a colour scale, the scale cannot be regarded as an arithmetical scale. Except for this difference in the nature of the scale, tables which represent the distribution of two characters, of which one or both may be purely qualitative, are of precisely the same form as the contingency table given on page 151. The following is an example of such a contingency table*, representing the distribution of 1360 stars according to spectral type and colour. The groupings $a, b, c, d, e, f$ are according to a scale of colour, where

stars of class $a$ are white,

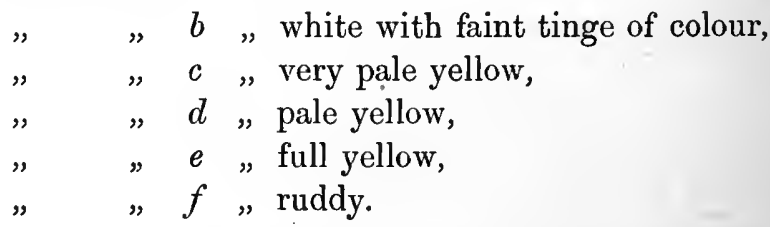

* W. S. Franks, "On the Relation between Star Colours and Spectra,"Monthly Notices, R.A.S., Vol. Lxvir, p. 541, Table IV. 


\begin{tabular}{|c|c|c|c|c|c|c|c|}
\hline Spectral Type & $a$ & b) & $c$ & $d$ & $e$ & $f$ & 'lotal \\
\hline Helmon Stars & $\begin{array}{c}125 \\
(61 \cdot 38)\end{array}$ & $\begin{array}{c}146 \\
(103 \cdot 05)\end{array}$ & $\begin{array}{c}8 \\
(4+37)\end{array}$ & $\begin{array}{c}3 \\
(37 \cdot 74)\end{array}$ & $\begin{array}{c}0 \\
(33 \cdot 18)\end{array}$ & $\begin{array}{c}0 \\
(2 \cdot 28)\end{array}$ & 282 \\
\hline Hydrogen Stars & $\begin{array}{c}168 \\
(82 \cdot 05)\end{array}$ & $\begin{array}{c}195 \\
(137 \cdot 77)\end{array}$ & $\begin{array}{c}14 \\
(59 \cdot 32)\end{array}$ & $\begin{array}{c}0 \\
(50 \cdot 45)\end{array}$ & $\begin{array}{c}0 \\
(44 \cdot 35)\end{array}$ & $\begin{array}{c}0 \\
(3 \cdot 05)\end{array}$ & 377 \\
\hline a Carinae Type & $\begin{array}{c}3 \\
(29 \cdot 82)\end{array}$ & $\begin{array}{c}97 \\
(50 \cdot 07)\end{array}$ & $\begin{array}{c}23 \\
(21 \cdot 56)\end{array}$ & $\begin{array}{c}8 \\
(18 \cdot 33)\end{array}$ & $\begin{array}{c}6 \\
(16 \cdot 12)\end{array}$ & $\begin{array}{c}0 \\
(1 \cdot 11)\end{array}$ & 137 \\
\hline Solar Stars & $\begin{array}{c}0 \\
(39 \cdot 18)\end{array}$ & $\begin{array}{c}41 \\
(65 \cdot 78)\end{array}$ & $\begin{array}{c}77 \\
(28 \cdot 32)\end{array}$ & $\begin{array}{c}3: 3 \\
(24 \cdot 09)\end{array}$ & $\begin{array}{c}29 \\
(21 \cdot 18)\end{array}$ & $\begin{array}{c}0 \\
(1 \cdot 46)\end{array}$ & 180 \\
\hline Arcturus Type & $\begin{array}{c}0 \\
(52 \cdot 45)\end{array}$ & $\begin{array}{c}15 \\
\left(88^{\circ} \cdot 07\right)\end{array}$ & $\begin{array}{c}86 \\
(37 \cdot 92)\end{array}$ & $\begin{array}{c}77 \\
(32 \cdot 25)\end{array}$ & $\begin{array}{c}63 \\
(28 \cdot 35)\end{array}$ & $\begin{array}{c}0 \\
(1 \cdot 95)\end{array}$ & 241 \\
\hline Aldebaran Type & $\begin{array}{c}0 \\
(16 \cdot 32)\end{array}$ & $\begin{array}{c}0 \\
(27 \cdot 41)\end{array}$ & $\begin{array}{c}4 \\
(11 \cdot 80\end{array}$ & $\begin{array}{c}22 \\
\left(10^{\circ} 04\right)\end{array}$ & $\begin{array}{c}43 \\
(8 \cdot 82)\end{array}$ & $\begin{array}{l}6 \\
(\cdot 61)\end{array}$ & 75 \\
\hline Betelgeuse & $\begin{array}{c}0 \\
(14 \cdot 80)\end{array}$ & $(24 \cdot \stackrel{3}{85})$ & $\begin{array}{c}2 \\
(10 \cdot 70)\end{array}$ & $\begin{array}{c}39 \\
(9 \cdot 10)\end{array}$ & $\begin{array}{c}19 \\
\left(8^{\circ} 00\right)\end{array}$ & $\stackrel{5}{\cdot 55})$ & 68 \\
\hline Total & 296 & 497 & 214 & 182 & 160 & 11 & 1360 \\
\hline
\end{tabular}

Leaving out of consideration for the moment the figures in brackets in each compartment, we have a contingency table resembling that of page 151. Even a casual examination of this table shows that there is a tendency for stars low down in the scale of spectra to have colours which are nearer to red than those of stars higher in the scale of spectra ; i.e. there is some degree of correlation between colour and spectral type of stars.

The last row in the table gives the distribution according to colour, and the last column the distribution according to spectral type, of all the stars considered. If spectral type and colour were independent factors, the number of stars in any compartment of the table could be evaluated from the totals in the last row and the last column; e.g. if colour were independent of spectral type, the number of stars of colour $c$ and of solar type would be

$$
\frac{214}{1360} \times 180 \text {, or } 28.32 \text {. }
$$

The numbers which should occur in the other compartments can be evaluated in the same way. These numbers are entered in the compartments of the table, in brackets. These bracketed 
figures give the distribution to be expected if there were no correlation between spectral type and colour. The differences between the two sets of figures are due to correlation between the two characters.

It is customary to calculate the amount of correlation in the following way:

Form the difference between the bracketed and unbracketed figures in each compartment; square this difference and divide the result by the corresponding bracketed figure. Then add together all the numbers obtained in this manner, and divide the sum by the total number of stars. This result is called the total mean square contingency, and is denoted by $\phi^{2}$.

For the example shown in the above table,

$$
1360 \phi^{2}=\frac{(125-61 \cdot 38)^{2}}{61 \cdot 38}+\frac{(146-103 \cdot 05)^{2}}{103 \cdot 05}+\text { etc., }
$$

there being 42 terms in all;

$$
\phi^{2}=1 \cdot 021
$$

The function $c$ defined by

$$
c=\sqrt{\frac{\phi^{2}}{1+\phi^{2}}}
$$

is called the coeffcient of mean square contingency. The quantity $c$ is of the same nature as the coefficient of correlation $r$. When the frequency distribution is normal, it can be shown that $c$ calculated as above is equal to $r$. It is clear from its definition that $c$ must lie between -1 and +1 . When there is no correlation between the two characters considered,

$$
\phi=0 \text { and } c=0 .
$$

Small values of $\phi$ and $c$ indicate a small amount of correlation.

For the table given above

$$
c=\cdot 71,
$$

a value which may be taken to indicate a close relationship between spectral type and colour.

For a full treatment of the subject of contingency the reader is referred to the original memoir of Prof. Karl Pearson, "On the Theory of Contingency and its Relation to Association and Normal Correlation," Drapers' Company Research Memoirs, Biometric Series I, 1904, Dulau and Co. 
Example. Evaluate the s.D. of a linear function of a number of correlated variables.

$$
\text { Let } \quad F=a x+b y+c z+\ldots
$$

be the function.

Then $\quad r^{* 2}=a^{2} \cdot x^{2}+b^{2} y^{2}+c^{2} z^{2}+2 a b x y+2 a c x z+2 b c y z+\ldots$

It follows that

$$
\sigma_{z}^{2}=a^{2} \sigma_{x}^{2}+b^{2} \sigma_{y}{ }^{2}+c^{2} \sigma_{z}{ }^{2}+2 a b r_{x y} \sigma_{x} \sigma_{y}+2 a c r_{x z} \sigma_{x} \sigma_{z}+2 b c r_{y z} \sigma_{y} \sigma_{z}+\ldots
$$

A special case is $\quad \sigma_{x-y}^{2}=\sigma_{x}{ }^{2}+\sigma_{y}{ }^{2}-2 r_{x y} \sigma_{x} \sigma_{y}$.

This affords a method of evaluating $r_{x y}$ by evaluating

$$
\sigma_{x}, \quad \sigma_{y}, \quad \sigma_{x-y},
$$

\section{The Meaning of the Correlation Coefficient.}

The meaning which we shall assign to the correlation coefficient will to some extent depend upon the point of view from which we approach the question. Referring back to $\S 70$ we see that if the coefficient of correlation $r$ between two variables is known, the value of $x$ corresponding to any given value of $y$ can be given with a M.S.E. $s_{2}=\sigma_{2} \sqrt{1-r^{2}}, \sigma_{2}$ being the M.S.E. of all the $y$ 's. Our knowledge of the correlation $r$ thus enables us to reduce the M.S.E. of our estimate of the value of $y$ from $\sigma_{2}$ to $\sigma_{2} \sqrt{1-r^{2}}$. From this point of view it would appear preferable to measure correlation by $\sqrt{1-r^{2}}$ rather than by $r$.

It is customary, however, to regard $r$ as being in some way a measure of the number of common causes which underlie the variations of the two quantities considered. The following simple case considered by Kapteyn may help to make this point clear. Let the variations of $x$ and $y$ be due to a number $m+n$ of elementary causes, $m$ of these causes being common to both $x$ and $y$, while the remaining $n$ causes are independent. Then we may write

$$
\begin{aligned}
& x=A_{1} \alpha_{1}+A_{2} \alpha_{2}+\ldots+A_{m} \alpha_{n}+B_{1} \beta_{1}+B_{2} \beta_{2}+\ldots+B_{n} \beta_{n}, \\
& y=D_{1} \alpha_{1}+D_{2} \alpha_{2}+\ldots+D_{m} \alpha_{m}+C_{1} \gamma_{1}+C_{2} \gamma_{2}+\ldots+C_{n} \gamma_{n},
\end{aligned}
$$

where $\alpha_{1}, \alpha_{2}, \ldots, \beta_{1}, \beta_{2}, \ldots, \gamma_{1}, \gamma_{2}, \ldots$, are all independent of one another. We shall further simplify the problem by supposing that 
the M.S.E. introduced into $x$ or $y$ by any one of the independent elementary causes is unity. Then

$$
\begin{aligned}
& x=\sum_{1}^{m} \alpha_{s}+\sum_{1}^{n} \beta_{s}, \\
& y=\sum_{1}^{m} \alpha_{s}+\sum_{1}^{n} \gamma_{s},
\end{aligned}
$$

where the M.S.E. of each variable $\alpha, \beta$, or $\gamma$, is unity. Then if $\epsilon_{x}, \epsilon_{y}$ be the M.S.E. of $x$ and $y$ respectively,

$$
\begin{gathered}
\epsilon_{x}{ }^{2}=m+n, \quad \epsilon_{y}{ }^{2}=m+n, \\
x+y=2 \sum_{1}^{m} \alpha_{s}+\sum_{1}^{n} \beta_{s}+\sum_{1}^{n} \gamma_{s}, \\
(x+y)^{2}=4 \sum_{1}^{m} \alpha_{s}{ }^{2}+\sum_{1}^{n} \beta_{s}{ }^{2}+\sum_{1}^{n} \gamma_{s}{ }^{2}+4 \sum \alpha_{s} \alpha_{t} \\
+2 \Sigma \beta_{s} \beta_{t}+2 \Sigma \gamma_{s} \gamma_{t}+4 \Sigma \alpha_{s} \beta_{t}+4 \Sigma \alpha_{s} \gamma_{t} \\
+2 \Sigma \beta_{s} \gamma_{t} .
\end{gathered}
$$

If $\epsilon_{x+y}$ be the M.S. E. of $x+y$, then the mean value of the R.H.S. of the above equation is equal to $\epsilon_{x+y}^{2}$;

$$
\therefore \quad \epsilon_{x+y}^{2}=4 \sum_{1}^{m} \bar{a}_{8}^{2}+\sum_{1}^{n} \bar{\beta}_{8}{ }^{2}+\sum_{1}^{n} \bar{\gamma}_{8}{ }^{2}=4 m+n+n,
$$

the product terms all vanishing.

The last equation may be written

$$
\begin{aligned}
\epsilon_{x+y}^{2} & =4 m+2 n \\
& =\epsilon_{x}{ }^{2}+\epsilon_{y}{ }^{2}+2 r \epsilon_{x} \epsilon_{y}{ }^{*} \\
& =2 m+2 n+2 r(m+n) ; \\
\therefore & m=r(m+n), \\
r & =\frac{m}{m+n} .
\end{aligned}
$$

Thus in this particular case $r$ measures the proportion of elementary causes of variation which the two variables have in common.

* Vide Example, page 169. 


\section{CHAPTER XI}

HARMONIC ANALYSIS FROM THE STANDPOINT OF LEAST SQUARES

\section{To find the Amplitude of a Single Periodic Term of Known Period.}

For the sake of definiteness we shall consider the problem of finding the amplitude of the annual period in temperature records, from the mean monthly temperature. Let

$$
l_{0}, l_{1}, l_{2}, \ldots, l_{11}
$$

be the mean temperatures for each of twelve months, starting with January. Let the time be measured from the middle of January, and let the length of the month (assumed constant) be $\alpha$. Then the temperatures

$$
l_{0}, l_{1}, l_{2}, \ldots, l_{11}
$$

are associated with the times

$$
0, \alpha, 2 \alpha, \ldots, 11 \alpha .
$$

If $a$ be the required amplitude, we can write

$$
l=A_{0}+a \cos (\theta-\phi),
$$

where $l$ takes the value $l_{r}$ when

$$
\theta=r \frac{2 \pi}{12} \text {. }
$$

The variable $\theta$ passes through the range 0 to $2 \pi$, while the time passes through the range 0 to $12 \alpha$. Expanding the trigonometric term, we find

$$
l=A_{0}+A_{1} \cos \theta+B_{1} \sin \theta
$$

where $\quad A_{1}=a \cos \phi, \quad B_{1}=a \sin \phi, \quad a=\left(A_{1}^{2}+B_{1}\right)^{\frac{1}{2}}$. 
Giving $\theta$ the values $0, \alpha, 2 \alpha, \ldots, 11 \alpha$, in turn, we obtain 12 observational equations to be solved by the method of least squares. The normal equations, formed by the usual method, are

$$
\begin{aligned}
& \sum_{r=0}^{r=11}\left\{l_{r}-A_{0}-A_{1} \cos \theta_{r}-B_{1} \sin \theta_{r}\right\}=0, \\
& \sum_{r=0}^{r=11}\left\{l_{r}-A_{0}-A_{1} \cos \theta_{r}-B_{1} \sin \theta_{r}\right\} \cos \theta_{r}=0, \\
& \sum_{r=0}^{r=11}\left\{l_{r}-A_{0}-A_{1} \cos \theta_{r}-B_{1} \sin \theta_{r}\right\} \sin \theta_{r}=0 .
\end{aligned}
$$

Now it can be easily shown that*

$$
\begin{aligned}
& \Sigma \cos \theta_{r}=\Sigma \sin \theta_{r}=\Sigma \cos \theta_{r} \sin \theta_{r}=0, \\
& \Sigma \cos ^{2} \theta_{r}=\frac{1}{2} \Sigma\left(1+\cos 2 \theta_{r}\right)=6, \\
& \Sigma \sin ^{2} \theta_{r}=\frac{1}{2} \Sigma\left(1-\cos 2 \theta_{r}\right)=6 .
\end{aligned}
$$

* $\quad\{\cos a+\cos (\alpha+\beta)+\cos (\alpha+2 \beta)+\ldots+\cos (\alpha+\overline{n-1} \beta)\} \times 2 \sin \frac{1}{2} \beta$

$$
\begin{aligned}
&=\left\{\sin \left(\alpha+\frac{1}{2} \beta\right)-\sin \left(\alpha-\frac{1}{2} \beta\right)\right\}\left\{\sin \left(\alpha+\frac{3}{2} \beta\right)-\sin \left(\alpha+\frac{1}{2} \beta\right)\right\} \\
&+\left\{\sin \left(\alpha+\frac{5}{2} \beta\right)-\sin \left(\alpha+\frac{3}{2} \beta\right)\right\}+\ldots \\
&+\left\{\sin \left(a+\frac{2 n-1}{2} \beta\right)-\sin \left(a+\frac{2 n-3}{2} \beta\right)\right\} \\
&=\sin \left(\alpha+\frac{2 n-1}{2} \beta\right)-\sin \left(\alpha-\frac{1}{2} \beta\right) \\
&=2 \cos \left(\alpha+\frac{n-1}{2} \beta\right) \sin \frac{n \beta}{2} . \\
& \therefore \cos \alpha+\cos (\alpha+\beta)+\cos (\alpha+2 \beta)+\ldots \\
& \quad+\cos (\alpha+\overline{n-1} \beta)=\cos \left(\alpha+\frac{n-1}{2} \beta\right) \frac{\sin \frac{n \beta}{2}}{\sin \frac{\beta}{2}} .
\end{aligned}
$$

And similarly

$$
\begin{aligned}
& \sin \alpha+\sin (\alpha+\beta)+\sin (\alpha+2 \beta)+\ldots \\
& \quad+\sin (\alpha+\overline{n-1} \beta)=\sin \left(a+\frac{n-1}{2} \beta\right) \frac{\sin \frac{n \beta}{2}}{\sin \frac{\beta}{2}} .
\end{aligned}
$$

In the series $\Sigma \cos \theta_{r}, \Sigma \sin \theta_{r}, n=12, \quad \beta=\frac{2 \pi}{12}, \frac{n \beta}{2}=\pi$, and the factor $\sin \frac{n \beta}{2}$ vanishes.

In the series $\Sigma \sin 2 \theta_{r}, \Sigma \cos 2 \theta_{r}, \quad \beta=\frac{2 \pi}{12}, \frac{n \beta}{2}=2 \pi$, and the factor $\sin \frac{n \beta}{2}$ again vanishes. 
Substituting these values, we find*

$$
\left.\begin{array}{l}
A_{0}=\frac{1}{12} \sum_{r=0}^{r=11} l_{r} \\
A_{1}=\frac{1}{6} \sum_{r=0}^{r=11} l_{r} \cos \theta_{r} \\
B_{1}=\frac{1}{6} \sum_{r=0}^{r=11} l_{r} \sin \theta_{r}
\end{array}\right\}
$$

Equations (2) are the normal equations for determining the values of the constants $A_{0}, A_{1} ; B_{1}$. The evaluation can easily be carried out as follows: Write in a column the values of $l$, in a second column the values of $\cos \theta$, in a third the values of $\sin \theta$. Multiply the second and third columns by the first, writing the results in the fourth and fifth columns. From the sums of the first, fourth, and fifth columns, we can easily deduce the values of the constants. The headings of the different columns in the table will be

$$
l, \quad \cos \theta, \sin \theta, \quad l \cos \theta, l \sin \theta .
$$

This method, although it leads to the values of the constants, is not the shortest. On page 183 will be found a form of calculation which will shorten the work when there are 12 readings.

Equations (2) above can be immediately generalised for the case of $n$ equidistant readings,

$$
\left.\begin{array}{l}
n A_{0}=\Sigma l \\
\frac{n}{2} A_{1}=\Sigma l \cos \theta \\
\frac{n}{2} B_{1}=\Sigma l \sin \theta \\
n \pi-\frac{26 c}{\frac{\pi}{2}}
\end{array}\right\}
$$

80. General case involving more than one Period.

Let $l_{0}, l_{1}, l_{2}, \ldots, l_{n-1}$ be $n$ observed values, which are associated with equidistant values of some argument (say time). To fix ideas let us suppose the measurements $l_{0}, l_{1}$, etc., to be 
made at times $t_{0}, t_{0}+\alpha, t_{0}+2 \alpha, \ldots, t_{0}+\overline{n-1} \alpha$. Then it will be possible to represent the values $l_{0}, l_{1}$, etc., by means of a trigonometric series, thus,

$$
\left.\begin{array}{rl}
l=A_{0} & +A_{1} \cos \theta+A_{2} \cos 2 \theta+\ldots \\
& +B_{1} \sin \theta+B_{2} \sin 2 \theta+\ldots
\end{array}\right\}
$$

where $l$ takes the value $l_{r}$ when $\theta=r \frac{2 \pi}{n}$, and $r$ can take all positive integral values from 0 to $n-1$.

If in equation (4) we include $n$ constants, then by giving $r$ the successive values $0,1,2, \ldots, n-1$, we obtain $n$ equations, whose solution yields the values of the $n$ constants. We shall then have obtained an accurate representation of all $n$ observations by means of the series of trigonometric terms (4). In practice, however, this accurate solution is seldom required, and the problem is rather that of representing a series of $n$ observations, with fair accuracy, by means of a trigonometric series containing fewer than $n$ constants.

Such series as are represented in (4) are called Fourier series. It should be noted that the number of sine terms is generally taken to be either equal to, or one less than, the number of cosine terms. The reason for this will appear in the sequel.

81. We shall now consider the problem as it arises in practice, i.e. how we shall best represent a series of $n$ observations by means of a series

$$
\left.\begin{array}{rl}
l=A_{0} & +A_{1} \cos \theta+A_{2} \cos 2 \theta+\ldots+A_{m} \cos m \theta \\
& +B_{1} \sin \theta+B_{2} \sin 2 \theta+\ldots+B_{m} \sin m \theta
\end{array}\right\} \ldots(5),
$$

or

where

$$
n>2 m+1 \text {. }
$$

We have $n$ observational equations, which we shall solve by the methods of least squares. The normal equations are 


$$
\begin{aligned}
\sum_{r=0}^{r=n-1} l_{r}-n A_{0}- & \sum_{r=0}^{r=n-1} \sum_{i=1}^{i=m} A_{i} \cos \frac{2 \pi r i}{n} \\
& \quad-\sum_{r=0}^{r=n-1} \sum_{i=1}^{i=m} B_{i} \sin \frac{2 \pi r i}{n}=0
\end{aligned}
$$

with $m$ equations of the form

$$
\begin{aligned}
\sum_{r=0}^{r=n-1} l_{r} \cos \frac{2 \pi r s}{n} & -\sum_{r=0}^{r=n-1} A_{0} \cos \frac{2 \pi r s}{n} \\
& -\sum_{r=0}^{r=n-1} \sum_{i=1}^{i=m} A_{i} \cos \frac{2 \pi r i}{n} \cos \frac{2 \pi r s}{n} \\
& -\sum_{r=0}^{r=n-1} \sum_{i=1}^{i=m} B_{i} \sin \frac{2 \pi r i}{n} \cos \frac{2 \pi r s}{n}=0 \\
s & =1,2, \ldots, m,
\end{aligned}
$$

where

and $m$ equations of the form

$$
\begin{aligned}
\sum_{r=0}^{r=n-1} l_{r} \sin \frac{2 \pi r s}{n} & -\sum_{r=0}^{r=n-1} A_{0} \sin \frac{2 \pi r s}{n} \\
& -\sum_{r=0}^{r=n-1} \sum_{i=1}^{i=m} A_{i} \cos \frac{2 \pi r i}{n} \sin \frac{2 \pi r s}{n} \\
& -\sum_{r=0}^{r=n-1} \sum_{i=1}^{i=m} B_{i} \sin \frac{2 \pi r i}{n} \sin \frac{2 \pi r s}{n}=0 \\
\text { re } & s=1,2, \ldots, m .
\end{aligned}
$$

where

The series $\Sigma \cos \frac{2 \pi r i}{n}, \Sigma \sin \frac{2 \pi r i}{n}$ are of the form of the series given in the footnote on page 172 , where $\beta=\frac{2 \pi i}{n}$, so that

$$
\sin \frac{n \beta}{2}=\sin \pi i=0 \text {. }
$$

Similarly, when $s$ is not equal to $i$,

$$
\begin{aligned}
\sum_{r=0}^{r=n-1} \cos \frac{2 \pi r i}{n} & \sin \frac{2 \pi r s}{n} \\
\quad=\frac{1}{2} & \sum_{r=0}^{r=n-1}\left\{\sin \frac{2 \pi r(s+i)}{n}+\sin \frac{2 \pi r(s-i)}{n}\right\}=0,
\end{aligned}
$$

since each of the series is zero*. In the same way it can be

* Compare with footnote, page 172. 
shown that

$$
\begin{aligned}
\sum_{r=0}^{r=n-1} \cos \frac{2 \pi r i}{n} \cos \begin{aligned}
2 \pi r s \\
n
\end{aligned} & =\sum_{r=0}^{r=n-1} \sin \frac{2 \pi r i}{n} \cos \frac{2 \pi r s}{n} \\
& =\sum_{r=0}^{r=n-1} \sin \frac{2 \pi r i}{n} \sin \frac{2 \pi r s}{n}=0
\end{aligned}
$$

when $s$ is not equal to $i$.

When $s=i$

$$
\begin{gathered}
\sum_{r=0}^{r=n-1} \cos ^{2} \frac{2 \pi r s}{n}=\frac{1}{2} \sum_{r=0}^{r=n-1}\left(1+\cos \frac{4 \pi r s}{n}\right)=\frac{n}{2}, \\
\sum_{r=0}^{r=n-1} \cos \frac{4 \pi r s}{n}=0 .
\end{gathered}
$$

since

Similarly

$$
\sum_{r=0}^{r=n-1} \sin ^{2} \frac{2 \pi r s}{n}=\frac{1}{2} \sum_{r=0}^{r=n-1}\left(1-\cos \frac{4 \pi r s}{n}\right)=\frac{n}{2} .
$$

Substituting these values for the trigonometric series which occur in the normal equations, we obtain the following simplified form of the normal equations:

where

$$
\begin{aligned}
& \sum_{r=0}^{r=n-1} l_{r}-n A_{0}=0 \\
& \sum_{r=0}^{r=n-1} l_{r} \cos \frac{2 \pi r s}{n}-\frac{n}{2} A_{s}=0 \\
& \sum_{r=0}^{r=n-1} l_{r} \sin \frac{2 \pi r s}{n}-\frac{n}{2} B_{s}=0
\end{aligned}
$$

$$
s=1,2, \ldots, m \text {. }
$$

$$
n>2 m+1 \text {. }
$$

Since the coefficients $A_{s}, B_{8}$ derived from equations (7) are obviously independent of one another, the work may, if necessary, be carried out by successive stages, until a sufficiently close approximation to the series of observations is obtained. It will be shown that the addition of a pair of extra terms to the series improves the accuracy of the representation of the observations by the series.

If we start with the assumption

$$
\left.\begin{array}{rl}
l=A_{0} & +A_{1} \cos \theta+A_{2} \cos 2 \theta \\
& +B_{1} \sin \theta+B_{2} \sin 2 \theta
\end{array}\right\}
$$


the coefficients $A_{0}, A_{1}, A_{2}, B_{1}, B_{3}$ may be evaluated by means of equations (7): If we should then require to carry the work a stage further, we might use the equation

$$
\left.\begin{array}{rl}
l=A_{0} & +A_{1} \cos \theta+A_{2} \cos 2 \theta+A_{3} \cos 3 \theta \\
& +B_{1} \sin \theta+B_{2} \sin 2 \theta+B_{3} \sin 3 \theta
\end{array}\right\}
$$

The least squares solution of equation $(b)$ is the same as that of equation (a) as far as the evaluation of $A_{0}, A_{1}, A_{2}, B_{1}, B_{2}$ is concerned, and the only fresh work involved is the evaluation of $A_{3}$ and $B_{3}$. These coefficients are obtained by the use of equations (7).

The property of independence of the coefficients leads to an important result. If we adopt equation $(b)$ above, $[v v]$ is a minimum when the coefficients in this equation are deduced from equations (7). Hence $[v v]$ calculated from equation $(b)$ is less than would be obtained by putting $A_{3}=0, B_{3}=0$; i.e. it is less than $[v v]$ calculated from equation $(a)$. It follows that each successive pair of terms added to the Fourier series means a gain in the accuracy with which the observations are represented by the series. As we go on adding terms to the Fourier series the residuals diminish continually, until, when the number of terms in the series becomes equal to the number of observations, the residuals all vanish, and the representation of the observations by the series is perfect.

\section{Evaluation of [vv].}

The residuals are given by the equations

$$
v_{r}+l_{r}=A_{0}+\sum_{i=1}^{i=m} A_{i} \cos \frac{2 \pi r i}{n}+\sum_{i=1}^{i=m} B_{i} \sin \frac{2 \pi r i}{n},
$$

where

$$
r=0,1,2, \ldots, n-1 \text {. }
$$

Multiplying each such equation by the corresponding $l_{r}$, and remembering that $[v v]=-[v l]$, we obtain the relation

$$
\begin{aligned}
{[v v]=[l l]-A_{0} \Sigma l_{r}-} & \sum_{r=0}^{r=n-1} \sum_{i=1}^{i=m} l_{r} A_{i} \cos \frac{2 \pi r i}{n} \\
& \quad-\sum_{r=0}^{r=n-1} \sum_{i=1}^{i=m} l_{r} B_{i} \sin \frac{2 \pi r i}{n} \ldots . .(8) .
\end{aligned}
$$

B. 0 . 
The coefficient of $-A_{i}$ in this equation is

$$
\sum_{r=0}^{r=n-1} l_{r} \cos \frac{2 \pi r i}{n},
$$

which by equations $(7)$ is $\frac{n}{2} A_{i}$; and the coefficient of $-B_{i}$ is

$$
\sum_{r=0}^{r=n-1} l_{r} \sin \frac{2 \pi r i}{n} \text { or } \frac{n}{2} B_{i} .
$$

Thus equation (8) may be written

$$
[v v]=[l l]-n A_{0}^{2}-\frac{n}{2} \sum_{i=1}^{i=w}\left(A_{i}^{2}+B_{i}^{2}\right)
$$

Equation (9) shows that the addition of each successive pair of terms to the Fourier series decreases [vv], while, as we have already seen, this in no way affects the preceding terms.

\section{Case where $\mathbf{n}$ terms are taken in the Fourier} Series.

If $n$ be an odd number, the greatest value of $m$ is given by

$$
n=2 m+1 \quad \text { or } \quad m=\frac{n}{2}-\frac{1}{2}=\frac{n-1}{2} \text {. }
$$

The Fourier series can thus be carried as far as the terms involving $\frac{n-1}{2} \theta$. The series then gives an exact representation of the $n$ observations, and all the coefficients can be deduced by the use of equations (7) above.

When $n$ is an even number, it is not possible to carry the sine terms as far as $B_{\frac{n}{2}}$, since equations (7) yield zero for the value of $B_{s}$ when $s=\frac{n}{2}$. The sine terms must therefore stop at

$$
B_{\frac{n}{2}-1} \sin \left(\frac{n}{2}-1\right) \theta
$$

Equations (6) yield a determinate value for $A_{\frac{n}{2}}$, and the cosine series can therefore be carried as far as $A_{\frac{n}{2}} \cos \frac{n}{2} \theta$. Returning 
to equations (6) and putting $s=\frac{n}{2}$, we find that the coefficient of $A_{\frac{n}{2}}$ is $\sum_{r=0}^{r=n-1} \cos ^{3} \pi r$ or $n$, and so $A_{\frac{n}{2}}$ is given by the equation

$$
n A_{\frac{n}{2}}=\Sigma\left(l_{r} \cos \pi r\right)=\Sigma(-1)^{r} l_{r}
$$

Note the factor $n$ which replaces the $\frac{n}{2}$ of equations (7).

A slight modification has to be made in equation (9). For, in equation (8) the coefficient of $A_{\frac{n}{2}}$ is $\Sigma\left(l_{r} \cos \pi r\right)$ or $n A_{\frac{n}{2}}$.

Equation (9) then reads

$$
[v v]=[l l]-n A_{0}{ }^{2}-n_{2}^{n} \sum_{i=1}^{i=\frac{n}{2}-1}\left(A_{i}{ }^{2}+B_{i}{ }^{2}\right)-n A_{\frac{n}{2}}{ }^{2} \ldots . .(11) .
$$

It should be noted that equations (10) and (11) are only required when it is desired to obtain an accurate representation of an even number of observations by a Fourier series containing as many terms as there are observations. In all other cases equations (7) and (9) must be used.

\section{Fourier's Theorem.}

When the number of observations, $n$, becomes very large, and we write

$$
\theta=2 \pi \frac{r}{n}, \quad d \theta=\frac{2 \pi}{n},
$$

equations (7) may be written

$$
\begin{aligned}
A_{0} & =\frac{d \theta}{2 \pi} \Sigma l, \\
A_{8} & =\frac{d \theta}{\pi} \Sigma(l \cos s \theta), \\
& =\frac{d \theta}{\pi} \Sigma(l \sin s \theta) .
\end{aligned}
$$

In the limiting case, where $n$ is infinite, $d \theta$ becomes an 
infinitesimally small quantity, and the summations may be written as integrals

$$
\left.\begin{array}{l}
A_{0}=\frac{1}{2 \pi} \int_{0}^{2 \pi} l d \theta \\
A_{s}=\frac{1}{\pi} \int_{0}^{2 \pi} l \cos s \theta d \theta \\
B_{s}=\frac{1}{\pi} \int_{0}^{2 \pi} l \sin s \theta d \theta
\end{array}\right\}
$$

If the values of $l$ be given in terms of the time $t$ as argument, and if $t_{0}$ and $t_{0}+T$ be the initial and final times, then the $\theta$ of equations (12) is given by

$$
\theta=2 \pi \frac{t-t_{0}}{T}
$$

Equations (12) correspond to the case where the quantity $l$ is known for an infinite number of values of the argument $\theta$, or $t$; and if $l$ is finite for all values of $t$ between $t_{0}$ and $t_{0}+T$, the integrals of equations (12) will be finite and singlevalued. This conclusion will hold even when $l$, expressed as a function of $t$, has a number of finite discontinuities within the range considered. This is equivalent to a statement of Fourier's Theorem.

Fourier's Theorem states that if $l$ is a function of $\theta$ which is finite and has only a limited number of maxima and minima and of finite discontinuities within the range $0<\theta<2 \pi$, it can be represented by a trigonometric series

$$
A_{0}+\sum_{s=1} A_{s} \cos s \theta+\sum_{s=1} B_{s} \sin s \theta
$$

(where the constants $A_{0}, A_{1}, A_{2}$, etc., are given by equations (12) above) at all points where it is continuous. At points where $l$ is discontinuous the series gives the arithmetic mean of the two values of $l$ at the discontinuity.

This Theorem, so far as it concerns the representation of $l$ by the series at points where $l$ is continuous, follows from equations (12) above. For a full treatment of Fourier's Theorem the reader is referred to any modern textbook of mathematical analysis. 
85. Evaluation of the Coefficients. n a multiple of 4 .

The work of evaluating the coefficients is much simplified when $n$ is a multiple of 4 . Let $n=4 p$, where $p$ is an integer. Then as $r$ takes in succession all values from 0 to $4 p-1, \cos \frac{2 \pi r s}{n}$ and $\sin \frac{2 \pi r s}{n}$ each take the same absolute value four times.

If we write the observations in order, thus,

$$
\begin{array}{llllll}
l_{0} & l_{1} & l_{2} & \ldots & l_{2 p-1} & l_{2 p} \\
& l_{4 p-1} & l_{4 p-2} & \ldots & l_{2 p+1}, &
\end{array}
$$

then the sines which multiply corresponding $l$ 's have opposite signs, while the cosines have the same sign, for all values of $s$. Adding the two rows, we obtain

$$
\begin{array}{lllll}
a_{0} & a_{1} & a_{2} & \ldots & a_{2 p},
\end{array}
$$

and subtracting them, we obtain

$$
\begin{array}{llll}
b_{1} & b_{2} & \ldots & b_{2 p-1}
\end{array}
$$

In dealing with sines, the end terms drop out, since they are multiplied by a factor which becomes zero for all values of $s$.

The normal equations for the coefficients may now be written

$$
\begin{aligned}
& 2 p A_{i}=\Sigma\left(a_{r} \cos r i q\right), \quad \text { where } q=\frac{2 \pi}{n}=\frac{\pi}{2 p}, \\
& 2 p B_{i}=\Sigma\left(b_{r} \sin r i q\right)
\end{aligned}
$$

for all values of $i$ from 0 to $\frac{n}{2}-1$, and

$$
4 p A_{\frac{n}{2}}=\Sigma\left(a_{r} \cos \pi r^{r}\right)=\Sigma(-1)^{r} a_{r}
$$

But

$$
\begin{aligned}
& \cos r i q= \pm \cos (\iota \pi-r i q), \\
& \sin r i q=\mp \sin (\iota \pi-r i q),
\end{aligned}
$$

where the upper or lower sign is taken according as $i$ is even or odd.

We now write the $a$ 's in the form

$$
\begin{array}{llllll}
a_{0} & a_{1} & a_{2} & \ldots & a_{p-1} & a_{p} \\
a_{2 p} & a_{2 p-1} & a_{2 p-2} & \ldots & a_{p+1} . &
\end{array}
$$


Adding the columns, we obtain

and subtracting,

$$
\begin{array}{llllll}
\alpha_{0} & \alpha_{1} & \alpha_{2} & \ldots & \alpha_{p-1} & \alpha_{p},
\end{array}
$$

$$
\alpha_{0}^{\prime} \quad \alpha_{1}^{\prime} \quad \alpha_{2}^{\prime} \quad \ldots \quad \alpha_{p-1}^{\prime} .
$$

Then it follows that for even values of $i$,

$$
2 p A_{i}=\Sigma\left(\alpha_{r} \cos r i q\right), \quad(r=0,1,2, \ldots, p),
$$

and for odd values of $i$,

$$
2 p A_{i}=\Sigma\left(\alpha_{r}^{\prime} \cos r i q\right),
$$

while the first and last coefficients are given by

$$
\begin{aligned}
& 4 p A_{0}=\Sigma\left(\alpha_{r}\right), \\
& 4 p A_{\frac{n}{2}}=\alpha_{0}-\alpha_{1}+\alpha_{2}, \text { etc. }
\end{aligned}
$$

Similarly, writing

$$
\begin{array}{llllll}
b_{1} & b_{2} & b_{3} & \ldots & b_{p-1} & b_{p} \\
b_{2 p-1} & & & & b_{p+1}, &
\end{array}
$$

and adding, we obtain

$$
\beta_{1} \quad \beta_{2} \quad \ldots \beta_{p-1} \beta_{p},
$$

and subtracting, we obtain

$$
\beta_{1}{ }^{\prime} \quad \beta_{2}{ }^{\prime} \quad \ldots \beta_{p-1}^{\prime} .
$$

Then for even values of $i$,

$$
2 p B_{i}=\Sigma\left(\beta_{r}^{\prime} \sin r i q\right),(r=1,2, \ldots, p-1),
$$

and for odd values of $i$,

$$
2 p B_{i}=\Sigma\left(\beta_{r} \sin r i q\right), \quad(r=1,2,3, \ldots, p) .
$$

The equations derived above are collected for convenience of reference.

$$
\begin{aligned}
& 4 p A_{0}=\alpha_{0}+\alpha_{1}+\alpha_{2}+\ldots, \\
& 2 p A_{1}=\alpha_{0}^{\prime}+\alpha_{1}^{\prime} \cos q+\alpha_{2}^{\prime} \cos 2 q+\ldots, \\
& 2 p A_{2}=\alpha_{0}+\alpha_{1} \cos 2 q+\alpha_{2} \cos 4 q+\ldots, \\
& 2 p A_{3}=\alpha_{0}^{\prime}+\alpha_{1}^{\prime} \cos 3 q+\alpha_{2}^{\prime} \cos 6 q+\ldots, \\
& 2 p A_{4}=\alpha_{0}+\alpha_{1} \cos 4 q+\alpha_{2} \cos 8 q+\ldots, \\
& \ldots \ldots \ldots \ldots \ldots \ldots \ldots \ldots \ldots \ldots \ldots \ldots \ldots \ldots \ldots \ldots \ldots \ldots \ldots \ldots \ldots \ldots \ldots \ldots \ldots \ldots \\
& 2 p B_{1}=\beta_{1} \sin q+\beta_{2} \sin 2 q+\ldots, \\
& 2 p B_{2}=\beta_{1}^{\prime} \sin 2 q+\beta_{2}^{\prime} \sin 4 q+\ldots, \\
& 2 p B_{3}=\beta_{1} \sin 3 q+\beta_{2} \sin 6 q+\ldots,
\end{aligned}
$$


These formulae may be used in all cases where $n$ is a multiple of 4. In the special case where $n=12$, or $p=3, q=30^{\circ}$, and the expressions derived above contain at most four terms.

Example 1. Find a trigonometric series which shall represent accurately the following series of determinations of the monthly mean temperature on Ben Nevis during 1902.

$\begin{array}{llll}\text { Jan. } & 23^{\circ} \cdot 8 \mathrm{~F} . & \text { July } & 37^{\circ} \cdot 6 \mathrm{~F} . \\ \text { Feb. } & 22 \cdot 2 & \text { Ang. } & 38 \cdot 0 \\ \text { March } & 25 \cdot 8 & \text { Sept. } & 38 \cdot 4 \\ \text { A pril } & 27 \cdot 1 & \text { Oct. } & 32 \cdot 4 \\ \text { May } & 27 \cdot 6 & \text { Nov. } & 30 \cdot 0 \\ \text { Junc } & 39 \cdot 5 & \text { Dec. } & 25 \cdot 9\end{array}$

\begin{tabular}{|c|c|c|c|c|c|c|c|c|}
\hline$r$ & \multicolumn{2}{|c|}{$l$} & $a$ & $b$ & $\alpha$ & $\alpha^{\prime}$ & $\beta$ & $\beta^{\prime}$ \\
\hline 0 & $23 \cdot 8$ & & $23 \cdot 8$ & & $>61 \cdot 4$ & $-13 \cdot 8$ & $-2 \cdot 2$ & $-5 \cdot 2$ \\
\hline 1 & $22 \cdot 2$ & $25 \cdot 9$ & $48 \cdot 1$ & $-3 \cdot 7$ & $125 \cdot 6$ & $-29 \cdot 4$ & $-15 \cdot 0$ & $6 \cdot 6$ \\
\hline 2 & $25 \cdot 8$ & $30 \cdot 0$ & $55 \cdot 8$ & $-4 \cdot 2$ & $121 \cdot 8$ & $-10 \cdot 2$ & $-5 \cdot 3$ & \\
\hline 3 & $27 \cdot 1$ & $32 \cdot 4$ & 59.5 & -5.3 & $59 \cdot 5$ & & & \\
\hline 4 & $27 \cdot 6$ & $38 \cdot 4$ & $66^{\circ} 0$ & -10.8 & $a_{0}+a_{2}$ & $a_{0}^{\prime}-a_{0}^{\prime}$ & $\beta_{1}-\beta_{2}$ & \\
\hline 5 & $39 \cdot 5$ & $38 \cdot 0$ & $77 \cdot 5$ & 1.5 & ( & & & \\
\hline 6 & $37 \cdot 6$ & & $37 \cdot 6$ & & $=185 \cdot 1$ & & & \\
\hline
\end{tabular}

We have $q=30^{\circ}, \quad \cos 30^{\circ}=866, \quad \cos 60^{\circ}=\cdot 5, \quad \cos 90^{\circ}=0$,

$$
12 A_{0}=183 \cdot 2+185 \cdot 1=368 \cdot 3 \text {. }
$$

$\begin{array}{cccccr}r & \cos \theta & a^{\prime} \cos \theta & r & \cos \theta & a \cos \theta \\ 0 & 1 & -13.8 & 0 & 1 & 61 \cdot 4 \\ 1 & \pm \cdot 866 & \mp 25.46 & 1 & \pm 0.5 & \pm 62.8 \\ 2 & 0.5 & -5.1 & 2 & -0.5 & -60.9 \\ & & & 3 & \mp 1 & \mp 59.5\end{array}$

(Upper sign) $6 A_{1}=-44 \cdot 36 . \quad$ (Upper sign) $6 A_{2}=3.8$.

(Lower sign) $6 A_{5}=6.56 . \quad$ (Lower sign) $6 A_{4}=-2 \cdot 8$.

$6 A_{3}=a_{0}^{\prime}-a_{2}^{\prime}=-3 \cdot 6 . \quad 12 A_{6}=a_{0}-a_{1}+a_{2}-a_{3}=183 \cdot 2-185 \cdot 1=-1 \cdot 9$. 
For the $B$ 's,

$\begin{array}{lcllrl}\gamma & \sin \theta & \beta \sin \theta & \gamma & \sin \theta & \beta^{\prime} \sin \theta \\ 1 & 0.5 & -1.1 & 1 & 0.866 & -4.50 \\ 2 & \pm 0.866 & \mp 12.99 & 2 & \pm 0.866 & \pm 5.71 \\ 3 & 1 & -5.3 & & & \end{array}$

(Upper sign) $6 B_{1}=-19 \cdot 39$. (Upper'sign) $6 B_{2}=1 \cdot 21$.

(Lower sign) $6 B_{5}=\quad 6.59$. (Lower sign) $6 B_{4}=-10.21$.

$$
6 B_{3}=\beta_{1}-\beta_{3}=3 \cdot 1 \text {. }
$$

The required trigonometrical expression is therefore

Temperature $=30^{\circ} \cdot 69-7^{\circ} \cdot 39 \cos \theta+0^{\circ} \cdot 63 \cos 2 \theta-0^{\circ} \cdot 60 \cos 3 \theta$

$-0^{\circ} \cdot 47 \cos 4 \theta+1^{\circ} \cdot 09 \cos 5 \theta-0^{\circ} \cdot 16 \cos 6 \theta$

$-3^{\circ} \cdot 23 \sin \theta+0^{\circ} \cdot 20 \sin 2 \theta+0^{\circ} \cdot 52 \sin 3 \theta-1^{\circ} \cdot 70 \sin 4 \theta$

$+1^{\circ} \cdot 10 \sin 5 \theta$.

A simple check on a portion of the work is obtained by putting $\theta=0$ in this expression. Its value is then

$$
30 \cdot 69-7 \cdot 39+0 \cdot 63-0 \cdot 60-0 \cdot 47+1 \cdot 09-0 \cdot 16=23 \cdot 79 \text {, }
$$

which compares favourably with the measured temperature for January, $23^{\circ} \cdot 8$.

Again, since the formula should give an exact representation of the 12 temperatures, [vv] as derived from equation (11) should be zero.

$$
\begin{aligned}
{[v v]=11734 \cdot 2 } & -12 \times(30 \cdot 69)^{2}-12 \times(0 \cdot 16)^{2} \\
& -6\left\{(7 \cdot 39)^{2}+(0 \cdot 63)^{2}+(0 \cdot 60)^{2}+(0 \cdot 47)^{2}+(1 \cdot 09)^{2}\right. \\
& \left.+(3 \cdot 23)^{2}+(0 \cdot 20)^{2}+(0 \cdot 52)^{2}+(1 \cdot 70)^{2}+(1 \cdot 10)^{2}\right\}=5 \cdot 34 .
\end{aligned}
$$

This is sufficiently near to zero in view of the order of approximation to which the work has been carried out.

Example 2. The relative position of two stars is measured as follows :

Two parallel wires are made to pass each through one star, and the distances $z_{0}, z_{1}, z_{2}, \ldots, z_{11}$ between the two wires are measured when the wires make angles of $0,30^{\circ}, 60^{\circ}, 90^{\circ}, \ldots, 330^{\circ}$ with a fixed axis in their plane. The following 12 measures give the distances $z_{0}, z_{1}$, etc. in terms of one division of the micrometer head; and one division is equal to $0 " 4208$. Find the angular distance of the two stars.

$$
\begin{array}{lll}
z_{0}=50 \cdot 4, & z_{4}=64 \cdot 0, & z_{8}=49 \cdot 6, \\
z_{1}=54 \cdot 3, & z_{5}=61 \cdot 8, & z_{9}=46 \cdot 2, \\
z_{2}=59 \cdot 9, & z_{6}=58 \cdot 9, & z_{10}=46 \cdot 0, \\
z_{3}=62 \cdot 7, & z_{7}=53 \cdot 8, & z_{11}=47 \cdot 2 .
\end{array}
$$

It can easily be shown that $z$ is given by

$$
z_{r}=A_{0}+A_{1} \cos \theta_{r}+B_{1} \sin \theta_{r}, \quad \text { where } \theta_{r}=r .30^{\circ} \text {. }
$$

Then $\rho$, the angular distance between the two stars, is given by

$$
\rho^{2}=A_{1}^{2}+B_{1}^{2}
$$


and we need only evaluate $A_{1}$ and $B_{1}$, following the seheme of the preceding example.

$$
\text { Ans. } \rho=9 \cdot 174=3^{\prime \prime} \cdot 86 \text {. }
$$

Example 3. The following table gives the monthly mean temperatures recorded at Greenwich from 1841 to 1890 . Obtain a complete Fonrier series to represent these values.

$\begin{array}{llll}\text { Jan. } & 38^{\circ} \cdot 5 \mathrm{~F} . & \text { May } 53^{\circ} \cdot 1 \mathrm{~F} . & \text { Sept. } 57^{\circ} \cdot 2 \mathrm{~F} . \\ \text { Feb. } & 39 \cdot 5 & \text { June } 59 \cdot 4 & \text { Oct. } 50 \cdot 0 \\ \text { March } 41 \cdot 7 & \text { July } 62 \cdot 5 & \text { Nor. } 43 \cdot 2 \\ \text { April } 47 \cdot 2 & \text { Aug. } 61 \cdot 6 & \text { Dec. } 39 \cdot 7\end{array}$

\section{Further simplification when $\mathbf{n}$ is a multiple of 8 .}

If $n$ is a multiple of 8 , then $p$ in the equations of page 182 is an even number. It is then found that the work of evaluating the coefficients may be still further shortened, in the case of the even harmonics, while the formulae for the odd harmonics remain unchanged.

Writing the a's in the form

$$
\begin{array}{lllll}
\alpha_{0} & \alpha_{1} & \alpha_{2} & \ldots & \\
\alpha_{p} & \alpha_{p-1} & & \ldots & \alpha_{\frac{p}{2}+1},
\end{array}
$$

and adding

and subtracting

$$
\begin{array}{cccc}
\gamma_{0} & \gamma_{1} & \gamma_{2} & \ldots, \\
\gamma_{0}^{\prime} & \gamma_{2}^{\prime} & \gamma_{2}^{\prime} & \ldots,
\end{array}
$$

we can then write the formulac for the even $A$ 's thus,

$$
\begin{aligned}
& 4 p A_{0}=\gamma_{0}+\gamma_{1}+\gamma_{2}+\ldots, \\
& 2 p A_{2}=\gamma_{0}^{\prime}+\gamma_{1}^{\prime} \cos 2 q+\gamma_{2}^{\prime} \cos 4 q+\ldots, \\
& 2 p A_{4}=\gamma_{0}+\gamma_{1} \cos 4 q+\gamma_{2} \cos 8 q+\ldots, \\
& 2 p A_{6}=\gamma_{0}^{\prime}+\gamma_{1}^{\prime} \cos 6 q+\gamma_{2}^{\prime} \cos 12 q+\ldots .
\end{aligned}
$$

Again, writing the $\beta$ 's in the form

$$
\begin{array}{lllll}
\beta_{1}^{\prime} & \beta_{2}^{\prime} & \ldots & \beta_{\frac{p}{2}-1} & \beta_{p} \\
\beta_{p-1}^{\prime} & \beta_{p-2}^{\prime} & \ldots & \beta_{\frac{p}{2}+1},
\end{array}
$$

and adding

and subtracting

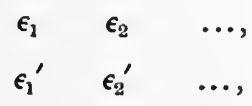


we can then write the formulae for the even $B$ 's thus,

$$
\begin{aligned}
& 2 p B_{2}=\epsilon_{1} \sin 2 q+\epsilon_{2} \sin 4 q+\ldots, \\
& 2 p B_{4}=\epsilon_{1}{ }^{\prime} \sin 4 q+\epsilon_{2}^{\prime} \sin 8 q+\ldots, \\
& 2 p B_{6}=\epsilon_{1} \sin 6 q+\epsilon_{2} \sin 12 q+\ldots
\end{aligned}
$$

\section{Practical method of investigating periodicities.}

In practice, we should not endeavour to find the amplitude of a periodic term from observations extending over one period only. Thus a periodic term of one year would only be investigated by means of observations extending over a large number of years. The work is then arranged as foliows.

Let $u_{0}, u_{1}, u_{2}$, etc. be a series of observations taken at equal intervals of time $\alpha$; and suppose it is required to investigate a possible period $p \alpha$. The $u$ 's are written in rows of $p$ each, as follows:

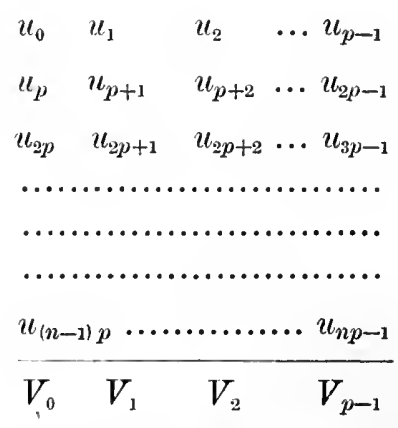

We shall suppose that there are sufficient observations, to form $n$ rows of $p$ each. The columns are added, yielding the sums $V_{0}, V_{1}, \ldots, V_{p-1}$. The sums $V_{0}, V_{1}$, etc. are then analysed harmonically by the methods outlined above, yielding the expression

$$
V=A_{0}+A_{1} \cos \theta+B_{1} \sin \theta,
$$

where $V$ takes the value $V_{r}$, when $\theta=\frac{2 \pi r}{p}$,

$$
A_{1}=\frac{2}{p} \sum_{r=0}^{p-1} V_{r} \cos \frac{2 \pi r}{p}=\frac{2}{p} \sum_{r=0}^{p n-1} u_{r} \cos \frac{2 \pi r}{p},
$$




$$
\begin{aligned}
& B_{1}=\frac{2}{p} \sum_{r=0}^{p-1} V_{r} \sin \frac{2 \pi r}{p}=\frac{2}{p} \sum_{r=0}^{m-1} u_{r} \cos \frac{2 \pi r}{p}, \\
& A_{0}=\frac{1}{p} \sum_{r=0}^{p-1} V_{r} .
\end{aligned}
$$

When it is only required to investigate a simple periodicity of period $p$ intervals, it is not necessary to carry the Fourier expansion beyond the terms in $\cos \theta$ and $\sin \theta$.

The amplitude in the $V$ 's of the period $p a$ is

$$
\left(A_{1}^{2}+B_{1}^{2}\right)^{\frac{1}{2}} \text {. }
$$

The amplitude of the period $p a$ in the original observations $u_{0}, u_{1}$, etc. will be

$$
\frac{1}{n}\left(A_{1}^{2}+B_{1}^{2}\right)^{\frac{1}{2}}
$$

For if

$$
t=a_{0}+a_{1} \cos \theta+b_{1} \sin \theta+\ldots,
$$

where $t$ takes the value $u_{r}$ for $\theta=\frac{2 \pi r}{p}$, then each of the periodic terms is repeated exactly down the columns of the table of $u$ 's. The amplitude of the variation in the $V$ 's is thus $n$ times the amplitude of the same period in the u's. Thus we may write

$$
\left(a_{1}^{2}+b_{1}^{2}\right)^{\frac{1}{2}}=\frac{1}{n}\left(A_{1}^{2}+B_{1}^{2}\right)^{\frac{1}{2}} .
$$

The right-hand side of this equation gives the required amplitude.

The method has the advantage of destroying to a certain extent accidental errors in individual $u$ 's. Periods of $p$ intervals or of any exact sub-multiple of this period will be intensified $n$-fold in the sequence $V_{0}, V_{1}, V_{2}$, etc.; while other periodicities will tend to be destroyed by the process of addition. For the latter periodicities will occur with different phases in the horizontal rows of the table of $u$ 's, and the terms so produced will tend to annul one another on addition of the columns, provided $n$ is large, and the periods concerned be not too nearly equal to the subperiods of $p$ intervals. 
The figures given in Ex. 3, page 185, afford an example of this method. The temperature of $38^{\circ} \cdot 5$ given for January is the mean of the January temperature for 50 years. This corresponds to the nethod shown in the table on page $186^{\circ}$, except that the $V$ 's are all divided by $n$, the result being treated as the mean annual variation. In practice it will sometimes be found convenient to analyse the quantities $V_{0}, V_{1}$, etc. as they stand, while in other cases it will be found preferable to divide the $V$ 's by $n$, treating the result as the mean variation of period $p \alpha$.

Further, it should be noted that if the same quantity be added to, or subtracted from, all the terms which we desire to analyse harmonically, only the constant $A_{0}$ is affected, the periodic terms being unaffected. It is sometimes useful to remember this property, as the numbers with which we have to deal can often be reduced in magnitude by subtracting the same amount from all the terms. Thus in Ex. 3, p. 185, we should obtain the same periodic terms if we subtracted $38^{\circ} .5$ from all the terms before proceeding further.

Tables for facilitating the evaluation of $\Sigma V_{r} \cos r \theta, \Sigma V_{r} \sin r \theta$ have been drawn up by H. H. Turner, and published under the title Tables for Facilitating the Use of Harmonic Analysis (Oxford Univ. Press).

A large number of instruments and graphical methods have been devised for performing the evaluation of the coefficients of the terms of the Fourier series for any given set of observations. For a description of these instruments and methods, as well as of other instruments, tables, etc., the reader is referred to the Handbook of the Napier Tercentenary Celebration of 1914, published by Messrs G. Bell and Sons, under the title of Modern Instruments of Calculation. This work is a mine of information concerning calculating instruments of all kinds.

Example 1. Find the first four harmonics in the Fourier series for the following 24 nurnbers:

$\begin{array}{rrrrrrrrrrrr}97 & 94 & 100 & 110 & 103 & 101 & 85 & 101 & 115 & 91 & 106 & 106 \\ 102 & 113 & 102 & 105 & 99 & 112 & 81 & 100 & 106 & 94 & 83 & 94\end{array}$

In order to decrease the numbers with which we have to deal, it is advisable to subtract 80 from the numbers given. This only affects the constant term, and can be allowed for later.

The numbers are written in the order suggested in $\$ \S 85,86$. 


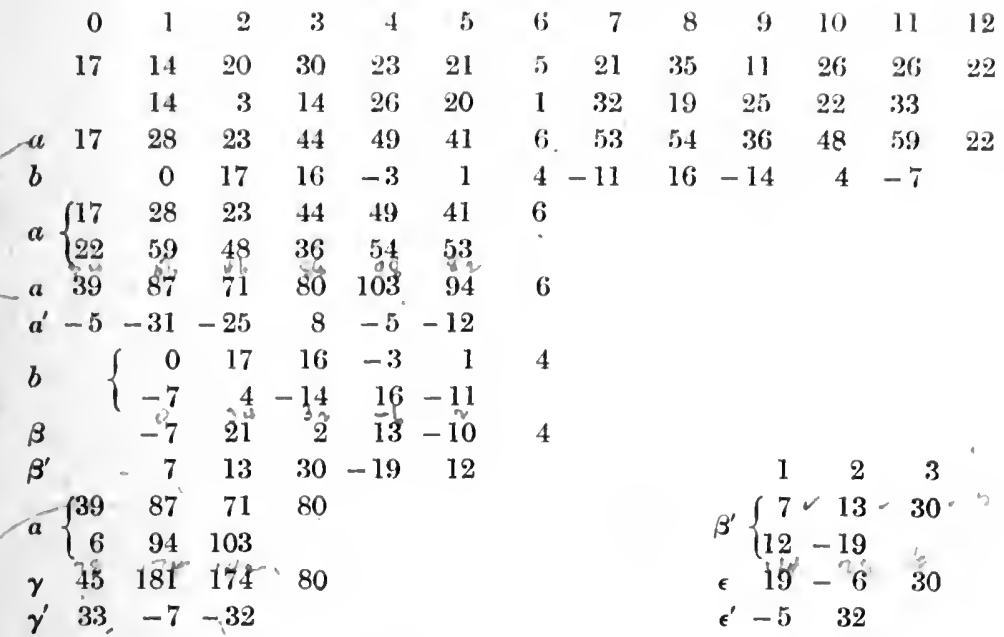

$24 A_{0}=45+181+174+80=480$,

$12 A_{1}=-5-31 \cos 15^{\circ}-25 \cos 30^{\circ}+8 \cos 45^{\circ}$

$-5 \cos 60^{\circ}-12 \cos 75^{\circ}=-56 \cdot 6$,

$12 A_{2}=33-7 \cos 30^{\circ}-32 \cos 60^{\circ}=11$,

$12 A_{3}=-5-31 \cos 45^{\circ}-25 \cos 90^{\circ}+8 \cos 135^{\circ}$

$-5 \cos 180^{\circ}-12 \cos 225^{\circ}=-19 \cdot 1$,

$12 A_{4}=45+181 \cos 60^{\circ}+174 \cos 120^{\circ}+80 \cos 180^{\circ}=-31.5$.

$12 B_{1}=-7 \sin 15^{\circ}+21 \sin 30^{\circ}+2 \sin 45^{\circ}+13 \sin 60^{\circ}$

$-10 \sin 75^{\circ}+4 \sin 90^{\circ}=15 \cdot 7$,

$12 B_{2}=19 \sin 30^{\circ}-6 \sin 60^{\circ}+30 \sin 90^{\circ}=34 \cdot 3$,

$12 B_{3}=-7 \sin 45^{\circ}+21 \sin 90^{\circ}+2 \sin 135^{\circ}+13 \sin 180^{\circ}$

$-10 \sin 225^{\circ}+4 \sin 270^{\circ}=10 \cdot 5$,

$12 B_{4}=-5 \sin 60^{\circ}+32 \sin 120^{\circ}=23 \cdot 4$.

The above figures yield $A_{0}=20$. In order to correct for the original subtraction of 80 from all the figures analysed, we now add 80 to this value of $A_{0}$. The Fourier series then reads

$$
\begin{array}{r}
100-4 \cdot 7 \cos \theta+0.9 \cos 2 \theta-1 \cdot 6 \cos 3 \theta-2 \cdot 6 \cos 4 \theta \\
+1.3 \sin \theta+2.8 \sin 2 \theta-0.9 \sin 3 \theta+2 \cdot 0 \sin 4 \theta,
\end{array}
$$

where $\theta=0,15^{\circ}, 30^{\circ}$, etc. for the first, second, and third figures respectively.

Example 2. Find the first harmonic term in the Fourier series representing the following 20 quantities:

$\begin{array}{rrrrrrrrrr}88 & 124 & 103 & 90 & 49 & 168 & 99 & 62 & 104 & 97 \\ 51 & -28 & -1 & 42 & 55 & 59 & 99 & 38 & 122 & 37\end{array}$


Since only the coefficients $A_{1}$ and $B_{1}$ are required, the work may be considerably shortened.

\begin{tabular}{|c|c|c|c|c|c|c|c|c|c|c|c|}
\hline & 0 & 1 & 2 & 3 & 4 & 5 & 6 & 7 & 8 & 9 & 10 \\
\hline & 88 & 124 & 103 & 90 & 49 & 168 & 99 & 62 & 104 & 97 & 51 \\
\hline & & 37 & 122 & 38 & 99 & 59 & 55 & 42 & -1 & -28 & \\
\hline & $(88$ & 161 & 225 & 128 & 148 & 227 & 154 & 104 & 103 & 69 & 1 \\
\hline\{ & $\{51$ & 69 & 103 & 104 & 154 & & & & & & \\
\hline$a^{\prime}$ (Subtract) & 37 & 92 & 122 & 24 & -6 & & & & & & \\
\hline 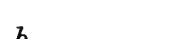 & & $\int 87$ & -19 & 52 & -50 & 109 & {$[44$} & 20 & 105 & 125] & \\
\hline b & & $\{125$ & 105 & 20 & 44 & & & & & & \\
\hline$\beta(\mathrm{Add})$ & & 212 & 86 & 72 & -6 & 109 & & & & & \\
\hline
\end{tabular}

$10 A_{1}=37+92 \cos 18^{\circ}+122 \cos 36^{\circ}+24 \cos 54^{\circ}-6 \cos 72^{\circ}=235 \cdot 3$,

$10 B_{1}=212 \sin 18^{\circ}+86 \sin 36^{\circ}+72 \sin 54^{\circ}-6 \sin 72^{\circ}+109 \sin 90^{\circ}=277 \cdot 5$.

Hence the required terms are

$23 \cdot 5 \cos \theta+27 \cdot 7 \sin \theta$. 


\section{CHAP'TER XII}

\section{THE PERIODOGRAM}

\section{Hidden Periodicities.}

In certain classes of observations, the length of the main period becomes obvious as soon as the observations are examined; e.g. the period of the semi-diurnal tide can be deduced from a comparatively small number of observations. Once the length of the period is known, the methods of the preceding chapter can be immediately applied to deduce the amplitude and phase. But when the length of the period is unknown, and cannot be deduced in a simple manner, the difficulties of the investigation are enormously increased. Thus, if we consider records of temperature extending over a large number of years, we shall find that, once the effect of the annual period and its harmonics has been removed, the resulting records show no obvious periodicity, though they are probably due to a number of superposed periodicities, with certain accidental variations added on. In meteorological phenomena generally, the changes from day to day appear to be so arbitrary, that one is forced to the conclusion that, whatever periodicities may underlie the phenomena, they will be very largely masked by apparently accidental variations. The methods considered in the present chapter aim at unmasking such underlying periodicities, and determining their amplitudes and phases.

It has already been shown in Chapter XI that it is always possible to find a Fourier series which shall represent with any required degree of accuracy any given set of numbers; but it must not be assumed that the Fourier series is always an accurate representation of the physical laws underlying the phenomena. For it is obvious that even a number of quantities distributed at random can be represented by a Fourier series; though in such a 
case the use of the series might lead to the false impression that the phenomena under consideration were due to the combined action of a number of purely periodic physical causes. The real difficulty lies, not so much in finding a Fourier series to fit the observations, as in determining which of a large number of possible periods have sufficiently great amplitudes to be regarded as having real physical significance.

\section{The Periodogram.}

The Periodogram method of searching for periodicities consists essentially in finding (by the method of $\S 87$, or otherwise) the amplitudes of a large number of trial periods. The trial periods which yield the greatest amplitudes will yield approximations to the most probable periodicities. It will be proved later that if a trial period $T$ should fall near one of the actual periods of the observations, the resulting amplitude $R$ yielded by the method of $\S 87$ will be considerably greater than if the trial period $T$ were considerably removed from the actual periods. Thus if $R^{2}$ be plotted for different values of $T$, the points of maxima of the curve will yield the most likely periods. If $N$ observations be used in deriving $R$ for the period $T$, it will be found in some ways preferable to plot $R^{2} N$ for different values of $T$; but in practice if we had say 600 readings to hand for a periodogram investigation we should use all or practically all of the readings for investigating all trial periods. Thus $N$ will never in practice vary considerably, and it is sufficient to plot $R^{2}$ for different values of $T$. The rather ill-defined maxima of this curve will yield approximations to the most likely periods. More accurate values of these periods will afterwards be derived by what is called the Secondary Analysis $(§ 91)$. Finally it is necessary to consider how great the amplitude of any of these periods must be, in order that we may be certain that it. did not arise from a purely chance distribution of the quantities considered.

It is of course impracticable to find $R^{2}$ for all possible values of $T$, and Schuster has shown that, if a period $T$ has been investigated, the nearest period to this which need be investigated is $T^{\prime}$, given by the equation

$$
n\left(T-T^{\prime}\right)= \pm \frac{1}{4} T .
$$


This limit is set by the fact that any two periods which may be investigated should be independent, and two near periods will begin to be independent when there is a final difference of phase of a quarter-period. This condition leads to the above equation, where $n$ is the number of periods ' $T$ ' used in the investigation.

\section{Form of the Periodogram for one simple period.}

Suppose the observations to be analysed could be represented by a single periodic function $R^{\prime} \cos (\kappa t-\epsilon)$, where $\kappa=\frac{2 \pi}{T^{\prime}}, T^{\prime}$ being the true period of the series. Let ' $T$ ' be one of the primary periods for which the observations have been analysed, and let $T^{\prime}=\frac{2 \pi}{g}$. Then if the number of observations be large, the summations used in the usual harmonic analysis can be replaced by integrations. The coefficients of the first two terms in the Fourier sequence then become*

$$
\begin{aligned}
& \frac{1}{2} n T^{\prime} A_{1}=R^{\prime} \int_{0}^{n T} \cos (\kappa t-\epsilon) \cos g t d t, \\
& \frac{1}{2} n T^{\prime} B_{1}=R^{\prime} \int_{0}^{n T} \cos (\kappa t-\epsilon) \sin g t d t,
\end{aligned}
$$

where $n$ is the total number of complete periods $T$ used in the analysis. Integrating these expressions, and remembering that

we obtain

$$
g n ' T=2 \pi n,
$$

$$
\begin{aligned}
\frac{1}{2} n T A_{1} & =\frac{1}{2} R^{\prime} \int_{0}^{n T}\{\cos (\overline{\kappa+g} t-\epsilon)+\cos (\overline{\kappa-g} t-\epsilon)\} d t \\
& =R^{\prime} \frac{2 \kappa}{\kappa^{2}-g^{2}} \sin \frac{1}{2} \kappa n T^{\prime} \cos (\kappa n T-\epsilon), \\
\frac{1}{2} n T B_{1} & =-R^{\prime} \frac{2 q}{\kappa^{2}-g^{2}} \sin \frac{1}{2} \kappa n T \sin (\kappa n T-\epsilon) .
\end{aligned}
$$

Or, if $R$ be the amplitude of the period $T$ yielded by the primary analysis,

$$
\begin{aligned}
& \frac{R}{R^{\prime}}=\frac{4}{\left(\kappa^{2}-g^{2}\right) n T}, \sin \frac{1}{2} \kappa n T \cdot\left\{\kappa^{2} \cos ^{2}(\kappa n T-\epsilon)+g^{2} \sin ^{2}(\kappa n T-\epsilon)\right\}^{\frac{1}{2}} \\
& \text { Putting } \quad \gamma=\frac{1}{2}(\kappa-g) n T=\pi n\left(\frac{g}{\kappa}-1\right), \\
& \text { * Cf. equations (12), page } 180 .
\end{aligned}
$$

B. 0 . 
and

$$
\sin \frac{1}{2} \kappa n T=\sin \left\{\frac{1}{2}(\kappa-g) n T+\frac{1}{2} g n T\right\}=\sin (\gamma+n \pi)=(-1)^{n} \sin \gamma,
$$

we obtain the relation

$$
\frac{R}{R^{\prime}}=\frac{\sin \gamma}{\gamma} \times \frac{2\left\{\kappa^{2} \cos ^{2}(2 \gamma-\epsilon)+g^{2} \sin ^{2}(2 \gamma-\epsilon)\right\}^{\frac{1}{2}}}{\kappa+g} .
$$

When $\kappa$ and $g$ are equal or very nearly equal, this reduces to

Since

$$
\frac{R}{R^{\prime}}=\frac{\sin \gamma}{\gamma}
$$

$$
\gamma=\frac{1}{2}(\kappa-g) n T=\pi n \frac{q-\kappa}{\kappa}=\frac{1}{2} \kappa n T-\pi n=\pi n\left(\frac{T}{T^{\prime}}-1\right),
$$

it follows that $\frac{\sin \gamma}{\gamma}$ only has appreciable values when $g-\kappa$ is small. For the function $\frac{\sin \gamma}{\gamma}$ has its maximum at $\gamma=0$, and decreases to zero at $\gamma=\pi$, after which it has a succession of maxima and minima whose amplitudes are small.

Considering the equation

$$
\frac{R^{2}}{R^{\prime 2}}=\frac{\sin ^{2} \gamma}{\gamma^{2}} \times \frac{4\left\{\kappa^{2} \cos ^{2}(2 \gamma-\epsilon)+g^{2} \sin ^{2}(2 \gamma-\epsilon)\right\}}{(\kappa+g)^{2}},
$$

we see that the second factor only varies slowly, so that the curve representing the values of $\frac{R^{2}}{R^{\prime 2}}$ for different values of $\gamma$ is not appreciably altered, so far as its general form is concerned, when this factor is neglected. The general distribution of $\frac{R^{2}}{R^{\prime 2}}$ is approximately of the form of $\frac{R^{2}}{R^{\prime 2}}=\frac{\sin ^{2} \gamma}{\gamma^{2}}$ at points near $\gamma=0$. The curve will show a broad band at $\gamma=0$, and on each side of this band a succession of other bands of rapidly decreasing intensity. The result is analogous with the formation of diffraction bands (fig. 12). The greater the total number $n$ of periods used, the nearer will the diffraction bands crowd together on each side of the principal band, and the narrower will the principal band be.

It is thus seen that a curve showing $R^{2}$ for different values 
of $\gamma$, which is of precisely the same form as the curve representing $R^{2}$ for different values of $T\left[\right.$ since $\left.\gamma=\pi n\left(\frac{T}{T^{\prime}}-1\right)\right]$, shows a broad band at $\gamma=0$ or $T=T^{\prime}$, and a succession of subsidiary bands of small maximum ordinate on each side of this band. Since it was assumed in the first place that the period $T^{\prime}$ was the only true period, the curve represents the effect of the true period ' $T$ ' upon

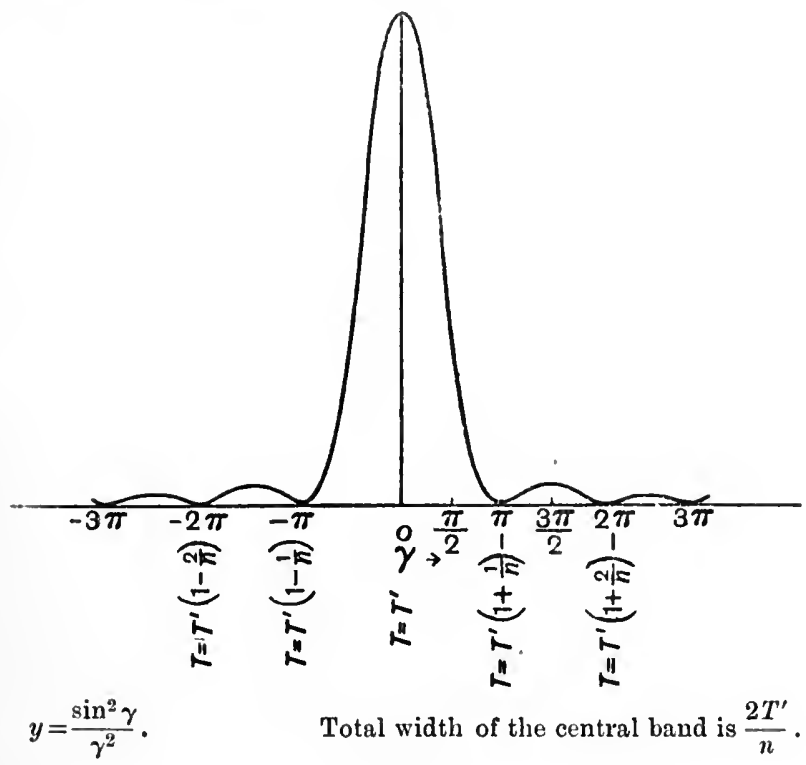

Fig. 12.

the square of the amplitude of other trial periods analysed by the periodogram; and it has been shown that this effect is negligible except for such trial periods $T$ as will yield small values of $\gamma$. The central band does not spread beyond

$$
\gamma= \pm \pi, \text { or } n\left(\frac{T}{T^{\prime}}-1\right)= \pm 1
$$

The limits of the central band are therefore

$$
T=T^{\prime}\left(1 \pm \frac{1}{n}\right)
$$

where $n$ is the total number of complete periods considered in the investigation. 
Thus we were justified in the assumption made in $\S 89$ that the periodogram spreads a periodicity into a band. It has been shown also that the width of the band can be decreased by increasing the number of complete periods considered in the analysis. But since $\frac{\sin ^{2} \gamma}{\gamma^{2}}$ only changes slowly in the neighbourhood of $\gamma=0$, the maximum shown in the periodogram will be flat, and the actual point of maximum will therefore be ill-defined. It must therefore not be expected that the primary analysis of the periodogram should yield the exact value of the period.

\section{Secondary Analysis.}

When the periodogram has shown the presence of a true periodicity in the neighbourhood of a primary period $T$, the true period $T^{\prime \prime}$ can be obtained by analysing harmonically each period $T$, or the sums of groups of $m$ periods. It will be supposed that the number of observations is sufficiently large to permit of our replacing summations by integrals.

In the first place suppose the observations $u_{0}, u_{1}$, etc. can be accurately represented by a simple period

$$
u=C+R^{\prime} \cos (\kappa t-\epsilon) .
$$

Let the $n$ complete periods be divided into groups of $m$ periods, and let the first two terms in the harmonic analysis of the sth group be

Then

$$
A_{s} \cos g t+B_{8} \sin g t, \text { where } g=\frac{2 \pi}{T} .
$$

$$
\begin{aligned}
\frac{1}{2} m T A_{s} & =\int_{(s-1) m T}^{s m T} u \cos g t d t=R^{\prime} \int_{(s-1) m T}^{s m T} \cos (\kappa t-\epsilon) \cos g t d t \\
& =\frac{1}{2} R^{\prime} \int_{(s-1) m T}^{s m T}-\{\cos (\overline{\kappa+g} t-\epsilon)+\cos (\overline{\kappa-g} t-\epsilon)\} d t \\
\frac{m T A_{s}}{R^{\prime}} & =\left(\frac{1}{\kappa+g}+\frac{1}{\kappa-g}\right) \sin (s \kappa m T-\epsilon) \\
& \quad-\left(\frac{1}{\kappa+g}+\frac{1}{\kappa-g}\right) \sin (\overline{s-1} \kappa m T-\epsilon) \\
& =\frac{2 \kappa}{\kappa^{2}-g^{2}} \sin \frac{1}{2} \kappa m T \cos \left\{\frac{1}{2}(2 s-1) \kappa m T-\epsilon\right\} .
\end{aligned}
$$


Similarly

$$
\frac{m T B_{s}}{R^{\prime}}=\frac{-2 g}{\kappa^{2}-g^{2}} \sin \frac{1}{2} \kappa m T \sin \left\{\frac{1}{2}(2 s-1) \kappa m T-\epsilon\right\} .
$$

If $\phi_{s}$ be the phase of maximum in this group

$$
\tan \phi_{s}=-\frac{g}{\kappa} \tan \left\{\frac{1}{2}(2 s-1) \kappa m T-\epsilon\right\} .
$$

It has already been shown that if $T^{\prime \prime}$ be the point of maximum of the band in the periodogram, $T$ for different points on the band will lie between $T^{\prime \prime}\left(1 \pm \frac{1}{n}\right)$. It has been supposed that the periodogram has shown that $T^{\prime \prime}$ lies near $T$, or that $T$ is well within the limits $T^{\prime}\left(1 \pm \frac{1}{n}\right)$. Hence it follows that since $\frac{g}{\kappa}=\frac{T^{\prime}}{T^{\prime}}$, this fraction will differ from unity by less than $\frac{1}{n}$. Since $n$ will be fairly large, we may write unity instead of $\frac{g}{\kappa}$ in the above equation. Then

$$
\text { or } \quad \begin{aligned}
\tan \phi_{8} & =-\tan \left\{\frac{1}{2}(2 s-1) \kappa m T-\epsilon\right\}, \\
\phi_{8} & =2 \pi m s-\frac{1}{2}(2 s-1) \kappa m T+\epsilon .
\end{aligned}
$$

This is a linear function of $s$, and therefore $\phi_{s}$ should increase uniformly from group to group. If $\beta$ be the increase of phase per group, then

or

$$
\begin{aligned}
\beta & =2 \pi m-\kappa m T \\
& =2 \pi m\left(1-\frac{T}{T^{\prime \prime}}\right), \\
T^{\prime} & =\frac{T}{1-\frac{\beta}{2 \pi m}} .
\end{aligned}
$$

Hence if the progression of phase per group can be determined when the observations are grouped $m$ periods of $T$ together, and the sum of the groups analysed, the true period can be deduced accurately.

In practice the work is conducted as follows. It is supposed that the primary analysis has shown the existence of a true periodicity near the trial period $T$, where $T$ is equal to $p$ of the intervals between successive observations. The available observations are arranged in a table containing $n$ rows and 
$p$ columns, as in Chap. XI, $\S 87$. The $n$ rows are divided up into groups of $m$ each, and the sums of the columns in each group written down. These sums are then analysed harmonically, either by the methods of $\S 87$ or by the use of a harmonic analyser. Let the first two terms in the Fourier sequence for the sth row be

$$
A_{s} \cos \theta+B_{s} \sin \theta \text {. }
$$

Then the phase $\phi_{s}$ of this row is evaluated by means of the formula

$$
\tan \phi_{8}=\frac{B_{8}}{A_{s}} .
$$

The phase $\phi_{s}$ is evaluated for all the groups. The progression from group to group, $\beta$, can then be immediately evaluated. The method of procedure may perhaps be seen most easily by the consideration of a simple example.

The primary analysis having shown the existence of a period of 19 to 20 months in eertain temperature records, it was required to find a more aceurate estimate of the length of the period. As the records conveniently available extended over about 35 years, or 420 months, the temperatures were written down in periods of 20 months, forming a table of 21 rows. The rows were then arranged in groups of three periods and added, yielding seven groups of three periods each. The sums for each group were then analysed as far as the first harmonic term only. The results are given in the following table:

\begin{tabular}{|c|r|r|r|r|}
\hline Group & \multicolumn{1}{|c|}{$A_{s}$} & \multicolumn{1}{c|}{$B_{s}$} & $\frac{B_{s}}{A_{s}}$ & \multicolumn{1}{c|}{$\phi_{s}$} \\
\hline 1 & $235 \cdot 3$ & $277 \cdot 5$ & $1 \cdot 18$ & $50^{\circ}$ \\
2 & $367 \cdot 8$ & $-10 \cdot 0$ & -.027 & $-1 \cdot 5^{\circ}$ \\
3 & $371 \cdot 4$ & $53 \cdot 4$ & $\cdot 144$ & $8^{\circ}$ \\
4 & -44.6 & $-124 \cdot 8$ & $2 \cdot 79$ & $250^{\circ}$ \\
5 & $-206 \cdot 1$ & -116.7 & $\cdot 57$ & $209^{\circ}$ \\
6 & 140.8 & $-177 \cdot 8$ & $-1 \cdot 27$ & $-52^{\circ}$ \\
7 & $-327 \cdot 3$ & $87 \cdot 1$ & $-\cdot 27$ & $165^{\circ}$ \\
\hline
\end{tabular}


The progression of phase fiom group to group is not obvious from this table, but may be evaluated by the following graphical method (see fig. 13). For each group plot the phase of maximun,

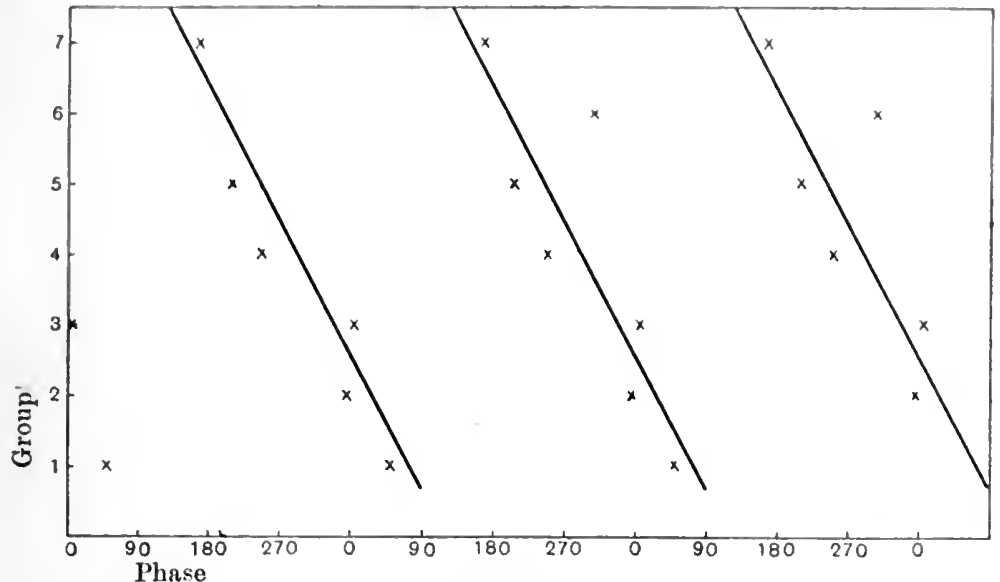

Fig. 13.

remembering that the addition of any multiple of $\pm 360^{\circ}$ to the phase makes no difference to the trigonometrical expression. The points in the diagram group themselves about a number of parallel straight lines, whose slope corresponds to a decrease of phase of $36^{\circ}$ from one group to the next. The corrected period is therefore

$$
\frac{20}{1+\frac{36}{3 \times 360}} \text { months }=\frac{600}{31} \text { months }=19: 35 \text { months. }
$$

\section{Fourier Series for Random Distribution of Obser-} vations.

If the observed values be distributed at random in accordance with the normal law, the number of observations which will differ from the mean by an amount which is between $\beta$ and $\beta+d \beta$ will be $\frac{2 h N}{\sqrt{\pi}} e^{-h^{2} \beta^{2}} d \beta$, where $\dot{h}$ is a constant and $N$ is the total number of observations.

Schuster * has shown that if the observations be represented by a Fourier series of the form

$$
u=a_{0}\left\{1+\rho_{1} \cos \left(\theta-\phi_{1}\right)+\rho_{2} \cos 2\left(\theta-\phi_{2}\right)+\ldots\right\},
$$

* Terrestrial Magnetism, Vol. mr, p. 22. 
then the probability that any coefficient $\rho$ shall be between $\rho$ and $\rho+d \rho$ is

$$
N h^{2} e^{-\frac{1}{2} N h^{2} \rho^{2}} \rho d \rho .
$$

Hence the probability that $\rho$ exceeds $z$ is

$$
N h^{2} \int_{z}^{\infty} e^{-\frac{1}{2} N h^{2} \rho^{2}} \rho d \rho=e^{-\frac{1}{2} N h^{2} z^{2}}
$$

The mean value of $\rho^{2}$ is

$$
N h^{2} \int_{0}^{\infty} e^{-\frac{1}{2} N h^{2} \rho^{2}} \rho^{3} d \rho, \text { or } \frac{2}{N h^{2}} .
$$

The probability that $\rho^{2}$ shall exceed $\kappa$ times its mean value, or $\frac{2 \kappa}{N h^{2}}$, is the same as the probability that $\rho$ shall exceed $\sqrt{\frac{2 \kappa}{N h^{2}}}$, and is therefore $e^{-\kappa}$. As this result will be found to be of considerable importance, a table of values of $e^{-\kappa}$ is here appended.

\begin{tabular}{|c|c|c|c|}
\hline$\kappa$ & $e^{-\kappa}$ & $\kappa$ & $e^{-\kappa}$ \\
\hline 1 & $\cdot 3679$ & 6 & $2 \cdot 4 \times 10^{-3}$ \\
2 & $\cdot 1353$ & 8 & $3.35 \times 10^{-4}$ \\
3 & $\cdot 0498$ & $4.54 \times 10^{-5}$ \\
10 & $6 \cdot 14 \times 10^{-6}$ \\
4 & $\cdot 0183$ & 12 & $1 \cdot 13 \times 10^{-7}$ \\
\hline 5 & .00674 & 16 & \\
\hline
\end{tabular}

This table may be interpreted thus:-The chance of obtaining for the square of a Fourier coefficient a value greater than three times its expectancy or mean value is 0498 , or about 1 in 20 . So that, if on analysing a series of observations we obtain a coefficient whose square is more than three times the expectancy, we can state that the probability that it is produced by a chance distribution of the quantities analysed is $\frac{1}{20}$. If the square of the Fourier coefficient be 12 times its expectancy, the probability that it is produced by a chance distribution is 1 in 160,000 . 


\section{Application to the Periodogram.}

If the amplitude of a period of $p$ intervals (i.e. of period $T^{\prime}=p x$ ) be evaluater as in $\$ \$ 7$, by writing the observations in 1 rows of $p$ each, the number of observations used is $p n$. The nean value of $R^{2}$, or its expectancy if the observations are distributed at random, has been shown above to be $\frac{2}{n p h^{2}}$, where $h$ is an unknown constant. The ratio of $R^{2}$ to its expectancy is $\frac{R^{2} n p l l^{2}}{2}$. If we let

$$
S=R^{2} p m,
$$

and draw a curve to represent the value of $S$ for different values of the period $T$, where $T^{\prime}=p a$, then the ordinate $S$ will always be proportional to the ratio of $R^{2}$ to its expectancy. The area between the curve and the axis of ' $T$ ' is called the periodogram.

If the total number of observations used in investigating all the periods (i.e. $p n$ ) should be sensibly the same, then we may define the ordinate $S$ by the equation

$$
S=R^{2}
$$

In practice $p n$ will not vary within wide limits, and whichever definition of $S$ we take, we may say that the points of maxima of $S$ yicld the most likely periods.

If the period of $p$ intervals investigated above were not real, there would be no simple phase-relation between the terms in the same column of the table of $u$ 's in $\$ 87$, and the sums of the columns, $V_{0}, V_{1}, V_{2}$, etc., might be regarded as being independent of each other, or, in short, might be regarded as a random distribution. So that if $R^{2}$ be evaluated for a range of values of $T$ for which no real period exists, its value should on the whole be equal to its expectancy on the hypothesis of a random distribution of the quantities analysed. For such a range of values of $T$, the ordinate $S$ of the periodogram should show a random distribution about a mean value $\bar{S}$, independent of the value of $T$. It is generally found in practice that the periodogram shows regions where $S$ is relatively small, and the curve has no clearly-defined maxima; and this may be assumed to show the absence of any periodicities within this range. The mean value of $S$ over such a region will yield the value of $\bar{S}$, corresponding to the fact that on the whole $R^{2}$ is equal to its expectancy. 
If $S$ be a maximum ordinate of the periodogram, corresponding to the period $T$, it follows that the ratio of $R^{2}$ to its expectancy is $\frac{S}{\bar{S}}$. Hence the probability that $R^{2}$ is produced by a random distribution is $e^{-\frac{S}{S}}$. If we are satisfied with odds of 1000 to 1 in favour of the period $T$ being real, reference to the table of values of $e^{-\kappa}$ given above shows that $\frac{S}{\bar{S}}$ must be at least equal to 7 . If we are only satisfied with odds of 160,000 to $1, \frac{S}{\bar{S}}$ must be at least equal to 12 .

Thus the periodogram itself will yield a test of the reality of the periods corresponding to the maxima of the curve. When the number $p n$ of observations used in investigating periods of different length is not subject to any considerable variations, the ordinate of the periodogram may be taken equal to $R^{2}$. It is only when $p n$ varies considerably for different periods that it is necessary to include $p n$ as a factor, and define the ordinate of the periodogram as

$$
S=R^{2} p n
$$

\section{Schuster's Investigations.}

The periodogram in its original form was developed by Schuster in a paper entitled* "The Investigation of Hidden Periodicities." In this paper the ordinate of the periodogram corresponding to a period $T$ was defined as the amplitude derived for that period. With our previous notation, $S=R$.

In a later paper $\dagger$, Schuster gave a further analytical development of the periodogram, taking the ordinate to be the square of the amplitude, or $S=R^{2}$.

In a third paper+, Schuster gave a detailed development of the analogy between the periodogram and the distribution of energy in a bright lined spectrum produced by a simple grating. The ordinate $S$ of the periodogram was there defined as $\frac{1}{4} R^{2} p n \alpha$, using the notation of the present chapter.

* Terrestrial Magnetism, Vol. III, p. 22.

† Camb. Phil. Soc. Trans., Vol. xviri, p. 107.

$\ddagger$ Proc. Roy. Soc., Vol. Lxxvir, p. 136. 
Schuster applied the method of the third paper to the discussion of the periodicities of sunspots*. In the course of this work the material accumulated during 150 years was used, and the periodogram was drawn for values of 'T' varying from 5.5 days to 24 years. The whole range of 150 years was then divided into two parts, of 75 years each, and the periodogram for each of these parts drawn separately. A very striking result was obtained. T'he two curves bore not the slightest resemblance to each other: During the first interval of 75 years the principal periods were $13 \frac{3}{4}$ years and $9 \frac{1}{4}$ years, while during the second interval, up to 1900 , the $11 \frac{1}{8}$ year period predominated. A number of other fairly clearly-defined periods were also discussed.

The whole discussion points to the conclusion that in such observational data as are generally treated by the periodogram method, we meet with a new type of periodicity which is illdefined, and not always persistent in amplitucle or phase. Outside the domain of gravitational astronomy one seldom finds isolated and clearly-defined periodicities. In the periodogram the problem is often complieated by the presence of not merely one or two well-marked periods, but of a large number of almost eoincident periods, so that the periodogram may then be compared to a spectrum full of closely-crowded lines. In such a case, a high resolving power is necessary in order to separate out the slightly differing periods; i.e. the observations used in the discussion must extend over as long a period as possible.

\section{Whittaker's variant of Schuster's method.}

In a discussion of the variations of SS Cygnit, Gibb applies a method suggested by Whittaker, the essential point of which is to defer the harmonic analysis until a late stage of the work. To examine the observed quantities $u_{0}, u_{1}, u_{2}$, ete. for a period of $p$ intervals we arrange them as before in $n$ rows of $p$ each, and add the columns, forming the sums $V_{0}, V_{1}, V_{2}$, ete. Then, instead of applying Fourier's analysis to the $V$ 's, we find the difference between the greatest and least of these quantities. This is a rough measure of the anplitude of the period of $p$ intervals.

* Phil. Trans. Roy. Soc., 206 A, pp. 69-100.

+ Monthly Notices, R.A.S., Vol. Lxxiv, p. 678. 
Let $y$ be the difference between the greatest and the least of the $V$ 's for a trial period $x$ (where $x=p x$ ). Draw a graph with $x$ as abscissa and $y$ as ordinate. The resulting curve may be called the "curve of periods." It shows high peaks at the points at which real periodicities exist, and lower peaks at points corresponding to doubtful periodicities. Such a curve of periods indicates the range of possible periods which can be usefully explored by a detailed periodogram analysis, and forms a useful preliminary to the application of Schuster's method.

\section{Use of the Complete Fourier Sequence.}

Turner* has suggested that the work of the periodogram might be more economically performed by the evaluation of the complete Fourier sequence of exact sub-multiples of the whole range of time covered by the observations. When the figures analysed form a regular series, with no sudden jumps between two consecutive values, it may be expected that the later coefficients in the Fourier sequence will be small. The observations can then be completely represented (with considerable accuracy) by a limited number of terms, and, theoretically at any rate, the Fourier sequence should contain within itself all the properties of the original observations, including the periodicities of those observations.

In the first place we must consider the effect of a period of the original observations whose length falls between two consecutive sub-multiples of the total range of time covered by the observations, and so falls between the periods represented by two consecutive terms of the Fourier sequence. It might be anticipated a priori that such a true period should affect most those terms in the Fourier sequence whose periods most nearly coincide with its own. This can easily be proved by simple calculation. Let the true period be represented by a single term, with unit amplitude

$$
\sin (p t+\delta)
$$

Let the resulting Fourier sequence be

$$
\sum_{q=0} A_{q} \cos q t+\sum_{q=1} B_{q} \sin q t .
$$

It will be assumed that the number of observations is sufficiently * Monthly Notices, R.A.S., Vol. Lxxir, pp. 549, 714. 
large to permit of our replacing summations by integrations. This simplifies the work considerably, and does not in any way affect the conclusions arrived at. We then have

$$
\begin{aligned}
\pi B_{q} & =\int_{0}^{2 \pi} \sin (p t+\delta) \sin q t d t \\
& =\frac{2 q}{p^{2}-q^{2}} \sin \pi p \cos (\pi p+\delta), \\
\pi A_{q} & =\int_{0}^{2 \pi} \sin (p t+\delta) \cos q t d t \\
& =\frac{2 p}{p^{2}-q^{2}} \sin \pi p \sin (\pi p+\delta) .
\end{aligned}
$$

If $p$ lies between $q$ and $q+1$, it is clear that $p^{2}-q^{2}$, and consequently $A$ and $B$, change sign as we go from $q$ to $q+1$. This change of sign affords a new criterion for evaluating periodicities, but is strictly valid only when the periodicity considered is isolated. Again the values of $A$ and $B$ will be greatest for those values of $q$ which yield the least values of $p^{2}-q^{2}$. Thus if there be an isolated periodicity between $q$ and $q+1$, the coefficients $A_{q}, A_{q+1}, B_{q}, B_{q+1}$ will be greater than any of the other coefficients in their neighbourhood.

Let

$$
p=q+x .
$$

Then

$$
\begin{aligned}
\pi A_{q} & =\frac{2 p}{p+q} \frac{\sin \pi x}{x} \cos (\pi x+\delta), \\
\pi A_{q+1} & =\frac{-2 p}{p+q+1} \frac{\sin \pi x}{1-x} \cos (\pi x+\delta),
\end{aligned}
$$

and

$$
\frac{A_{q}}{A_{q+1}}=-\frac{1-x}{x}=\frac{B_{q}}{B_{q+1}},
$$

when $q$ is large. Either of the two expressions will yield the value of $x$. This value is derived on the assumption that $q$ is large, but this condition will generally hold for any period worthy of serious consideration. If $R_{q}$ be the amplitude of the $q$ th period in the Fourier sequence,

$$
R_{q}^{2}=A_{q}^{2}+B_{q}^{2} \text { and } R_{q}=(-1)^{q} \frac{\sin \pi x}{\pi x}, \text { approximately. }
$$

This result may be compared with the equation $\frac{R}{R^{\prime}}=\frac{\sin \gamma}{\gamma}$ of $\S 90$. 
The following table, extracted from Turner's Tables for Harmonic Analysis, shows the effect of a periodic term of unit amplitude, for which $p=q+x$, on the coefficients of the neighbouring terms in the series.

\begin{tabular}{|c|c|c|c|c|c|c|}
\hline $\begin{array}{l}\text { Divisor } \\
\text { Factor }\end{array}$ & $\begin{array}{c}q-2 \\
+\frac{\sin \pi x}{\pi(x+2)}\end{array}$ & $\begin{array}{c}q-1 \\
+\frac{\sin \pi x}{\pi(x+1)}\end{array}$ & $\begin{array}{c}q \\
+\frac{\sin \pi x}{\pi x}\end{array}$ & $\begin{array}{c}q+1 \\
-\frac{\sin \pi x}{\pi(1-x)}\end{array}$ & $\begin{array}{c}q+2 \\
-\frac{\sin \pi x}{\pi(2-x)}\end{array}$ & $\begin{array}{c}q+3 \\
-\frac{\sin \pi x}{\pi(3-x)}\end{array}$ \\
\hline$x=0$ & .00 & $\cdot 00$ & $1 \cdot 00$ & .00 & $\cdot 00$ & $\cdot 00$ \\
\hline$x=\frac{1}{6}$ & .07 & $\cdot 14$ & $\cdot 95$ & $-\cdot 19$ & $-\cdot 09$ & $-\cdot 06$ \\
\hline$x=\frac{1}{3}$ & $\cdot 12$ & $\cdot 21$ & $\cdot 82$ & $-\cdot 41$ & $-\cdot 16$ & $-\cdot 10$ \\
\hline$x=\frac{1}{2}$ & $\cdot 13$ & $\cdot 21$ & 63 & $-\cdot 63$ & $-\cdot 21$ & $-\cdot 13$ \\
\hline$x=\frac{2}{3}$ & $\cdot 10$ & $\cdot 16$ & $\cdot 41$ & -.82 & $-\cdot 21$ & $-\cdot 12$ \\
\hline$x=\frac{5}{6}$ & .06 & $\cdot 09$ & $\cdot 19$ & -.95 & $-\cdot 14$ & $-\cdot 07$ \\
\hline$x=1$ & $\cdot 00$ & .00 & $\cdot 00$ & $-1 \cdot 00$ & $\cdot 00$ & .00 \\
\hline
\end{tabular}

This table gives the value of the factor $\frac{\sin \pi p}{\pi(p-q)}$ in the values for $A_{q}$ and $B_{q}$ given above, the factor $\frac{2 p}{p+q}$ or $\frac{-q}{p+q}$ being treated as unity.

It is clear from the table that a single periodicity can only yield large values to a few coefficients in the Fourier series. This agrees with the hump in the periodogram which occurs in the neighbourhood of a true period. Thus the Fourier series ought in theory to yield the values of the true periodicities with less work than Schuster's method demands. For the occurrence of two or three large coefficients in the Fourier sequence indicates the presence of a true period between the two terms which have the largest coefficients, and the equation

$$
\frac{1-x}{x}=-\frac{A_{q}}{A_{q+1}}=-\frac{B_{q}}{B_{q+1}}
$$

should give the accurate value of $x$, replacing the secondary analysis of Schuster's method. 
In practice, however, the matter is by no means so simple, as may be seen by considering the following extract from 'Turner's table of the Fourier sequence for Wolf's sunspot numbers (156 years).

\begin{tabular}{|c|c|c|c|c|}
\hline Divisor & Period & d & $B$ & $R$ \\
\hline 12 & 13.0 years & $4 \cdot 8$ & $5 \cdot 9$ & $7 \cdot 6$ \\
\hline 13 & $12 \cdot 0$ & $3 \cdot 1$ & $14 \cdot 7$ & $15 \cdot 0$ \\
\hline 14 & $11 \cdot 14$ & $15 \cdot 8$ & $-19 \cdot 6$ & $25 \cdot 8$ \\
\hline 15 & $10 \cdot 40$ & $-4 \cdot 8$ & $-13 \cdot 2$ & $14 \cdot 0$ \\
\hline 16 & $9 \cdot 75$ & $13 \cdot 6$ & $0 \cdot 4$ & $13 \cdot 6$ \\
\hline 17 & $9 \cdot 20$ & $3 \cdot 7$ & $6 \cdot 5$ & $7 \cdot 5$ \\
\hline
\end{tabular}

The largest value of $R$ is at $q=14$, showing the existence of a true period near $11 \cdot 14$ years, near the supposedly well-known 11 year period. According to the theory given above there should be a change of sign of both $A$ and $B$ on one side of $q=14$, but not on the other. But the table shows a change of sign of $A$ from $q=14$ to $q=15$, and of $B$ from $q=13$ to $q=14$. It is thus probable that more than one real periodicity falls within the range of the table given above.

When the changes of sign of $A$ and $B$ agree, it will generally be found that the values of $x$ deduced from the $A$ 's and the $B$ 's differ. In such a case it is perhaps better to use the equation

$$
-\frac{1-x}{x}=\begin{gathered}
A_{q} \pm B_{q} \\
A_{q+1} \pm B_{q+1}
\end{gathered}
$$

Take, for example, the following extract from the Fourier sequence for the Greenwich temperatures:

\begin{tabular}{|r|r|r|r|}
\hline$q$ & \multicolumn{1}{|c|}{$A$} & \multicolumn{1}{|c|}{$B$} & $R$ \\
\hline 22 & $\cdot 4$ & $1 \cdot 0$ & $1 \cdot 0$ \\
23 & -3.0 & 4.3 & $5 \cdot 2$ \\
24 & $\cdot$ & $-2 \cdot 8$ & 2.9 \\
\hline
\end{tabular}


Putting

we find

therefore

$$
-\frac{1-x}{x}=\frac{7 \cdot 3}{3 \cdot 5}
$$$$
x=\frac{3 \cdot 5}{10 \cdot 8}=\cdot 324
$$$$
p=23 \cdot 324 \text {. }
$$

When a large number of consecutive terms of the Fourier sequence are large, or the law of signs of $A$ and $B$ is not followed, it is generally safe to conclude that a number of periodicities are involved. In such a case it is best to examine the observations for possible discontinuities.

\section{The Investigation of Discontinuities*.}

In order to investigate the possible discontinuities of the 11 year sunspot period, Turner arranged the sunspot numbers in periods of 12 years. The coefficients $A, B$ were evaluated for years 1 to 12. Then the year 13 was substituted for the year 1 , and the coefficients $A, B$ were re-calculated. Next the year 14 was substituted for the year 2 ; and the process was continued until the whole series of sunspot numbers had been used up. The work can be carried out in the following way. In Table I are given the Wolf sunspot numbers arranged in periods of 12 years. In Table II the first row is identical with the first row in Table I, the second row is the difference of row 2 and row 1 of Table $I$, etc.

\section{TABLE I.}

Year

\begin{tabular}{rrrrrrrrrrrrr}
1749 & 81 & 83 & 48 & 48 & 31 & 12 & 10 & 10 & 32 & 48 & 54 & 63 \\
1761 & 86 & 61 & 45 & 36 & 21 & 11 & 38 & 70 & 106 & 101 & 82 & 66 \\
1773 & 35 & 31 & 7 & 20 & 92 & 154 & 126 & 85 & 68 & 38 & 23 & 10 \\
etc. & & \multicolumn{3}{c}{ etc. } & & & & & & &
\end{tabular}

\section{TABLE II.}

\begin{tabular}{rrrrrrrrrrrrr}
1749 & 81 & 83 & 48 & 48 & 31 & 12 & 10 & 10 & 32 & 48 & 54 & 63 \\
1761 & 5 & -22 & -3 & -12 & -10 & -1 & 28 & 60 & 74 & 53 & 28 & 3 \\
1773 & -51 & -30 & -38 & -16 & $\begin{array}{c}71 \\
\text { etc. }\end{array}$ & 143 & 88 & 15 & -38 & -63 & -59 & -56 \\
etc. & & \multicolumn{3}{c}{ etc. }
\end{tabular}

TABLE III.

Products for cosines.

$\begin{array}{rrrrrrrrrrrrr}1749 & 81 & 72 & 24 & 0 & -16 & -10 & -10 & -9 & -16 & 0 & 27 & 55 \\ 1761 & 5 & -19 & -2 & 0 & 5 & 1 & -28 & -52 & -37 & 0 & 14 & 3 \\ 1773 & -51 & -26 & -19 & 0 & -36 & -124 & -88 & -13 & 19 & 0 & -30 & -48\end{array}$

* Turner, Nonthly Notices, R.A.S., Vol. Lxxiv, p. 82. 
'T'ABLE IV.

Products for sines.

$\begin{array}{rrrrrrrrrrrrr}\text { Year } & & & & & & & & & \\ 1749 & 0 & 42 & 41 & 48 & 27 & 6 & 0 & -5 & -28 & -48 & -47 & -32 \\ 1761 & 0 & -11 & -3 & -12 & -9 & 0 & 0 & -30 & -65 & -53 & -24 & -2 \\ 1773 & 0 & -15 & -33 & -16 & 62 & 72 & 0 & -8 & 33 & 63 & 51 & 28\end{array}$

Table III is derived by multiplying the columns of 'Table II by the appropriate factors $\cos 0^{\circ}, \cos 30^{\circ}, \cos 60^{\circ}$, etc. 'The factors are $1, \cdot 866, \cdot 5,0,-\cdot 5,-866,-1,-\cdot 866,-\cdot 5,0, \cdot 5, \cdot 866$. Similarly Table IV is derived by multiplying the columns of Table II by the appropriate factors $\sin 0^{\circ}, \sin 30^{\circ}, \sin 60^{\circ}$, etc. These factors are $0, \cdot 5, \cdot 866,1, \cdot 866, \cdot 5,0,-\cdot 5,-\cdot 866,-1,-\cdot 866,-\cdot 5$.

The difference between $A, B$ for the 12 year interval starting at 1749 , and the interval starting at 1750 , is due to the substitution of 86 for 81 in the first term. The difference, 5, is given in Table II, and is multiplied by the appropriate factors, 1 and 0 , in Tables III and IV. To find $A, B$, for the 12 year interval starting at 1751 , we must substitute 61 for 83 in the second term. This is the difference of -22 given in Table II, and multiplied by the appropriate factors in Tables III and IV. The process of evaluation of successive $A$ 's and $B$ 's is thus reduced to the addition of successive terins of Tables III and IV.

Thus we obtain a series of values of $A$ and $B$. The phase $\phi$ is deduced from the formula

$$
\tan \phi=\frac{B}{A} .
$$

From the progression of the values of $\phi$ the accurate period can be evaluated as from fig. 13. In the work on the sunspot numbers, Turner found that the progression of phase underwent sudden changes near the years $1766,1796,1838,1868$, and 1895, while in the intervals between these breaks the progression of phase was regular. Thus from 1749 to 1766 the phase could be represented by a formula

$$
\phi=\text { const. }-3^{\circ} . t
$$

where $t$ is measured in years. This corresponds to a period of

$$
\frac{12}{1+\frac{36}{360}} \text { years }=10.9 \text { years. }
$$

B. 0 . 
Again from about 1760 to 1796 the phase could be represented with fair accuracy by a formula

$$
\phi=\text { const. }-8 \cdot 3^{\circ} \cdot t \text {, }
$$

corresponding to a period of

$$
\frac{12}{1+\frac{12 \times 8 \times 3}{360}} \text { years, or } 9 \cdot 37 \text { years. }
$$

Turner's results show clearly that the predominating periodicity changes at intervals, bearing out the results previously derived by Schuster.

Turner's method of investigating discontinuities in the main periodicities is to be recommended in all periodogram work. It affords the surest method of detecting changes in the relative importance of different periodicities from time to time. The labour involved is not prohibitive, particularly when it is considered in conjunction with the importance of its results.

\section{An Application of the Fourier Sequence in Periodogram Work.}

The following brief details of an analysis' of the Greenwich Temperature records undertaken by the present writer, but not yet completed, may help to illustrate the method of using the complete Fourier sequence. The records used in the first analysis extended from 1841 to 1890 , and the monthly mean temperatures were used. The effect of the annual period and all its submultiples was removed from the records by subtracting from each monthly mean the mean temperature of the corresponding month during the whole interval of 50 years. These quantities subtracted were analysed separately (see Ex. 3, page 185). In order to avoid negative signs as far as possible, $10^{\circ}$ was added to the figures obtained after subtracting the general mean for the whole period from the monthly means. The figures so obtained, expressed in units of $\cdot 1^{\circ}$, were taken as the material for the periodogram analysis. There were $12 \times 50=600$ figures.

Adding together the figures for 25 consecutive months, and dividing by 25 , we obtain 24 terms, each of which is the mean deviation for 25 months. These figures were analysed by the usual method up to the 4th harmonic (see Ex. 1, p. 188). Next, 
the figures for 10 consecutive months were ardded, yiclding 60 terms, and these wereanalysed for the 5 th, 6 th,... up to the 14 th harmonies. The first 14 periods of the Fourier sequence were thus obtained, except that they needed a correction of phase, the two sets of terms being determined with regard to different origins of time.

Certain sets of coefficients were capable of fairly easy evaluation. Thus the period of 40 months and its submultiples were obtained by writing down in a table the 15 periods of 40 months, and taking the means of the columns. The analysis of these means yielded the terms $q=15,30,45,60$, etc. in the Fourier sequence.

'The terms $q=20,40,60$, etc., and the terms $q=25$, 75, etc., were also evaluated in a similar manner. The remainder of the terms up to $q=49$ were calculated by a straightforward application of the formulae of $\$ \$ 8,86$, though this involved considerable labour.

The resulting Fourier sequence shows a number of fairly clearly marked periodicities, but a closer examination reveals evidences of discontinuities.

The figures obtained when the general mean for 50 years for any one month is subtracted from the corresponding monthly means, do not form a regular series as do the sunspot numbers. It cannot be expected that the coefficients of the later harmonics in the series will become vanishingly small. The apparent chance distribution of the figures analysed will tend to give fairly large values of the later coefficients even when there are no real periodicities in that region. In other words, the mean value of $R^{2}$ will be large in regions where no real periodicity exists, and so it will be necessary to consider very carefully whether the apparent periods obtained may not be due to a chance distribution of the figures analysed. 


\section{APPENDIX I}

\section{LIST OF REFERENCES}

The following short list of references, supplementing those given in the text, may prove of use to the student who desires to read original memoirs bearing upon different parts of the subject.

\section{CHAPTER II}

Bernsteix. "On Gauss's Error Law." Math. Annalen, Vol. Lxiv, p. 417. GLAIsher. "On the Law of Facility of Errors of Observations, and on the Method of Least Squares." Mem. R.A.S., Vol. xxxix. (A general discussion of a number of different proofs of Gauss's Law of Errors.)

Searle. "Geometrical Methods of Combining Observations." Lick Observatory Bulletin, Vol. Lx.

Storx. "The Law of Error developed from a new Standpoint." Proc. Amer. Acad. Arts and Sci., Vol. Lx, p. 167.

Full Tables of $\frac{2}{\sqrt{\pi}} \int_{0}^{t} e^{-t^{2}} d t$. Trans. Royal Soc. Edin., Vol. xxxıx, p. 257.

\section{CHAPTER VI}

Glatsher. "Determinant Method of Solution of the Normal Equations." Monthly Notices, R.A.S., Vol. xL, p. 600; Vol. xLI, p. 18.

"Solution of the Normal Equations by Gauss's Method." Monthly Notices, R.A.S., Vol. xxxiv, p. 311.

\section{CHAPTER IX}

Pearsow, K. "Skew Variation in Homogeneous Material." Phil. Trans. R.S., Vol. 186 A, p. 343 ; Vol. 197 A, p. 443.

"On the Systematic Fitting of Curres to Observations and Measurements." Biometrika, Vol. I, p. 265 ; Vol. II, p. 1.

"On the Curves which are most suitable for describing the Frequency of Random Samples of a Population." Biometrika, Vol. v, p. 172. 


\section{CHAP'TER $\mathrm{X}$}

Brows, W. The Essentials of Mentul Measurement. Cimblridge, 1911. (Applications of Correlation in Psychology.)

Burr, C. "Experimental Tests of General Intelligence." Brit. Jour. Paych., Vol. III, p. 94.

Eudnton, W. P. Frequency Curves and Correlation. (Deals largely with actuarial applications.)

Pearson, K. "Regression, Heredity, and Panminia." Phil. Truns. R.S., Vol. $187 \wedge$, p. 253.

"On the Theory of Contingency and its Relation to Association and Normal Correlation." Drapers' Co. Research Memoirs, Biometric Series I, 1904.

"On the Theory of Skew-Correlation and Non-Linear Regression." Diapers' Co. Researeh Memoirs, Biometric Series II, 1905.

"On Further Methods of Determining Correlation." Drapers' Co. Research Memoirs, Biometric Series Iv, 1907.

Yule, G. U. An Introduction to the Theory of Statisties. 1912. 


\section{APPENDIX II}

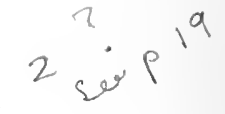

TABLE OF $\Theta(t)=\frac{2}{\sqrt{\pi}} \int_{0}^{t} e^{-t} d t$.

\begin{tabular}{|c|c|c|c|c|c|}
\hline$t$ & $\boldsymbol{\theta}(t)$ & Diff. & $t$ & $\theta(t)$ & Diff. \\
\hline $\begin{array}{c}0 \cdot 00 \\
\cdot 01 \\
\cdot 02 \\
\cdot 03 \\
\cdot 04\end{array}$ & $\begin{array}{r}0.00000 \\
\cdot 01128 \\
\cdot 02256 \\
\cdot 03384 \\
\cdot 04511\end{array}$ & $\begin{array}{l}1128 \\
1128 \\
1128 \\
1127 \\
1126\end{array}$ & $\begin{array}{c}0 \cdot 30 \\
\cdot 31 \\
\cdot 32 \\
\cdot 33 \\
\cdot 34\end{array}$ & $\begin{array}{r}0 \cdot 32863 \\
\cdot 33891 \\
\cdot 34913 \\
\cdot 35928 \\
\cdot 36936\end{array}$ & $\begin{array}{l}1028 \\
1022 \\
1015 \\
1008 \\
1002\end{array}$ \\
\hline $\begin{array}{r}0 \cdot 05 \\
\cdot 06 \\
.07 \\
.08 \\
.09\end{array}$ & $\begin{array}{r}0 \cdot 05637 \\
\cdot 06762 \\
\cdot 07886 \\
\cdot 09008 \\
\cdot 10128\end{array}$ & $\begin{array}{l}1125 \\
1124 \\
1122 \\
1120 \\
1118\end{array}$ & $\begin{array}{r}0 \cdot 35 \\
\cdot 36 \\
\cdot 37 \\
\cdot 38 \\
\cdot 39\end{array}$ & $\begin{array}{r}0 \cdot 37938 \\
\cdot 38933 \\
\cdot 39921 \\
\cdot 40901 \\
\cdot 41874\end{array}$ & $\begin{array}{l}995 \\
988 \\
980 \\
973 \\
965\end{array}$ \\
\hline $\begin{array}{r}0 \cdot 10 \\
\cdot 11 \\
\cdot 12 \\
\cdot 13 \\
\cdot 14\end{array}$ & $\begin{array}{r}0 \cdot 11246 \\
\cdot 12362 \\
\cdot 13476 \\
\cdot 14587 \\
\cdot 15695\end{array}$ & $\begin{array}{l}1116 \\
1114 \\
1111 \\
1108 \\
1105\end{array}$ & $\begin{array}{r}0 \cdot 40 \\
\cdot 41 \\
\cdot 42 \\
\cdot 43 \\
\cdot 44\end{array}$ & $\begin{array}{r}0.42839 \\
\cdot 43797 \\
\cdot 44747 \\
\cdot 45689 \\
\cdot 46623\end{array}$ & $\begin{array}{l}958 \\
950 \\
942 \\
934 \\
925\end{array}$ \\
\hline $\begin{array}{r}0 \cdot 15 \\
\cdot 16 \\
\cdot 17 \\
\cdot 18 \\
\cdot 19\end{array}$ & $\begin{array}{r}0 \cdot 16800 \\
\cdot 17901 \\
\cdot 18999 \\
\cdot 20094 \\
\cdot \cdot 21184\end{array}$ & $\begin{array}{l}1101 \\
1098 \\
1095 \\
1090 \\
1086\end{array}$ & $\begin{array}{r}0 \cdot 45 \\
\cdot 46 \\
\cdot 47 \\
\cdot 48 \\
\cdot 49\end{array}$ & $\begin{array}{r}0.47548 \\
\cdot 48466 \\
.49375 \\
.50275 \\
.51167\end{array}$ & $\begin{array}{l}918 \\
909 \\
900 \\
892 \\
883\end{array}$ \\
\hline $\begin{array}{r}0 \cdot 20 \\
\cdot 21 \\
\cdot 22 \\
\cdot 23 \\
\cdot 24\end{array}$ & $\begin{array}{r}0 \cdot 22270 \\
\cdot 23352 \\
\cdot 24430 \\
\cdot 25502 \\
\cdot 26570\end{array}$ & $\begin{array}{l}1082 \\
1078 \\
1072 \\
1068 \\
1063\end{array}$ & $\begin{array}{r}0.50 \\
.51 \\
.52 \\
.53 \\
.54\end{array}$ & $\begin{array}{r}0.52050 \\
.52924 \\
.53790 \\
.54646 \\
.55494\end{array}$ & $\begin{array}{l}874 \\
866 \\
856 \\
848 \\
838\end{array}$ \\
\hline $\begin{array}{r}0 \cdot 25 \\
\cdot 26 \\
\cdot 27 \\
\cdot 28 \\
\cdot 29\end{array}$ & $\begin{array}{r}0 \cdot 27633 \\
\cdot 28690 \\
\cdot 29742 \\
\cdot 30788 \\
\cdot 31828\end{array}$ & $\begin{array}{l}1057 \\
1052 \\
1046 \\
1040 \\
1035\end{array}$ & $\begin{array}{r}0.55 \\
.56 \\
.57 \\
.58 \\
.59\end{array}$ & $\begin{array}{r}0.56332 \\
.57162 \\
.57982 \\
.58792 \\
.59594\end{array}$ & $\begin{array}{l}830 \\
820 \\
810 \\
802 \\
792\end{array}$ \\
\hline $0 \cdot 30$ & $0 \cdot 32863$ & . & $0 \cdot 60$ & 0.60386 & - \\
\hline
\end{tabular}




\begin{tabular}{|c|c|c|c|c|c|}
\hline$t$ & $\theta(t)$ & I)ifl. & $t$ & $\theta(t)$ & Dilf. \\
\hline $\begin{array}{r}0 \cdot 60 \\
\cdot 61 \\
\cdot 62 \\
\cdot 63 \\
\cdot 64\end{array}$ & $\begin{array}{r}0 \cdot 60386 \\
\cdot 61168 \\
\cdot 61941 \\
\cdot 62705 \\
\cdot 63459\end{array}$ & $\begin{array}{l}782 \\
773 \\
764 \\
754 \\
744\end{array}$ & $\begin{array}{r}1 \cdot 00 \\
\cdot 01 \\
\cdot 02 \\
\cdot 03 \\
\cdot 04\end{array}$ & $\begin{array}{r}0.84270 \\
.84681 \\
.85084 \\
.85478 \\
.85865\end{array}$ & $\begin{array}{l}411 \\
403 \\
394 \\
387 \\
379\end{array}$ \\
\hline $\begin{array}{r}0 \cdot 65 \\
\cdot 66 \\
\cdot 67 \\
\cdot 68 \\
\cdot 69\end{array}$ & $\begin{array}{r}0 \cdot 64203 \\
\cdot 64938 \\
\cdot 6.3663 \\
\cdot 66: 378 \\
\cdot 67084\end{array}$ & $\begin{array}{l}735 \\
725 \\
715 \\
706 \\
696\end{array}$ & $\begin{array}{r}1 \cdot 05 \\
.06 \\
\cdot 07 \\
.08 \\
.09\end{array}$ & $\begin{array}{r}0.86244 \\
.86614 \\
.86977 \\
.87333 \\
.87680\end{array}$ & $\begin{array}{l}370 \\
363 \\
356 \\
347 \\
341\end{array}$ \\
\hline $\begin{array}{r}0 \cdot 70 \\
\cdot 71 \\
\cdot 72 \\
\cdot 73 \\
.74\end{array}$ & $\begin{array}{r}0 \cdot 67780 \\
\cdot 68467 \\
\cdot 69143 \\
\cdot 69810 \\
\cdot 70468\end{array}$ & $\begin{array}{l}687 \\
676 \\
667 \\
658 \\
648\end{array}$ & $\begin{array}{c}1 \cdot 10 \\
\cdot 11 \\
\cdot \cdot 12 \\
\cdot 13 \\
\cdot 14\end{array}$ & $\begin{array}{r}0.88021 \\
.88353 \\
.88679 \\
.88997 \\
.89308\end{array}$ & $\begin{array}{l}332 \\
326 \\
318 \\
311 \\
304\end{array}$ \\
\hline $\begin{array}{r}0 \cdot 75 \\
\cdot 76 \\
\cdot 77 \\
\cdot 78 \\
\cdot 79\end{array}$ & $\begin{array}{r}0 \cdot 71116 \\
\cdot 71754 \\
772382 \\
\cdot 73001 \\
\cdot 73610\end{array}$ & $\begin{array}{l}638 \\
628 \\
619 \\
609 \\
600\end{array}$ & $\begin{array}{r}1 \cdot 15 \\
\cdot 16 \\
\cdot 17 \\
\cdot 18 \\
\cdot 19\end{array}$ & $\begin{array}{r}0.89612 \\
.89910 \\
.90200 \\
.90484 \\
.90761\end{array}$ & $\begin{array}{l}298 \\
290 \\
284 \\
277 \\
270\end{array}$ \\
\hline $\begin{array}{r}0.80 \\
.81 \\
.82 \\
.83 \\
.84\end{array}$ & $\begin{array}{r}0.74210 \\
774800 \\
775381 \\
775952 \\
\cdot 76514\end{array}$ & $\begin{array}{l}590 \\
581 \\
571 \\
562 \\
553\end{array}$ & $\begin{array}{r}1 \cdot 20 \\
\cdot 21 \\
\cdot 22 \\
\cdot 23 \\
\cdot 24\end{array}$ & $\begin{array}{r}0.91031 \\
.91296 \\
.91553 \\
.91805 \\
.92051\end{array}$ & $\begin{array}{l}265 \\
257 \\
252 \\
246 \\
239\end{array}$ \\
\hline $\begin{array}{r}0 \cdot 85 \\
.86 \\
.87 \\
.88 \\
.89\end{array}$ & $\begin{array}{r}0 \cdot 77067 \\
\cdot 77610 \\
\cdot 78144 \\
\cdot 78669 \\
\cdot 79184\end{array}$ & $\begin{array}{l}543 \\
534 \\
525 \\
515 \\
507\end{array}$ & $\begin{array}{r}1 \cdot 25 \\
\cdot 26 \\
\cdot 27 \\
\cdot 28 \\
\cdot 29\end{array}$ & $\begin{array}{r}0.92290 \\
.92524 \\
.92751 \\
.92973 \\
.93190\end{array}$ & $\begin{array}{l}234 \\
227 \\
222 \\
217 \\
211\end{array}$ \\
\hline $\begin{array}{r}0.90 \\
.91 \\
.92 \\
\cdot 93 \\
\cdot 94\end{array}$ & $\begin{array}{r}0.79691 \\
.80188 \\
.80677 \\
.81156 \\
\cdot 81627\end{array}$ & $\begin{array}{l}497 \\
489 \\
479 \\
471 \\
462\end{array}$ & $\begin{array}{r}1 \cdot 30 \\
\cdot 31 \\
\cdot 32 \\
\cdot 33 \\
\cdot 34\end{array}$ & $\begin{array}{r}0.93401 \\
\cdot 93606 \\
\cdot 93807 \\
\cdot 94002 \\
\cdot 94191\end{array}$ & $\begin{array}{l}205 \\
201 \\
195 \\
189 \\
185\end{array}$ \\
\hline $\begin{array}{r}0 \cdot 95 \\
\cdot 96 \\
\cdot 97 \\
\cdot 98 \\
\cdot 99\end{array}$ & $\begin{array}{r}0.82089 \\
.82542 \\
.82987 \\
.83423 \\
.83851\end{array}$ & $\begin{array}{l}453 \\
445 \\
436 \\
428 \\
419\end{array}$ & $\begin{array}{r}1 \cdot 35 \\
\cdot 36 \\
\cdot 37 \\
\cdot 38 \\
\cdot 39\end{array}$ & $\begin{array}{r}0.94376 \\
.94556 \\
.94731 \\
\cdot .94902 \\
.95067\end{array}$ & $\begin{array}{l}180 \\
175 \\
171 \\
165 \\
162\end{array}$ \\
\hline $1 \cdot 00$ & $0 \cdot 84270$ & & $1 \cdot 40$ & 0.95229 & \\
\hline
\end{tabular}




\begin{tabular}{|c|c|c|c|c|c|}
\hline$t$ & $\Theta(t)$ & Diff. & $t$ & $\theta(t)$ & Diff. \\
\hline $\begin{array}{r}1 \cdot 40 \\
\cdot 41 \\
\cdot 42 \\
\cdot 43 \\
\cdot 44\end{array}$ & $\begin{array}{r}0.95229 \\
.95385 \\
.95538 \\
.95686 \\
.95830\end{array}$ & $\begin{array}{l}156 \\
153 \\
148 \\
144 \\
140\end{array}$ & $\begin{array}{r}1 \cdot 80 \\
\cdot 81 \\
\cdot 82 \\
\cdot 83 \\
\cdot 84\end{array}$ & $\begin{array}{r}0 \cdot 98909 \\
\cdot 98952 \\
\cdot 98994 \\
\cdot 99035 \\
\cdot 99074\end{array}$ & $\begin{array}{l}43 \\
42 \\
41 \\
39 \\
37\end{array}$ \\
\hline $\begin{array}{r}1 \cdot 45 \\
\cdot 46 \\
\cdot 47 \\
\cdot 48 \\
\cdot 49\end{array}$ & $\begin{array}{r}0.95970 \\
.96105 \\
.96237 \\
.96365 \\
.96490\end{array}$ & $\begin{array}{l}135 \\
132 \\
128 \\
125 \\
121\end{array}$ & $\begin{array}{r}1 \cdot 85 \\
.86 \\
.87 \\
.88 \\
.89\end{array}$ & $\begin{array}{r}0.99111 \\
.99147 \\
.99182 \\
.99216 \\
.99248\end{array}$ & $\begin{array}{l}36 \\
35 \\
34 \\
32 \\
31\end{array}$ \\
\hline $\begin{array}{r}1.50 \\
.51 \\
.52 \\
.53 \\
.54\end{array}$ & $\begin{array}{r}0.96611 \\
.96728 \\
.96841 \\
.96952 \\
.97059\end{array}$ & $\begin{array}{l}117 \\
113 \\
111 \\
107 \\
103\end{array}$ & $\begin{array}{r}1 \cdot 90 \\
\cdot 91 \\
\cdot 92 \\
\cdot 93 \\
\cdot 94\end{array}$ & $\begin{array}{r}0.99279 \\
.99309 \\
.99338 \\
.99366 \\
.99392\end{array}$ & $\begin{array}{l}30 \\
29 \\
28 \\
26 \\
26\end{array}$ \\
\hline $\begin{array}{r}1 \cdot 55 \\
.56 \\
.57 \\
.58 \\
.59\end{array}$ & $\begin{array}{r}0 \cdot 97162 \\
.97263 \\
.97360 \\
.97455 \\
.97546\end{array}$ & $\begin{array}{r}101 \\
97 \\
95 \\
91 \\
89\end{array}$ & $\begin{array}{r}1 \cdot 9.5 \\
\cdot 96 \\
\cdot 97 \\
\cdot 98 \\
\cdot 99\end{array}$ & $\begin{array}{r}0.99418 \\
.99443 \\
.99466 \\
.99489 \\
\cdot 99511\end{array}$ & $\begin{array}{l}25 \\
23 \\
23 \\
22 \\
21\end{array}$ \\
\hline $\begin{array}{r}1 \cdot 60 \\
\cdot 61 \\
\cdot 62 \\
\cdot 63 \\
\cdot 64\end{array}$ & $\begin{array}{r}0.97635 \\
\cdot 97721 \\
.97804 \\
.97884 \\
.97962\end{array}$ & $\begin{array}{l}86 \\
83 \\
80 \\
78 \\
76\end{array}$ & $\begin{array}{c}2 \cdot 00 \\
\cdot 01 \\
\cdot 02 \\
\cdot 03 \\
\cdot 04\end{array}$ & $\begin{array}{r}0.99532 \\
.99552 \\
.99572 \\
.99591 \\
.99609\end{array}$ & $\begin{array}{l}20 \\
20 \\
19 \\
18 \\
17\end{array}$ \\
\hline $\begin{array}{r}1 \cdot 65 \\
\cdot 66 \\
\cdot 67 \\
\cdot 68 \\
\cdot 69\end{array}$ & $\begin{array}{r}0 \cdot 98038 \\
\cdot 98110 \\
\cdot 98181 \\
\cdot 98249 \\
\cdot 98315\end{array}$ & $\begin{array}{l}72 \\
71 \\
68 \\
66 \\
64\end{array}$ & $\begin{array}{c}2 \cdot 05 \\
\cdot 06 \\
\cdot 07 \\
\cdot 08 \\
\cdot 09\end{array}$ & $\begin{array}{r}0 \cdot 99626 \\
\cdot 99642 \\
.99658 \\
\cdot 99673 \\
\cdot 99688\end{array}$ & $\begin{array}{l}16 \\
16 \\
15 \\
15 \\
14\end{array}$ \\
\hline $\begin{array}{r}1 \cdot 70 \\
\cdot 71 \\
\cdot 72 \\
\cdot 73 \\
\cdot 74\end{array}$ & $\begin{array}{r}0.98379 \\
\cdot 98441 \\
\cdot 98500 \\
.98558 \\
\cdot 98613\end{array}$ & $\begin{array}{l}62 \\
59 \\
58 \\
55 \\
54\end{array}$ & $\begin{array}{r}2 \cdot 10 \\
\cdot 11 \\
\cdot 12 \\
\cdot 13 \\
\cdot 14\end{array}$ & $\begin{array}{r}0.99702 \\
.99715 \\
.99728 \\
.99741 \\
.99753\end{array}$ & $\begin{array}{l}13 \\
13 \\
13 \\
12 \\
11\end{array}$ \\
\hline $\begin{array}{r}1 \cdot 75 \\
\cdot 76 \\
\cdot 77 \\
\cdot 78 \\
\cdot 79\end{array}$ & $\begin{array}{r}0.98667 \\
\cdot 98719 \\
.98769 \\
.98817 \\
\cdot 98864\end{array}$ & $\begin{array}{l}52 \\
50 \\
48 \\
47 \\
45\end{array}$ & $\begin{array}{r}2 \cdot 15 \\
\cdot 16 \\
\cdot 17 \\
\cdot 18 \\
\cdot 19\end{array}$ & $\begin{array}{r}0.99764 \\
.99775 \\
.99785 \\
.99795 \\
.99805\end{array}$ & $\begin{array}{r}11 \\
10 \\
10 \\
10 \\
9\end{array}$ \\
\hline $1 \cdot 80$ & 0.98909 & & $2 \cdot 20$ & $0 \cdot 99814$ & 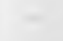 \\
\hline
\end{tabular}




\begin{tabular}{|c|c|c|c|}
\hline$t$ & $\theta(t)$ & $t$ & $\theta(t)$ \\
\hline $2 \cdot 20$ & $(0 \cdot 99814$ & $2 \cdot(; 0)$ & 1).99976 \\
\hline$\cdot 21$ & $998 \div 2$ & . 6.5 & 99982 \\
\hline 22 & $· 99831$ & 70 & -99987 \\
\hline 93 & $\cdot 99839$ & 75 & •99990 \\
\hline$\cdot 24$ & $\cdot 99846$ & $\cdot 80$ & •9999:3 \\
\hline $2 \cdot 2.7$ & 0.99854 & $2 \cdot 85$ & $0.99994 \quad 4$ \\
\hline$\cdot 26$ & •99861 & $\cdot 90$ & (9999:3 9 \\
\hline$\cdot 27$ & •99867 & ו) & 999970 \\
\hline$\cdot 28$ & 99874 & $3 \cdot 00$ & 8999 \\
\hline$\cdot 29$ & $• 99880$ & •0.5 & $\cdot 99998 \quad 4$ \\
\hline $2 \cdot 30$ & $0 \cdot 99886$ & $3 \cdot 10$ & $0.99998 \quad 84$ \\
\hline$\cdot 31$ & $• 99891$ & $\cdot 1 \overline{1}$ & •99999 16 \\
\hline$: 32$ & .99897 & $\cdot 20$ & •99999 40 \\
\hline 33 & $\cdot 9990 \cdot 2$ & $\cdot 25$ & 79999 \\
\hline$\cdot 34$ & $\bullet 99906$ & $\cdot 30$ & •99999 69 \\
\hline $2 \cdot 35$ & 0.99911 & $3 \cdot 35$ & 0.9999978 \\
\hline 36 & •99915 & $\cdot 40$ & •99999 85 \\
\hline $3 \overline{1}$ & •99920 & $\cdot 45$ & •99999 89 \\
\hline 38 & $\bullet 99924$ & $\cdot 50$ & •99999993 \\
\hline 39 & •99928 & 55 & •99999) 95 \\
\hline $2 \cdot 40$ & $0 \cdot 99931$ & $3 \cdot 60$ & $0 \cdot 99999 \quad 96441$ \\
\hline$\cdot 41$ & .99935 & .70 & .9999998329 \\
\hline 42 & $\cdot 99938$ & 80 & •99999 99230 \\
\hline$-4: 3$ & $\bullet 99941$ & $\cdot 90$ & •99999999652 \\
\hline$\cdot 44$ & $: 99944$ & $4 \cdot 00$ & •99999 99845 \\
\hline $2 \cdot 45$ & $0 \cdot 99947$ & $4 \cdot 10$ & $0 \cdot 99999 \quad 99933$ \\
\hline$\cdot 46$ & •99950 & -20 & וד999 99999י \\
\hline$\cdot 47$ & '99952 & $\cdot 30$ & ·99999 99988 \\
\hline$\cdot 48$ & •9995:) & $\cdot 40$ & •99999 99995 \\
\hline$\cdot 49$ & •99957 & ") & •99999 99998 \\
\hline $2 \cdot 50$ & $0 \cdot 99959$ & $4 \cdot 60$ & 0.99999999992 \\
\hline 51 & $• 99961$ & .70 & •99999 $99999 \quad$ † \\
\hline .52 & -99963 & $\cdot 80$ & •99999 999999 \\
\hline 53 & $\bullet 99965$ & $\cdot 90$ & כ) 99999 99999 959 \\
\hline 54 & 99967 & $5 \cdot 00$ & $1-1 \cdot \tilde{a} \times 10^{-12}$ \\
\hline $2 \cdot 55$ & $0 \cdot 99969$ & $5 \cdot 50$ & $1-7 \times 10^{-1 i}$ \\
\hline$• 56$ & $\cdot 99971$ & $6 \cdot 00$ & $1-2 \cdot 2 \times 10^{-1 i}$ \\
\hline 57 & $\cdot 99972$ & & \\
\hline 58 & 99974 & & \\
\hline$\cdot 59$ & 99975 & & \\
\hline
\end{tabular}




\section{INDEX}

Accidental errors, 3, 5

Airy, 131

Alternatives to Normal Error Law, 133

Amplitude of periodic terms, 171, 173

Arithmetic Mean, 9, 21

$$
\begin{array}{lll}
\text { ", } & \text { accuracy of, } 9 \\
36,37 & \text { probable error of }
\end{array}
$$

Average error, 30

Bessel, 131

Characteristic, 133

Charlier, 145

Chauvenet, 130, 131

Chauvenet's criterion, 130

Checks, 22, 80, 91

Coefficient of correlation, 154

Comparison of the two formulae for $r$, 58

Conditional equations, 124

Conditioned observations, 124

Constant error, 5, 118

Contingency, method of, 166

table, 150,154

Correction of statistics for a known probable error, 53

Correlates, 127

Correlation, 148

$$
\begin{aligned}
& \text { " coefticient, } 154 \\
& \text {, , calculation of, } \\
& 156,158 \\
& \text {, } \quad \text {, meaning of, } 169 \\
& \text {, , } \quad \text { probable error } \\
& \text {, ratio, } 160,163 \\
& \text { of, } 162 \\
& \text { " , probable error of, }
\end{aligned}
$$

Criterion, Chauvenet's, 130

" Pearson's, 141

, Peirce's, 130

Curves of error, 6

", , frequency, 18

" , presumptive error, 8

Determinant method of solution, 99

Discontinuities of periods, 208

Doolittle method of solution, 96
Eddington, vi, 15, 53, 146

Edgeworth, 145, 146

Elderton, 145, 152

Error, average, 30

" constant, 5, 118

" curve, form of the, 18

"function, tables of the, 19, 212

, law, 9

", " derived from the arithmetic mean, 22

, , Hagen's proof, 11

" " , ", generalised,

" $" 15$

, , Thomson and Tait's proof, 14

" systematic, 5, 118

Errors due to separable causes, 50

" instrumental, 2

" of observation, 1

External conditions, 2

Form of solution for normal equations, 95,110

Fourier's series for random distribu. tion, 199

, , , Turner's use of comtheorem, 179 plete, 204

Frequency curves, 6

Gauss, 9, 17, 28

Ganss's error law (see Error law).

Gläisher, 28 method of substitution, 90

Hagen's hypothesis, 11 , proof of the Error law, 11, 15

Harmonic analysis, 171 " evaluation of the coefficients, $174,181,185$

Hayford, Wright and, 128, 132

Helmert, 59

Hidden periodicities, 191

Histogram, 7

Instrument, 1

Instrumental errors, 2 
Lamb, 2

Law of Ërors, 9, 22

" , of a linenr funetion, 24

"133 " alternatives to normal,

Line of regression, 149

Lists of references, $28,128,212$

Mean, arithmetic, 9, 133

, square error, 29,33

Mensures of precision, 29

Median, 27, 133

Mode, 133

Moments, method of, 135, 136

Normal equations, 77

$, \quad, \quad \begin{gathered}\text { cheeks on forma- } \\ \text { tion, } 80 \\ \text { Examples of forma. } \\ \text { tion of, } 82\end{gathered}$
independence of, 79
solution of, 88,90
$" \quad$ error law (see Error law)
$" \quad$ place, 117

Observational equations, 76

Observations, rejeetion of, 129

Observer, 3

Pearson's differential equation, 139, 141

Peirce's criterion, 130

frequency curves, 138,142

Periodicities, hidden, 191 vestigating, 186

Periodogram, 191

Personal equation, 3

Peters' formula, 38

Precision, measures of, 29 of the probable error, 54

Presumptive errors, curve of, 8

Probability, 8

Probable error, 30

$" \quad$ " comparison of the two formulae for, 58

" , evaluation of, 31,38

" " $"$ of a linear function, 36

" , , , any function, 48,116

" ", "observational equa.

tions of unit weight, 110, 115 l'robable error of the arithmetic mean, $36 ;, 37$

" , , the unknowns, 103, $12 x$

" $\quad$ " P'cters' formula for, 3N

" " preeision of the, 5.

" , when systematic errors

are present, 51

Runks, metliod of, Itj.t

References, lists of, 28, 128, 212

Regression, curve of, 149

line of, 14!

of observations, 129

Residunl, 6

Scedastic curve, 161

Sehlomilch, 20

Schuster's Periodogram investigations, 202

Secondary analysis, 196

Separable causes, errors due to, 50

Sheppard's corrections, 140

Skewness, 134

Spearman's formulae, 164

Standard deviation, 134 "169 " probable error of,

Strattou, vi

Systematic error, 5, 118 , ,, effect on probable error, 51

Tables of $\theta(t), 19,214$

Tait, Thomson and, 14

Thiele, 145

Thomson and Tait, 14

Turner, 188, 204

Undetermined Multipliers, 127

Weight, observations of different, 61

Weighted mean, 61, 67

Weighting, methods of, 63

of observations, 60

Weights of unknowns, 105, 108

ing, 104, 113

rules for find.

Whittaker, 203

Wright and Hayford, 128, 132 


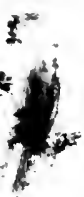






\section{University fof Toronto}

Gerstein Science Iriformation Centre

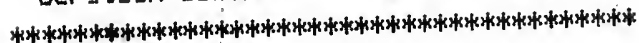

$$
01 \text { Jur, } 2000
$$

\section{MAIIE:} DANIELA MONALDI

\section{EODK =}

The theory of errors and method of least 217L1MULREDIO 


$$
x_{2}
$$

